



\title{
Multifrequenzanalyse eines Samples \\ röntgen- und optisch selektierter Aktiver Galaktischer Kerne
}

\author{
Dissertation \\ zur Erlangung des Doktorgrades \\ der Mathematisch-Naturwissenschaftlichen Fakultäten \\ der Georg-August-Universität zu Göttingen
}

\author{
vorgelegt von \\ Karsten Bischoff \\ aus Hannover
}

Göttingen 2004 
D 7

Referent:

Prof. Dr. W. Kollatschny

Korreferent:

Prof. Dr. S. Dreizler

Tag der mündlichen Prüfung: $\quad$ 15. März 2004 


\section{Inhaltsverzeichnis}

1 Einleitung \& Motivation 5

1.1 Aktive Galaxien . . . . . . . . . . . . . . . . . 5

1.2 Taxonomie zentraler AGN-Typen . . . . . . . . . . . . . . 6

1.3 Das „Unified Model“ Aktiver Galaxien . . . . . . . . . . . . . . . 7

1.3.1 Optische Spektraleigenschaften . . . . . . . . . . . . 9

1.3.2 Erscheinungsbild im Radiobereich . . . . . . . . . . . . . . . 10

1.3.3 „Broad-Line Radio Galaxies“ BLRG . . . . . . . . . . . . 11

1.3 .4 Der Staubtorus . . . . . . . . . . . . . . . . . . . 12

1.3.5 Die Broad-Line Region . . . . . . . . . . . . . . . . . . 14

1.3.6 Weitere aktuelle Beobachtungen . . . . . . . . . . . 14

1.4 Ziel der Arbeit . . . . . . . . . . . . . . . . . . . . . . . . . 15

2 Der Datensatz - Selektion, Beobachtung und Reduktion 17

2.1 „Very-Broad-Line Galaxies“ VBLG . . . . . . . . . . . . . . . . . 17

2.2 Röntgenselektierte Galaxien _. . . . . . . . . . . . . 20

2.3 Optische Beobachtungskampagnen . . . . . . . . . . . . 22

2.4 Datenreduktion . . . . . . . . . . . . . . 27

3 Optische Spektroskopie \& Klassifikation 31

3.1 Linienprofilanalyse . . . . . . . . . . . . . . . . . . 31

3.2 Klassifikation der Galaxien-Aktivität . . . . . . . . . . . . . . . 32

3.3 Häufigkeitsverteilung der AGN-Typen . . . . . . . . . . . . . . . 46

3.4 Rotverschiebung der Quellen . . . . . . . . . . . . . . . . . . . 48

3.5 Räumliche Verteilung der XGAL . . . . . . . . . . . . . . . 52 
4 Der Multifrequenzdatensatz $\mathbf{5 5}$

4.1 Korrelationsanalyse . . . . . . . . . . . . . . . . . . . . 55

4.2 Kosmologisches Modell . . . . . . . . . . . . . . . 56

4.3 Optische Helligkeiten . . . . . . . . . . . . . . . . . . 57

4.3.1 Optische Galaxienkataloge . . . . . . . . . . . . 57

4.3.2 Der optische Datensatz . . . . . . . . . . . . . . . . 58

4.4 Der Röntgenbereich . . . . . . . . . . . . . . . . . . . . . . . . . . 61

4.4.1 Röntgen-Kataloge . . . . . . . . . . . . . . 61

4.4 .2 Galaxiengruppen . . . . . . . . . . . . . . 62

4.4.3 Der Röntgen-Datensatz . . . . . . . . . . . . . . . . 63

4.5 Der Radiobereich . . . . . . . . . . . . . . . . . . 67

4.5.1 Radiodurchmusterungen . . . . . . . . . . . . . . 67

4.5.2 Quellen der Radiodaten . . . . . . . . . . . . . . . . . . . . . 69

4.5.3 Der Radio-Datensatz . . . . . . . . . . . . . . . . . . . . . 71

4.6 Der Ferninfrarotbereich . . . . . . . . . . . . . . . . . . . . . . . . . . 74

4.6.1 Ferninfrarotbeobachtungen . . . . . . . . . . . 75

4.6.2 Der FIR-Datensatz . . . . . . . . . . . . . . . 75

4.6 .3 Die Ferninfrarotfarbe . . . . . . . . . . . . . . . . . . . 79

4.7 Der Malmquist-Bias . . . . . . . . . . . . . . . . . 81

5 Flussverhältnisse und Korrelationen $\quad 85$

5.1 Verhältnis von Röntgen- zu optischem Fluss $f_{X / B} \ldots \ldots$

5.2 Die Ferninfrarot-Radio-Korrelation . . . . . . . . . . . . . . . . . . . . 90

$5.3 \mathrm{f}_{\mathrm{X} / \mathrm{FIR}}$ als Maß für Sternentstehungsaktivität . . . . . . . . . . . . 95

$5.4 \mathrm{~L}_{\mathrm{FIR}} / \mathrm{L}_{\mathrm{B}}$ als Maß der Sternentstehungsaktivität . . . . . . . . . . . . . 98

$5.5 \mathrm{f}_{\mathrm{R} / \mathrm{B}}$ als Kriterium für radiolaute und radioleise AGN . . . . . . . . . 103

5.6 Verhältnis von Röntgen- zu Radiofluss $f_{X / R}$. . . . . . . . . . . . . 107

5.7 Flussverhältnisse als Funktion der Rotverschiebung . . . . . . . . . . . 111

5.8 Diskussion der Korrelationen . . . . . . . . . . . . . . . . . . . . 113

5.8.1 Flüsse und Leuchtkräfte . . . . . . . . . . . . . . . . . . . 114

5.8 .2 Flussverhältnisse . . . . . . . . . . . . . . . 115

5.8.3 Folgerungen aus den Korrelationen . . . . . . . . . . 116 
6 Optische Spektroskopie der Broad-Line Region 119

6.1 Emissionslinienbreiten . . . . . . . . . . . . . . . . 119

6.2 Korrelationen mit der Linienbreite . . . . . . . . . . . . . . . . 127

6.2 .1 Leuchtkräfte . . . . . . . . . . . . . . . . . 127

6.2 .2 Flussverhältnisse . . . . . . . . . . . . . . . . . 130

6.3 Das Balmerdekrement . . . . . . . . . . . . . . . 132

6.4 Ergebnisse der optischen Spektroskopie . . . . . . . . . . . . 136

7 Zusammenfassung und Diskussion der Ergebnisse 143

7.1 Röntgenselektion Aktiver Galaxien . . . . . . . . . . . . . . . . . . . . 143

7.1.1 Röntgenemission Elliptischer Galaxien ． . . . . . . . . . . . 144

7.1.2 Röntgenemission Aktiver Galaxien . . . . . . . . . . . . . 145

7.1.3 Schlussfolgerungen aus der selektierten Population . . . . . . . 147

7.2 Multifrequenzdatensatz und -analyse . . . . . . . . . . . . . . . . 148

7.2.1 Der Datensatz . . . . . . . . . . . . . . . . . . . . 148

7.2.2 Flussverhältnisse . . . . . . . . . . . . . . . . . . . 149

7.3 Spektroskopie der Broad-Line Region . . . . . . . . . . . . . . 152

7.3.1 Die Emissionslinienbreite . . . . . . . . . . . . . . 153

7.3.2 Zusammenhänge mit anderen Parametern . . . . . . . . . . 153

7.3.3 Das Scheiben-Wind-Modell der BLR . . . . . . . . . . . 154

7.3.4 Das Balmerdekrement . . . . . . . . . . . . 157

7.4 Schlussfolgerungen für das „Unified Model“ . . . . . . . . . . . 158

7.5 Ausblick . . . . . . . . . . . . . . . . . . 159

8 Anhang 160

$\begin{array}{ll}\text { Abbildungsverzeichnis } & 184\end{array}$

$\begin{array}{lr}\text { Tabellenverzeichnis } & 186\end{array}$

$\begin{array}{ll}\text { Literaturverzeichnis } & 187\end{array}$ 


\section{Kapitel 1}

\section{Einleitung \& Motivation}

Diese Arbeit widmet sich dem Phänomen der Aktiven Galaxien. Dabei handelt es sich um Galaxien, die in ihren Kernen zusätzlich zu der gewöhnlichen stellaren Komponente einen Energieausstoß produzieren, der demjenigen der Summe aller Sterne in dem System gleichkommen oder ihn sogar um Größenordnungen übertreffen kann.

\subsection{Aktive Galaxien}

Aktive Galaxien sind die leuchtkräftigsten Einzelobjekte im Universum und haben daher auch eine besondere Relevanz für die Kosmologie sowie die Erforschung der Frühphasen und der Entwicklung des Universums. Auch nach mehreren Jahrzehnten ihrer Erforschung wird den Aktiven Galaxien weiterhin größtes Interesse zuteil. Nicht nur sind nach wie vor viele Detailfragen unklar, es ist bisher insbesondere auch nicht gelungen, in einem umfassenden Modell die zahlreichen Einzelaspekte konsistent zu erklären.

Um das wesentliche Merkmal Aktiver Galaxien, ihre dominierende Kernaktivität, zu betonen und gleichzeitig eine eindeutige verbale Abgrenzung z.B. zu Starburst-Galaxien zu vollziehen, wird häufig von Aktiven Galaktischen Kernen („Active Galactic Nuclei“ AGN) gesprochen. So wird auch in dieser Arbeit verfahren, ansonsten werden die Begriffe Aktive Galaxie und AGN synonym verwendet.

Allgemeines zur Historie und zu den Eigenschaften Aktiver Galaxien findet sich bei Bischoff (1993) sowie in aktuellen Übersichtsartikeln von Shields (1999), Wills (1999), Véron-Cetty \& Véron (2000) und Collin (2001).

Betrachtet man Aktive Galaktische Kerne vom Standpunkt der Beobachtung, so kann man zahlreiche verschiedene Typen unterscheiden. Andererseits ist heutzutage kaum noch strittig, dass Materieakkretion auf ein massereiches Schwarzes Loch die wesentliche Energiequelle aller AGN darstellt. Hiervon ausgehend ist der Versuch naheliegend, 
Tabelle 1.1: Vereinfachtes taxonomisches Schema nach Urry \& Padovani (1995) zur Illustration der Nomenklatur Aktiver Galaxien in Abhängigkeit von optischspektralen Eigenschaften (Emissionslinienbreite) und dem Erscheinungsbild im Radiobereich (radiolaut/radioleise). Zur besseren Übersichtlichkeit sind nur einige wenige, für diese Arbeit wichtige AGN-Typen aufgeführt.

\begin{tabular}{|c|cc|}
\hline & $\begin{array}{c}\text { Typ-2 } \\
\text { schmale Linien }\end{array}$ & $\begin{array}{c}\text { Typ-1 } \\
\text { breite Linien }\end{array}$ \\
\hline radioleise & Sy2 & $\begin{array}{c}\text { Sy1 } \\
\text { QSO }\end{array}$ \\
& $\begin{array}{c}\text { NLRG } \\
\text { radiolaut }\end{array}$ & BLRG \\
& "Edge-on“ & "Face-on“ \\
& abnehmender Winkel zur Sichtlinie \\
\hline
\end{tabular}

verschiedene Typen Aktiver Galaxien im Rahmen eines einheitlichen physikalischen Modells zu erklären. Solche Modelle werden in der Literatur als „Unified Model“ oder auch „Unified Scheme“ bezeichnet und sollen im Folgenden näher erläutert werden.

\subsection{Taxonomie zentraler AGN-Typen}

Um erste Ordnung in die Vielfalt Aktiver Galaxien zu bringen, sind einige der zentralen und auch in dieser Arbeit wichtigen Typen in Tabelle 1.1 in einem taxonomischen Schema nach Urry \& Padovani (1995) aufgetragen. Zum einen wird zwischen Typen mit schmalen (Typ-2) und breiten optischen Emissionslinien (Typ-1) unterschieden, zum anderen zwischen radioleisen und radiolauten Objekten.

Zurückgehend auf erste Arbeiten von Carl Seyfert (1943) bezeichnet man solche Systeme als Seyfert-Galaxien, die zusätzlich zur Summe der stellaren Absorptionslinienspektren Emissionslinien zeigen, die sich auch nicht durch starke Sternentstehungsausbrüche, so genannte „Starbursts“, erklären lassen. Dabei wurden zunächst rein phänomenologisch Seyfert-2 Galaxien (Sy2), die nur schmale erlaubte und verbotene Emissionslinien zeigen (Halbwertsbreite FWHM ,full width at half maximum“ ca. $500 \mathrm{~km} \mathrm{~s}^{-1}$ ), und Seyfert-1 Galaxien (Sy1) unterschieden, die zusätzlich breite erlaubte Emissionlinien aufweisen (FWHM typischerweise $2000-5000 \mathrm{~km} \mathrm{~s}^{-1}$ ). Später wurden auch 
Übergangstypen (z.B. Seyfert-1.5) beobachtet und klassifiziert, auf die in den folgenden Abschnitten noch eingegangen wird.

Der Begriff QSO leitet sich von „Quasi-stellar Object“ ab. Diese Objekte entsprechen bezüglich der spektralen Eigenschaften den Seyfert-1 Galaxien, sie sind aber weiter entfernt (und erschienen daher früher sternähnlich oder ,quasi-stellar") sowie leuchtkräftiger und müssen definitorisch abgegrenzt werden (z.B. Peterson 1988, Véron-Cetty \& Véron 2001). Gelegentlich wird synonym der Begriff „Quasar“ verwendet.

BLRG (,Broad-Line Radio Galaxies“) haben wie Seyfert-1 Galaxien und QSO breite Emissionslinien. Häufig sind diese BLRG-Linien jedoch sehr viel breiter und weisen außerdem sehr viel komplexere Linienprofile auf als typische Seyfert-1-Linien. Ferner sind diese Objekte radiolaut, d.h. sie zeigen eine starke Radioemission. Demgegenüber haben die ebenfalls radiolauten NLRG („Narrow-Line Radio Galaxies“) keine breiten optischen Emissionslinien. Auf die Untertypen FRI und FRII wird im nächsten Abschnitt 1.3.3 eingegangen.

Das Schema der Tabelle 1.1 umfasst nur fundamentale Typen; darüber hinaus gibt es noch zahlreiche weitere Typen und Untertypen, die teilweise im folgenden Abschnitt, teilweise in Kapitel 3 näher behandelt werden. Auch die bereits in diesem Schema aufgeführten Hinweise zum Blickwinkel auf die jeweiligen Objekte werden im folgenden Abschnitt anhand der Abbildung 1.1 diskutiert.

\subsection{Das „Unified Model“ Aktiver Galaxien}

Es gibt kein einheitliches, geschlossenes „Unified Model“, sondern verschiedene Autoren (z.B. Barthel 1989, Antonucci 1993, Urry \& Padovani 1995, Peterson 1997, Wills 1999) haben Ideen entwickelt und Teilaspekte zu einer zunehmend allgemeiner verbreiteten Vorstellung zusammen getragen. Insbesondere ist immer wieder versucht worden, weitere AGN-Typen zu integrieren.

Das zentrale Merkmal all dieser Vereinheitlichungsmodelle besteht darin, dass AGN nicht sphärisch symmetrisch aufgebaut sind und somit das Erscheinungsbild vom Blickwinkel abhängt. Eine der besten und übersichtlichsten Skizzen, die den möglichen Aufbau Aktiver Kerne schematisch darstellt, stammt von Wills (1999) und ist in Abbildung 1.1 wiedergegeben. Im Mittelpunkt befindet sich die zentrale Energiequelle, die in der Abbildung als kleines Scheibchen eingezeichnet ist. Es gibt kaum noch Zweifel, dass es sich hierbei um ein Materie akkretierendes Schwarzes Loch handelt und einfallende Materie eine Akkretionsscheibe um das Schwarze Loch herum bildet. Dabei kann die Ruheenergie der einfallenden Materie mit im Vergleich zur stellaren Kernfusion hoher Effizienz in Reibungs- und Strahlungsenergie umgesetzt werden. 


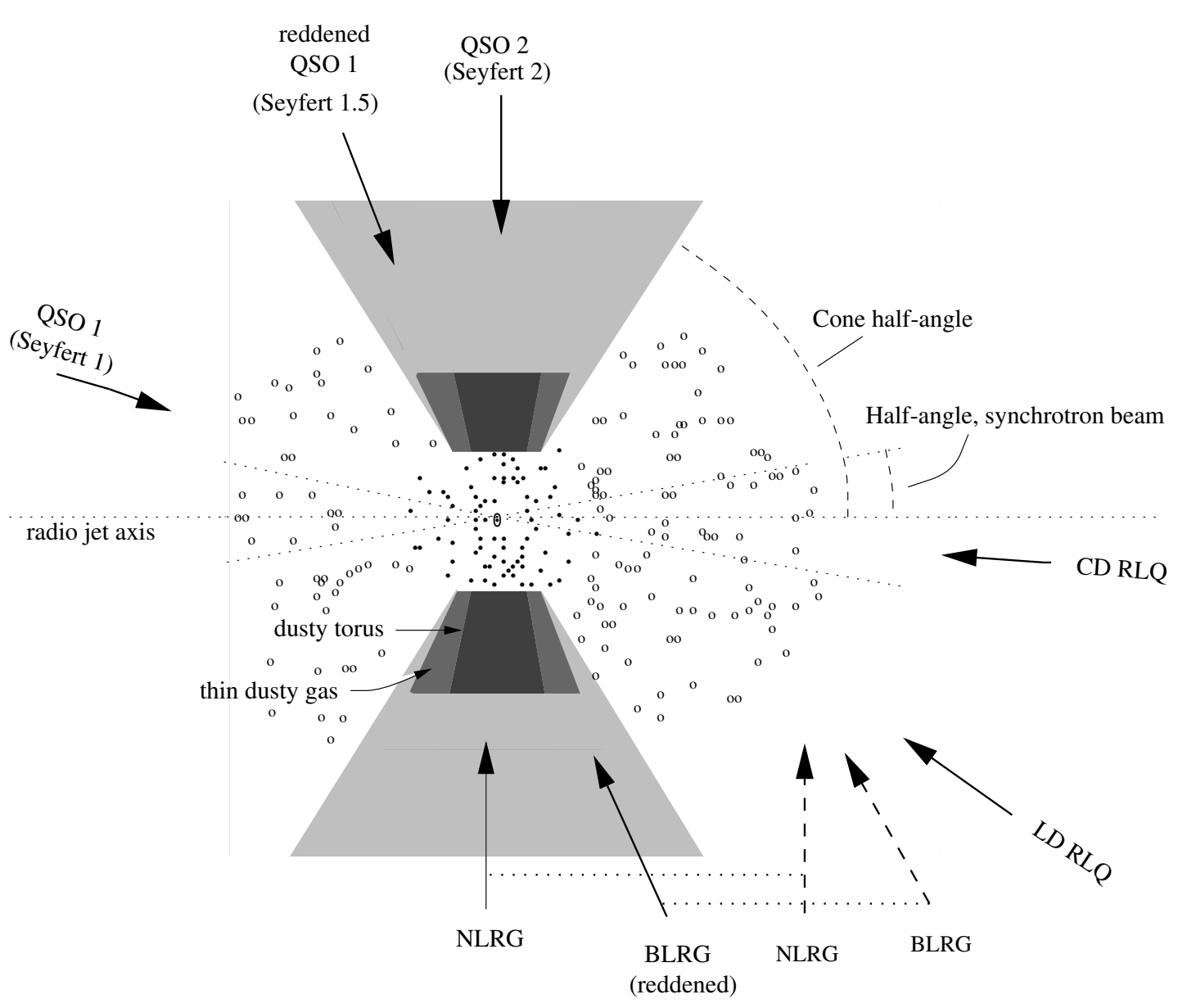

\begin{abstract}
Abbildung 1.1: Schema zur einheitlichen Darstellung Aktiver Galaxien nach Wills (1999). Unterschiedliche Blickwinkel auf die zentrale Energiequelle (Bildmitte) führen zur Beobachtung unterschiedlicher AGN-Typen. Die Broad-Line Region BLR (schwarze Punkte) kann teilweise oder komplett von absorbierendem Gas und Staub (graue und schwarze Bereiche) verdeckt sein. Zumindest Teile der Narrow-Line Region NLR (Kreise) können aus allen Richtungen gesehen werden. Oben links sind Blickrichtungen (Pfeile) auf radioleise Typen eingezeichnet, unten rechts solche auf radiolaute. Gestrichelte Pfeile zeigen Blickrichtungen auf gestreute, polarisierte BLR-Emission an. Horizontal durch das Zentrum verläuft die Achse eines eventuellen Radiojets.
\end{abstract}

Inzwischen konnten die Massen solcher Schwarzen Löcher in etlichen Fällen mit recht hoher Genauigkeit bestimmt werden (Kollatschny \& Bischoff 2002, Vestergaard 2002, Oshlack et al. 2002). Sie liegen für gewöhnliche Seyfert-1 Galaxien typischerweise im 
Bereich von $10^{7}-10^{8} \mathrm{M}_{\odot}{ }^{1}$. Die Masse des Schwarzen Lochs ist einer der wichtigsten Parameter eines AGN.

\subsubsection{Optische Spektraleigenschaften}

In unmittelbarer Umgebung (d.h. in Abständen von einigen Lichttagen bis Lichtwochen) des zentralen Schwarzen Lochs befindet sich die Materie, welche die so genannte Broad-Line Region (BLR) bildet. Sie wird von der zentralen Kontinuumsstrahlung photoionisiert und reemittiert dann ein charakteristisches Linienspektrum. Aufgrund des starken Gravitationspotenzials der Zentralmasse herrschen in diesem Bereich hohe Virialgeschwindigkeiten vor und bewirken eine kinematische Linienverbreiterung

$$
\Delta \lambda=\lambda \frac{\Delta v}{c}
$$

die üblicherweise direkt als Geschwindigkeitsdispersion $\Delta v$ angegeben wird und im Bereich zwischen ca. $1000 \mathrm{~km} \mathrm{~s}^{-1}$ bis weit über $10000 \mathrm{~km} \mathrm{~s}^{-1}$ liegen kann.

In sehr viel größeren Abständen zum Zentrum von typischerweise 100 Lichtjahren befindet sich die Narrow-Line Region (NLR). Auch hier wird die Materie durch Photoionisation zur Linienemission angeregt, die Geschwindigkeiten sind aber sehr viel geringer, so dass die Linienbreiten ca. $500 \mathrm{~km} \mathrm{~s}^{-1}$ nicht übersteigen.

Ein wesentliches Element des „Unified Models“ ist nun absorbierende Materie, die aus bestimmten Richtungen den direkten Blick auf die Broad-Line Region verhindert, wohingegen die sehr viel ausgedehntere Narrow-Line Region auch aus diesen Blickrichtungen frei sichtbar ist. Die absorbierende Materie wird häufig als Staubtorus oder Staubring beschrieben, was ursprünglich auf eine Arbeit von Antonucci (1982) zurückgeht, und ist in der Abbildung 1.1 durch die grauen und schwarzen Zonen ober- und unterhalb der zentralen Quelle skizziert.

Blicken wir nun von der Seite auf das Objekt der Abbildung 1.1, sehen wir die BLR und mithin auch breite Emissionslinien - wir beobachten eine Typ-1 Quelle (z.B. Seyfert-1, QSO, aber auch BLRG). Von oben oder unten könnte man jedoch die BLR nicht sehen und beobachtete dann ein Typ-2 Objekt (z.B. Seyfert-2). Wie schon in der Tabelle 1.1 aufgeführt nennt man diese beiden Extremfälle auch ,face-on“ (Aufsicht) und ,edge-on“ (Kantensicht) Orientierung. Den Winkel zwischen der Scheiben- und der Himmelebene bezeichnet man als Inklination - die exakte Aufsicht entspricht somit einem Inklinationswinkel von $0^{\circ}$, die Kantensicht entspricht $90^{\circ}$.

\footnotetext{
${ }^{1}$ Sonnenmasse $1 \mathrm{M}_{\odot}=1.989 \cdot 10^{30} \mathrm{~kg}$
} 
Es werden ferner Übergangstypen wie z.B. Seyfert-1.5 Galaxien beobachtet, bei denen die erlaubten Emissionlinien sowohl schmale als auch breite Komponenten vergleichbarer Stärke zeigen (Osterbrock 1977, 1989). Dieser Typ ist im Schema der Abbildung 1.1 mit einem teilweise absorbierten Sehstrahl eingezeichnet.

QSO mit geröteten Spektren (,reddended QSO 1“) können noch besser mit solch einem teilabsorbierten Sehstrahl assoziiert werden, da die Rötung als direkter Effekt der partiellen Absorption durch Staub verstanden werden kann. Teilabsorption einhergehend mit Rötung ist zumindest auch für einen Teil der BLRG Population (s.u.) möglich, was ebenfalls durch einen Sehstrahl skizziert wird.

Auf diese Weise lassen sich die verschiedenen Emissionslinienbreiten im optischen Spektrum unmittelbar über einen Orientierungseffekt erklären. Sehr starke Unterstützung erfuhr dieses Modell durch die erste Beobachtung einer so genannten versteckten Broad-Line Region (hidden BLR bzw. HBLR) im polarisierten Licht der prototypischen Seyfert-2 Galaxie NGC 1068 durch Miller \& Antonucci (1983). Diese Beobachtung wurde sogleich damit erklärt, dass die BLR aufgrund der Typ-2 Orientierung des Objektes zwar nicht direkt sichtbar ist, das von ihr ausgehende Licht aber an Staubteilchen außerhalb des abschattenden Materials gestreut wird und so zum Beobachter gelangt.

Da uns in diesem Fall also nur gestreutes Licht aus der BLR erreicht, ist es einerseits polarisiert und macht andererseits typischerweise nur etwa $1 \%$ des uns direkt erreichenden Lichts aus der NLR aus, d.h. der Nachweis ist schwierig. Darum sind bis heute nur von einer relativ geringen Zahl von Typ-2 Galaxien solcherlei Beobachtungsdaten verfügbar (s.u.).

\subsubsection{Erscheinungsbild im Radiobereich}

Analog zum Optischen lassen sich auch die stark unterschiedlichen Erscheinungsformen im Radiobereich deuten. Grundlage dafür sind die Konzepte von Blandford \& Rees (1974), die erstmals Radio-Jets und -Beams (stark fokussierte, relativistische Plasmastrahlen) beschrieben und somit erkannten, dass hier hochgradig nichtsphärische Strukturen vorliegen und daher die Blickrichtung von größter Bedeutung ist.

Ein Vereinheitlichungsschema für die verschiedenen radiolauten Objekte wurde erstmals von Barthel (1989) veröffentlicht. Der Effekt des sog. „Doppler-Boosting“, einer hohen Verstärkung der Strahlung durch anisotrope Emission beim axialen Aufblick auf einen relativistischen Jet, war hierbei ebenso wichtig wie wiederum die nicht sphärischsymmetrische Absorption und Abschattung des Zentrums. Der Grundgedanke war aber auch bereits in diesem Modell, dass ein AGN die zentrale Energiequelle sei - ein damals noch recht revolutionärer Gedanke, da der AGN selbst in der bis dato bekannten Form nicht sichtbar war. 
In Abbildung 1.1 ist die Achse des Radio-Jets horizontal eingezeichnet, also parallel zur Symmetrieachse des Staubtorus. Diese Annahme ist plausibel, aber nicht unbedingt zwingend, sie wird jedoch inzwischen auch durch Beobachtungen unterstützt, z.B. durch den sog. Ausrichtungs-Effekt (,alignment effect"), den Chambers et al. (1987) und Mc Carthy et al. (1987) bei leuchtkräftigen, höher rotverschobenen Radiogalaxien fanden.

Radiogalaxien mitsamt ihren Jets sind die größten Einzelobjekte im Universum. Bereits einer der ersten beobachteten Jets in der klassischen Radiogalaxie Cygnus A, einer im Optischen sehr unscheinbaren Galaxie, hat eine Ausdehnung von etwa 200 kpc und übertrifft somit die gesamte Galaxie deutlich, während die Gesamtausdehnung der Radiojets der Galaxie 3C 236 immense 5.6 Mpc beträgt (Perley et al. 1984). Somit können diese Strukturen zwar keinesfalls verdeckt werden, durch die genannten anisotropen relativistischen Effekte spielt aber dennoch der Blickwinkel auf die Radiostrukturen eine entscheidende Rolle.

In der Abbildung 1.1 bezeichnen CD RLQ Kern-dominierte radiolaute Quellen und LD RLQ die entsprechenden Lobe-dominierten Gegenstücke, wobei man als Lobes die Radiokeulen, das heißt sehr auffällige, radiohelle Endbereiche der Jets bezeichnet.

$\mathrm{Zu}$ den Kern-dominierten Quellen gehören auch die recht seltenen BL Lac Objekte (oder kurz BLL), die nach der ursprünglich als Veränderlicher (Stern) eingestuften Quelle BL Lacertae im Sternbild Eidechse benannt sind. Ihre optischen Spektren zeigen keinerlei Linien (bzw. in Einzelfällen höchstens extrem schwache Linien), sondern nur ein strukturloses Kontinuum. Vermutlich sehen wir bei diesen Objekten direkt auf den zufällig auf uns zu gerichteten Jet und messen damit hochgradig verstärkte Jetemission, die die unterliegende Galaxie völlig überstrahlt (Kotilainen et al. 1998, Urry et al. 1999).

Lange Zeit war die Verbindung von Jets und Akkretionsscheiben nur theoretischer Natur, insbesondere weil direkte Beobachtungen der Scheiben unmöglich waren. Hochaufgelöste Bilder des Hubble Weltraumteleskops von so genannten Herbig-Haro Objekten, jungen Sternen in ihrer Entstehungsphase, zeigen direkt Jets senkrecht zu zirkumstellaren Scheiben und unterstützen die theoretischen Vorstellungen nahezu perfekt. Als Beispiel ist in Abbildung 1.2 ein HST-Bild von HH-30 zu sehen (Burrows et al. 1996). Zwar ist die Physik der galaktischen, hochrelativistischen Jets ungleich komplexer als die der stellaren Jets in Herbig-Haro Objekten, aber dennoch entsprechen diese hervorragend den prinzipiellen Ideen und Vorstellungen.

\subsection{3 „Broad-Line Radio Galaxies“ BLRG}

BLRG („Broad-Line Radio Galaxies“) definieren sich sowohl über ihre Radio- wie auch über ihre optischen Spektraleigenschaften, indem sie - wie schon der Name sagt - breite bzw. sehr breite optische Emissionslinien mit großer Radiohelligkeit verbinden. Da sie 


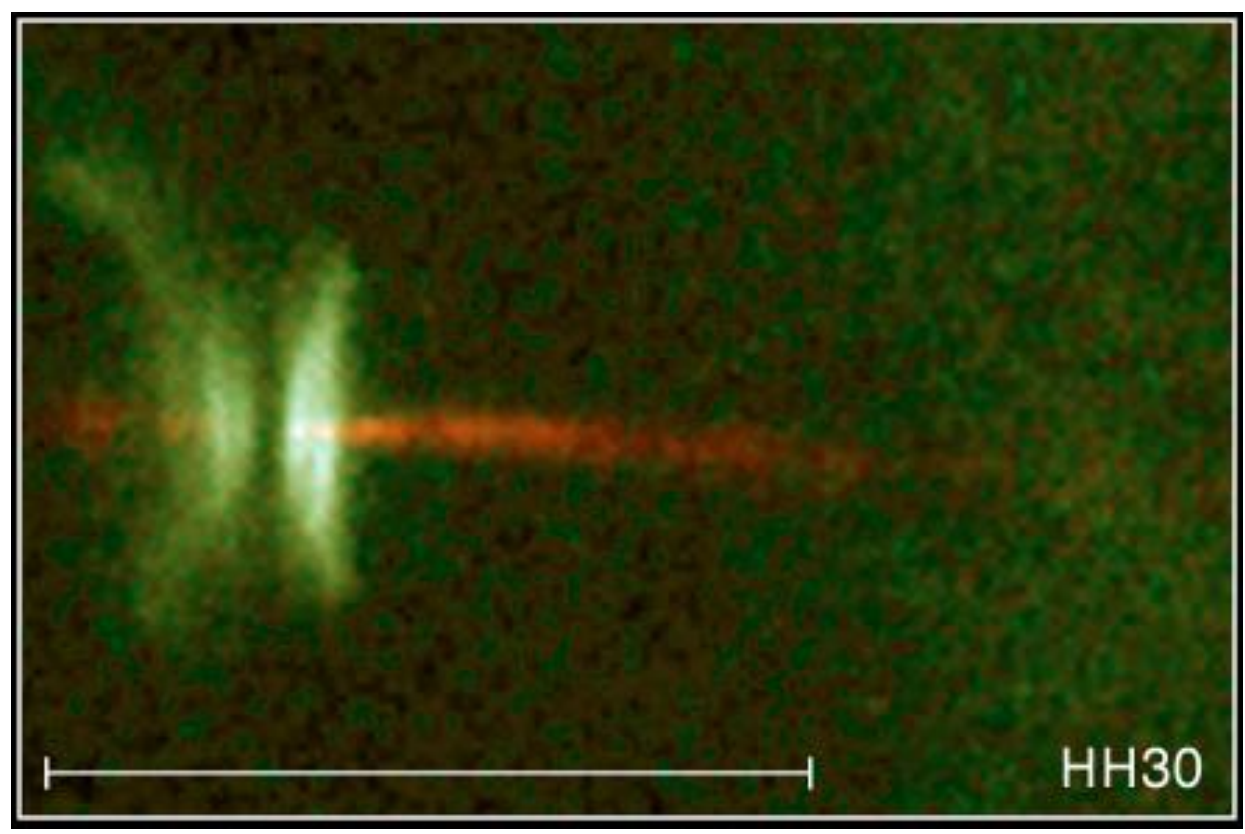

Abbildung 1.2: HST-Bild des Herbig-Haro Objektes HH-30 (Burrows et al. 1996). Obwohl es sich um einen jungen Stern in seiner Entstehungsphase handelt, entspricht die hier vertikal orientierte zentrale dunkle Scheibe, die den eigentlichen Protostern verdeckt, mit einem senkrecht dazu entweichenden Jet nahezu exakt dem Bild, das das „Unified Model“ auch von einem AGN zeichnet. Die in der Abbildung angegebene Skala entspricht $1000 \mathrm{AE}$, also dem tausendfachen Abstand Sonne - Erde $\left(1 \mathrm{AE}=1.496 \times 10^{11} \mathrm{~m}\right)$.

von besonderer Bedeutung in dieser Arbeit sind, werden sie im folgenden Kapitel noch ausführlicher behandelt (s. Abschnitt 2.1).

Auch Sichtlinien zu BLRG sind in Abbildung 1.1 eingezeichnet, ebenso wie zu ihren Gegenstücken mit schmalen optischen Emissionslinien, den NLRG (,Narrow-Line Radio Galaxies"). Die Markierung mittels jeweils zweier Sichtlinien deutet bereits an, dass es möglicherweise zwei Populationen oder - im Rahmen des Modells genauer gesagt zwei unterschiedliche Möglichkeiten der Entstehung gleich klassifizierter Objekte gibt.

Der Begriff NLRG ist weniger gebräuchlich. Wie schon im Schema der Tabelle 1.1 angedeutet ist, zählen die geläufigeren Typen Fanaroff-Riley (FR) I und II Galaxien zu den NLRG. Es handelt sich dabei um eine Klassifikation von Radiogalaxien, die auf eine Arbeit von Fanaroff \& Riley (1974) zurückgeht. FRI-Galaxien haben die höchste Radioleuchtkraft in der Nähe des Zentrums, während FRII-Galaxien über so genannte „Hotspots“ verfügen, die für eine hohe Leuchtkraft in den äußeren Bereichen der beiden Radiokeulen verantwortlich sind. 


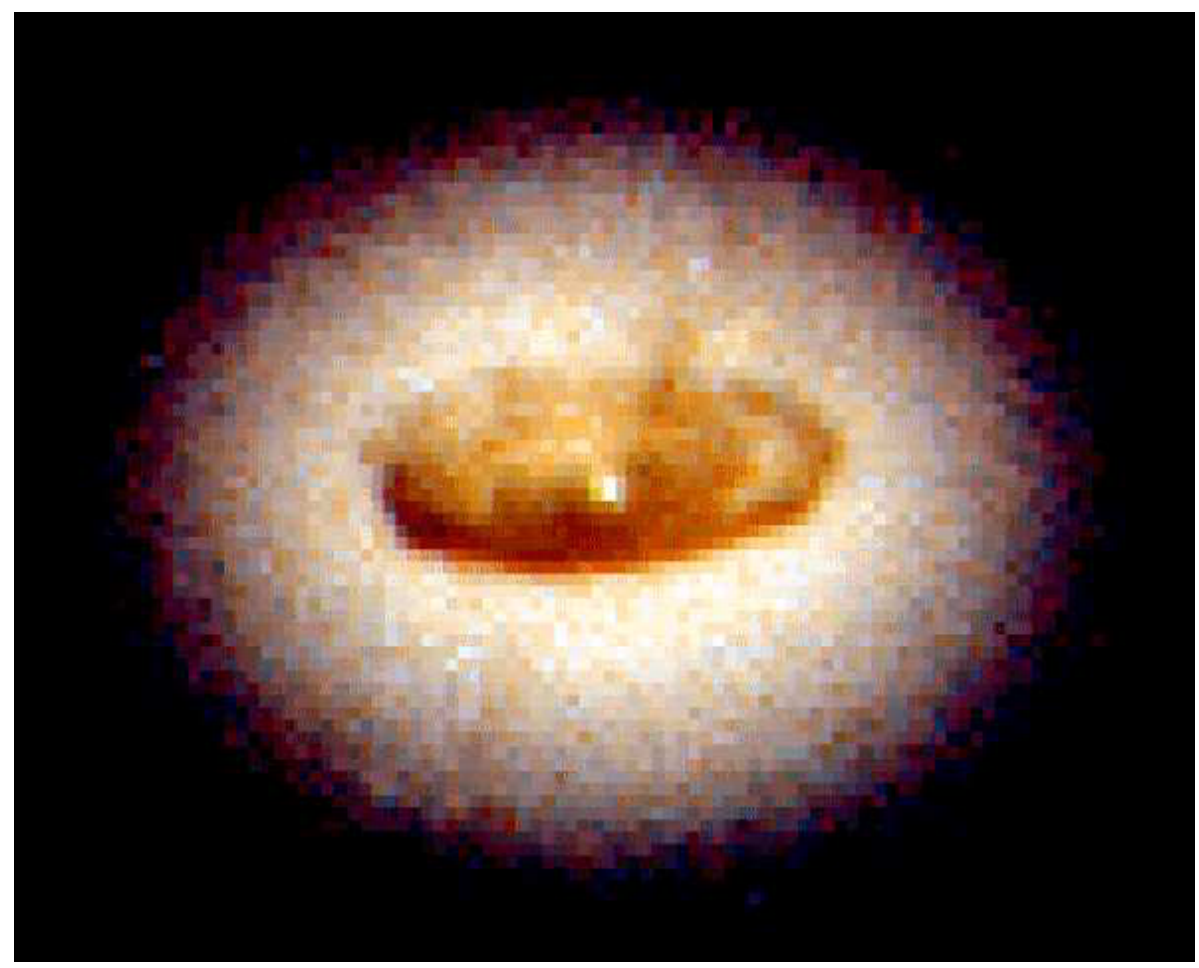

Abbildung 1.3: HST-Bild des Zentralbereichs der Radiogalaxie NGC 4261 (Jaffe et al. 1996). Ein abschattender, dunkler Torus um das Zentrum ist direkt zu sehen.

\subsubsection{Der Staubtorus}

Wie bereits erwähnt ist ein abschattender Staubtorus bzw. eine dicke Scheibe absorbierenden Materials ein entscheidendes Element des „Unified Models“. Es gibt inzwischen auch durch mehrere direkte Beobachtungen starke Evidenz für die Existenz eines solchen zentralen Staubtorus.

Eine der ersten Beobachtungen dieser Art gelang mit dem Hubble Weltraumteleskop (HST) im Zentrum in der Radiogalaxie NGC 4261 und ist in Abbildung $1.3 \mathrm{zu}$ sehen (Jaffe et al. 1996). Obschon die Interpretation der Daten nicht unumstritten war, konnten die Autoren in einer detaillierteren Untersuchung mit Hilfe von HST-Spektren die Masse des zentralen Schwarzen Loches zu etwa $5 \cdot 10^{8} M_{\odot}$ abschätzen. Ferner bestimmten sie den Positionswinkel der Hauptachse der Staubscheibe zu $163^{\circ} \pm 2^{\circ}$, denjenigen der Galaxie zu $158^{\circ} .5 \pm 0^{\circ} .4$ und den des Radiojets zu $88^{\circ} \pm 1^{\circ}$ (Birkinshaw \& Davies 1985), d.h. die drei Achsen sind nur grob aneinander ausgerichtet. Weitere Untersuchungen im Radiobereich per VLBI (,,Very Long Baseline Interferometry“, eine räumlich sehr hochauflösende interferometrische Beobachtungstechnik) sowie eine Analyse des Radiojets auf verschiedenen räumlichen Skalen wurden von Jones et al. (2000) durchgeführt. 
Eine der jüngsten direkten Beobachtungen stammt von Klöckner et al. (2003), die mit Radio-VLBI Messungen einen rotierenden Staubtorus aus molekularem Gas um das Zentralobjekt der Seyfert-Galaxie Mkn 231 gefunden haben. Dieser Torus, dessen Hydroxyl $(\mathrm{OH})$-Emission die Autoren gemessen haben, befindet sich in einem Abstand zwischen 30 und 200 pc vom Zentrum dieser leuchtkräftigsten Infrarotgalaxie im lokalen Universum.

In einer neuen theoretischen Arbeit diskutieren Vollmer et al. (2004) verschiedene Geometrien des Torus bzw. der Scheibe und machen Abschätzungen über die Akkretionsrate. Sie finden ferner, dass ein Kollisionsmodell bestehend aus quasi-stabilen, selbstgravitierenden Wolken die aktuellen Beobachtungen am besten erklärt.

\subsubsection{Die Broad-Line Region}

Diese Beobachtungen sagen noch nichts über die Natur bzw. die Herkunft des Staubtorus aus. Durch eine detaillierte Linienprofilanalyse von zeitlich und spektral hochaufgelösten Spektren der Seyfert-1 Galaxie Mrk 110, die in einer mehrwöchigen Variabilitätskampagne mit dem 9m Hobby-Eberly Teleskop (HET) aufgenommen wurden, haben wir starke Evidenz für eine scheibenförmige Broad-Line Region (BLR) in dieser Galaxie gefunden (Kollatschny et al. 2001, Kollatschny \& Bischoff 2002).

Unsere Beobachtungen entsprechen nahezu perfekt dem Scheiben-Wind-Modell von Königl \& Kartje (1994) und Kartje et al. (1997). Dabei bilden die äußeren, kühlen Bereiche der Akkretionsscheibe den abschattenden Torus, und es ist somit keine zusätzliche hypothetische Komponente nötig. Die Dicke der kühlen Scheibe entspricht gut den Anforderungen des „Unified Models“, die BLR wird von den Gaswolken gebildet, die im von der Scheibe abströmenden Wind mitgetrieben werden. Dieses Modell wird in Kapitel 7 noch weiter diskutiert werden.

Mit unseren Beobachtungen haben wir so erstmals ein genaues Bild der Zentralregion von Mrk 110 erhalten. Die Masse des Schwarzen Lochs konnten wir auch mit Hilfe der gemessenen Gravitationsrotverschiebung zu $1.4 \pm 0.3 \cdot 10^{8} \mathrm{M}_{\odot}$ bestimmen (Kollatschny 2003). Die genaue Größenskala haben wir durch die Beobachtung verschiedener Emissionslinien (Balmerlinien, He I und He II) ausgemessen.

\subsubsection{Weitere aktuelle Beobachtungen}

An dieser Stelle seien noch exemplarisch zwei weitere aktuelle Beobachtungen erwähnt, die gut zeigen, dass unser Bild Aktiver Kerne ständig komplettiert und konkretisiert wird. 


\section{Infrarot-Beobachtungen mit ISO}

Weitere Evidenz für das „Unified Model“ haben neue Beobachtungen mit dem InfrarotObservatorium ISO ergeben. Meisenheimer et al. (2001) untersuchten zehn Paare aus leuchtkräftigen Radiogalaxien und Quasaren mit dem Spektrometer ISOPHOT im Wellenlängenbereich von 5 bis $180 \mu \mathrm{m}$. Beim Vergleich der Radiogalaxien und Quasare konnten sie im beobachteten Wellenlängenbereich keine Unterschiede ausmachen. Die Autoren schließen daraus, dass beide Arten Aktiver Galaxien einen gleichartigen Kern haben und sehen ihre Beobachtungen als starke Bestätigung für die Vereinheitlichungstheorie.

\section{Spektropolarimetrie der HBLR}

Neue spektropolarimetrische Beobachtungen von etwa 50 Seyfert-2 Galaxien über sieben Jahre durch Tran $(2001,2003)$ lieferten Hinweise, die den bisher geschilderten Vorstellungen des „Unified Models“ partiell widersprechen. Bei einem Teil der Quellen (etwa der Hälfte) fand Tran im polarisierten Licht eine versteckte Broad-Line Region (hidden Broad-Line Region HBLR), bei einem anderen Teil fand er eine solche nicht.

Während dies früheren Beobachtungen entspricht, fand Tran weiterhin, dass die HBLRSeyfert-2 Galaxien ein höheres Radio/FIR-Emissionsverhältnis, wärmere Staubtemperaturen und letztendlich leuchtkräftigere Kerne enthalten als Seyfert-2 Galaxien ohne HBLR. Der Grad der Abschattung der BLR bzgl. unserer Sichtlinie scheint hingegen bei allen seinen Quellen ähnlich zu sein. Daher glaubt Tran (2003), zwei intrinsisch unterschiedliche Populationen von Seyfert-2 Galaxien beobachtet zu haben und bewegt sich somit außerhalb des gängigen „Unified Models“.

Den Effekt, dass HBLR-Seyfert-2 Galaxien wärmere Staubtemperaturen zeigen als Seyfert-2 ohne HBLR, hatten bereits Heisler et al. (1997) anhand eines Samples von 16 Quellen beobachtet. Sie deuteten diesen Effekt allerdings als Bestätigung des „Unified Models" und vermuteten, dass im ersten Fall die Sichtlinie nahe am Rande des Staubtorus verläuft, während bei letzteren nicht nur die BLR, sondern auch der wärmere Staub vom Torus verdeckt wird und somit von uns aus nicht beobachtbar ist.

\subsection{Ziel der Arbeit}

Der im vorherigen Abschnitt besprochene aktuelle Kenntnisstand der Natur Aktiver Galaxien sowie die geschilderten Beobachtungen zeigen unmittelbar, dass alle zur Verfügung stehenden Techniken genutzt werden müssen, um unser Bild Aktiver Galaxien weiter zu entwickeln. 
Ziel dieser Arbeit ist es, das Phänomen Aktiver Galaktischer Kerne im Hinblick auf das „Unified Model“ insbesondere unter drei Hauptgesichtspunkten zu untersuchen:

- es sollen spezielle, bisher wenig untersuchte Typen Aktiver Galaxien analysiert werden: zum einen VBLG (,Very-Broad-Line Galaxies“), die aufgrund ihrer breiten, außergewöhnlichen optischen Linienprofile selektiert wurden (s. Abschnitt 2.1), zum anderen röntgenselektierte, ,neue“ AGN (s. Abschnitt 2.2);

- durch eine Multifrequenzanalyse sollen die vier Frequenzbänder Röntgen, Optisch, Radio und FIR parallel analysiert werden, um ein umfassendes Gesamtbild über die Emissionen aus verschiedenen Bereichen der Galaxien zu erhalten (s. Kapitel 4);

- die Broad-Line Region soll mit Hilfe optischer Spektroskopie detailliert untersucht werden. Dabei kommt der Analyse der Breite der erlaubten Emissionslinien besondere Bedeutung zu, da die Linienbreite einen entscheidenden Parameter der Broad-Line Region darstellt und insbesondere in Modellen mit scheibenförmiger Geometrie in direktem Zusammenhang mit dem Blickwinkel auf den Aktiven Kern stehen könnte (s. Kapitel 6). 


\section{Kapitel 2}

\section{Der Datensatz - Selektion, Beobachtung und Reduktion}

Um das Phänomen Aktiver Galaktischer Kerne insbesondere hinsichtlich des im vorherigen Kapitel beschriebenen „Unified Models“ zu untersuchen, haben wir uns zu einer gemeinsamen und umfassenden Multifrequenzanalyse zweier sehr spezieller Arten Aktiver Galaxien entschlossen: zum einen der VBLG („Very-Broad-Line Galaxies“), die sich durch außergewöhnliche Linienprofile im optischen Spektrum stark von anderen AGN abheben, und zum anderen der röntgenselektierten AGN, einer Klasse neu entdeckter AGN, die erst durch eine umfangreiche Auswertung der Himmelsdurchmusterung des Röntgensatelliten ROSAT gefunden werden konnten.

Diese beiden Untergruppen bilden gemeinsam das dieser Arbeit zugrunde liegende Sample und werden in den zwei folgenden Abschnitten genauer definiert und beschrieben.

\section{1 „Very-Broad-Line Galaxies“ VBLG}

Eine seltene und besondere Klasse Aktiver Galaxien sind die so genannten BLRG (,Broad-Line Radio Galaxies“). Da sie breite Emissionslinien zeigen, gehören sie zu den Typ-1 AGN und sind formal betrachtet eine spezielle Ausprägung der Seyfert-1 Galaxien. Sie haben allerdings i.a. wesentlich breitere Linienprofile als letztere und können im Extremfall Linienbreiten von bis zu $40000 \mathrm{~km} \mathrm{~s}^{-1}$ FWZI (,full width at zero intensity") erreichen (entsprechend einigen $100 \AA \AA$ im Optischen je nach Linie und Rotverschiebung). Außerdem weisen sie häufig ungewöhnliche und pekuliare Profilformen auf. Prototypische, schon relativ früh genauer untersuchte BLRG sind Arp 102B, 3C 332 und 3C 390.3. 
Das Linienprofil der breiten H $\alpha$-Emission von Arp 102B zeigt eine sog. Doppel-Peak Struktur (s. Abbildung 2.1), die weiter unten noch ausführlicher diskutiert wird. Dieses kann keinesfalls durch ein einfaches Geschwindigkeitsfeld in der Broad-Line Region erklärt werden, insbesondere auch nicht durch radiale Bewegungen. Chen, Halpern \& Filippenko (1989) haben gezeigt, dass der Doppel-Peak jedoch durch die Emission einer Akkretionsscheibe verursacht sein könnte. Das spezielle Modell von Chen, Halpern \& Filippenko (1989) konnten wiederum Miller \& Peterson (1990) aufgrund des Variationsverhaltens der postulierten Akkretionsscheibenemission widerlegen, womit jedoch nicht die prinzipielle Deutung dieser Profilform durch Akkretionsscheibenemission ausgeschlossen wird.

Die Galaxie 3C 332 zeigt ein wesentlich breiteres H $\alpha$-Profil (FWZI $\approx 30000 \mathrm{~km} \mathrm{~s}^{-1}$ ), ebenfalls mit einem Doppel-Peak. Auch diese Emission versuchte Halpern (1990) mit Hilfe einer Akkretionsscheibe zu erklären. Eine Analyse der Profilvariationen der Galaxie 3C 390.3 wurde erstmals von Veilleux \& Zheng (1991) durchgeführt, später war dieses Objekt auch Gegenstand einer großen internationalen Variabilitätskampagne (Dietrich et al. 1998).

Es gibt keine allgemeingültige, etablierte Definition für BLRG. Sie wurden im Rahmen von ersten Radiobeobachtungen als Radioquellen eingeführt (z.B. Matthew et al. (1964)). Angefangen mit einer Arbeit von Sandage (1973) wurden ihre optischen Spektren seither oft als stark gerötet beschrieben. Besonderes Interesse kommt den BLRG im Rahmen des „Unified Models“ zu, weil sie darin einen Grenzfall darstellen könnten, bei dem wir zwar einerseits die Broad-Line Region direkt einsehen, die Sichtlinie aber andererseits so nahe am abschattenden Staubtorus verläuft, dass die Zentralquelle teilweise verdeckt wird (Barthel 1989, Urry \& Padovani 1995).

Cohen et al. (1999) beschrieben die Spektren etlicher BLRG als Überlagerung eines geröteten Quasar-Spektrums und polarisierter, reflektierter Quasar-Emission. Auch Dennett-Thorpe et al. (2000) fanden eine Population mit stark geröteten Spektren, während van Bemmel \& Barthel (2001) hingegen zu dem Ergebnis kamen, dass BLRG zumindest auf größeren Skalen relativ staubarm sind. Sie propagierten die These, dass es sich um alte AGN handele.

Über die Eigenschaften im Radiobereich und inbesondere über die Orientierung der Radioachse in BLRG wird seit langem kontrovers diskutiert. In einer neueren Untersuchung von zwölf BLRG aus dem 3C-Radiokatalog fanden Dennett-Thorpe et al. (2000) zwei unterschiedliche Populationen: zum einen QSO ähnliche Objekte mit geringerer Leuchtkraft aber vergleichbarer Orientierung, d.h. einer Radioachse mit geringem Öffnungswinkel zur Sichtlinie, zum anderen entferntere, leuchtkräftigere Quellen mit größerem Winkel zur Sichtlinie. Evidenz für den ersten Fall hatte es auch schon aus Beobachtungen scheinbarer Überlichtgeschwindigkeiten, so genannter ,superluminal motions“, in BLRG gegeben (Alef et al. 1996). 


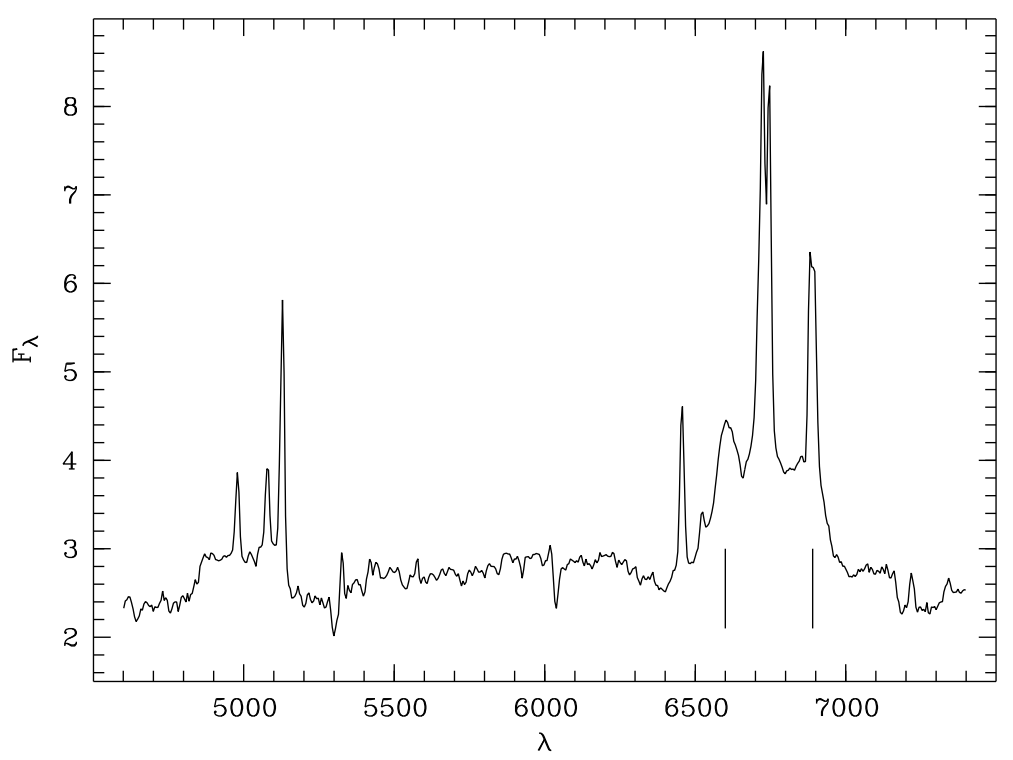

\begin{abstract}
Abbildung 2.1: Optisches Spektrum der prototypischen BLRG Arp 102B. Die komplexe und ungewöhnliche Profilstruktur ist offensichtlich. Im H $\alpha$-Profil ist die „double peaked“" (DP) Form leicht zu erkennen: das linke Maximum ist bei $6600 \AA$ gut sichtbar, das rechte bei $6900 \AA$ ist jedoch von den [S II] 66717,6731 -Linien überlagert (s. Markierungen). Im H $\beta$-Profil sind die entsprechenden Strukturen weniger stark als Schultern ausgeprägt. Man sieht weiterhin, dass die breiten Linienkomponenten im Vergleich zu den schmalen und auch zur Kontinuumsemission relativ schwach sind - dieses wird bei BLRG häufig beobachtet.
\end{abstract}

Zu den auffälligsten Merkmalen der BLRG gehören zweifelsohne die überdurchschnittlich breiten Emissionslinien mit häufig sehr ungewöhnlichen und pekuliaren Linienprofilen. Unter diesen wiederum wurden die so genannten „,double peaked“ (DP) Profile intensiv diskutiert. Im Gegensatz zu üblichen Linienprofilen habe diese nicht ein zentrales Maximum, sondern zwei Maxima links und rechts des Linienzentrums. Das bezieht sich auf das breite Linienprofil; in der Regel ist eine schmale Linienkomponente überlagert und erzeugt so im Gesamtprofil zusätzlich ein zentrales Maximum. Sind die dezentralen Maxima schwach ausgeprägt, können sie Schultern ähneln und werden dann als „twin shoulders" bezeichnet. Insgesamt können sich so sehr unregelmäßige Linienprofile ergeben.

Als prototypisches Beispiel aus unserem Sample, das viele der hier vorgestellten Charakteristika zeigt, ist in Abbildung 2.1 ein Spektrum der DP-BLRG Arp 102B zu sehen.

Sehr ähnliche DP-Linienprofile zeigen auch bestimmte Doppelsterne, die so genannten kataklysmischen Veränderlichen (cataclysmic variables CV). Bei diesen ist die Emission einer rotierenden Akkretionsscheibe schon länger als Ursache für das DP-Profil 
bekannt (z.B. Young \& Schneider (1980), Marsh (1988)). Daher lag eine entsprechende Erklärung für die BLRG nahe und wird nach wie vor am häufigsten präferiert. Eracleous \& Halpern $(1994,2003)$ fanden, dass $20 \%$ aller BLRG DP-Profile zeigen. Von diesen wiederum konnten sie 60\% mit einem einfachen Modell einer rotierenden, relativistischen Akkretionsscheibe erklären. Es werden aber auch nach wie vor alternative Ideen wie bipolare Ausströmungen oder eine anisotrop bestrahlte Broad-Line Region diskutiert.

Eine der interessantesten alternativen Erklärungen sieht ein binäres Schwarzes Loch und damit auch eine doppelte Broad-Line Region als Ursache der DP-Profile. Diese Idee geht auf die Analyse von Spektren der klassischen BLRG 3C390.3 durch Gaskell (1996) zurück. Unterstützung erhielt diese Vorstellung jüngst durch VLBI-Beobachtungen der Radiogalaxie 3C66B, die von den Autoren als direkte Detektion eines supermassiven doppelten Schwarzen Lochs proklamiert wurden (Sudou et al. 2003). Den Zusammenhang zwischen den beschriebenen Eigenschaften im optischen und im Radiobereich zu erklären ist eine der entscheidenden Herausforderungen an ein allgemeingültiges „Unified Model“.

Eracleous \& Halpern (1994, 2003) verwenden für die von ihnen untersuchten Objekte nicht den Begriff BLRG, sondern DP-Emissionslinien AGN. Diese Diktion wird auch von Strateva et al. (2003) benutzt, die ein Sample entsprechender Quellen auf Basis des SLOAN Digital Sky Surveys SDSS ${ }^{1}$ (York et al. 2000, Stoughton et al. 2002, Abazajian et al. 2003) zusammengestellt haben.

Für diese Arbeit haben wir ein Sample von Objekten zusammengestellt, die entweder besonders breite (FWHM $>5000 \mathrm{~km} \mathrm{~s}^{-1}$ ) oder besonders ungewöhnliche (asymmetrische, pekuliare, DP) optische Linienprofile zeigen und sich damit eindeutig von der großen Mehrheit gewöhnlicher Seyfert-1 Galaxien abheben. Andere Eigenschaften, wie z.B. die Radioemission, waren keine Selektionskriterien. Ein Teil der Objekte dieses Samples wird in der Literatur als BLRG, ein anderer Teil als DP-AGN bezeichnet. Etliche werden auch einfach als AGN oder Seyfert-Galaxien geführt. Aufgrund der Selektionskriterien und der oben geschilderten Probleme mit der Nomenklatur werden sie im Folgenden gemäß ihrer dominierenden Eigenschaft als VBLG (,Very-Broad-Line Galaxies") bezeichnet.

VBLG gehören zweifelsohne zu der übergeordneten Gruppe der Typ-1 AGN. Der Übergang zwischen gewöhnlichen Seyfert-1 Galaxien und VBLG ist fließend; es gibt kein scharfes Kriterium, das eine eindeutige Abgrenzung erlaubte.

\footnotetext{
${ }^{1}$ siehe http://www.sdss.org/
} 


\subsection{Röntgenselektierte Galaxien}

Aktive Galaktische Kerne sind als starke Röntgenquellen bekannt. Eine frühe systematische Untersuchung dazu wurde von Fabbiano et al. (1992) anhand des „X-ray Cata$\log$ and Atlas of Galaxies" durchgeführt. Dieser Katalog beruht auf Beobachtungen des Röntgen-Satelliten EINSTEIN und enthält 493 Galaxien. Es ergaben sich typische Röntgenleuchtkräfte im Bereich von $\mathrm{L}_{X}=10^{38}-10^{42} \mathrm{erg} \mathrm{s}^{-1}$ für normale Galaxien und von $\mathrm{L}_{\mathrm{X}}=10^{42}-10^{44} \mathrm{erg} \mathrm{s}^{-1}$ für AGN.

Die erste Himmelsdurchmusterung im weichen Röntgenbereich (0.1-2.4 keV) wurde mit dem ROSAT-Satelliten durchgeführt (Trümper 1983). Basierend auf dieser ,ROSAT All-Sky Survey“ (RASS) genannten Durchmusterung (Voges 1993) wurde der „RASS bright source catalog“ (1XRS/RASS-BSC) (Voges et al. 1996, 1999) erstellt, der 18811 Quellen enthält und somit einen immensen Fortschritt gegenüber dem o.g. EINSTEINKatalog darstellt.

Unser Ziel war es, zuvor unbekannte AGN im RASS-BSC-Katalog zu identifizieren und zu untersuchen, d.h. ihre Röntgenstrahlung sollte zur Entdeckung bisher unbekannter AGN benutzt werden. Daran schloss sich unmittelbar die Frage an, um welche Art von AGN es sich jeweils handelt, bzw. warum diese zuvor unentdeckt geblieben waren. Da es schon umfangreiche Suchprogramme für AGN in anderen Wellenlängenbereichen gegeben hatte (z.B. im UV), hätte es sich um aus rein statistischen Gründen unentdeckte oder aber um eine neue Spezies von AGN handeln können, die erst durch Röntgenemission ihre Natur als AGN offenbarten.

Das Selektionsverfahren besteht im Wesentlichen aus folgenden Schritten, die anschließend noch genauer beschrieben werden:

- Kreuzkorrelation RASS-BSC $\times$ PGC

- RASS-BSC: 18811 Röntgenquellen

- PGC: 73197 optische extragalaktische Quellen

- Kreuzkorrelation: 904 Röntgenquellen mit extragalaktischen Gegenstücken

- Auswahl kompakter Röntgenquellen, Entfernung von Galaxienhaufen

- Überprüfung bzgl. Vordergrundsternen mit Hilfe des DSS

- Entfernung bekannter AGN mit Hilfe der NED-Datenbank

- XGAL Sample: 198 unidentifizierte extragalaktische kompakte Röntgenquellen

Durch eine Kreuzkorrelation der 18811 Röntgenquellen des RASS-BSC mit den 73197 Galaxien des „Catalogue of Principal Galaxies“ PGC (Paturel et al. 1989) haben wir ein 
Sample von Galaxien zusammengestellt, die eine RASS-Zählrate von $\geq 0.05$ cts/s (entsprechend einer Röntgenleuchtkraft von $2 \cdot 10^{42} \mathrm{erg} \mathrm{s}^{-1}$ in $100 \mathrm{Mpc}$ Abstand) aufweisen. Als optimal hatte sich dabei ein Korrelationsradius von $100^{\prime \prime}$ erwiesen. Auf diese Weise erhielten wir eine Liste von 904 Röntgenquellen mit optischen extragalaktischen Gegenstücken.

Sodann haben wir uns auf kompakte Röntgenquellen beschränkt, wobei auch zahlreiche räumlich höher aufgelöste Röntgenbilder herangezogen wurden, die eigens zu diesem Zweck mit der HRI-Kamera des ROSAT-Satelliten aufgenommen wurden (s.a. Pietsch, Bischoff, et al. (1998)). Über HRI-Beobachtungen und deren Analyse haben wir in Bischoff \& Kollatschny (1994), Bischoff et al. (1995) und Bischoff et al. (1996) berichtet.

Kandidaten für Galaxienhaufen, bei denen die Röntgenemission hauptsächlich von ausgedehntem heißem Gas im Haufen stammt, wurden anhand der Röntgendaten wie in Zimmermann et al. (2001) beschrieben aussortiert.

Um eine eventuelle Kontamination der Röntgendaten durch Vordergrundsterne auszuschließen, wurden die entsprechenden Felder mit Hilfe des „Digitized Sky Survey“2 (DSS), einer digitalisierten optischen Durchmusterung des gesamten Himmels, überprüft.

Schließlich wurden bekannte AGN aus dem Sample entfernt. Dazu wurden alle bisher publizierten Informationen über verbliebenen Quellen mit Hilfe der „NASA/IPAC Extragalactic Database “3 (NED) herangezogen. NED ist die aktuellste und vollständigste Datenbank für extragalaktische Quellen und beinhaltet insbesondere auch die Galaxien des Veron-Kataloges.

Damit haben wir ein Sample von 198 Galaxien erhalten, die einen hohen Röntgenfluss zeigen (RASS-Zählrate $\geq 0.05 \mathrm{cts} / \mathrm{s}$ ), ohne dass dafür ein offensichtlicher Grund bekannt war. Für viele der Objekte lagen nicht einmal Messungen der Rotverschiebung vor. Um die genaue Natur dieser Quellen zu klären und den Grund dafür, dass sie zuvor unentdeckt geblieben waren, zu untersuchen, wurden diese über ihre Röntgenstrahlung selektierten Galaxien in einem zweiten Schritt mittels optischer Spektroskopie genauer analysiert.

Während der optischen Beobachtungen fanden sich etwa zehn Feldquellen, die sowohl die Selektionskriterien hinsichtlich ihrer Röntgenemission erfüllten, als auch eindeutig

\footnotetext{
${ }^{2}$ The Digitized Sky Survey was produced at the Space Telescope Science Institute under U.S. Government grant NAG W-2166. The images of these surveys are based on photographic data obtained using the Oschin Schmidt Telescope on Palomar Mountain and the UK Schmidt Telescope. The plates were processed into the present compressed digital form with the permission of these institutions.

${ }^{3}$ This research has made use of the NASA/IPAC Extragalactic Database (NED) which is operated by the Jet Propulsion Laboratory, California Institute of Technology, under contract with the National Aeronautics and Space Administration.
} 
extragalaktischer Natur und zuvor nicht katalogisiert waren. Diese Galaxien wurden in das Sample aufgenommen.

Die hier beschriebene Definition und Selektion des Samples haben wir bereits in mehreren Publikationen (Pietsch, Bischoff, et al. 1998, Bischoff et al. 1998, 1999) veröffentlicht.

\subsection{Optische Beobachtungskampagnen}

Ein wesentliches und entscheidendes Element dieser Arbeit ist die optische Spektroskopie. Von den wie in den beiden vorherigen Abschnitten beschrieben selektierten Galaxien wurden nur diejenigen in das endgültige Sample übernommen, von denen ein optisches Spektrum mit hinreichender Qualität für die vor allem in den folgenden Kapiteln 3 und 6 beschriebenen Analysen gewonnen werden konnte.

Für die Spektroskopie wurden zahlreiche Beobachtungskampagnen an den Observatorien Calar Alto/DSAZ in Spanien (CA), Europäische Südsternwarte/ESO in La Silla/Chile und McDonald Observatorium in Texas/USA (MDO) durchgeführt. Eine Übersicht über die Beobachtungen wird in Tabelle 2.1 gegeben.

Die VBLG untersuchen wir in parallelen Projekten auch hinsichtlich ihrer Variabilität (Dietrich, Bischoff \& Kollatschny 1993, Erkens et al. 1995, Zetzl 2004). Daher wurden für einen Teil der Objekte mehrere Spektren mit zeitlichen Abständen von Monaten bis Jahren aufgenommen. Für dieses Projekt wird das jeweils beste Spektrum benutzt. In Tabelle 2.1 sind nur diejenigen Beobachtungskampagnen aufgeführt, bei denen tatsächlich hier verwendete Spektren gewonnen wurde.

Die Kampagnen Oktober 1989, Juli 1990, August 1991 und August 1992 dienten primär der Spektroskopie von VBLG, diejenigen von November 1996, Juli 1997 und August 1997 der Spektroskopie der röntgenselektierten Galaxien und der Beobachtungsaufenthalt von Juni 1995 war hauptsächlich für ein drittes, externes Projekt bestimmt.

Bei einen erheblichen Teil der Beobachtungen waren die Wetterbedingungen nicht photometrisch. Die spektrale Kalibration wurde dadurch nicht wesentlich eingeschränkt, die absolute Flusskalibration hingegen kann Ungenauigkeiten aufweisen. Das ist für diese Arbeit nicht erheblich, da nahezu ausschließlich Flussverhältnisse verwendet und diskutiert werden, die von solchen Ungenauigkeiten unabhängig sind. Von den aufgelisteten Meßwerten sind einzig die gemessenen $\mathrm{H} \alpha$ - und $\mathrm{H} \beta$-Flüsse in der Tabelle $8.2 \mathrm{im}$ Anhang von solchen Ungenauigkeiten betroffen. Das Seeing lag in den meisten Fällen zwischen $1^{\prime \prime}$ und $2^{\prime \prime}$.

Es wurde jeweils ein Langspalt mit einer Breite von 2" (bzw. im November 1996 mit einer Breite von 1.5") verwendet. Damit ergaben sich spektrale Auflösungen von typischerweise $6 \AA$ bis $10 \AA \AA$. In den benutzten Beobachtungsanordnungen entsprach ein 
Tabelle 2.1: Übersicht über die optischen Beobachtungskampagnen dieses Projekts, aus denen Spektren Verwendung gefunden haben. Die ersten Kampagnen dienten vorwiegend der Spektroskopie der VBLG, die letzten drei (1996 und 1997) der Spektroskopie der röntgenselektierten Galaxien. Die Observatorien sind mit CA (DSAZ/Calar Alto), MDO (McDonald Observatorium/Mt. Locke) und ESO (Europäische Südsternwarte/La Silla) abgekürzt.

\begin{tabular}{llllll}
\hline \hline Kampagne & Nächte & Teleskop & Instrument & Detektor & $\lambda \lambda[\AA]$ \\
\hline 1989 Okt. & 28. & $2.2 \mathrm{~m} \mathrm{CA}$ & B\&C & RCA\#11 & $4100-7500$ \\
1989 Okt. & 29. & $2.2 \mathrm{~m} \mathrm{CA}$ & B\&C & RCA\#11 & $5400-9000$ \\
1989 Okt. & 30. & $2.2 \mathrm{~m} \mathrm{CA}$ & B\&C & RCA\#11 & $6800-10200$ \\
1990 Juli & 18. & $3.5 \mathrm{~m} \mathrm{CA}$ & B\&C & RCA\#11 & $4000-7300$ \\
1990 Juli & $20 ., 21$. & $3.5 \mathrm{~m} \mathrm{CA}$ & B\&C & RCA\#11 & $5000-8300$ \\
1990 Juli & 22. & $3.5 \mathrm{~m} \mathrm{CA}$ & B\&C & RCA\#11 & $6100-9400$ \\
1991 Aug. & 6. & $2.2 \mathrm{~m} \mathrm{CA}$ & B\&C & GEC\#13 & $5100-10700$ \\
1991 Aug. & 7. & $2.2 \mathrm{~m} \mathrm{CA}$ & B\&C & GEC\#13 & $4000-10500$ \\
1992 Aug. & $25 .-31$. & $2.2 \mathrm{~m} \mathrm{CA}$ & B\&C & TEK\#6 & $3800-9200$ \\
1995 Juni & 5. & $2.7 \mathrm{~m} \mathrm{MDO}$ & LCSpol & & $6400-7900$ \\
1996 Nov. & $2 .-6$. & $2.2 \mathrm{~m} \mathrm{ESO}$ & EFOSC2 & LORAL & $3800-8000$ \\
1997 Juli & $2 .-6$. & $2.2 \mathrm{~m} \mathrm{ESO}$ & EFOSC2 & LORAL & $4100-7400$ \\
1997 Aug. & $28.7 .-1.8$. & $2.2 \mathrm{~m} \mathrm{CA}$ & CAFOS & SITe\#1d & $4200-8200$ \\
\hline \hline
\end{tabular}

Detektorpixel in der Dispersionsrichtung zwischen $0.5 \AA$ und 1.4 $\AA$. Dies lag in allen Fällen weit unter der spektralen Auflösung der Apparatur und hatte somit für diese keine Relevanz.

Die primären Eigenschaften der verwendeten CCD-Detektoren sind in Tabelle 2.2 aufgelistet. Bei der Kampagne im Juli 1997 wurde der Detektor LORAL mit einem „Binning“ von $2 \times 2$ ausgelesen, ansonsten wurde keine „Binning“ angewandt. Zur Flusskalibration wurden folgende spektrophotometrische Standardsterne verwendet: BD $+28^{\circ} 4211, \mathrm{BD}+33^{\circ} 2642$, EG 274, Feige 24, Feige 110, G191B2B, HD 49798, HD 798, NGC 7293 (Zentralstern), Wolf 485A (Turnshek et al. 1990, Oke 1990). Die Gesamtbelichtungszeit der Galaxien wurde in den meisten Fällen auf zwei gleich lange Teilbelichtungen aufgeteilt, in einigen Fällen wurden aber auch eine einzige oder drei Teilbelichtungen durchgeführt. 
Tabelle 2.2: Liste der primären Eigenschaften der verwendeten CCD-Detektoren. Für Detektor LORAL sind auch die effektiven Parameter angegeben, die bei der Kampagne im Juli 1997 mit einem „Binning“ von $2 \times 2$ erreicht wurden; bei allen anderen Kampagnen wurde kein „Binning“ angewandt.

\begin{tabular}{lllll}
\hline \hline CCD & Dimension & \multicolumn{2}{c}{ Pixelgröße } & Binning \\
& \multicolumn{1}{c}{$[$ Pixel] } & {$[\mu \mathrm{m}]$} & $\left.{ }^{\prime \prime}\right]$ & \\
\hline RCA\#11 & $1024 \times 640$ & 15 & 0.85 & \\
GEC\#13 & $1152 \times 770$ & 22.5 & 1.32 & \\
TEK\#6 & $1024 \times 1024$ & 24 & 1.41 & \\
LORAL (96 Nov.) & $2048 \times 2048$ & 15 & 0.26 & \\
LORAL (97 Juli) & $1024 \times 1024$ & 30 & 0.52 & $2 \times 2$ \\
SITe\#1d & $2048 \times 2048$ & 24 & 0.53 & \\
\hline \hline
\end{tabular}

In Tabelle 2.3 ist das vollständige VBLG-Sample mit dem Beobachtungstermin und der Gesamtbelichtungszeit der letztlich verwendeten Spektren angegeben. Ferner ist auch jeweils die mittlere Balmerhalbwertsbreite (FWHM) aufgelistet, da es sich hierbei um einen entscheidenden Parameter hinsichtlich der Klassifikation der Galaxien als VBLG handelt. Die entsprechenden Daten für die röntgenselektierten Galaxien folgen mit zusätzlichen, für deren Klassifikation wichtigen Parametern, im folgenden Kapitel in Tabelle 3.1.

Zwei VBLG-Spektren hatten ein so geringes Signal/Rausch-Verhältnis, dass Spektren mehrerer Epochen gemittelt wurden: bei V396Her wurde eine Mittelung der Spektren von Juli 1990 und August 1992, bei 4U0241+61 derjenigen von Oktober 1989, Juli 1990, August 1991 und August 1992 vorgenommen. In diesen beiden Fällen wird jeweils Juli 1990 als Aufnahmedatum geführt und die Gesamtbelichtungszeit aller verwendeten Spektren angegeben. 
Tabelle 2.3: Liste der VBLG mit den Beobachtungsterminen sowie der Gesamtbelichtungszeit $t_{\exp }$ der verwendeten Spektren. Es ist auch bereits die mittlere Balmerhalbwertsbreite (FWHM) angegeben, deren Messung erst im Abschnitt 6.1 beschrieben wird, da sie ein entscheidendes Kriterium für die Klassifikation der VBLG ist. Die hier eingeführte Nummerierung der Galaxien wird in den folgenden Datentabellen beibehalten.

\begin{tabular}{rllcc}
\hline \hline Nr. & Name & $\begin{array}{l}\text { Beobachtungs- } \\
\text { termin }\end{array}$ & $\begin{array}{c}\mathrm{t}_{\text {exp }} \\
{[\mathrm{min} .]}\end{array}$ & $\begin{array}{c}\text { FWHM } \\
{\left[\mathrm{km} \mathrm{s}^{-1}\right]}\end{array}$ \\
\hline 1 & 1E04124-080 & 89Oct 28. & 50 & 5319 \\
2 & 1E0450.3 & 96Nov 5. & 30 & 15504 \\
3 & 1E1821+643 & 90Jul 22. & 88 & 6678 \\
4 & 1E2251-178 & 92Aug 27. & 50 & 9549 \\
5 & 3C109.0 & 89Oct 29. & 102 & 6249 \\
6 & 3C227 & 95Jun 5. & 60 & 10384 \\
7 & 3C323.1 & 91Aug 7. & 63 & 6337 \\
8 & 3C382 & 92Aug 26. & 50 & 13097 \\
9 & 3C390.3 & 91Aug 6. & 55 & 12042 \\
10 & 3C445 & 92Aug 29. & 60 & 7760 \\
11 & 4 C11.72 & 91Aug 7. & 60 & 10509 \\
12 & 4 C29.06 & 90Jul 20. & 70 & 12581 \\
13 & 4 C31.30 & 89Oct 29. & 60 & 12884 \\
14 & $4 C 31.63$ & 92Aug 25. & 54 & 6614 \\
15 & 4 U0241+61 & 90Jul 18. & 183 & 13516 \\
16 & Arp102B & 92Aug 30. & 50 & 14896 \\
17 & ESO 141-55 & 97Jul 3. & 10 & 5761 \\
18 & Mkn1152 & 92Aug 30. & 40 & 12949 \\
19 & Mkn304 & 90Jul 18. & 50 & 11244 \\
20 & Mkn380 & 89Oct 30. & 120 & 7041 \\
21 & Mkn876 & 92Aug 27. & 50 & 8400 \\
22 & Mkn926 & 92Aug 26. & 45 & 10855 \\
23 & Mkn975 & 89Oct 28. & 50 & 4914 \\
24 & NGC3516 & 92Aug 27. & 15 & 8873 \\
25 & PG0007+106 & 92Aug 29. & 50 & 5968 \\
26 & PG0026+12 & 90Jul 21. & 50 & 4983 \\
27 & PG0906+48 & 89Oct 29. & 50 & 4206 \\
28 & PG1411+442 & 91Aug 6. & 30 & 6117 \\
29 & PG1700+518 & 92Aug 26. & 60 & 9702 \\
30 & PKS0130+242 & 92Aug 29. & 30 & 12235 \\
\hline \hline & & & & \\
\hline
\end{tabular}




\begin{tabular}{lllcc}
\hline \hline Nr. & Name & $\begin{array}{l}\text { Beobachtungs- } \\
\text { termin }\end{array}$ & $\begin{array}{c}\mathrm{t}_{\text {exp }} \\
{[\text { min. }]}\end{array}$ & $\begin{array}{c}\text { FWHM } \\
{\left[\mathrm{km} \mathrm{s}^{-1}\right]}\end{array}$ \\
\hline 31 & PKS0133+207 & 89Oct 30. & 117 & 4620 \\
32 & PKS0159-117 & 92Aug 26. & 30 & 7205 \\
33 & PKS0214+108 & 92Aug 30. & 25 & 3051 \\
34 & PKS0231+022 & 92Aug 31. & 75 & 13799 \\
35 & PKS0405-12 & 89Oct 30. & 105 & 4399 \\
36 & PKS2128-123 & 89Oct 30. & 60 & 9695 \\
37 & PKS2135-147 & 92Aug 27. & 55 & 8563 \\
38 & PKS2141+175 & 90Jul 20. & 90 & 7251 \\
39 & PKS2349-01 & 92Aug 29. & 60 & 14643 \\
40 & S10721 & 91Aug 6. & 50 & 6350 \\
41 & TON202 & 91Aug 7. & 45 & 12636 \\
42 & TON256 & 91Aug 6. & 76 & 4381 \\
43 & UGC12163 & 92Aug 29. & 40 & 2209 \\
44 & V396Her & 90Jul 20. & 120 & 9320 \\
\hline \hline
\end{tabular}

\subsection{Datenreduktion}

Die aufgrund der zahlreichen Beobachtungskampagnen und der verschiedenen benutzten Instrumentierungen sehr aufwändige Reduktion der Spektren wurde in standardisierter Weise mit dem Softwarepaket MIDAS $S^{4}$ (Munich Image Data Analysis System) durchgeführt. Das Verfahren ist in Bischoff (1993) ausführlich beschrieben und wird im Folgenden nochmals zusammengefasst.

Die mit einem Halbleiterdetektor gemessene Zählrate $C(x, \lambda)$ setzt sich wie folgt aus ortsabhängigen $(\mathrm{x})$ und wellenlängenabhängigen $(\lambda)$ Termen zusammen:

$$
C(x, \lambda)=(I(x, \lambda) \cdot I E(\lambda) \cdot A E(\lambda) \cdot A p f(\lambda)+S k y(x, \lambda)) \cdot F F(x)+D(x)+D_{0}(x)
$$

mit

C gemessene Zählrate

I absolute Intensitätsverteilung des Beobachtungsobjektes

\footnotetext{
${ }^{4}$ European Southern Observatory Munich Image Data Analysis System is developed and maintained by the European Southern Observatory
} 


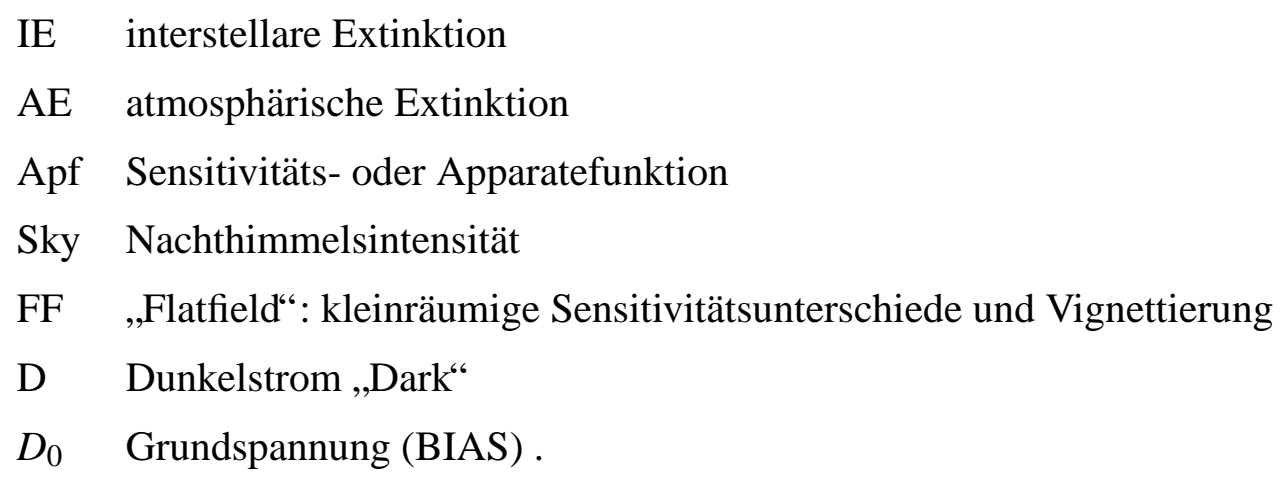

\section{BIAS und Dunkelstrom}

Der BIAS, der auf einer aus technischen Gründen ständig am Detektor anliegenden Grundspannung beruht, wurde mit Hilfe der eigens dafür registrierten BIASAufnahmen subtrahiert. Aus diesen Aufnahmen wurde auch das Ausleserauschen als Standardabweichung der Zählrate bestimmt. Bei geschlossenem Kameraverschluss aufgenommene sog. ,Darks“ dienten der Bestimmung des belichtungszeitabhängigen Dunkelstroms. Er war für die in dieser Arbeit verwendeten, relativ kurzen Belichtungszeiten immer weit unter dem Ausleserauschen und so gering, dass er nicht weiter berücksichtigt werden musste.

\section{„Flatfield“6}

Mittels sogenannter „Flatfields“, homogen ausgeleuchteter Aufnahmen, wurden sowohl kleinräumige Sensitivitätsunterschiede einzelner Detektorelemente (,Pixel“) wie auch die vorwiegend auf Vignettierung zurückzuführenden großskaligen Sensitivitätsunterschiede in der räumlichen Dimension (Horne 1986) des Detektors korrigiert.

\section{Nachthimmel}

„Cosmics“, lokal erhöhte Zählraten durch energiereiche Partikel z.B. aus der kosmischen Höhenstrahlung oder radioaktivem Zerfall, wurden durch eine Median-Filterung mit an das Rauschen der Aufnahme angepassten Parametern entfernt. Der Nachthimmel wurde durch Anpassung von Polynomen in der räumlichen Dimension des Detektors an objektfreie Bereiche links und rechts der Galaxien interpoliert und anschließend subtrahiert. 


\section{Extraktion}

Aus der zweidimensionalen Registrierung wurden eindimensionale Spektren ausgelesen, indem i.d.R. ein Bereich von $3^{\prime \prime}$ symmetrisch um das Zentrum des beobachteten Objektes (Pixel mit der maximalen Zählrate) räumlich zusammengefasst wurde.

\section{Wellenlängenkalibration}

Zur Wellenlängenkalibration wurden während der Beobachtungen Eichspektren von Vergleichslampen, in den meisten Fällen Helium-Argon-Lampen, aufgenommen. Die in den Eichspektren identifizierten Spektrallinien wurden mit einem Polynom angepasst, womit wiederum die Wellenlängenskala der Objektspektren kalibriert wurde.

\section{Extinktion}

Die sog. Luftmasse (,Airmass“) wurde nach Hardie (1962) berechnet und die atmosphärische Extinktion anschließend mit Hilfe der Extinktionskurve von La Silla/Chile (ESO Users Manual 1983) und der berechneten Airmass korrigiert. Die interstellare Extinktion wurde mit der Extinktionskurve von Savage \& Mathis (1979) korrigiert, wobei der Farbexzeß $\mathrm{E}_{B-V}$ nach Burstein \& Heiles $(1982,1984)$ bestimmt wurde.

\section{Sensitivitätsfunktion}

Mit beobachteten Spektren der Standardsterne BD $+28^{\circ} 4211$, BD $+33^{\circ} 2642$, EG 274, Feige 24, Feige 110, G191B2B, HD 49798, HD 798, NGC 7293 (Zentralstern), Wolf 485A und mit Hilfe der von Turnshek et al. (1990) sowie Oke (1990) tabellierten spektrophotometrischen Daten wurden die Sensitivitäts- bzw. Apparatefunktionen berechnet und damit die Flusskalibration der Objektspektren durchgeführt.

Für dieses Projekt sind etliche Reduktions- und Auswertungs-Programme innerhalb der MIDAS-Umgebung programmiert worden. Die wichtigsten, wie z.B. deblend.prg und detz.prg, werden an den entsprechenden Stellen in dieser Arbeit erwähnt und erläutert. 


\section{Kapitel 3}

\section{Optische Spektroskopie \& Klassifikation}

Während die VBLG als ein sehr spezieller Typ Aktiver Galaxien selektiert wurden und somit diesbezüglich keine Zweifel herrschten, konnte bei den röntgenselektierten Galaxien nur vermutet werden, dass es sich um AGN handelt. Die tatsächliche Natur dieser Quellen wurde mittels optischer Spektroskopie geklärt.

In diesem Kapitel wird das genaue Selektionsverfahren beschrieben: zunächst wird eine detaillierte Linienprofilanalyse der optischen Spektren vorgenommen, mit deren Ergebnissen dann die Klassifikation der Galaxien erfolgt. Anschließend werden die Resultate weiter analysiert und diskutiert.

\subsection{Linienprofilanalyse}

Die Linienprofile der Objekte des vorliegenden Samples sind teilweise sehr komplex aufgebaut. Insbesondere die Balmerlinien der VBLG setzen sich häufig aus mehreren, unterschiedlich breiten Komponenten zusammen, die im Falle von $\mathrm{H} \alpha$ noch von den schmalen Linien [N II] 26548,6584 , bei sehr breiten H $\alpha$-Profilen auch von [S II] $\lambda 6717,6731$ und [O I] $\lambda 6300,6364$ und im Falle von $\mathrm{H} \beta$ von [O III] $\lambda 4959,5007$ überlagert sein können.

Aus diesem Grunde wurden alle Messungen durch Anpassung von Gaußkurven, die den o.g. Linienkomponenten entsprechen, an die gesamten Linienkomplexe durchgeführt. Die Gaußfunktion hat gegenüber anderen Funktionen den Vorteil, dass sie mit nur drei Parametern beschrieben werden kann und erfahrungsgemäß gute Ergebnisse bei der Anpassung von Spektrallinien erzielt werden. Dieses Verfahren liefert für alle Komponenten die zentrale Wellenlänge, die Halbwertsbreite und den integrierten Fluss. Neben 
der Entfaltung überlagerter Linienprofilkomponenten hat dies den weiteren Vorteil, dass auch bei einfach strukturierten Linien stets das gesamte Profil zur Messung benutzt wird. Das führt gegenüber einer direkten Messung insbesondere bei Objekten mit geringem Signal/Rausch-Verhältnis zu robusteren Ergebnissen.

Konkret wurde wie folgt vorgegangen: Zuerst wurden für jeden Linienkomplex eine lineare Interpolation des Kontinuums und Anfangswerte für die direkt erkennbaren Linienkomponenten vorgegeben und daraus ein Fit errechnet. Zeigte sich dann, dass eine weitere Linienkomponente nötig ist, um das Gesamtprofil adäquat anzupassen, wurde eine solche hinzugefügt. Auf diese Weise musste häufig nach dem ersten Fit sofort eine breitere $\mathrm{H} \alpha$ - bzw. $\mathrm{H} \beta$-Komponente hinzugenommen werden. In seltenen Fällen musste dieser Schritt ein zweites Mal wiederholt werden.

Nur in einigen Ausnahmefällen ließ sich auch so kein zufriedenstellender Gesamtfit errechnen, d.h. der Gesamtfluss des Linienkomplexes und des Fits wichen um mehr als ca. 3\%-5\% voneinander ab. Dann wurden zuerst die gut anpassbaren Komponenten vom Linienkomplex subtrahiert und anschließend das Residuum mit einer weiteren Komponente angepasst. Auch dieser Schritt musste gegebenenfalls iteriert werden.

Der eigentliche Fit wurde mit dem $M I D A S$-Kommando DEBLEND/LINE aus dem Context SPEC errechnet. Dazu wurde als Umgebung die Procedure deblend.prg geschrieben, die Spektrum und Fits so darstellt, dass die Qualität der Fits sofort beurteilt werden kann. Außerdem archiviert sie die einzelnen Fits und das Gesamtergebnis, schreibt alle Fit-Parameter in automatisch weiterzuverarbeitende Tabellen und fügt entsprechende Deskriptoren in das bearbeitete Spektrum ein.

Ein typisches Beispiel für einen Fit ist in Abbildung 3.1 zu sehen, welche den $\mathrm{H} \alpha-$ Komplex der VBLG PKS 2349-01 mitsamt der angepassten Linienkomponenten zeigt. Die Berechnung und die Erstellung der Abbildung wurde mit deblend.prg durchgeführt.

Der Gesamtfit besteht aus vier schmalen Komponenten der Linien $\mathrm{H} \alpha$, [N II] 26584 und [S II] 6717,6731 sowie einer breiten und einer sehr breiten H $\alpha$-Komponente. Mit sechs solcher Linienkomponenten konnten für viele Objekte sehr gute Ergebnisse erzielt werden. Die zweite [N II] $\lambda 6548$-Linie ist zwar im Spektrum angedeutet, in der Intensität aber zu schwach um adäquat angepasst werden zu können - dieses traf auch auf die meisten anderen Objekte zu.

Alle Messergebnisse der Linienprofilanalyse sind in Tabelle 3.1 in diesem Kapitel und in Tabelle $8.2 \mathrm{im}$ Anhang aufgelistet.

\subsection{Klassifikation der Galaxien-Aktivität}

In diesem Abschnitt wird das Schema beschrieben, nach dem die Klassifikation der Galaxien hinsichtlich ihres Aktivitäts-Typs durchgeführt wurde. Dieses betrifft nur die 


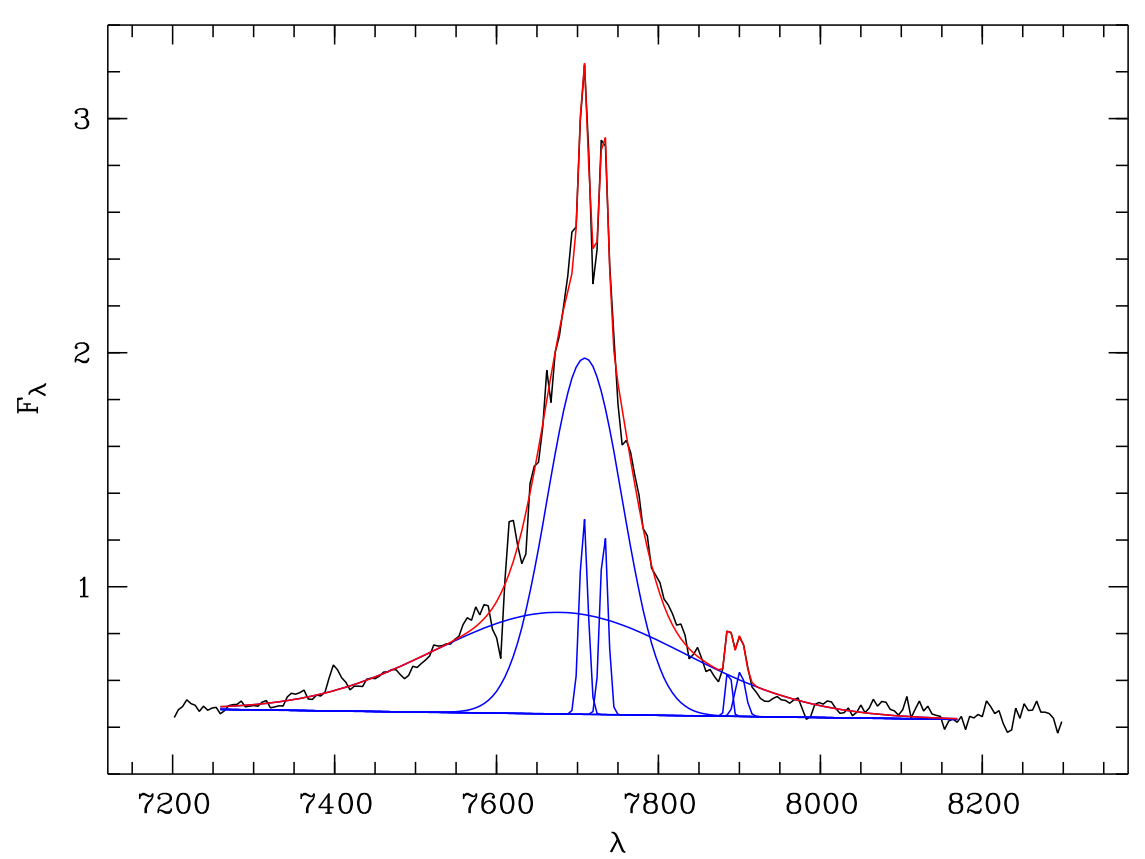

Abbildung 3.1: Die hier gezeigte Anpassung des rotverschobenen $\mathrm{H} \alpha-$ Komplexes der VBLG PKS 2349-01 ist ein typisches Beispiel für die in diesem Abschnitt beschriebene Linienprofilanalyse. Der Gesamtfit besteht aus vier schmalen Komponenten der Linien H $\alpha$, [N II] $\lambda 6584$ und [S II] 26717,6731 sowie einer breiten und einer sehr breiten H $\alpha$-Komponente. Die Linie [N II] $\lambda 6548$ ist zwar im Spektrum angedeutet, in der Intensität aber zu schwach um sinnvoll angepasst werden zu können. Das Kontinuum wurde linear interpoliert.

röntgenselektierten Galaxien, da deren Typ zuvor nicht bekannt war. Sie werden im Folgenden mit dem Kürzel XGAL bezeichnet.

Fundamentale AGN-Typen, die für das Gesamtverständnis Aktiver Galaxien und insbesondere das „Unified Model“ besonders wichtig sind, wurden bereits in Kapitel 1 besprochen und definiert. Einige tauchen im folgenden Klassifikationsschema wieder auf. Hinzu kommen weitere Typen bzw. Untertypen, denen in unserem Sample Bedeutung zukommt.

Zunächst wurde die Existenz von breiten Emissionslinien im Spektrum überprüft. War diese nicht sofort ersichtlich, so wurde sie mit der im vorherigen Abschnitt 3.1 beschriebenen Linienprofilanalyse geklärt. Objekte mit breiten Emissionslinien bzw. Emissionslinienkomponenten sind Typ-1 AGN, daher gehören auch die VBLG zu den Typ-1 AGN. 
- Seyfert-1

Die ursprüngliche Bezeichnung für Aktive Galaxien mit breiten Emissionslinien war Seyfert-1. Typ-1 AGN ist die modernere, allgemeinere Bezeichnung, die neben den klassischen Seyfert-1 Galaxien auch die QSO, die Untergruppe NarrowLine Seyfert-1 (NLS1) und die Übergangstypen Seyfert-1.5, Seyfert-1.8 und Seyfert-1.9 umfasst, die im Folgenden noch abgegrenzt werden.

- QSO

QSO und Seyfert-1 Galaxien sind anhand ihrer Spektren nicht zu unterscheiden; aus historischen Gründen werden die entfernteren, leuchtkräftigeren Objekte als QSO bezeichnet, die entsprechenden Quellen im nahen Universum hingegen als Seyfert-1, wobei es verschiedene Ansätze zur Abgrenzung gibt (z.B. Peterson (1988), Véron-Cetty \& Véron (2001)). Nur das Objekt RX J023454.8-293425 wird hier als QSO geführt, weil es mit einer Rotverschiebung von $\mathrm{z}=0.69$ alle anderen röntgenselektierten Galaxien, die Rotverschiebungen $\mathrm{z}<0.2$ aufweisen, bei weitem überragt (s. auch Abbildung 4.3). Damit einhergehend wurden bei diesem Objekt auch andere Emissionslinien (Mg II $\lambda 2798$ statt Balmerlinien) gemessen.

- NLS1

Narrow-Line Seyfert-1 Galaxien sind eine spezielle Unterklasse der Seyfert-1 Galaxien, heutzutage aber als eigenständiger AGN-Typ etabliert. Das wesentliche Kriterium besteht darin, dass in NLS1 Galaxien die Breite der Balmerlinien zwischen denen der klassischen Seyfert-1 und Seyfert-2 Galaxien liegt (etwa 500 - $2000 \mathrm{~km} \mathrm{~s}^{-1}$ ). Häufig zeigen NLS1 Galaxien auch Fe II-Emission (z.B. in den Multipletts bei $4570 \AA, 5190 \AA$ und $5300 \AA$ ) sowie Emission hochionisierter Linien wie [Fe VII] $\lambda 6087$ und [Fe X] $\lambda 6375$ (Osterbrock \& Pogge 1985, Goodrich 1989).

- Seyfert-2

Seyfert-2 Galaxien zeigen im Unterschied zu Seyfert-1 Galaxien nur schmale Emissionslinien. Die erlaubten Linien wie z.B. die Balmerlinien haben somit die gleiche Linienbreite wie die verbotenen Linien (ca. $500 \mathrm{~km} \mathrm{~s}^{-1}$ ), was erstmals von Khachikian \& Weedman (1971) festgestellt wurde. Die physikalischen Ursachen dieses rein phänomenologischen Unterschiedes gemäß der gängigen Modellvorstellungen wurden in Abschnitt 1.3 bereits diskutiert.

- Seyfert-1.5, $-1.8,-1.9$

Weiterhin hat Osterbrock (1977) Übergangstypen zwischen Seyfert-1 und Seyfert2 eingeführt, die in den erlaubten Linien sowohl breite als auch schmale Komponenten zeigen. Der detaillierteren Definition von Osterbrock (1989) folgend 
werden Seyfert-Galaxien, die in $\mathrm{H} \alpha$ und $\mathrm{H} \beta$ breite und schmale Komponenten ähnlicher Stärke zeigen, mit Seyfert-1.5 bezeichnet. Wenn $\mathrm{H} \alpha$ und $\mathrm{H} \beta$ im Vergleich zur schmalen Komponente nur sehr schwache breite Komponenten haben, werden sie Seyfert-1.8 genannt, wenn nur $\mathrm{H} \alpha$, nicht jedoch $\mathrm{H} \beta$ eine breite Komponente aufweist, handelt es sich um eine Seyfert-1.9 Galaxie.

Bei den beiden letztgenannten Typen Seyfert-1.8 und -1.9 waren in diesem Sample die breiten Linienkomponenten häufig nicht offensichtlich, sondern wurden erst durch die Linienprofilanalyse detektiert.

CGCG 425-034 zeigt in den Balmerlinien neben starken schmalen Linienkomponenten auch sehr breite Komponenten mit $\operatorname{FWHM}(\mathrm{H} \alpha)=6470 \mathrm{~km} \mathrm{~s}^{-1}$ und $\operatorname{FWHM}(\mathrm{H} \beta)=8710 \mathrm{~km} \mathrm{~s}^{-1}$. Diese breiten Linienkomponenten weisen Asymmetrien auf, wie sie auch für die VBLG charakteristisch sind. CGCG 425-034 wurde hier dennoch als Seyfert-1.5 klassifiziert, da das Objekt nicht aus dem rein spektral selektierten VBLG-Sample stammt.

Die Spektren aller Galaxien des Samples sind im Anhang (s. Abbildungen 8.1 und 8.2) bzw. in Pietsch, Bischoff, et al. (1998) abgebildet.

\section{- LINER}

Niedrig ionisierte Emissionsliniengalaxien (,Low Ionization Nuclear Emissionline Region“ LINER, Heckman (1980)) kann man in etwa 30\% aller hellen Galaxien finden (Ho, Filippenko \& Sargent 1997a). Auch wenn diese Objekte durch unterschiedliche Anregungsmechanismen dominiert werden (s. z.B. Filippenko (1996)), so ist doch inzwischen gezeigt worden, dass zumindest ein signifikanter Anteil dieser Gruppe einen schwachen Aktiven Kern enthält (Ho et al. 1997b). LINER werden gelegentlich auch als Seyfert-3 Galaxien bezeichnet.

\section{- H II-Galaxien}

Starburst- oder H II-Galaxien zeigen wie Seyfert-Galaxien auch Emissionslinien. Diese werden jedoch nicht wie bei letzteren durch einen Aktiven Kern angeregt, sondern allein durch Sternentstehungsausbrüche (,Starbursts“). Diese unterschiedlichen Anregungsmechanismen haben signifikant unterschiedliche Linienspektren zur Folge (Osterbrock 1989).

Anders als Seyfert-1 Galaxien und auch die Übergangstypen Seyfert-1.5, -1.8, -1.9 sind Seyfert-2, LINER und H II-Galaxien nicht unmittelbar zu unterschieden, da ihre Linienbreiten alle im gleichen Bereich von ca. $500 \mathrm{~km} \mathrm{~s}^{-1}$ FWHM liegen. Die optischen Spektren all dieser Objekte werden in der Regel gleichermaßen von Wasserstoff-, Sauerstoff- und Stickstofflinien dominiert. Wegen der unterschiedlichen Art der Anregung der linienemittierenden Regionen können die genannten Typen jedoch anhand der relativen Stärken der einzelnen Linien identifiziert werden. 


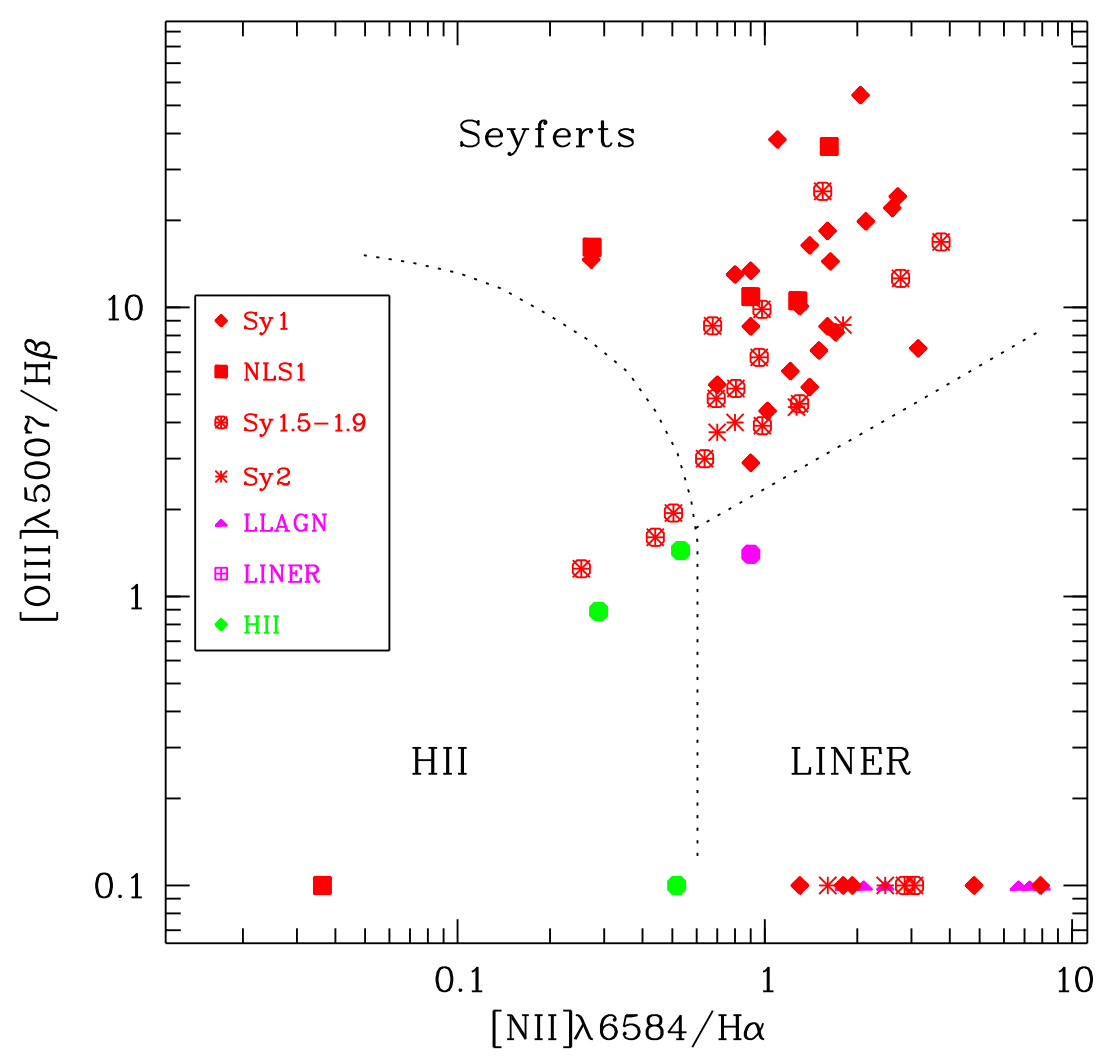

Abbildung 3.2: Diagramm diagnostischer Emissionslinien-Verhältnisse zur Klassifikation der Galaxien-Aktivität nach Baldwin, Phillips \& Terlevich (1981). Die gepunkteten Linien geben empirisch gefundene Abgrenzungen zwischen Seyfert-, LINER- und H II-Galaxien wieder. Naturgemäß sind in dieser Graphik nur diejenigen Objekte des XGAL-Teilsamples enthalten, für die schmale Linienkomponenten der entsprechenden vier Emissionslinien gemessen werden konnten - das war für 47 Galaxien der Fall. Bei einem arbiträren Ordinatenwert von 0.1 sind zusätzlich Objekte eingetragen, für die [N II] $\lambda 6584 / \mathrm{H} \alpha$, nicht jedoch $[\mathrm{O}$ III $] \lambda 5007 / \mathrm{H} \beta$ bestimmt werden konnte - das war für weitere 18 Galaxien der Fall. Bis auf zwei Ausnahmen kann für diese ausgeschlossen werden, dass es sich um H II-Galaxien handelt. Drei anhand breiter Linienkomponenten identifizierte Seyfert-Galaxien liegen im H II-Bereich. Die in der Legende dieser Abbildung erklärte Zeichenkodierung der einzelnen Galaxien-Typen wird auch in späteren Abbildungen verwendet werden.

Baldwin, Phillips \& Terlevich (1981) haben erstmals Diagramme diagnostischer Emissionslinienverhältnisse zur Klassifikation der Galaxien-Aktivität benutzt, die heute noch nach den Autoren Baldwin- oder auch BPT-Diagramme genannt werden. Abbildung 3.2 
zeigt ein solches Diagramm für das hier vorliegende Sample. Anhand der Verhältnisse der diagnostischen Emissionslinien $\mathrm{H} \alpha$ und [N II] $\lambda 6584$ sowie $\mathrm{H} \beta$ und [O III] $\lambda 5007$ kann zwischen Seyfert-, LINER- und Starburst-Galaxien (H II) unterschieden werden. Die eingezeichneten gepunkteten Linien geben empirisch gefundene Abgrenzungen zwischen diesen drei Galaxien-Typen wieder. Eine aktuelle Zusammenfassung spektroskopischer Liniendiagnostik findet sich bei Veilleux (2001).

Der Vergleich von Linienintensitäten ist natürlich nur sinnvoll, sofern diese Linien im gleichen Raumgebiet entstehen, also in der so genannten Narrow-Line Region (NLR). Daher sind naturgemäß in Abbildung 3.2 nur diejenigen Objekte des XGALTeilsamples enthalten, für die schmale Linienkomponenten der entsprechenden Emissionslinien gemessen werden konnten. Bei Seyfert-1 und insbesondere auch bei NLS1 Galaxien ohne messbare schmale Balmerkomponenten kann man das Schema nicht anwenden, diese Objekte sind allerdings auch anhand ihrer breiten Linienkomponenten sowieso zweifelsfrei klassifizierbar. Generell ist das Schema für Seyfert-1 Galaxien ungenauer als für Seyfert-2 Galaxien, da breite Balmerlinienkomponenten die Messung der schmalen Komponenten erschweren.

Insgesamt konnten für 47 Objekte sowohl die verbotenen Emissionslinien $[\mathrm{N}$ II] $\lambda 6584$ und [O III] $\lambda 5007$ als auch schmale Linienkomponenten der Balmerlinien $\mathrm{H} \alpha$ und $\mathrm{H} \beta$ gemessen werden. Der bei weitem größte Teil der Galaxien kann mit Hilfe dieses Diagramms sofort den Seyfert-Galaxien zugerechnet werden; unter den Objekten, für die alle vier genannten Emissionslinien gemessen werden konnten, liegen nur sechs nicht im Bereich der Seyfert-Galaxien.

Drei dieser Galaxien liegen im Bereich der H II-Objekte, obwohl sie als SeyfertGalaxien klassifiziert wurden: CGCG 196-064, CGCG 425-034 und Mkn 863 (in dieser Reihenfolge mit zunehmendem Verhältnis [N II] $\lambda 6584 / \mathrm{H} \alpha$ ). Alle drei Quellen haben eindeutig breite Balmerlininenkomponenten und sind daher zweifelsfrei SeyfertGalaxien. Andererseits sind die schmalen Balmerlininenkomponenten ungewöhnlich stark und führen dazu, dass die Galaxien im Baldwin-Diagramm im Bereich der H IIObjekte liegen.

Bei CGCG 196-064 sind die Linienprofile von H $\alpha$ und $\mathrm{H} \beta$ insgesamt relativ schmal und spektral nicht sehr gut aufgelöst; dies führt bei der Entfaltung der einzelnen Linien zu höheren Ungenauigkeiten und kann eine Ursache für die Lage im BaldwinDiagramm sein. Dasselbe gilt für Mkn 863 bezüglich H $\alpha$. Eine andere Fehlerquelle im Fall von CGCG 196-064 ist das deutlich unterdurchschnittliche Signal/Rausch-Verhältnis des Spektrums. Mkn 863 hingegen liegt sehr nahe an der Grenze zum Seyfert-Gebiet (Spektren s. Anhang).

Wichtig für zahlreiche weitere Abbildungen in allen folgenden Kapiteln ist die in der Legende der Abbildung 3.2 erklärte Zeichenkodierung der einzelnen Galaxien-Typen - sie wird in dieser Arbeit durchgehend benutzt werden. Hinzu kommen werden noch 

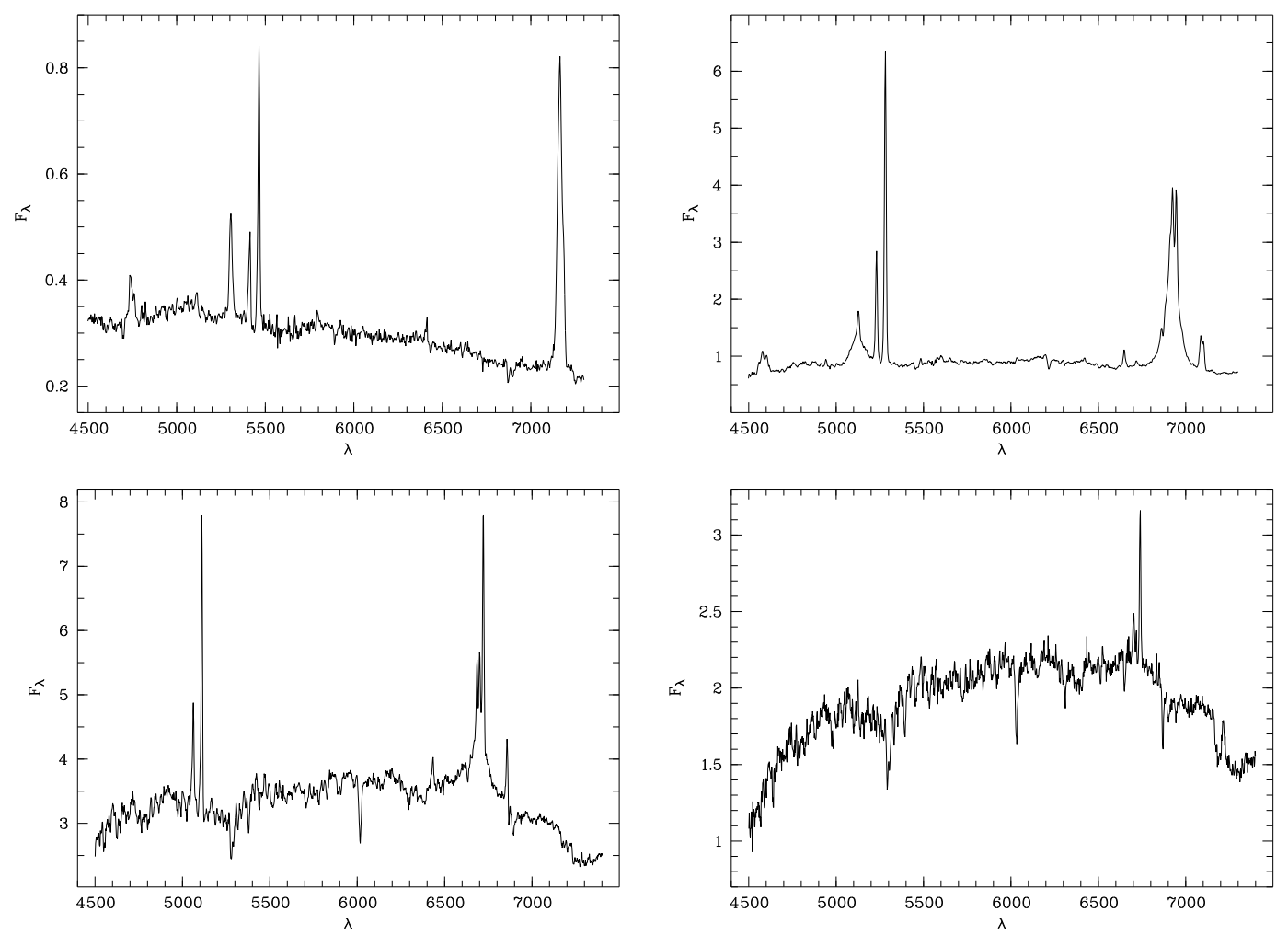

Abbildung 3.3: Typische Spektren verschiedener Galaxientypen: abgebildet sind Spektren der NLS1 Galaxie ESO 407-017 (oben links), der Seyfert-1.5 Galaxie ESO 381-007 (oben rechts), der Seyfert-1.9 Galaxie UGC 12040 (unten links) und der LLAGN Galaxie NGC 70 (unten rechts). Die im Text beschriebenen Charakteristika sind gut zu erkennen.

gefüllte blaue Kreise als Symbol für VBLG sowie offene hellblaue Rauten als Symbol für BL Lac Objekte.

\section{- LLAGN}

Für 18 Galaxien konnte zwar das Verhältnis [N II] $\lambda 6584 / \mathrm{H} \alpha$, nicht jedoch $[\mathrm{O}$ III $] \lambda 5007 / \mathrm{H} \beta$ gemessen werden. Diese Objekte sind bei einem Ordinatenwert $[\mathrm{O}$ III $] \lambda 5007 / \mathrm{H} \beta=0.1$ in Abbildung 3.2 eingezeichnet. Einige dieser Galaxien sind aufgrund breiter Balmerlinien eindeutig Seyfert-Galaxien. Für zwei Objekte (MCG 5-34-53 und AM 2354-304) konnten Obergrenzen für [O III] $\lambda 5007 / \mathrm{H} \beta$ abgeschätzt werden, aus denen folgt, dass es sich um H II-Objekte handelt. Für zwei weitere Objekte (PMN J0100-1517 und MCG -01-33-054) konnte zwar [O III] $\lambda 5007$, nicht jedoch $\mathrm{H} \beta$ detektiert werden; daraus wurde eine Untergrenze von $[\mathrm{O}$ III] $\lambda 5007 / \mathrm{H} \beta>10$ abgeschätzt, so dass es sich hierbei um Seyfert-2 
Galaxien handelt. Die übrigen könnten formal (schwache) Seyfert-2 Galaxien oder LINER sein.

Außerdem gibt es noch drei Galaxien, für die nur eine schwache Emission in $\mathrm{H} \alpha$ gemessen werden konnte und die daher in Abbildung 3.2 nicht eingezeichnet sind; auch diese Quellen können Seyfert-2 Galaxien oder LINER sein, zeigen aber in jedem Fall nur eine minimale Aktivität.

Aufgrund der geringen Leuchtkraft des Aktiven Kerns werden diese Quellen als „low-luminosity AGN“ (LLAGN) bezeichnet (Ho, Filippenko \& Sargent 1997a).

- Inaktive

Neben den Emissionsliniengalaxien gibt es einige Objekte im Sample, die ein gewöhnliches Absorptionslinienspektrum zeigen - es sind keinerlei Emissionslinien erkennbar. Hierbei handelt es sich um normale, nicht aktive bzw. inaktive Galaxien.

- BL Lac

Es befinden sich vier Quellen im Sample, die ein flaches, kontinuierliches Spektrum ohne Emissionslinien und ohne eindeutig identifizierbare Absorptionslinien zeigen. Diese Quellen wurden als BL Lac Objekte identifiziert, eine sehr spezielle und relativ seltene Klasse Aktiver Galaxien von denen man annimmt, dass ein auf uns zugerichteter Jet für das strukturlose Spektrum verantwortlich ist.

Abbildung 3.3 zeigt typische Beispiele für Spektren verschiedener Galaxientypen. Bei der NLS1 Galaxie ESO 407-017 oben links ist gut zu sehen, dass die Balmerlinien $\mathrm{H} \alpha$ und $\mathrm{H} \beta$ nur wenig breiter als die verbotenen Linien [O III] $\lambda 4959,5007$ sind. Die Seyfert1.5 Galaxie ESO 381-007 oben rechts zeigt sowohl schmale als auch breite Komponenten in den Balmerlinien, am besten zu erkennen in H $\beta$. Die Seyfert-1.9 Galaxie UGC 12040 unten links hat eine schwache breite $\mathrm{H} \alpha$-Komponente, jedoch keine breite $\mathrm{H} \beta$ Komponente. Der LLAGN NGC 70 unten rechts schließlich zeigt fast nur schwache

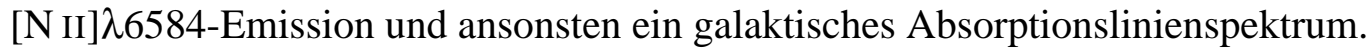

Ferner sind Beispiele für VBLG-Spektren (Abbildungen 2.1 und 3.1 in den vorherigen Abschnitten) und für ein Spektrum einer nicht aktiven Galaxie (Abbildung $3.5 \mathrm{im}$ übernächsten Abschnitt) abgebildet.

Die Ergebnisse dieses Klassifikationsschemas sind zusammen mit den Beobachtungsdaten und den Linienverhältnissen des Baldwin-Diagramms [N II] $\lambda 6584 / \mathrm{H} \alpha$ und [O III] $\lambda 5007 / \mathrm{H} \beta$ in Tabelle 3.1 aufgelistet. 
Tabelle 3.1: Identifikationen der röntgenselektierten Galaxien XGAL gemäß des in diesem Kapitel beschriebenen Klassifikationsschemas. Die Beobachtungstermine und Belichtungszeiten $t_{\text {exp }}$ der Spektren sowie die diagnostischen Linienverhältnisse des Baldwin-Diagramms (s. Abbildung 3.2) sind ebenfalls aufgelistet. Die hier eingeführte Nummerierung der Quellen wird wie auch bei den VBLG durchgängig beibehalten.

\begin{tabular}{|c|c|c|c|c|c|c|}
\hline $\mathrm{Nr}$. & Name & $\begin{array}{l}\text { Beob.- } \\
\text { termin }\end{array}$ & $\begin{array}{l}\mathrm{t}_{\text {exp }} / \\
\text { min. }\end{array}$ & $\frac{[\mathrm{N} \mathrm{II}]}{\mathrm{H} \alpha}$ & $\frac{[\mathrm{O} \text { III }]}{\mathrm{H} \beta}$ & Typ \\
\hline 45 & 1RXSJ201731.2-411452 & 97Jul 3. & 15 & & & BL Lac \\
\hline 46 & PHL 1389 & 96Nov 4. & 20 & & & BL Lac \\
\hline 47 & PMN J0630-2406 & 96Nov 2. & 10 & & & BL Lac \\
\hline 48 & RX J011232.8-320140 & 96Nov 3. & 15 & & & BL Lac \\
\hline 49 & RX J023454.8-293425 & 96Nov 4. & 50 & & & QSO \\
\hline 50 & AM 2354-304 E & 97Jul 5. & 20 & & & Sy1 \\
\hline 51 & CGCG 015-026 & 97Jul 5. & 30 & 2.13 & 19.86 & Sy 1 \\
\hline 52 & CGCG 049-106 & 97Jul 6. & 20 & 1.64 & 14.43 & Sy1 \\
\hline 53 & CGCG 433-025 & 96 Nov 3. & 30 & & 38.00 & Sy1 \\
\hline 54 & ESO 080- G 005 & 96Nov 2. & 30 & 0.80 & 13.00 & Sy1 \\
\hline 55 & ESO 104-IG041A & 97Jul 2. & 20 & & & Sy1 \\
\hline 56 & ESO 113- G 010 & 96Nov 2. & 15 & 1.40 & 5.30 & Sy1 \\
\hline 57 & ESO 15- IG 011 & 96Nov 4. & 30 & 0.70 & 5.40 & Sy1 \\
\hline 58 & ESO 209- G 012 & 96Nov 5. & 20 & 1.60 & 8.60 & Sy1 \\
\hline 59 & ESO 244- G 017 & 96Nov 4. & 15 & 1.50 & 7.10 & Sy1 \\
\hline 60 & ESO $328-36 \mathrm{~S}$ & 97Jul 2. & 20 & 1.93 & & Sy1 \\
\hline 61 & ESO 416- G 002 & 96Nov 4. & 25 & 2.60 & 22.10 & Sy1 \\
\hline 62 & ESO 490- IG 026 & 96Nov 4. & 15 & 1.40 & 16.40 & Sy1 \\
\hline 63 & ESO 511-30 & 97Jul 2. & 10 & 2.71 & 24.22 & Sy1 \\
\hline 64 & ESO 548- G 081 & 96 Nov 5. & 25 & 7.90 & & Sy1 \\
\hline 65 & ESO 552- G 039 & 96Nov 4. & 25 & 1.60 & 18.40 & Sy1 \\
\hline 66 & ESO 602- G 031 & 96Nov 2. & 30 & 1.30 & 10.10 & Sy1 \\
\hline 67 & Fairall 969 & 97Jul 2. & 60 & 3.16 & 7.22 & Sy1 \\
\hline 68 & $\mathrm{HCG} 4 \mathrm{a}$ & 96Nov 4. & 15 & 1.70 & 8.20 & Sy1 \\
\hline 69 & IC 5287 & 96Nov 4. & 30 & 4.80 & & Sy1 \\
\hline 70 & IRAS F20315-3047 & 97Jul 4. & 30 & 1.21 & 6.02 & Sy1 \\
\hline 71 & KUG 0128+328 & 97Aug 31. & 15 & 2.05 & 54.28 & Sy1 \\
\hline 72 & KUG 1618+410 & 97Aug 21. & 20 & 0.27 & 14.64 & Sy1 \\
\hline 73 & MCG -01-13-025 & 96Nov 5. & 20 & 0.90 & 2.90 & Sy1 \\
\hline 74 & MCG -02-12-050 & 96Nov 2. & 30 & 1.10 & 38.10 & Sy 1 \\
\hline
\end{tabular}


3.2 KLASSIFIKATION DER GALAXIEN-AKTIVITÄT

\begin{tabular}{|c|c|c|c|c|c|c|}
\hline Nr. & Name & $\begin{array}{l}\text { Beob.- } \\
\text { termin }\end{array}$ & $\begin{array}{l}\mathrm{t}_{\text {exp }} / \\
\text { min. }\end{array}$ & $\frac{[\mathrm{N} \mathrm{II}]}{\mathrm{H} \alpha}$ & $\frac{[\mathrm{O} \mathrm{III}]}{\mathrm{H} \beta}$ & Typ \\
\hline 75 & MCG -02-14-009 & 96Nov 2. & 15 & 0.90 & 8.60 & Sy1 \\
\hline 76 & MCG -02-59-006 & 96 Nov 5. & 15 & 1.30 & & Sy1 \\
\hline 77 & MCG 1-59-85 X884 & 97Jul 3. & 25 & & & Sy1 \\
\hline 78 & MCG 3-47-2 & 97Aug 29. & 29 & 1.02 & 4.38 & Sy1 \\
\hline 79 & NGC 1218 & 96Nov 2. & 25 & 21.50 & & Sy1 \\
\hline 80 & NGC 427 & 96Nov 3. & 45 & 1.80 & & Sy 1 \\
\hline 81 & PMN J0623-6436 & 96Nov 2. & 20 & & & Sy1 \\
\hline 82 & VIII Zw 36 & 96 Nov 3. & 15 & 0.90 & 13.40 & Sy1 \\
\hline 83 & CGCG 374-029 & 97Jul 3. & 20 & 1.62 & 36.07 & NLS1 \\
\hline 84 & CGCG 493-004 & 97Aug 30. & 20 & 0.04 & 0.41 & NLS1 \\
\hline 85 & ESO 407-17 & 97Jul 2. & 20 & & 1.38 & NLS1 \\
\hline 86 & IIZW 177 & 97Jul 3. & 30 & 1.28 & 10.55 & NLS1 \\
\hline 87 & KUG 1618+402 & 97Aug 31 . & 20 & 0.27 & 16.16 & NLS1 \\
\hline 88 & NGC 7158 & 96Nov 4. & 15 & 0.90 & 10.90 & NLS1 \\
\hline 89 & CGCG 163-074 & 97Aug 31. & 20 & 0.68 & 8.64 & Sy 1.5 \\
\hline 90 & CGCG 196-064 & 97Aug 1. & 20 & 0.25 & 1.25 & Sy1.5 \\
\hline 91 & CGCG 425-034 & 97Jul 5. & 40 & 0.44 & 1.60 & Sy 1.5 \\
\hline 92 & CGCG 493-002 & 97Aug 30. & 20 & 0.69 & 4.84 & Sy 1.5 \\
\hline 93 & ESO 381- 7 & 97Jul 3. & 20 & 0.98 & 9.85 & Sy 1.5 \\
\hline 94 & MCG 6-38-5 & 97Aug 31 . & 20 & 0.96 & 6.72 & Sy 1.5 \\
\hline 95 & MRK 863 & 97Jul 5. & 10 & 0.50 & 1.94 & Sy 1.5 \\
\hline 96 & NPM1G-16.0443 & 97Jul 3. & 20 & 0.80 & 5.24 & Sy1.5 \\
\hline 97 & CGCG 022-021 & 97Jul 2. & 20 & 0.98 & 3.90 & Sy 1.8 \\
\hline 98 & MCG -01-30-041 & 97Jul 5. & 20 & 0.64 & 3.00 & Sy 1.8 \\
\hline 99 & CGCG 023-021 & 97Jul 3. & 30 & 3.07 & & Sy 1.9 \\
\hline 100 & CGCG 21-63 & 97Jul 3. & 30 & 1.30 & 4.64 & Sy 1.9 \\
\hline 101 & CGCG 399-005 & 97Jul 2. & 20 & 2.77 & 12.61 & Sy 1.9 \\
\hline 102 & PKS 0018-19 & 97Jul 6. & 35 & 1.54 & 25.22 & Sy1.9 \\
\hline 103 & PKS 2331-24 & 97Jul 5. & 30 & & 6.86 & Sy1.9 \\
\hline 104 & UGC 12040 & 97Aug 29. & 30 & 2.85 & & Sy1.9 \\
\hline 105 & UGC 12282 & 97Aug 31. & 20 & 3.75 & 16.85 & Sy 1.9 \\
\hline 106 & ESO 409- 3 & 97Jul 2. & 20 & 1.27 & 4.52 & Sy2 \\
\hline 107 & MCG -01-05-031 & 96 Nov 6. & 15 & 0.70 & 3.70 & Sy2 \\
\hline 108 & MCG -01-33-054 & 97Jul 2. & 20 & 1.60 & & Sy2 \\
\hline 109 & PKS 1928-34 & 97Jul 2. & 30 & 1.80 & 8.70 & Sy2 \\
\hline 110 & PMN J0100-1517 & 97Jul 5. & 10 & 2.47 & & Sy2 \\
\hline 111 & UGC 3134 & 96Nov 5. & 15 & 0.80 & 4.00 & Sy2 \\
\hline
\end{tabular}




\begin{tabular}{|c|c|c|c|c|c|c|}
\hline Nr. & Name & $\begin{array}{l}\text { Beob.- } \\
\text { termin }\end{array}$ & $\begin{array}{l}\mathrm{t}_{\text {exp }} / \\
\text { min. }\end{array}$ & $\frac{[\mathrm{N} \text { II }]}{\mathrm{H} \alpha}$ & $\frac{[\mathrm{O} \text { III }]}{\mathrm{H} \beta}$ & Typ \\
\hline 112 & AM 0426-625 & 96 Nov 3. & 10 & & & LLAGN \\
\hline 113 & CGCG 375-033 & 97Jul 3. & 10 & & & LLAGN \\
\hline 114 & ESO 254- G 017 & 96 Nov 3. & 25 & 2.10 & & LLAGN \\
\hline 115 & ESO 399- 15 & 97Jul 3. & 20 & & & LLAGN \\
\hline 116 & ESO 45- 11 & 97Jul 2. & 30 & & & LLAGN \\
\hline 117 & IC 1867 & 96 Nov 5. & 10 & 6.70 & & LLAGN \\
\hline 118 & MCG 6-37-23 & 97Aug 30. & 10 & & & LLAGN \\
\hline 119 & NGC 5959 & 97Jul 3. & 10 & & & LLAGN \\
\hline 120 & NGC 6159 & 97Aug 1. & 10 & & & LLAGN \\
\hline 121 & NGC 6370 & 97Aug 30. & 10 & & & LLAGN \\
\hline 122 & NGC 70 & 97Aug 30. & 16 & 7.28 & & LLAGN \\
\hline 123 & NGC 71 & 97Aug 30. & 16 & 7.97 & & LLAGN \\
\hline 124 & NGC 7749 & 97Jul 3. & 10 & & & LLAGN \\
\hline 125 & UGC 11950 & 97Aug 30. & 10 & & & LLAGN \\
\hline 126 & UGC 12492 & 96Nov 5. & 10 & & & LLAGN \\
\hline 127 & UGC 32 & 97Aug 1. & 20 & 2.47 & & LLAGN \\
\hline 128 & VIIZW 608 & 97Aug 1. & 20 & 1.81 & & LLAGN \\
\hline 129 & ESO 122- IG 016 & 96 Nov 5. & 20 & & & LINER \\
\hline 130 & ESO 15- IG $011 \mathrm{~b}$ & 96Nov 4. & 15 & 0.90 & 1.40 & LINER \\
\hline 131 & NGC 1217 & 96Nov 2. & 10 & & & LINER \\
\hline 132 & ESO 267-13 & 97Jul 6. & 20 & 0.53 & 1.44 & HII \\
\hline 133 & MCG 5-34-53 & 97Aug 1. & 10 & 0.52 & & HII \\
\hline 134 & MCG 7-37-18 comp. & 97Aug 29. & 15 & 0.29 & 0.89 & HII \\
\hline 135 & CGCG 043-056 & 97Jul 6. & 10 & & & inaktiv \\
\hline 136 & ESO 120- G 023 & 96 Nov 5. & 10 & & & inaktiv \\
\hline 137 & ESO 122- IG 016 b & 96 Nov 5. & 20 & & & inaktiv \\
\hline 138 & ESO 184- 68 & 97Jul 2. & 10 & & & inaktiv \\
\hline 139 & ESO 290- $3 \mathrm{NE}$ & 97Jul 5. & 20 & & & inaktiv \\
\hline 140 & ESO 350- 15 & 97Jul 6. & 10 & & & inaktiv \\
\hline 141 & ESO 600- 14 & 97Jul 3. & 10 & & & inaktiv \\
\hline 142 & IC 4991 & 97Jul 3. & 10 & & & inaktiv \\
\hline 143 & MCG -07-47-032 & 97Jul 2. & 10 & & & inaktiv \\
\hline 144 & MCG 10-26-15 & 97Aug 30. & 10 & & & inaktiv \\
\hline 145 & MCG 13-12-22 & 97Aug 31. & 15 & & & inaktiv \\
\hline 146 & MCG 5-41-10 & 97Aug 30. & 10 & & & inaktiv \\
\hline 147 & MCG 7-37-18 & 97Aug 29. & 10 & & & inaktiv \\
\hline 148 & NGC 57 & 97Aug 31. & 10 & & & inaktiv \\
\hline
\end{tabular}




\begin{tabular}{lllcccc}
\hline \hline Nr. & Name & $\begin{array}{l}\text { Beob.- } \\
\text { termin }\end{array}$ & $\begin{array}{c}\mathrm{t}_{\text {exp }} / \\
\text { min. }\end{array}$ & $\frac{[\mathrm{N} \mathrm{II}]}{\mathrm{H} \alpha}$ & $\frac{[\mathrm{O} \text { III }]}{\mathrm{H} \beta}$ & Typ \\
\hline 149 & NGC 6160 & 97Aug 1. & 10 & & inaktiv \\
150 & NGC 6414 & 97Aug 28. & 30 & & inaktiv \\
151 & SGC 2005.3-5431 & 97Jul 3. & 10 & & inaktiv \\
152 & UGC 12804 & 97Aug 28. & 10 & & inaktiv \\
153 & UGC 716 & 96Nov 4. & 15 & & inaktiv \\
154 & VIIZW 608 comp. & 97Aug 1. & 20 & & inaktiv \\
\hline \hline
\end{tabular}


Tabelle 3.2: Analog zur Tabelle 3.1 sind hier die Linienverhältnisse [O I] $\lambda 6300 / \mathrm{H} \alpha$ und [S II] $\lambda 6717,6731 / \mathrm{H} \alpha$ aufgelistet, wobei bei letzteren die Summe der Flüsse der beiden Schwefellinien gemessen wurde. Da diese Linien i.d.R. schwächer sind als [N II] $\lambda_{6584}$, konnten sie bei weniger Objekten als $[\mathrm{N}$ II] $\lambda 6584 / \mathrm{H} \alpha$ bestimmt werden und sind zudem auch weniger genau. Anders als in Tabelle 3.1 sind hier nur diejenigen Galaxien aufgeführt, für die mindestens eines der Linienverhältnisse gemessen werden konnte.

\begin{tabular}{|c|c|c|c|c|}
\hline $\mathrm{Nr}$. & Name & $\frac{[\mathrm{O} \mathrm{I}] \lambda 6300}{\mathrm{H} \alpha}$ & $\frac{[\mathrm{S} \text { II }] \lambda 6717,6731}{\mathrm{H} \alpha}$ & Typ \\
\hline 50 & AM 2354-304 E & & 0.24 & Sy1 \\
\hline 51 & CGCG 015-026 & & 2.40 & Sy1 \\
\hline 52 & CGCG 049-106 & 0.20 & 1.48 & Sy1 \\
\hline 54 & ESO 080- G 005 & 0.10 & 0.30 & Sy1 \\
\hline 56 & ESO 113- G 010 & 0.10 & 0.40 & Sy 1 \\
\hline 57 & ESO 15- IG 011 & 0.10 & 0.50 & Sy1 \\
\hline 58 & ESO 209- G 012 & 0.20 & 0.80 & Sy1 \\
\hline 59 & ESO 244- G 017 & 0.20 & 0.30 & Sy1 \\
\hline 61 & ESO 416- G 002 & 0.60 & 1.00 & Sy1 \\
\hline 62 & ESO 490- IG 026 & 0.30 & 0.90 & Sy1 \\
\hline 63 & ESO 511-30 & 1.15 & & Sy 1 \\
\hline 64 & ESO 548- G 081 & 0.50 & 1.70 & Sy1 \\
\hline 65 & ESO 552- G 039 & 0.70 & 1.20 & Sy1 \\
\hline 66 & ESO 602- G 031 & 0.10 & 0.40 & Sy1 \\
\hline 67 & Fairall 969 & 0.38 & 1.21 & Sy1 \\
\hline 68 & HCG 4a & 0.20 & 0.70 & Sy1 \\
\hline 69 & IC 5287 & & 1.80 & Sy1 \\
\hline 70 & IRAS F20315-3047 & 0.26 & 0.65 & Sy1 \\
\hline 71 & KUG 0128+328 & 0.91 & 0.95 & Sy1 \\
\hline 72 & KUG $1618+410$ & 0.06 & 0.31 & Sy1 \\
\hline 73 & MCG -01-13-025 & 0.40 & 0.30 & Sy1 \\
\hline 74 & MCG -02-12-050 & 0.50 & 1.10 & Sy1 \\
\hline 75 & MCG -02-14-009 & & 0.20 & Sy1 \\
\hline 76 & MCG -02-59-006 & & 0.10 & Sy1 \\
\hline 78 & MCG 3-47-2 & & 0.40 & Sy1 \\
\hline 79 & NGC 1218 & & 9.50 & Sy 1 \\
\hline 80 & NGC 427 & 0.80 & 1.10 & Sy1 \\
\hline 82 & VIII Zw 36 & 0.20 & 0.50 & Sy1 \\
\hline 83 & CGCG 374-029 & & 2.42 & NLS1 \\
\hline
\end{tabular}


3.2 KLASSIFIKATION DER GALAXIEN-AKTIVITÄT

\begin{tabular}{|c|c|c|c|c|}
\hline Nr. & Name & $\frac{[\mathrm{O} \mathrm{I}] \lambda 6300}{\mathrm{H} \alpha}$ & $\frac{[\mathrm{S} \text { II }] \lambda 6717,6731}{\mathrm{H} \alpha}$ & Typ \\
\hline 84 & CGCG 493-004 & 0.01 & 0.09 & NLS1 \\
\hline 86 & IIZW 177 & & 0.33 & NLS1 \\
\hline 87 & KUG 1618+402 & 0.08 & 0.23 & NLS1 \\
\hline 88 & NGC 7158 & 0.10 & 0.10 & NLS1 \\
\hline 89 & CGCG 163-074 & 0.12 & 0.42 & Sy1.5 \\
\hline 90 & CGCG 196-064 & & 0.16 & Sy1.5 \\
\hline 91 & CGCG 425-034 & 0.03 & 0.22 & Sy 1.5 \\
\hline 92 & CGCG 493-002 & 0.08 & 0.34 & Sy 1.5 \\
\hline 93 & ESO 381- 7 & 0.15 & 0.59 & Sy 1.5 \\
\hline 94 & MCG 6-38-5 & 0.11 & 0.27 & Sy 1.5 \\
\hline 95 & MRK 863 & & 0.14 & Sy 1.5 \\
\hline 96 & NPM1G-16.0443 & & 0.43 & Sy1.5 \\
\hline 97 & CGCG 022-021 & 0.07 & 0.21 & Sy1.8 \\
\hline 98 & MCG -01-30-041 & 0.05 & 0.33 & Sy 1.8 \\
\hline 99 & CGCG 023-021 & & 1.41 & Sy1.9 \\
\hline 100 & CGCG 21-63 & 0.76 & 0.87 & Sy1.9 \\
\hline 101 & CGCG 399-005 & 0.74 & 0.77 & Sy1.9 \\
\hline 102 & PKS 0018-19 & & 1.11 & Sy1.9 \\
\hline 104 & UGC 12040 & 0.57 & 0.82 & Sy1.9 \\
\hline 105 & UGC 12282 & 0.46 & 1.33 & Sy1.9 \\
\hline 106 & ESO 409- 3 & 0.42 & 0.43 & Sy2 \\
\hline 107 & MCG -01-05-031 & 0.20 & 0.40 & Sy2 \\
\hline 108 & MCG -01-33-054 & & 0.58 & Sy2 \\
\hline 109 & PKS 1928-34 & & 0.97 & Sy2 \\
\hline 110 & PMN J0100-1517 & 0.30 & 1.30 & Sy2 \\
\hline 111 & UGC 3134 & 0.10 & 0.40 & Sy2 \\
\hline 114 & ESO 254- G 017 & & 1.20 & LLAGN \\
\hline 117 & IC 1867 & 0.80 & & LLAGN \\
\hline 130 & ESO 15- IG 011 b & 0.20 & 0.60 & LINER \\
\hline 132 & ESO 267-13 & & 0.20 & HII \\
\hline 133 & MCG 5-34-53 & & 0.48 & HII \\
\hline 134 & MCG 7-37-18 comp. & 0.08 & 0.44 & $\mathrm{HII}$ \\
\hline
\end{tabular}




\subsection{Häufigkeitsverteilung der AGN-Typen}

Im Folgenden soll auf die Häufigkeitsverteilung der verschiedenen AGN-Typen eingegangen werden, die sich aus dem im vorherigen Abschnitt beschriebenen Klassifikationsschema ergibt. Diese Häufigkeiten sind in Tabelle 3.3 sowohl absolut als auch relativ aufgelistet.

Zunächst sind in der linken Spalte alle bisher betrachteten AGN-Typen mit ihren jeweiligen Häufigkeiten einzeln aufgeführt. Es fällt sofort auf, dass einige Typen nur sehr selten vertreten sind, so sind z.B. nur ein QSO und zwei Seyfert-1.8 Galaxien im Sample. Weil außerdem gewisse Typen sehr eng miteinander verwandt sind, wurden sie wie folgt zu Gruppen zusammengefasst und in der mittleren Spalte der Tabelle 3.3 aufgeführt.

QSO, Seyfert-1 und NLS1 Galaxien gehören alle zu den Typ-1 AGN, d.h. sie haben eine beobachtbare Broad-Line Region und zeigen daher in ihren Spektren breite Emissionslinien. Die drei Übergangstypen Seyfert-1.5, Seyfert-1.8 und Seyfert-1.9, die sich durch das Verhältnis von schmaler zu breiter Linienkomponente von $\mathrm{H} \alpha$ und $\mathrm{H} \beta$ unterscheiden, wurden zu einer weiteren Gruppe zusammengefasst. Und letztlich wurden auch Seyfert-2, LLAGN und LINER-Galaxien in einer Gruppe gebündelt - wie oben bereits beschrieben, konnten die LLAGN, die anteilsmäßig etwa 65\% dieser Gruppe ausmachen, hinsichtlich ihres optischen Emissionslinienspektrums sowieso nicht eindeutig von Seyfert-2 oder LINER-Galaxien abgegrenzt werden.

Zuletzt wurden wiederum die drei obigen Gruppen zu Typ-1,2,3 AGN zusammengefasst. Ausgenommen blieben folglich nur die VBLG - formal Seyfert-1 Galaxien, die hier aber aufgrund ihrer besonderen Selektion separat betrachtet werden sollen -, die BL Lac, ein sehr spezieller AGN-Typ, sowie die H II- und die inaktiven Galaxien, bei denen es sich nicht um AGN handelt.

Um diese Häufigkeitsverteilung der verschiedenen AGN-Typen besser zu illustrieren, wurde sie in Abbildung 3.4 in Form eines Balkendiagramms graphisch dargestellt. Die primären, breiten Balken bezeichnen BL Lac (0), VBLG (0.5), Seyfert-1 bzw. Typ1AGN (1), Seyfert-1.5, -1.8 und -1.9 (1.5), Seyfert-2 (2), LLAGN (2.5) LINER bzw. Seyfert-3 (3), H II (4) und inaktive Galaxien (5). Die in Klammern angegebenen Zahlen bezeichnen die Position in diesem Diagramm und definieren damit gleichzeitig eine Typen-Sequenz, die auch im Folgenden weiter benutzt und diskutiert werden wird. Sie lehnt sich an die übliche Sequenz Seyfert-1, Seyfert-2, Seyfert-3/LINER an und soll diese zunächst rein formell erweitern.

Auch in Abbildung 3.4 ist der Balken für Typ-1 AGN/Sy-1 nochmals unterteilt in QSO, Seyfert-1 und NLS1 sowie derjenige für die Übergangstypen Seyfert-1.5 . . 1.9 in die drei einzelnen Typen.

Zusammenfassend kann festgehalten werden, dass das Gesamtsample aus vier größeren Gruppen Aktiver Galaxien besteht: den eigens selektierten VBLG, den Typ-1 AGN, 
Tabelle 3.3: Übersicht über die absoluten und prozentualen Häufigkeiten der einzelnen AGN-Typen. In der ersten Spalte ist die Häufigkeit jedes AGN-Typs aufgeschlüsselt, in Spalte zwei sind einige Typen zu Gruppen zusammengefasst, in der dritten Spalte sind wiederum mehrere Gruppen zusammengefasst (s. Text). Da die Prozentzahlen auf ganze Zahlen gerundet sind, ergeben sie in der Summe nicht zwangsläufig exakt $100 \%$.

\begin{tabular}{|c|c|c|c|c|c|c|c|c|}
\hline Тур & $\begin{array}{l}\text { Häu } \\
\text { abs. }\end{array}$ & $\begin{array}{r}\text { gkeit } \\
\text { rel. }\end{array}$ & Gruppe & $\begin{array}{l}\text { Häuf } \\
\text { abs. }\end{array}$ & $\begin{array}{r}\text { gkeit } \\
\text { rel. }\end{array}$ & Klasse & $\begin{array}{l}\text { Häu } \\
\text { abs. }\end{array}$ & $\begin{array}{r}\text { gkeit } \\
\text { rel. }\end{array}$ \\
\hline BL Lac & 4 & $3 \%$ & BL Lac & 4 & $3 \%$ & BL Lac & 4 & $3 \%$ \\
\hline VBLG & 44 & $29 \%$ & VBLG & 44 & $29 \%$ & VBLG & 44 & $29 \%$ \\
\hline QSO & 1 & $1 \%$ & & & & \multirow{9}{*}{$\begin{array}{l}\text { Typ-1,2,3 } \\
\text { AGN }\end{array}$} & \multirow{9}{*}{83} & \multirow{9}{*}{$54 \%$} \\
\hline Sy1 & 33 & $21 \%$ & Typ-1 AGN & 40 & $26 \%$ & & & \\
\hline NLS1 & 6 & $4 \%$ & & & & & & \\
\hline Sy 1.5 & 8 & $5 \%$ & \multirow{3}{*}{ Sy $1.5 \ldots 1.9$} & \multirow{3}{*}{17} & \multirow{3}{*}{$11 \%$} & & & \\
\hline Sy 1.8 & 2 & $1 \%$ & & & & & & \\
\hline Sy1.9 & 7 & $5 \%$ & & & & & & \\
\hline Sy2 & 6 & $4 \%$ & \multirow{3}{*}{$\begin{array}{l}\text { Sy2/LINER/ } \\
\text { LLAGN }\end{array}$} & \multirow{3}{*}{26} & \multirow{3}{*}{$17 \%$} & & & \\
\hline LLAGN & 17 & $11 \%$ & & & & & & \\
\hline LINER & 3 & $2 \%$ & & & & & & \\
\hline H II & 3 & $2 \%$ & H II & 3 & $2 \%$ & H II & 3 & $2 \%$ \\
\hline inaktiv & 20 & $13 \%$ & inaktiv & 20 & $13 \%$ & inaktiv & 20 & $13 \%$ \\
\hline \multicolumn{3}{|c|}{154} & \multicolumn{3}{|c|}{154} & \multicolumn{3}{|c|}{154} \\
\hline
\end{tabular}

den Übergangstypen Seyfert-1.5,-1.8,-1.9 und der Gruppe Seyfert-2/LINER/LLAGN. BL Lac Objekte und H II-Galaxien spielen anzahlmäßig mit vier bzw. drei Quellen keine Rolle.

Neben den H II-Galaxien gibt es außerdem noch inaktive Galaxien, von denen sich 20 unter den insgesamt 110 röntgenselektierten Galaxien befinden. Das bedeutet andererseits, dass $79 \%$ aller selektierten Quellen neue, d.h. zuvor nicht bekannte AGN sind. Damit hat sich das Selektionsverfahren als äußerst effizient zum Auffinden neuer AGN gezeigt, unsere Suchstrategie war somit sehr erfolgreich. 


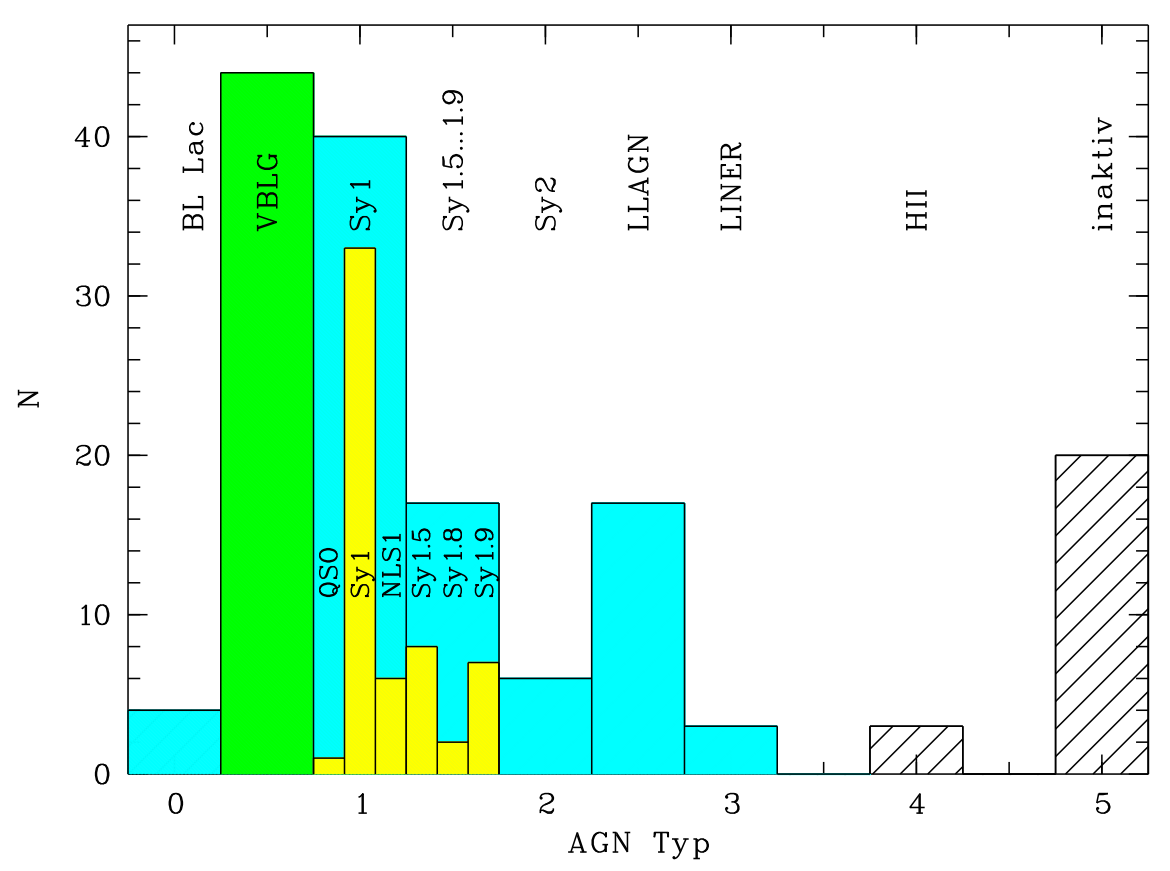

Abbildung 3.4: Häufigkeitsverteilung der Galaxien-Typen für das Gesamtsample (154 Quellen, s. Tabelle 3.3). Die Typ-1 AGN sind nochmals in QSO, Seyfert-1 und NLS1, die Übergangstypen Seyfert-1.5...1.9 in Sy-1.5, Sy-1.8 und Sy-1.9 aufgeschlüsselt, d.h. die Summe der Galaxien der jeweils drei Untertypen entspricht der Anzahl der Objekte in der übergeordneten Gruppe. Der hier auf der Abszisse abgetragene Typ-Parameter der verschiedenen AGN-Typen wird im Folgenden weiter verwendet.

\subsection{Rotverschiebung der Quellen}

Bisher wurde die Bezeichnung XGAL für alle röntgenselektierten Galaxien verwendet. Im Folgenden werden zusätzlich die Kürzel XAGN für alle röntgenselektierten AGN (also XGAL ohne H II- und inaktive Galaxien) sowie XAGN-1 für alle röntgenselektierten Typ-1 XAGN verwendet, so dass zur detaillierten Betrachtung des XGAL-Samples diese beiden Untergruppen zur Verfügung stehen. Das Teilsample VBLG bleibt davon natürlich unberührt.

Für die Aktiven Galaxien wurde die Rotverschiebung anhand der Emissionslinien gemessen. Für Quellen mit schmalen Linien wurden diese für die Analyse verwendet, da ihre zentralen Wellenlängen zum einen sehr viel genauer zu bestimmen sind als die der oft asymmetrischen und aus mehreren Komponenten bestehenden breiten Linien, und da zum anderen die breiten Komponenten neben der Systemgeschwindigkeit der Galaxie zusätzlich durch die Kinematik aufgrund des großen zentralen Gravitationspo- 


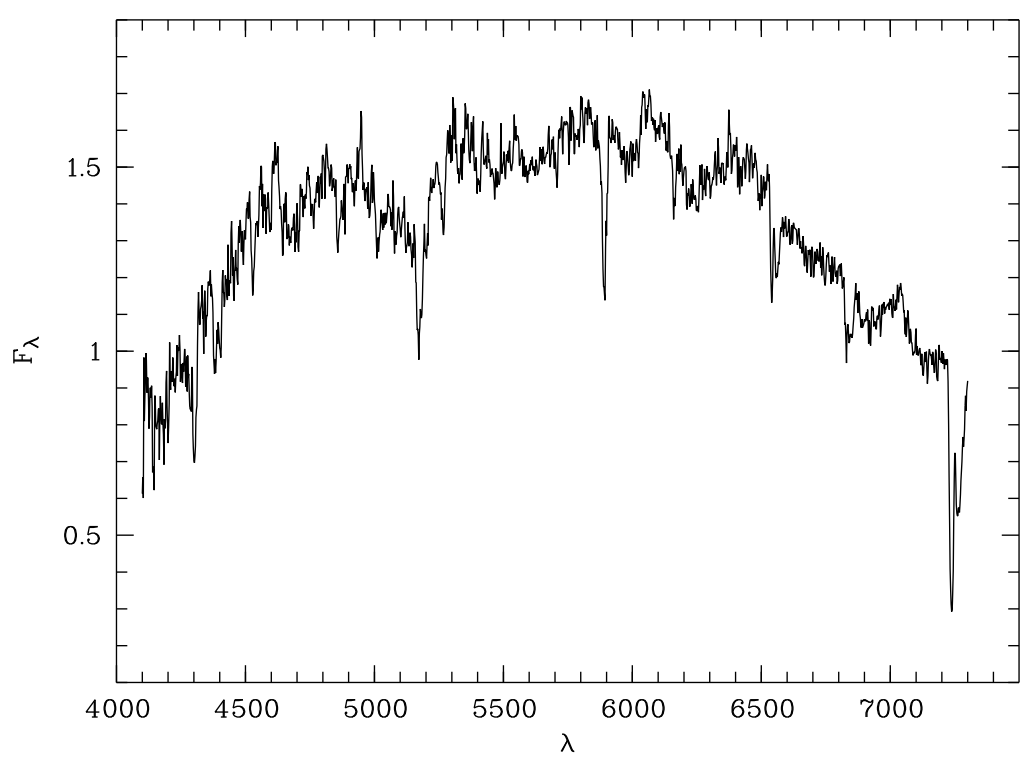

\begin{abstract}
Abbildung 3.5: Absorptionslinienspektrum der nicht aktiven Galaxie MCG +0737-018. Das Spektrum dieser Galaxie mit einer Rotverschiebung von $\mathrm{z}=0.051$ wurde ins Ruhesystem transformiert. Die stärksten galaktischen Absorptionen sind das G-Band bei $\lambda 4304, \mathrm{Mg}$ I $\lambda 5175$ und $\mathrm{Na}$ I D $\lambda 5892$, die starken Absorptionen jenseits von $6500 \AA ̊$ Å entstammen der Erdatmosphäre.
\end{abstract}

tenzials beeinflusst sind. Ferner kann zumindest bei sehr breiten Linienkomponenten die Gravitationsrotverschiebung einen zusätzlichen Beitrag leisten (z.B. Bischoff 1993, Kollatschny \& Bischoff 2002).

Die Zentralwellenlängen $\lambda$ der Emissionslinien wurden wie in Abschnitt 3.1 beschrieben im Rahmen der Linienprofilanalyse gemessen. Sodann wurde für jede Linie die Rotverschiebung

$$
z=\frac{\lambda}{\lambda_{0}}-1
$$

berechnet, wobei $\lambda_{0}$ die Laborwellenlänge der jeweiligen Linie ist. Die Systemrotverschiebung der Galaxie wurde dann als Mittelwert aus allen gemessenen Linien bestimmt. Auf diese Weise fielen auch Fehlmessungen einzelner Linien sofort durch eine abweichende Rotverschiebung auf und konnten unmittelbar korrigiert werden.

Für die nicht aktiven Galaxien ist dieses Verfahren mangels Emissionslinien naturgemäß nicht anwendbar; stattdessen wurde hier die Rotverschiebung mittels der galaktischen Absorptionslinien gemessen.

Abbildung 3.5 zeigt als Beispiel das Spektrum der Galaxie MCG+07-37-018, das von 
der ursprünglichen Rotverschiebung von $\mathrm{z}=0.051$ ins Ruhesystem transformiert wurde. Charakteristisch sind insbesondere die starken Absorptionen des G-Bandes bei $\lambda 4304$, von $\mathrm{Mg}$ I $\lambda 5175$ und $\mathrm{Na}$ I D $\lambda 5892$. Die starken Absorptionen jenseits von $6500 \AA$ sind atmosphärischer Natur (A- und B-Band Absorption) und daher keine Eigenschaft der Galaxie.

Zunächst wurde nun ein Absorptionslinienspektrum mit hohem Signal/Rausch-Verhältnis ausgewählt, bei dem die Rotverschiebung aus mehreren Linien mit sehr guter Übereinstimmung gemessen werden konnte. Dieses Spektrum wurde ins Ruhesystem ( $\mathrm{z}=0$ ) transformiert und bildet fortan das Referenzspektrum. Anschließend wurde die Rotverschiebung aller anderen Spektren durch Verschiebung und Vergleich mit Hilfe einer Kreuzkorrelation bestimmt.

Dieses Verfahren ist insbesondere bei Spektren mit niedrigerem Signal/Rausch-Verhältnis wesentlich genauer als eine direkte Messung, da immer das gesamte Spektrum mit zahlreichen, auch schwachen Absorptionslinien analysiert wird und nicht nur die wenigen, leicht identifizierbaren Hauptlinien.

Zur Durchführung dieser Methode wurde die MIDAS-Routine detz.prg entwickelt. Diese interpoliert zunächst interaktiv im zu messenden Spektrum und im Referenzspektrum die atmosphärischen Linien, da sich diese nicht im Wellenlängensystem der Galaxien befinden. Anschließend wird das Kontinuum normalisiert, da ansonsten der großräumige Kontinuumsverlauf die nachfolgende Kreuzkorrelation dominieren würde. Dazu wurde eine sog. ,Spline“-Funktion angepasst (Dierckxx 1982) und dann durch diese dividiert. Schließlich wird die Wellenlängenskala der Spektren logarithmiert; das vereinfacht den folgenden Arbeitsschritt, da nun allein mit linearen Verschiebungen die logarithmischen Spektren zur Deckung gebracht werden können. Letzteres geschieht automatisch im Rahmen einer Kreuzkorrelationsanalyse beider Spektren.

Aus der Position des Maximums der Korrelation läßt sich unmittelbar $\log (1+z)$ entnehmen und somit sofort die Rotverschiebung z des zu messenden Spektrums bestimmen. Weiterhin wird das ins Ruhesystem transformierte Spektrum errechnet. Wiederholt man mit diesem die Kreuzkorrelation mit nun sehr viel kleineren Wellenlängeninkrementen, so kann man die Genauigkeit der Rotverschiebungsbestimmung noch erheblich steigern.

Auf diese Weise konnten mit Hilfe von detz.prg die Rotverschiebungen der Absorptionsliniengalaxien in konsistenter Weise und mit großer Genauigkeit gemessen werden.

Auch ein Teil der Emissionsliniengalaxien zeigt deutliche Absorptionslinien der unterliegenden Galaxie und zwar umso deutlicher, je schwächer das überlagerte nichtthermische Kontinuum des Aktiven Kerns ist. In einem zweiten Schritt wurde detz.prg dahingehend erweitert, dass auch Emissionslinien interaktiv interpoliert werden konnten und somit anschließend das Programm die Rotverschiebung der unterliegenden Galaxie aus den Absorptionslinien messen konnte. 


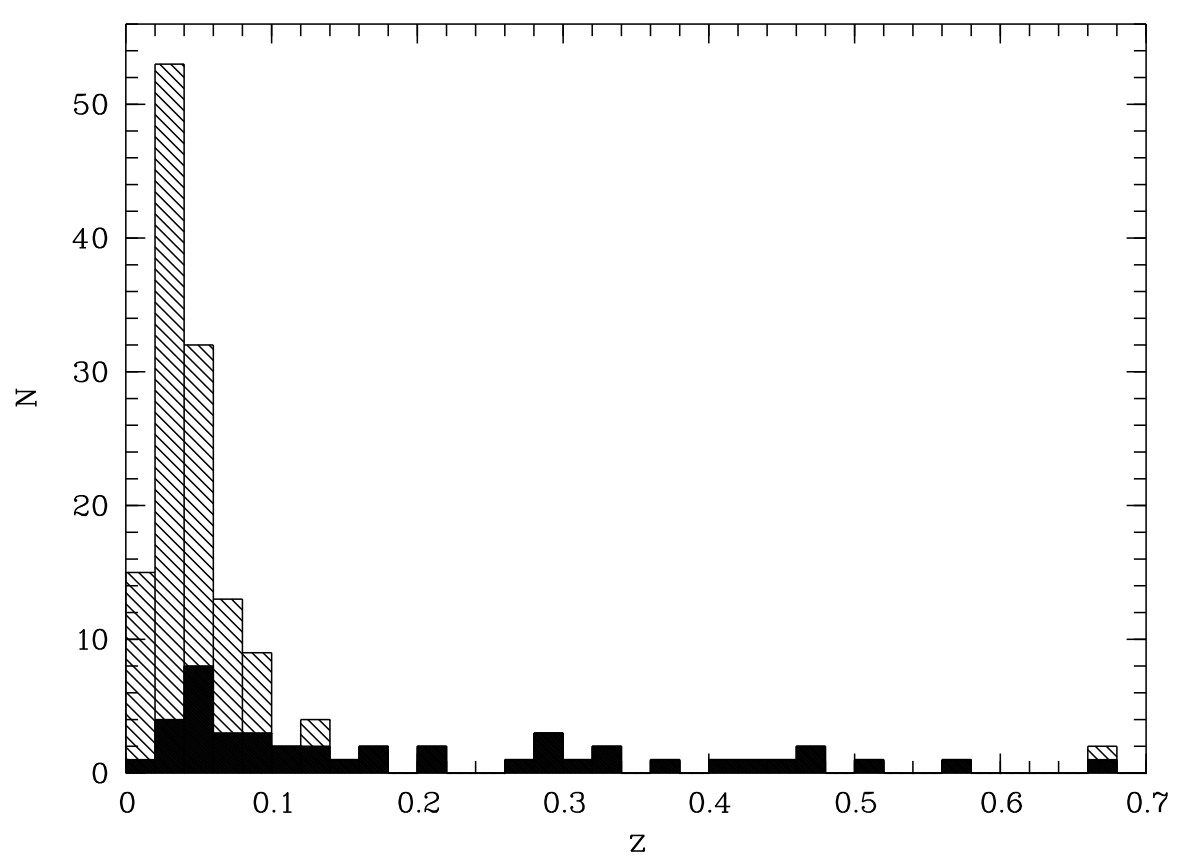

Abbildung 3.6: Häufigkeitsverteilung der Rotverschiebungen der Quellen. Die schraffierten Bereiche der Balken repräsentieren die XGAL, die schwarzen Bereiche die VBLG. Die entsprechenden statistischen Daten sind in Tabelle 3.4 aufgelistet. Während die XGAL sich sehr stark bei kleinen Rotverschiebungen $<0.1$ konzentrieren, zeigen die VBLG eine sehr viel breitere Verteilung mit einer höheren mittleren Rotverschiebung.

Für diejenigen Galaxien, für die sowohl eine Rotverschiebung aus dem Emissionslinienspektrum wie auch aus dem Absorptionslinienspektrum bestimmt werden konnte, ergab sich in keinem Fall eine signifikante Abweichung zwischen beiden Meßwerten.

Für die BL Lac Objekte konnte aufgrund ihrer linienlosen Spektren naturgemäß keine Rotverschiebung bestimmt werden.

Abbildung 3.6 zeigt die Häufigkeitsverteilung aller gemessenen Rotverschiebungen. Während sich die XGAL (schraffierte Bereiche der Balken) sehr stark bei kleinen Rotverschiebungen $z<0.1$ konzentrieren, zeigen die VBLG (schwarze Balken) eine sehr viel breitere Verteilung mit einer höheren mittleren Rotverschiebung.

Dieses Verhalten spiegelt sich auch in den in Tabelle 3.4 aufgelisteten statistischen Daten wider: sowohl der Mittelwert als auch die Streuung der Rotverschiebung sind für die XGAL deutlich geringer als für die VBLG.

Die Ursache dafür liegt in der Selektion der beiden Samples. Aufgrund der Selektionskriterien der XGAL (Detektion im ROSAT All-Sky Survey; optisches, extragalaktisches 
Tabelle 3.4: Statistik der Rotverschiebungen der Quellen für das Gesamtsample sowie die Untergruppen VBLG, XGAL, XAGN und XAGN-1. Aufgelistet sind jeweils die Größe des Samples N, Minimum und Maximum sowie Mittelwert $<\mathrm{z}>$ und Standardabweichung $\sigma$. In ähnlicher Weise werden auch in den folgenden Kapiteln statistischen Daten aufgelistet.

\begin{tabular}{lrcccc}
\hline \hline & & \multicolumn{4}{c}{$\mathrm{z}$} \\
& $\mathrm{N}$ & Min. & Max. & $<\mathrm{z}>$ & $\sigma$ \\
\hline Gesamtsample & 150 & 0.009 & 0.678 & 0.092 & 0.129 \\
VBLG & 44 & 0.009 & 0.669 & 0.201 & 0.174 \\
XGAL & 106 & 0.013 & 0.678 & 0.047 & 0.066 \\
XAGN & 83 & 0.013 & 0.678 & 0.048 & 0.071 \\
XAGN-1 & 57 & 0.013 & 0.678 & 0.054 & 0.088 \\
\hline \hline
\end{tabular}

Gegenstück) befinden sich diese alle in geringer Entfernung. Die VBLG hingegen sind recht selten und im Mittel leuchtkräftiger als die XGAL und wurden daher in einem sehr viel größeren Raumbereich des Universums detektiert. Dieser Unterschied wird sich in den folgenden Abschnitten wiederfinden.

Die Unterschiede zwischen den Gruppen XGAL, XAGN und XAGN-1 sind gering und angesichts der Streuung nicht signifikant. In der Tendenz ist die mittlere Rotverschiebung in der obigen Sequenz leicht zunehmend.

\subsection{Räumliche Verteilung der XGAL}

In Abbildung 3.7 ist die räumliche Verteilung der röntgenselektierten Galaxien in AitoffProjektion dargestellt. Es werden sowohl alle ursprünglich selektierten Objekte des Samples als Kreuze wie auch die letztendlich erfolgreich spektroskopierten Galaxien (offene Symbole) gezeigt.

Nachdem durch die optische Spektroskopie festgestellt wurde, dass es sich bei den röntgenselektierten Galaxien tatsächlich überwiegend um AGN handelt, stellt sich sofort die bereits oben kurz angesprochene Frage (s. Abschnitt 2.2), warum diese Quellen zuvor nicht als AGN bekannt waren.

Wären die Quellen statistisch zufällig früheren Suchprogrammen für Aktive Galaxien entgangen, so könnte man vermuten, dass auf der Südhemisphäre mehr neue röntgenselektierte AGN gefunden werden sollten, denn klassische AGN-Suchprogramme wie 


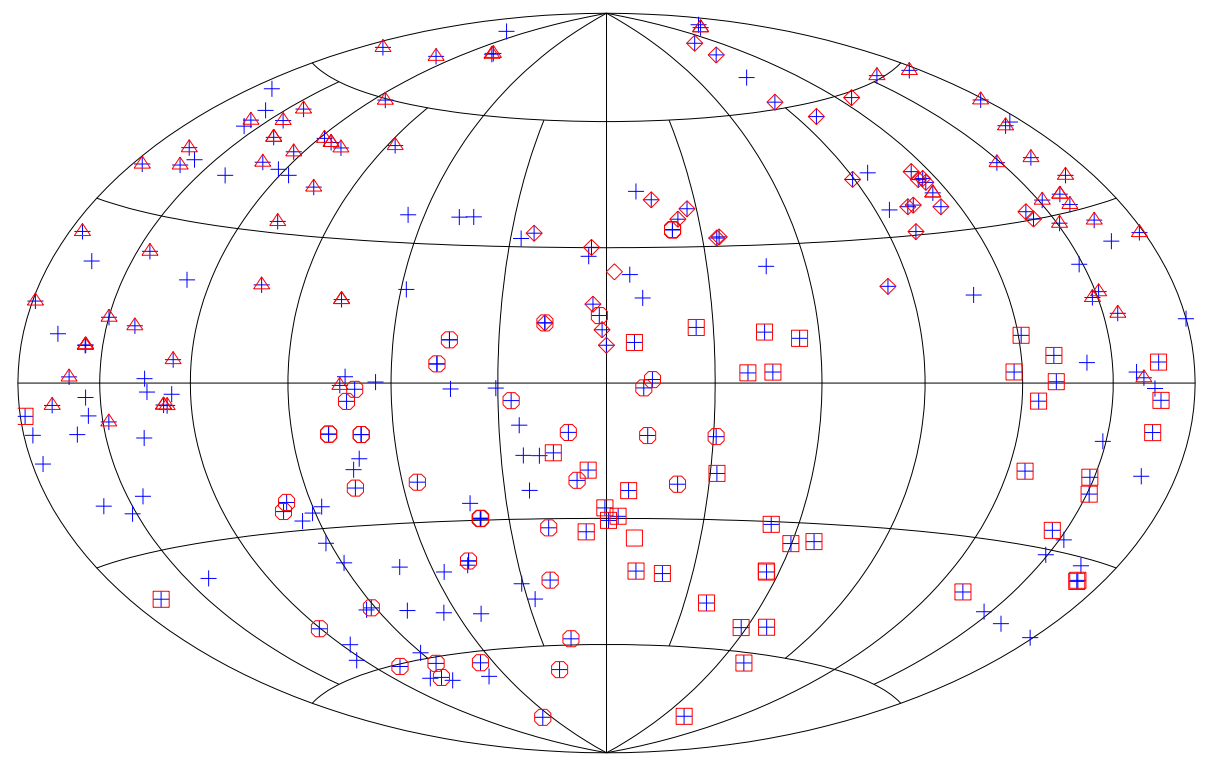

\begin{abstract}
Abbildung 3.7: Räumliche Verteilung der Quellen des Samples röntgenselektierter Galaxien (Aitoff-Projektion). Die Kreuze bezeichnen alle ursprünglich selektierten Objekte des Samples, die anderen Symbole diejenigen Objekte, von denen Spektren hinreichender Qualität gewonnen werden konnten. Außer den durch die galaktische Scheibe bedingten Freiräumen sind keine besonderen Unregelmäßigkeiten in der Verteilung auffällig.
\end{abstract}

z.B. die Objektivprismendurchmusterungen von Markarian (1967) (für eine vollständige Liste der insgesamt 1500 Markarian-Galaxien siehe Mazzarella \& Balzano 1986) konzentrierten sich auf den Nordhimmel.

Für einen solchen Effekt liefern unsere in Abbildung 3.7 dargestellten Daten keine Hinweise. Größere Lücken sind auf die galaktische Scheibe zurückzuführen; die Beobachtung extragalaktischer Quellen bei geringer galaktischer Breite wird durch Absorption verhindert. Ansonsten ist eine Konzentration der Quellen auf die Südhemisphäre nicht $\mathrm{zu}$ erkennen und damit aus der räumlichen Verteilung der XGAL kein weiterer Hinweis auf ihre Natur zu entnehmen. 


\section{Kapitel 4}

\section{Der Multifrequenzdatensatz}

In diesem Kapitel werden die Kontinuumsdatensätze der vier Frequenzbereiche Optisch, Röntgen, Radio und Ferninfrarot vorgestellt. Es werden sowohl die Quellen, denen die Daten entnommen sind, als auch ihre generellen Eigenschaften besprochen. Zuvor wird die weiter unten angewandte Korrelationsanalyse sowie das der Berechnung der Leuchtkräfte zu Grunde liegende kosmologische Modell skizziert.

\subsection{Korrelationsanalyse}

Der lineare, Pearson'sche Korrelationskoeffizient $r_{p}$ ist ein Indikator dafür, wie gut Messpunkte $\left(x_{i}, y_{i}\right)$ zu einer Geraden passen. Er ist durch

$$
r_{p}=\frac{\Sigma\left(x_{i}-<x>\right)\left(y_{i}-<y>\right)}{\sqrt{\Sigma\left(x_{i}-<x>\right)^{2} \Sigma\left(y_{i}-<y>\right)^{2}}}
$$

definiert, wobei $\langle x\rangle$ und $\langle y\rangle$ die jeweiligen arithmetischen Mittelwerte sind. Dabei wird vorausgesetzt, dass die betrachteten Größen normalverteilt sind.

Diese Voraussetzung ist nicht erforderlich zur Berechnung der Rangkorrelation nach Spearman $r_{s}$, die sich daher auch anbietet, wenn die Verteilungsfunktion der Messwerte nicht genau bekannt ist. Aus diesem Grunde wird im Folgenden immer die SpearmanKorrelation mit

$$
r_{s}=1-\frac{6 \Sigma D_{i}^{2}}{N\left(N^{2}-1\right)}
$$

betrachtet. Dabei ist $\mathrm{N}$ die Anzahl der Messwertpaare und $D_{i}$ die Differenz in den Rangplätzen der beiden zusammengehörigen Werte eines Wertepaares. 
Ein weiterer Vorteil der Spearman-Korrelation besteht darin, dass das Ergebnis nicht davon abhängt, ob eine Größe $x$ oder eine monotone Funktion von $x$ (z.B. $\log x$ ) analysiert wird. Das ist daher von Bedeutung, da die meisten Größen hier logarithmisch betrachtet und graphisch dargestellt werden.

Zusätzlich zum Korrelationskoeffizienten $r$ wird im Folgenden noch die Wahrscheinlichkeit $P\left(r \geq r_{0}\right)$ der sog. Null-Hypothese angegeben, die besagt, dass $\mathrm{N}$ Messungen zweier unkorrelierter Variablen einen Korrelationskoeffizienten $\mathrm{r}$ größer oder gleich $r_{0}$ liefern. Ist $\mathrm{P}$ kleiner als 5\%, wird häufig angenommen, dass eine signifikante Korrelation vorliegt; bei $P<1 \%$ kann man von einer hochsignifikanten Korrelation ausgehen.

\subsection{Kosmologisches Modell}

Ein erheblicher Teil der Galaxien dieses Samples befindet sich in kosmologischen Entfernungen; ab Rotverschiebungen von etwa $z>0.1$ haben kosmologische Effekte gegenüber der euklidischen Geometrie signifikante Auswirkungen bei der Berechnung von Entfernungen und Leuchtkräften. Im Folgenden wird für alle Berechnungen die Einstein-de Sitter-Kosmologie $\left(q_{0}=0.5\right)$ benutzt.

Beobachtet man für eine Quelle in einem bestimmten Frequenzband einen Strahlungsfluss $f$, also die Energie, die pro Zeit- und Flächeneinheit den Detektor trifft, so berechnet sich die Leuchtkraft $L$ der Quelle nach

$$
L=4 \pi r_{L}^{2} \cdot f
$$

Dabei ist $r_{L}$ die so genannte Leuchtkraftentfernung. Im nahen Kosmos $(z \ll 0.1)$ entspricht $r_{L}$ dem geometrischen Abstand $r$. Allgemein ist die Leuchtkraftentfernung derjenige Abstand, für den auch auf kosmologischen Skalen die im nahen Kosmos gültige Gleichung 4.3 gilt. Sie ist mit dem geometrischen Abstand durch

$$
r_{L}=r \cdot(z+1)
$$

verknüpft.

Die Leuchtkraftentfernung $r_{L}$ eines Objektes ist von der zu Grunde liegenden Kosmologie abhängig. Mit der Lichtgeschwindigkeit $c$ gilt die (verallgemeinerte) HubbleBeziehung

$$
r_{L}=\frac{c z}{H_{0}} \cdot \Psi\left(q_{0}, z\right)
$$

mit 


$$
\Psi\left(q_{0}, z\right)=\frac{1}{q_{0}}+\frac{q_{0}-1}{q_{0}^{2} z}\left(\sqrt{2 q_{0} z+1}-1\right)
$$

Für den nahen Kosmos lässt sich $\Psi\left(q_{0}, z\right) \approx 1$ approximieren und man erhält als Näherung die klassische Hubble-Beziehung.

Die Hubble-Konstante $H_{0}$ beschreibt die derzeitige Expansionsgeschwindigkeit des Universums und der Dezelerations- oder Verzögerungsparameter $q_{0}$ deren zeitliche Entwicklung. Im Folgenden werden eine Hubble-Konstante von $H_{0}=75 \mathrm{~km} \mathrm{~s}^{-1} \mathrm{Mpc}^{-1}$ und ein Verzögerungsparameter von $q_{0}=0.5$ (Einstein-de Sitter-Kosmos) angenommen.

Analog zur Behandlung der Leuchtkräfte muss auch bei der Umrechnung von scheinbaren in absolute bolometrische Magnituden für kosmologische Entfernungen statt der geometrischen Entfernung die Leuchtkraftentfernung benutzt werden:

$$
m-M=5 \log r_{L}[p c]-5,
$$

wobei $r_{L}$ definitionsgemäß in parsec anzugeben ist; die absolute Helligkeit eines Objektes ist diejenige, die es scheinbar hätte, wenn es sich in einer Entfernung von $r_{L}=10 \mathrm{pc}$ befände. In dieser Entfernung ist das so genannte Entfernungsmodul $m-M=0$.

\subsection{Optische Helligkeiten}

Im Gegensatz zu den anderen hier diskutierten Spektralbereichen steht für den optischen Bereich außer Frage, dass die Objekte detektiert werden konnten. Es sind nur Galaxien in das Sample aufgenommen worden, für die ein optisches Gesamtspektrum hinreichender Qualität gewonnen werden konnte (s. Kapitel 3). Insofern muss die optische Helligkeit für alle Quellen als zumindest indirektes Selektionskriterium betrachtet werden, auch wenn ein explizites Beobachtungslimit nicht konkret angegeben werden kann.

\subsubsection{Optische Galaxienkataloge}

Die optischen Helligkeiten der Galaxien wurden aus der Literatur entnommen. Primäre Quelle dafür war der „Catalogue of Principal Galaxies“ PGC (Paturel et al. 1989). Die genaue Bestimmung der optischen Helligkeiten in einem aufwändigen Verfahren ist dort ausführlich beschrieben. Wie in Abschnitt 2.2 erläutert wurde dieser Katalog bereits zur Selektion des XGAL-Samples verwendet, da er der umfassendste, zu diesem Zweck geeignete Galaxien-Katalog ist. 
Der PGC umfasst 73197 Galaxien und damit insbesondere auch alle gängigen früheren Galaxienkataloge, wie z.B. NGC, IC, UGC, ESO, MCG und CGCG. Die angegebenen Positionen der Objekte sind in den meisten Fällen genauer als $10^{\prime \prime}$. Der PGC enthält scheinbare totale B-Helligkeiten $\mathrm{B}_{T}$ aller Objekte, für die entsprechende Messungen zur Verfügung standen. Dabei wurde viel Wert auf eine möglichst große Homogenität dieser Helligkeiten gelegt; gegebenenfalls wurden die Daten aus den verschiedenen Quellen entsprechend korrigiert. Daher eignen sich die PGC-Helligkeiten für dieses Projekt besonders gut.

Falls im PGC keine B-Helligkeiten angegeben waren, wurden diese der NED entnommen. In dieser Datenbank sind insbesondere auch neuere Messungen enthalten, die im PGC noch nicht berücksichtigt werden konnten. Dabei wurden die gleichen Kriterien zu Grunde gelegt, d.h. es wurden möglichst totale B-Helligkeiten verwendet. In einigen wenigen Fällen musste auf andere optische Helligkeiten zurückgegriffen werden, oder es wurden Helligkeiten von Begleitobjekten anhand der Aquisitionsaufnahmen im Vergleich mit der Primärquelle geschätzt.

\subsubsection{Der optische Datensatz}

Mit Hilfe von Gleichung (4.7) wurden aus den scheinbaren $\left(\mathrm{m}_{\mathrm{B}}\right)$ die absoluten optischen Helligkeiten $\mathrm{M}_{\mathrm{B}}$ berechnet. Abbildung 4.1 zeigt für jeden Galaxien-Typ den Anteil von Objekten, für den auf diese Weise Absoluthelligkeiten berechnet werden konnten (schraffiert). Wie man sieht, war dies für fast das gesamte Sample möglich. Die einzige Ausnahme bilden die BL Lac Objekte: da ihre Entfernungen nicht bekannt sind, konnten auch keine Absoluthelligkeiten berechnet werden.

In Abbildung 4.2 ist die Häufigkeitsverteilung der optischen Absoluthelligkeiten aufgetragen. Dabei sind die VBLG (schwarz) und die XGAL (schraffiert) dargestellt. Insgesamt erstrecken sich die Helligkeiten über etwa zehn Magnituden. Während die Verteilung der VBLG relativ flach ist, konzentrieren sich die XGAL sehr stark im leuchtschwächerem Bereich. Nur einzelne XGAL sind absolut heller als -23 mag.

Diese Eigenschaften spiegeln sich in den in Tabelle 4.1 aufgelisteten statistischen Daten der optischen Absoluthelligkeiten wider: während die VBLG im Mittel um genau zwei Magnituden heller als die XGAL sind, ist die korrespondierende Standardabweichung fast doppelt so hoch.

Um im Folgenden einen exakteren Vergleich zu ermöglichen, sind für die röntgenselektierten Galaxien die drei Untergruppen XGAL, XAGN und XAGN-1 einzeln aufgeführt. Wie Tabelle 4.1 zeigt, sind sie hinsichtlich der statistischen Daten von $\mathrm{M}_{\mathrm{B}}$ nahezu identisch.

In Abbildung 4.3 sind die optischen Absoluthelligkeiten gegen die Rotverschiebung aufgetragen. Die absolut helleren Objekte werden bei höheren Rotverschiebungen 


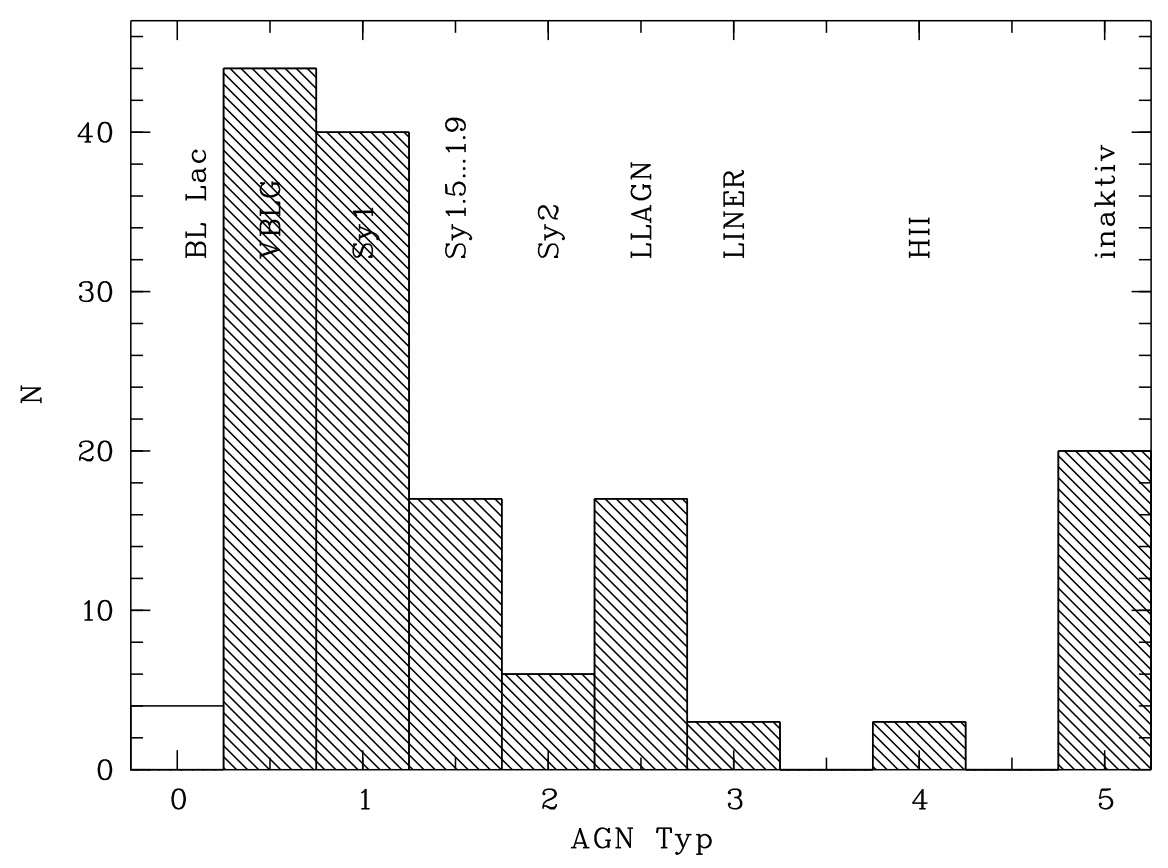

Abbildung 4.1: Schraffiert dargestellt ist der Anteil der verschiedenen GalaxienTypen am Gesamtsample, für die absolute optische Helligkeiten vorliegen. Für die BL Lac Objekte ist die Entfernung und damit auch die Absoluthelligkeit nicht bekannt, für alle anderen Quellen konnten Absoluthelligkeiten berechnet werden.

Tabelle 4.1: Statistik der absoluten optischen Helligkeiten $\mathrm{M}_{\mathrm{B}}$ : aufgelistet sind für das Gesamtsample, die VBLG sowie die Untergruppen XGAL, XAGN und XAGN-1 die Anzahl der Objekte mit absoluten optischen Helligkeiten N sowie Minimum, Maximum, Mittelwert und Standardabweichung von $\mathrm{M}_{\mathrm{B}}$. Die drei letztgenannten Untergruppen sind hinsichtlich ihrer statistischen Daten nahezu identisch.

\begin{tabular}{lrcccc}
\hline \hline & & \multicolumn{4}{c}{$\mathrm{M}_{\mathrm{B}}[\mathrm{mag}]$} \\
& $\mathrm{N}$ & Min. & Max. & $<\mathrm{M}_{\mathrm{B}}>$ & $\sigma$ \\
\hline Gesamtsample & 150 & -27.2 & -17.9 & -21.4 & 1.7 \\
VBLG & 44 & -27.2 & -19.3 & -22.9 & 2.0 \\
XGAL & 106 & -24.5 & -17.9 & -20.7 & 1.1 \\
XAGN & 83 & -24.5 & -17.9 & -20.7 & 1.1 \\
XAGN-1 & 57 & -24.5 & -17.9 & -20.6 & 1.1 \\
\hline \hline
\end{tabular}




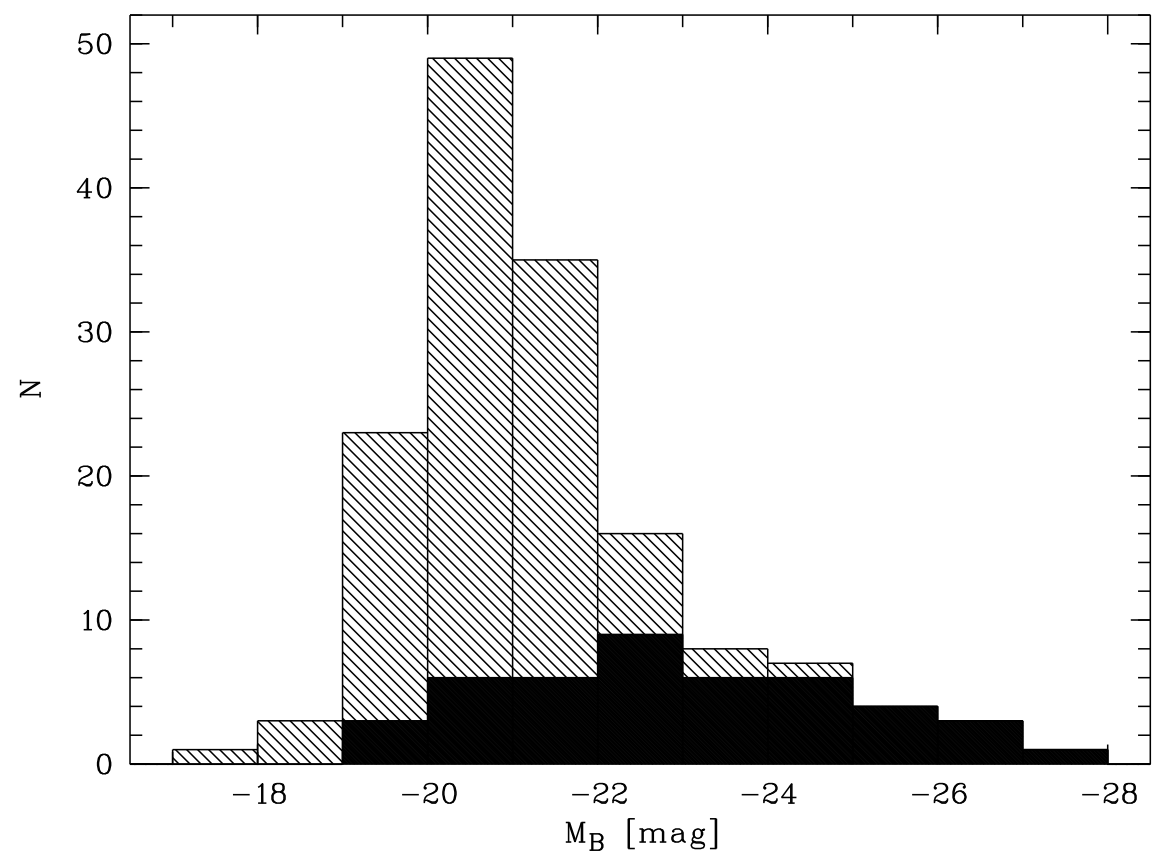

Abbildung 4.2: Dargestellt ist die Häufigkeitsverteilung der absoluten optischen Helligkeiten des Samples, wobei die VBLG schwarz und die XGAL schraffiert gezeichnet sind. Auffällig ist der Gegensatz der flachen und breiten Verteilung der VBLG zu der stark im leuchtschwächeren Bereich konzentrierten Verteilung der XGAL.

beobachtet, alle Quellen liegen in einem in einem relativ engen Band. Dieser Effekt ist fundamentaler Natur und wird auch in den weiteren Frequenzbereichen beobachtet. Er soll daher am Ende dieses Kapitels in Abschnitt 4.7 im Zusammenhang für alle Frequenzbereiche ausführlicher diskutiert werden.

Die absolut hellen Quellen bei hohen Rotverschiebungen sind nahezu ausschließlich VBLG - auch das ist in den anderen Frequenzbereichen ähnlich.

Die Limitierung der optischen Beobachtungen war dadurch gegeben, dass mit den verwendeten Teleskopen der 2m-Klasse ein analysierbares Spektrum gewonnen werden konnte. Da es sich hierbei jedoch um kein „scharfes“ Kriterium handelt, wurde auf die Abschätzung einer Nachweisgrenze verzichtet. Stattdessen wurden zum Vergleich zusätzlich die AGN des Veron-Kataloges (Véron-Cetty \& Véron 2001) als Punkte eingezeichnet. Dieser Katalog ist der umfassendste und enthält fast alle bekannten AGN.

Die Quellen unseres Samples befinden sich zwar eindeutig im am stärksten bevölkerten Gebiet des Diagramms, tendieren aber zum oberen Rand der AGN-Verteilung. Das gilt für die VBLG im noch stärkerem Maße als für die XAGN und bedeutet, dass diese Objekte im Optischen überdurchschnittlich leuchtkräftig sind. 


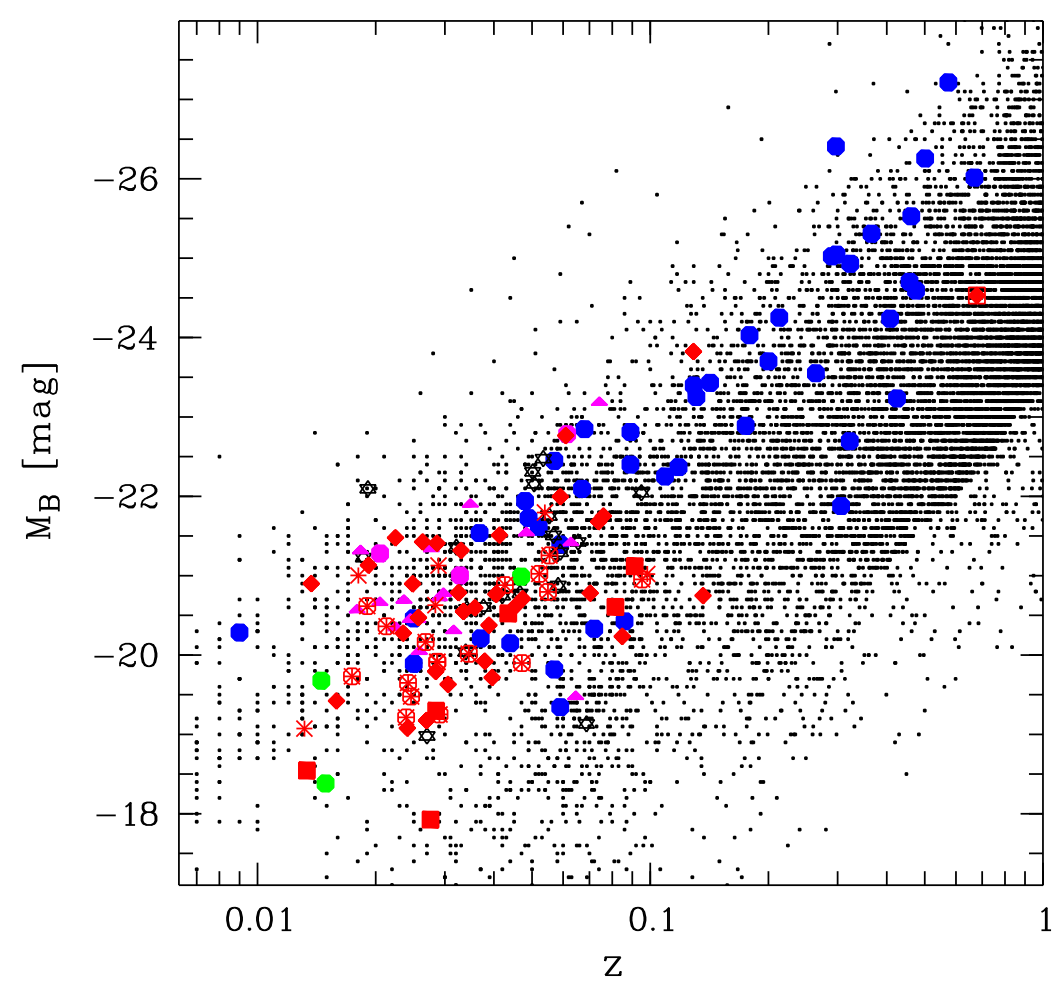

Abbildung 4.3: Optische Absoluthelligkeit als Funktion der Rotverschiebung. Zum Vergleich sind zusätzlich die entsprechenden Werte aller AGN/QSO des Veron-Kataloges (Véron-Cetty \& Véron 2001) als Punkte eingezeichnet. Die absolut helleren Quellen werden bei höheren Rotverschiebungen beobachtet. Wie auch in allen entsprechenden nachfolgenden Graphiken werden die verschiedenen AGN-Typen durch Symbole wie in Abbildung 3.2 erklärt kodiert. Hinzu kommen die durch volle blaue Kreise symbolisierten VBLG.

\subsection{Der Röntgenbereich}

\subsubsection{Röntgen-Kataloge}

Eine der wesentlichen Grundlagen dieser Arbeit sind Röntgendaten des ROSAT-Satelliten, insbesondere die vollständige Himmelsdurchmusterung ROSAT All-Sky Survey RASS. Wie in Kapitel 2.2 ausführlich beschrieben, entstammen die Quellen des XAGNTeilsamples dem ROSAT All-Sky Survey Bright Source Catalouge 1RXS/RASS-BSC, der 18811 Röntgenquellen enthält (Voges et al. 1996, 1999). Die dort aufgelisteten Röntgenzählraten wurden in dieser Arbeit verwendet.

Als Quelle für ROSAT-Daten für das VBLG-Teilsample standen darüber hinaus zwei 
weitere Kataloge zur Verfügung, die auf dedizierten ROSAT-Beobachtungen beruhen. Der „First ROSAT Source Catalog of Pointed Observations with the PSPC“ ROSATSRC/1RXP (Voges et al. 1994) enthält in der hier benutzten Version 74301 Röntgenquellen, die bei 3927 Einzelbeobachtungen mit dem ROSAT-PSPC aufgenommen wurden. Der „First ROSAT Source Catalog of Pointed Observations with the High Resolution Imager" ROSHRI/1RXH ist eine entsprechende Liste von Röntgenquellen, die bei gezielten Beobachtungen mit den HRI registriert wurden (ROSAT Consortium 1999). Sie enthält in der hier benutzten Version (19980601) 59911 Einträge.

Der ROSHRI überdeckt aufgrund des relativ kleinen Gesichtsfeldes des HRI nur einen sehr geringen Teil des Himmels. Außerdem sind durch Verwendung des StandardReduktionsverfahrens SASS in Verbindung mit der relativ hohen räumlichen Auflösung des Instrumentes gelegentlich stärkere Röntgenquellen fälschlicherweise als mehrere Einzelquellen registriert. Ein Vergleich von HRI- und PSPC-Daten ist nur eingeschränkt möglich.

Aus dem vorliegenden VBLG-Sample sind nur sehr wenig Objekte im ROSHRI aufgeführt; diese sind alle ebenfalls in mindestens einem der beiden anderen verwendeten Kataloge enthalten. Auf Grund der geschilderten Schwierigkeiten wurde daher auf die Verwendung der wenigen vorhandenen ROSHRI-Daten ganz verzichtet.

Im RASS-Katalog 1RXS sind 34 und im ROSATSRC 23 der VBLG aufgeführt; davon sind wiederum 15 in beiden Katalogen enthalten. Bei letzteren sind die entsprechenden Zählraten i.d.R. von gleicher Größenordnung. Um eine möglichst große Homogenität der Daten zu erzielen, wurden in diesen Fällen immer die Daten aus dem 1RXS benutzt.

Der Survey RASS und die pointierten PSPC-Beobachtungen sind mit zwei verschiedenen, aber nahezu baugleichen PSP-Instrumenten gemacht worden (B bzw. C). Die Unterschiede zwischen den Instrumenten sind sehr gering und für diese Untersuchung völlig unerheblich.

\subsubsection{Galaxiengruppen}

ESO 290-3 ist ein Gruppe aus drei Galaxien. Welche der drei die Quelle der Röntgenemission ist, konnte auch anhand der Röntgenkonturen nicht entschieden werden. Optische Spektren wurden von der nordwestlichen (NW) und der nordöstlichen (NE) Galaxie aufgenommen, nicht jedoch von der südlichen (S). Aktivität konnte bei beiden spektroskopierten Objekten nicht nachgewiesen werden. Da nach den Röntgenkonturen die Galaxie NE mit etwas größerer Wahrscheinlichkeit als die Galaxie NW die Röntgenquelle ist, wird sie im Sample geführt (ESO 290-3 NE). Es kann aber nicht ausgeschlossen werden, dass die Galaxie S die eigentliche Röntgenquelle ist.

ESO 407-17 ist ein Galaxienpaar. Die im Optischen deutlich hellere Hauptkomponente erwies sich als aktive NLS1 Galaxie; daher wurde die schwächere Komponente nicht 
mehr spektroskopiert. Relativ starke Gezeitenarme sind deutliche Anzeichen für Wechselwirkung und legen nahe, dass es sich um ein echtes, wechselwirkendes Galaxienpaar handelt. Die Rotverschiebung der schwächeren Komponente ist nicht bekannt.

In unmittelbarer Nähe des Galaxienpaares AM 2354-304 befindet sich mit UGC 12890 eine weitere Galaxie. Hinsichtlich ihrer Positionen könnten alle drei Galaxien Quelle der Röntgenstrahlung sein, UGC 12890 allerdings mit deutlich geringerer Wahrscheinlichkeit. Nach den Röntgenkonturen ist die östliche Komponente AM 2354-304 E die wahrscheinlichste Quelle der Röntgenstrahlung. Die optische Spektroskopie zeigt, dass es sich hierbei um eine Seyfert-1 Galaxie handelt. AM 2354-304 W (westliche Komponente) ist eine Starburst-Galaxie ( $z=0.0299)$, UGC 12890 eine LINER-Galaxie $(z=0.0394)$. Im Sample wird folglich AM 2354-304 E geführt.

MCG +7-37-18 ist ein Galaxienpaar. Die nördlichere Galaxie ist im Optischen etwa eine Magnitude heller, zeigt aber keinerlei Linienemission. Die südlichere ist eine StarburstGalaxie und wurde im Gegensatz zur nördlichen Galaxie im NVSS als Radioquelle detektiert. Anhand der Röntgendaten kann nicht sicher entschieden werden, welche die Röntgenquelle ist: sie deuten aber auf die nördliche Galaxie hin. Um auch der Möglichkeit gerecht zu werden, dass die südliche Galaxie die Röntgenquelle ist, werden beide Galaxien mit der gemessenen Röntgenleuchtkraft aufgeführt.

Im Feld der Galaxie ESO 381-7 befindet sich eine weitere Röntgenquelle ( $\alpha=1240$ $47.9 ; \delta=-334618$ ). Das optische Spektrum zeigt, dass es sich hierbei um ein QSO mit einer Rotverschiebung von $z=0.8805$ handelt. Die Röntgenquelle ist im 1RXH registriert, allerdings nicht eindeutig: es sind vier Einträge verzeichnet. Da es sich außerdem nicht um eine primäre Quelle des Samples handelt, wurde sie nicht weiter berücksichtigt.

\subsubsection{Der Röntgen-Datensatz}

Aus den ROSAT-Zählraten wurden unter Verwendung der EXSAS-Routine MODEL/ SPECTRUM Röntgenflüsse und -leuchtkräfte berechnet. Dazu wurde ein Spektralverlauf in Form eines Potenzgesetzes

$$
f_{E} d E \propto E^{-\Gamma+1} d E
$$

angenommen, wobei $f_{E} d E$ der Energiefluss im Intervall von $E$ bis $E+d E$ ist. Es wurde weiterhin ein fester Photonenindex $\Gamma=2.3$ angenommen, ein typischer Wert für mit ROSAT beobachtete Galaxien (z.B. Hasinger et al. 1991, Walter \& Fink 1993). Die interstellare Absorption wurde nach Dickey \& Lockman (1990) korrigiert, eine mögliche interne Absorption in den Galaxien wurde nicht korrigiert. 


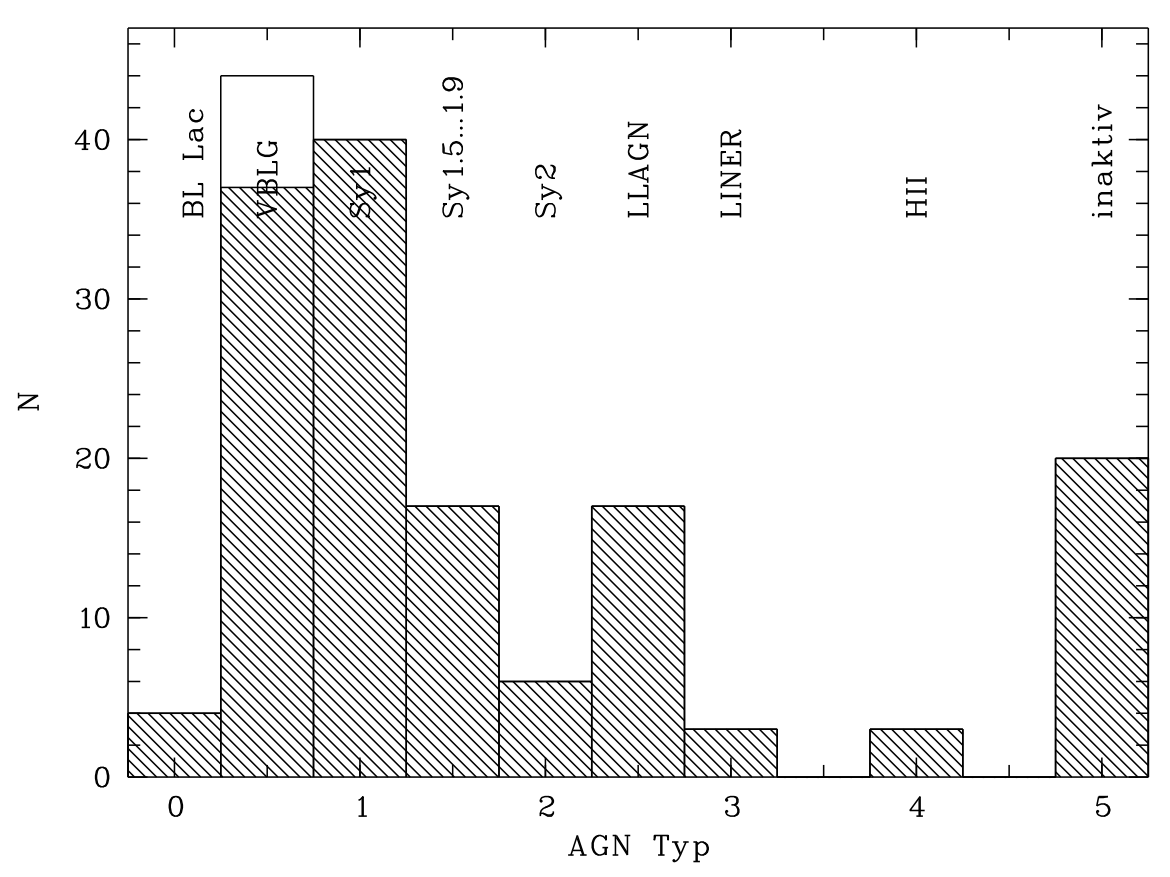

Abbildung 4.4: Schraffiert dargestellt ist der Anteil des Samples, für den ROSATRöntgenflüsse vorliegen. Für sieben Objekte aus dem VBLG-Teilsample ist dies nicht der Fall - diese sind durch den nicht schraffierten Bereich gekennzeichnet. Aufgrund der Auswahl des Samples ist der Röntgendatensatz für das röntgenselektierte XGAL-Teilsample vollständig.

In Abbildung 4.4 ist schraffiert dargestellt, für welchen Teil des Samples ROSATRöntgenflüsse vorliegen. Aufgrund der Selektion des Samples ist das für das XGALTeilsample zu 100\% der Fall. Bei den VBLG sind nur für sieben Objekte keine Röntgenflüsse vorhanden.

Die Häufigkeitsverteilung der Röntgenleuchtkräfte ist in Abbildung 4.5 dargestellt. Das VBLG-Teilsample wurde dabei wiederum schwarz gezeichnet. Das obere Ende der Leuchtkraftverteilung wird im Wesentlichen durch die VBLG gebildet, die sich allerdings auch bis zum leuchtschwachen Ende erstrecken. Im Gegensatz dazu zeigen die XGAL eine sehr ausgeprägte Konzentration um $\mathrm{L}_{X}=10^{43} \mathrm{erg} \mathrm{s}^{-1}$.

In Tabelle 4.2 sind die statistischen Parameter des Röntgendatensatzes aufgelistet. Im Mittel liegt die Röntgenleuchtkraft der VBLG um gut eine Größenordnung über derjenigen der XAGN. Die sehr viel breitere Verteilung der VBLG im Vergleich zu den XAGN zeigt sich in einer fast doppelt so großen Standardabweichung von $\log \mathrm{L}_{\mathrm{X}}$. Auch hier unterscheiden sich XGAL, XAGN und XAGN-1 nur geringfügig. 


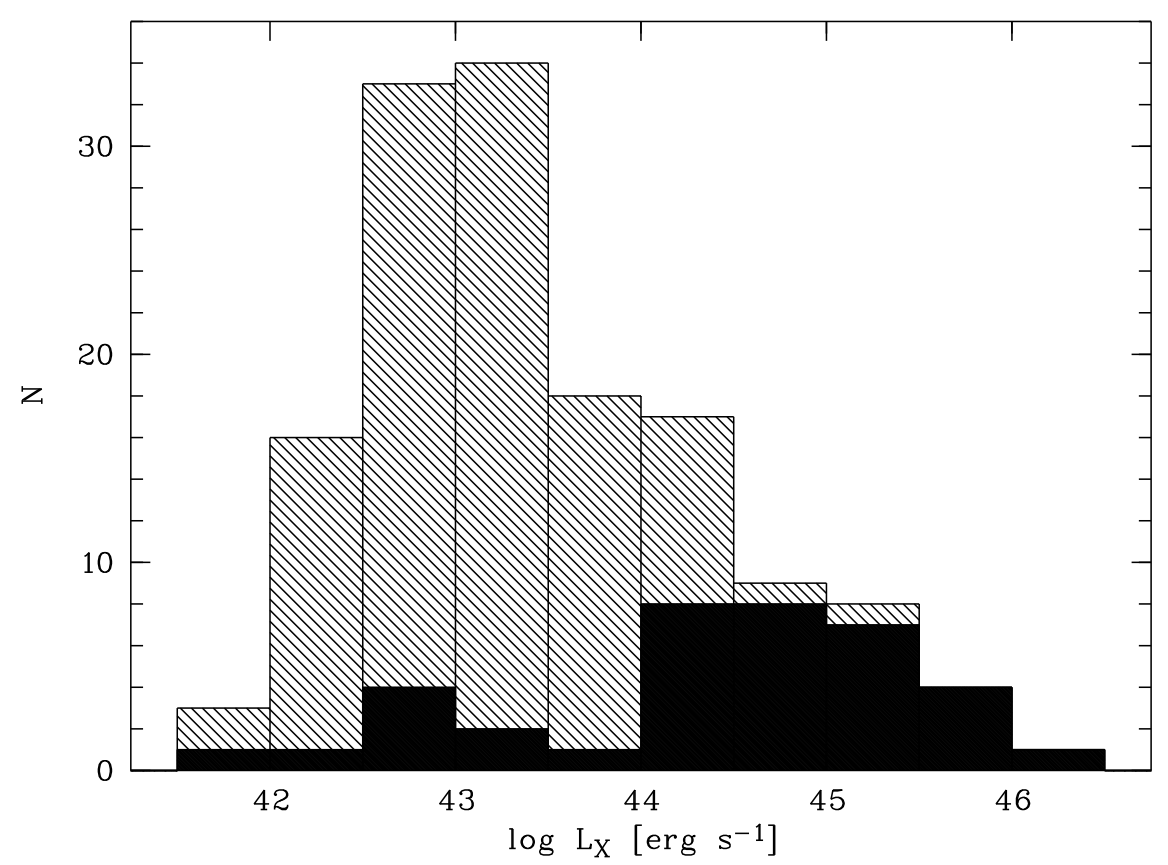

Abbildung 4.5: Häufigkeitsverteilung der Röntgenleuchtkräfte $\log \mathrm{L}_{X}$. Die schwarz dargestellten VBLG dominieren deutlich das obere Ende der Leuchtkraftverteilung, während sich die XGAL (schraffiert) stark im mittleren Bereich um $\mathrm{L}_{X}=10^{43} \mathrm{erg} \mathrm{s}^{-1}$ konzentrieren.

Tabelle 4.2: Statistik der Röntgenleuchtkräfte: aufgelistet sind für das Gesamtsample und die Untergruppen VBLG sowie XGAL, XAGN und XAGN-1 die Anzahl der Objekte mit Röntgen-Leuchtkräften N sowie Minimum, Maximum, Mittelwert und Standardabweichung von $\log \mathrm{L}_{\mathrm{X}}$.

\begin{tabular}{lccccc}
\hline \hline & & \multicolumn{4}{c}{$\log \mathrm{L}_{X}\left[\mathrm{erg} \mathrm{s}^{-1}\right]$} \\
& $\mathrm{N}$ & Min. & $\operatorname{Max}$. & $<\log \mathrm{L}_{X}>$ & $\sigma$ \\
\hline Gesamtsample & 143 & 41.8 & 46.0 & 43.5 & 0.9 \\
VBLG & 37 & 41.9 & 46.0 & 44.4 & 1.1 \\
XGAL & 106 & 41.8 & 45.0 & 43.1 & 0.6 \\
XAGN & 83 & 41.8 & 45.0 & 43.2 & 0.6 \\
XAGN-1 & 57 & 41.8 & 45.0 & 43.3 & 0.6 \\
\hline \hline
\end{tabular}




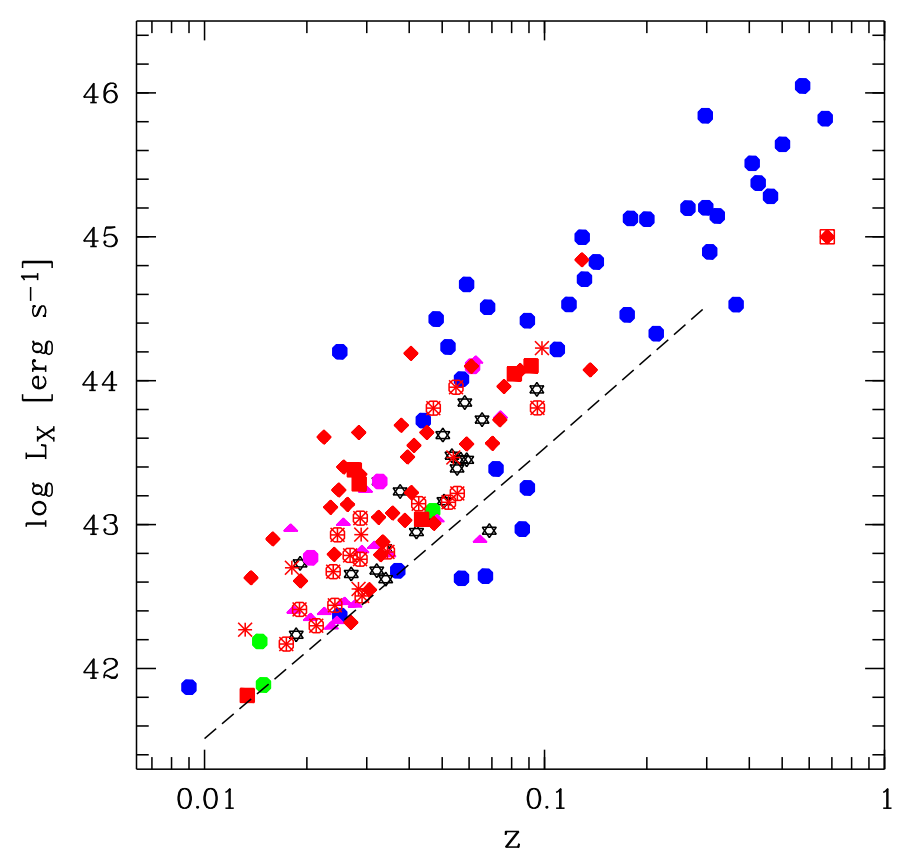

Abbildung 4.6: Röntgenleuchtkraft als Funktion der Rotverschiebung. Die gestichelte Linie entspricht der RASS-Selektionsgrenze von $0.05 \mathrm{cts} / \mathrm{s}$. Während die XGAL aufgrund der Selektion des Samples relativ dicht oberhalb dieser Grenze liegen, ist die Streuung der VBLG deutlich größer.

Abbildung 4.6 zeigt die Röntgenleuchtkräfte als Funktion der Rotverschiebung. Für die RASS-Beobachtungen wurde die Selektionsgrenze in der Zählrate von $0.05 \mathrm{cts} / \mathrm{s}$ in Röntgenleuchtkraft umgerechnet und als gestrichelte Line eingezeichnet. Dabei wurde keine interstellare Absorption angenommen.

Naturgemäß liegen nahezu alle Quellen des XGAL-Samples oberhalb dieser Grenze. Eine Ausnahme bildet VII Zw 608, eine Doppelgalaxie, bei der nicht sicher entschieden werden konnte, ob VII Zw 608 selbst $(z=0.0646)$ oder ihr Begleiter $(z=0.0688)$ die Quelle der Röntgenstrahlung ist. Aufgrund der Röntgenkonturplots wurde angenommen, dass beide Quellen gleichwertig je die Hälfte des Gesamtflusses beisteuern. Somit liegen beide knapp unterhalb der rechnerischen Selektionsgrenze. Die Streuung der VBLG um die Selektionsgrenze ist größer: während einige unterhalb dieser Grenze liegen, befinden sich etliche auch deutlich oberhalb.

Der generelle Anstieg der Leuchtkraft mit der Rotverschiebung entspricht dem Verhalten der absoluten optischen Helligkeit und wird weiter unten (Abschnitt 4.7) noch genauer besprochen. 
Tabelle 4.3: Wesentliche Charakteristika der verwendeten Radiodurchmusterungen bzw. der daraus resultierenden Radiokataloge. Der FIRST-Katalog ist noch nicht fertiggestellt; angegeben sind die Daten der hier benuzten Version. Die in der letzten Spalte angegebene Anzahl der Quellen ist ein gutes Maß für die Ergiebigkeit des jeweiligen Kataloges.

\begin{tabular}{llcccr}
\hline \hline Katalog & Teleskop & $\begin{array}{c}v \\
{[\mathrm{GHz}]}\end{array}$ & $\begin{array}{c}\text { Himmels- } \\
\text { überdeckung }\end{array}$ & $\begin{array}{c}\text { Flusslimit } \\
{[\mathrm{mJy}]}\end{array}$ & $\begin{array}{c}\text { Anzahl } \\
\text { Quellen }\end{array}$ \\
\hline 87GB & Green Bank & 4.85 & $0^{\circ}<\delta<+75^{\circ}$ & $25-40$ & 54579 \\
PMN & Parkes & 4.85 & $\delta<+10^{\circ}$ & $20-72$ & 50814 \\
NVSS & VLA & 1.4 & $\delta>-40^{\circ}$ & 2.5 & 1800000 \\
FIRST & VLA & 1.4 & $4760 \square^{2}$ & 0.5 & 437429 \\
\hline \hline
\end{tabular}

\subsection{Der Radiobereich}

Im Gegensatz zu den anderen Spektralbereichen, bei denen von vornherein jeweils die Daten aus einem Katalog dominierten, standen im Radiobereich mehrere zur Verfügung.

\subsubsection{Radiodurchmusterungen}

Um Radioflüsse der Galaxien zu finden, kamen Kataloge von vier Himmelsdurchmusterungen in Frage: „Green Bank“ (87GB), „Parkes-MIT-NRAO“ (PMN), „NRAO VLA Sky Survey“ (NVSS) und „Faint Images of the Radio Sky“ (FIRST). Da diese Durchmusterungen mit unterschiedlichen Radioteleskopen durchgeführt wurden, überdecken sie jeweils unterschiedliche Himmelsbereiche und haben unterschiedliche Empfindlichkeiten und räumliche Auflösungen. Außerdem sind sie bei zwei unterschiedlichen Frequenzen (1.4 GHz bzw. $4.85 \mathrm{GHz}$ ) gemacht worden. Die wesentlichen Charakteristika der Kataloge sind in Tabelle 4.3 zusammengefasst.

Der entscheidende Unterschied zwischen den älteren Durchmusterungen 87GB und PMN einerseits und den neueren NVSS und FIRST andererseits besteht darin, dass erstere mit einer Einzelantenne (,single dish“), letztere aber mit dem „Very Large Array“ VLA, einer interferometrischen Anordnung von bis zu 27 Radioantennen, durchgeführt wurden. Dadurch sind letztere hinsichtlich der räumlichen Auflösung, aber auch der Empfindlichkeit um mindestens eine Größenordnung besser. Im Folgenden werden die vier Durchmusterungen genauer beschrieben. 
Tabelle 4.4: Zonen der PMN-Radiodurchmusterung. Die Empfindlichkeit des Kataloges (unteres Flusslimit) ist in den vier verschiedenen Zonen jeweils unterschiedlich.

\begin{tabular}{lccl}
\hline \hline Zone & Himmelsüberdeckung & Flusslimit & Referenz \\
\hline Süd & $-87.5^{\circ}<\delta<-37^{\circ}$ & $20-50 \mathrm{mJy}$ & Wright et al. (1994) \\
Zenith & $-37^{\circ}<\delta<-29^{\circ}$ & $72 \mathrm{mJy}$ & Griffith et al. (1994) \\
Tropisch & $-29^{\circ}<\delta<-9.5^{\circ}$ & $42 \mathrm{mJy}$ & Griffith et al. (1995) \\
Äquator & $-9.5^{\circ}<\delta<+10^{\circ}$ & $40 \mathrm{mJy}$ & Wright et al. (1996) \\
\hline \hline
\end{tabular}

\section{Green Bank 87GB}

Die Durchmusterung für den „,87GB Catalog of Radio Sources“ (Gregory \& Condon 1991) wurde mit dem NRAO Green Bank 91m-Teleskop im Oktober 1987 bei einer Frequenz von $4.85 \mathrm{GHz}$ durchgeführt. Die räumliche Auflösung beträgt etwa 3.5. Da es sich um ein Transitinstrument handelte, konnte der Himmelsbereich von $0^{\circ}<\delta<+75^{\circ}$ überdeckt werden. In diesem Bereich wurden von Gregory \& Condon (1991) 54579 Quellen mit einer Flussdichte über 25-40 mJy detektiert und katalogisiert.

Aus derselben Durchmusterung haben auch Becker et al. (1991) einen nahezu identischen Katalog erstellt, der 53522 Quellen umfasst.

\section{Parkes-MIT-NRAO PMN}

Die PMN-Durchmusterung wurde mit dem australischen Parkes 64m-Radioteleskop zwischen Juni und November 1990 ebenfalls bei $4.85 \mathrm{GHz}$ durchgeführt (Griffith \& Wright 1993). Sie ist das südliche Pendant zur Green Bank Nordhimmelsdurchmusterung. Die räumliche Auflösung beträgt etwa 4!2 FWHM. Der Katalog ist in vier verschiedene Himmelszonen aufgeteilt, in denen unterschiedliche Empfindlichkeiten zwischen 20 und 72 mJy erreicht werden. Die entsprechenden Daten der einzelnen Zonen sind in Tabelle 4.4 aufgeführt.

\section{NRAO VLA Sky Survey NVSS}

Die Beobachtungen für den $1.4 \mathrm{GHz}$ „NRAO VLA Sky Survey (NVSS)“1 (Condon et al. 1998) wurden im September 1993 mit dem „Very Large Array“ VLA begonnen und

\footnotetext{
${ }^{1}$ siehe ftp://ftp.mrao.cam.ac.uk/nrao/nvss/catalog
} 
hatten bis Ende 1996 den gesamten Himmel nördlich von $-40^{\circ}$ Deklination überdeckt. Nachbeobachtungen bis Januar 1998 füllten nahezu alle vorherigen Lücken.

Aus den Beobachtungsdaten wurde ein Katalog erstellt, der $99.77 \%$ des überdeckten Himmelsbereichs umfasste. Es wurden Karten mit einer räumliche Auflösung von etwa $45^{\prime \prime}$ benutzt. Der Katalog ist komplett bis zu einem Fluss von 2.5 mJy und enthält 1.8 Millionen Quellen.

\section{Faint Images of the Radio Sky FIRST}

Das Projekt „Faint Images of the Radio Sky at Twenty-Centimeters“2 (Becker et al. 1995) ist als Radio-Äquivalent zum optischen „Palomar Observatoy Sky Survey“ geplant. Dementsprechend sollen mit dem VLA „Radiobilder“ des Himmels bei einer Frequenz von $1.4 \mathrm{GHz}$ bzw. einer Wellenlänge von $20 \mathrm{~cm}$ erzeugt werden.

Das FIRST-Projekt ist noch nicht abgeschlossen. Für diese Arbeit stand ein Katalog zur Verfügung, der auf Beobachtungen zwischen 1993 und 1997 beruhte (White et al. 1997). Dabei wurden zwei Himmelsbereiche beobachtet: ein 4150 Quadratgrad großes Feld am Nordhimmel (382 892 Quellen) und ein 610 Quadratgrad großes Feld am Südhimmel (54537 Quellen). Die typische räumliche Auflösung beträgt $5^{\prime \prime}$ und das untere Flusslimit etwa $0.5 \mathrm{mJy}$.

\subsubsection{Quellen der Radiodaten}

Für alle Objekte wurden anhand ihrer Positionen Radio-Gegenstücke in den vier Katalogen gesucht. Der Suchradius um die optische Position der Quelle wurde der jeweiligen räumlichen Auflösung angepasst.

Bei fast allen Objekten mit einem positiven Suchergebnis gab es nur eine Radioquelle innerhalb des Suchradius. In einigen wenigen Fällen gab es eine zentrale dominante Quelle; weitere Quellen innerhalb des Fehlerradius entsprachen offensichtlich nicht der gesuchten Galaxie. Bei nicht eindeutigen Identifikationen wurden die Radiodaten aus dem entsprechenden Katalog nicht übernommen; es gab hierbei keinen Fall, bei dem nicht mit Hilfe eines der anderen Kataloge doch noch eine sichere Identifikation gelang.

Die Radioflüsse der verschiedenen Kataloge sind nicht direkt miteinander vergleichbar. Einerseits wurden sie bei verschiedenen Frequenzen gemessen, andererseits auch mit unterschiedlichen Instrumenten. Weiterhin können die Quellen auch im Radiobereich intrinsisch variabel sein. Gab es jedoch für eine Galaxie besonders stark abweichende Radioflüsse in verschiedenen Katalogen, wurde die Identifikation nochmals genau überprüft.

\footnotetext{
${ }^{2}$ siehe http://sundog.stsci.edu/
} 
Tabelle 4.5: Die Quellen der Radiodaten: aufgelistet ist sowohl absolut als auch relativ, wieviele Galaxien der Teilsample VBLG und XGAL sowie des Gesamtsamples in den jeweiligen Katalogen gefunden wurden. In der letzten Zeile (,,alle Kataloge") ist angegeben, für wieviele Galaxien überhaupt Radiodaten in mindestens einem der Kataloge gefunden wurde. Diese Zahlen sind nur wenig höher als die entsprechenden NVSS-Werte, da der NVSS der umfassendste Katalog ist und auch fast alle Galaxien enthält, die in einem der anderen Kataloge aufgeführt sind.

\begin{tabular}{lcccccc}
\hline \hline Katalog & \multicolumn{2}{c}{ VBLG } & \multicolumn{2}{c}{ XGAL } & \multicolumn{2}{c}{ Gesamtsample } \\
& abs. & rel. & abs. & rel. & abs. & rel. \\
\hline $87 G B$ & 17 & $39 \%$ & 1 & $1 \%$ & 18 & $12 \%$ \\
PMN & 9 & $20 \%$ & 21 & $19 \%$ & 30 & $19 \%$ \\
NVSS & 39 & $87 \%$ & 48 & $44 \%$ & 87 & $56 \%$ \\
FIRST & 9 & $20 \%$ & 6 & $5 \%$ & 15 & $10 \%$ \\
alle Kataloge & 39 & $87 \%$ & 55 & $10 \%$ & 94 & $61 \%$ \\
\hline \hline
\end{tabular}

Ein besonderer Fall ist die Galaxie 3C390.3. Sie ist die stärkste Radioquelle des gesamten Samples und auch aufgrund ihres optischen Spektrums eine prototypische VBLG. In den neuen hochauflösenden Radiodurchmusterungen können ihre ausgedehnten Radiostrukturen („Radiokeulen“) aufgelöst werden, in den älteren hingegen nicht. Dieses Problem könnte in geringem Umfang auch bei anderen VBLG aufgetreten sein, es gab dafür aber keine konkrete Indizien.

Es gibt etliche Publikationen über Radiobeobachtungen von 3C390.3. Hier wird der von Kuhr et al. (1981) angegebene $1.4 \mathrm{GHz}-$ Fluss von $12.8 \mathrm{Jy}$ verwendet.

Insgesamt war der NVSS die mit Abstand ergiebigste Quelle für Radiodaten zu den Galaxien dieses Samples: er enthielt Radioflüsse von 87 Galaxien. Im PMN fanden sich 30, im 87GB 18 und im FIRST 15 Sample-Galaxien. Alle FIRST- und Gregory-Quellen waren auch im NVSS enthalten. Im PMN fanden sich nur sieben Quellen, die nicht auch im NVSS enthalten waren. Diese Werte sind nochmals unterschieden nach VBLG und XGAL in Tabelle 4.5 zusammengefasst.

Dieses Ergebnis ist nicht überraschend: der NVSS ist aufgrund seiner Himmelsüberdeckung und Empfindlichkeit der umfassendste der vier Kataloge. Der FIRST-Katalog ist zwar etwas empfindlicher, überdeckt aber bislang nur einen kleinen Teil des Himmels. Die beiden älteren Kataloge 87GB und PMN sind deutlich unempfindlicher. Dieses manifestiert sich auch in der höchsten Gesamtzahl von Quellen im NVSS (siehe Tabelle 4.3). 


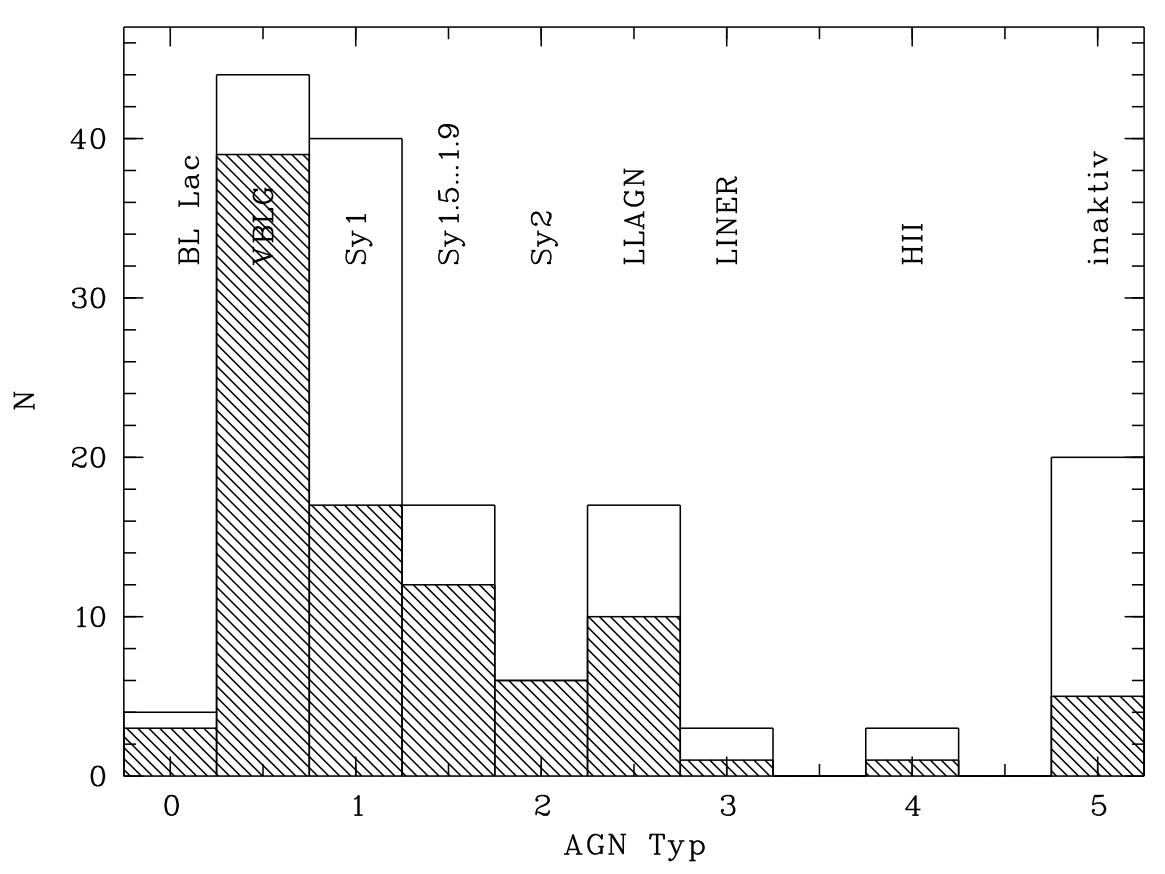

Abbildung 4.7: Der schraffierte Bereich des Histogramms kennzeichnet den Anteil der Objekte des Samples, für den Radiodaten vorliegen. Dieser Anteil ist naturgemäß bei den VBLG besonders hoch, allerdings nicht 100\%. Am geringsten ist er bei den nicht aktiven Galaxien.

Aufgrund der Überschneidungen der vier Kataloge fanden sich von den 94 Quellen, die in mindestens einem der Kataloge sicher identifiziert wurden, 87 Quellen auch im NVSS. Diese Daten werden im Folgenden benutzt. Hinzu kommen PMN-Daten für die restlichen sieben Quellen. Damit wird eine weitgehende Homogenität der Radiodaten erreicht, da 93\% aus demselben Katalog stammen. Gleichzeitig liegen für die Mehrheit der Sample-Galaxien Radiodaten vor.

\subsubsection{Der Radio-Datensatz}

Radiodaten liegen für insgesamt 94 bzw. 61\% der Quellen vor. In Abbildung 4.7 ist für jeden Galaxien-Typ des Samples schraffiert dargestellt, wie groß der Anteil der Objekte mit Radiodaten ist.

Als statistisch nicht signifikant ist anzusehen, dass für alle Seyfert 2-Galaxien Radiodaten vorliegen, da es insgesamt nur sechs Objekte sind. Davon abgesehen ist unter den verschiedenen Galaxien-Typen wie zu erwarten bei den VBLG der Anteil von Quellen mit Radiodaten mit 86\% am höchsten. Allerdings beträgt er nicht 100\% - die Stärke der Radiostrahlung war bei der Selektion des VBLG-Samples kein Auswahlkriterium und 


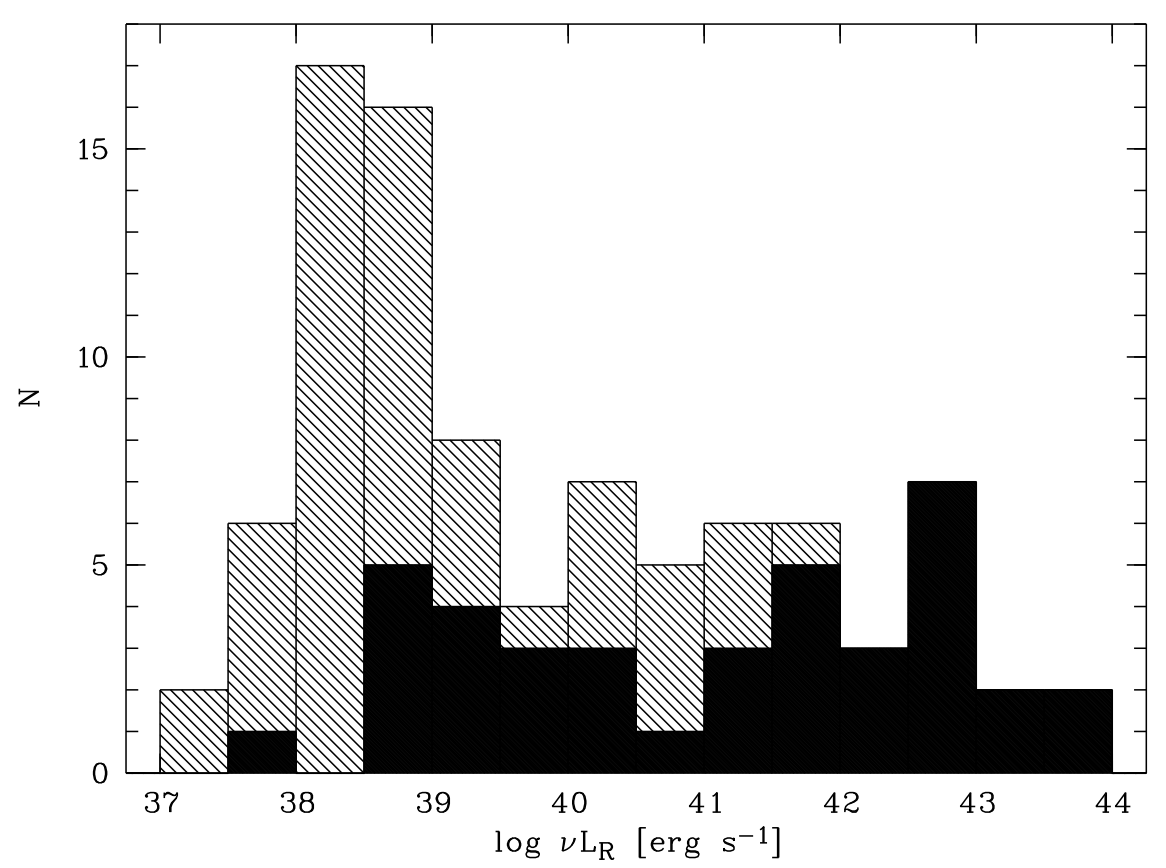

Abbildung 4.8: Verteilung der Radioleuchtkräfte $v \mathrm{~L}_{R}$. Die VBLG sind schwarz, die XGAL schraffiert dargestellt; man sieht deutlich, dass erstere das obere Ende der Leuchtkraftverteilung dominieren. Ihre Verteilung ist im Vergleich zu den XGAL deutlich breiter.

es gibt offensichtlich keinen festen Zusammenhang zwischen den VBLG-Kriterien im Optischen und im Radiobereich.

Neben diesen möglichen intrinsischen Ursachen können immer auch die im den vorangegangenen Abschnitten beschriebenen Grenzen der Radiokataloge hinsichtlich Empfindlichkeit, Himmelsüberdeckung und Kontamination Ursache für ein fehlendes RadioGegenstück sein. Neben den fünf VBLG ist dies auch der wahrscheinliche Grund für das Fehlen von Radiodaten eines BL Lac Objektes, die ansonsten ebenfalls starke Radiostrahler sind.

Wie nicht anders zu erwarten ist unter den nicht aktiven Galaxien der Anteil von Objekten mit Radiodaten am geringsten: er beträgt nur $25 \%$.

\section{Die Radioleuchtkraft}

Durch die Technik der Empfänger bedingt misst man im Radiobereich Flussdichten $\mathrm{f}_{v}$ in Jansky, d.h. es handelt sich dabei um Strahlungsflüsse pro Frequenzeinheit. Berechnet man daraus gemäß Formel (4.3) eine Leuchtkraft, so ist auch das eine Leuchtkraft 
Tabelle 4.6: Statistik der Radioleuchtkräfte: Aufgelistet sind für das Gesamtsample und die Untergruppen VBLG sowie XGAL, XAGN und XAGN-1 die Anzahl der Objekte mit Radioleuchtkräften N sowie Minimum, Maximum, Mittelwert und Standardabweichung von $\log v \mathrm{~L}_{\mathrm{R}}$.

\begin{tabular}{lccccc}
\hline \hline & & \multicolumn{4}{c}{$\log v \mathrm{~L}_{\mathrm{R}}\left[\mathrm{erg} \mathrm{s}^{-1}\right]$} \\
& $\mathrm{N}$ & Min. & Max. & $<\log v \mathrm{~L}_{\mathrm{R}}>$ & $\sigma$ \\
\hline Gesamtsample & 91 & 37.2 & 43.6 & 39.9 & 1.7 \\
VBLG & 39 & 37.8 & 43.6 & 41.1 & 1.7 \\
XGAL & 52 & 37.2 & 41.9 & 39.0 & 1.2 \\
XAGN & 46 & 37.2 & 41.9 & 38.9 & 1.2 \\
XAGN-1 & 29 & 37.4 & 41.9 & 38.8 & 1.2 \\
\hline \hline
\end{tabular}

pro Frequenzeinheit $\mathrm{L}_{v}$ (monochromatische Leuchtkraft). Um mit einer Leuchtkraft im üblichen Sinne - also im physikalischen Sinne einer Leistung - zu arbeiten, betrachtet man gewöhnlich $v \mathrm{~L}_{v}$. Zur Unterscheidung von den Leuchtkräften der anderen Spektralbereiche wird diese Größe hier als $v \mathrm{~L}_{R}$ bezeichnet.

Abbildung 4.8 zeigt die Verteilung der Radioleuchtkräfte des Samples. Man sieht, dass die schwarz dargestellten VBLG das obere Ende der Leuchtkraftverteilung dominieren. Dieses war auch zu erwarten, da hohe Radioleuchtkräfte eines der wichtigen Kriterien für diesen Objekttyp sind.

Neben dem absolut höheren Radioleuchtkräften der VBLG im Vergleich zu den XGAL ist deren breitere Verteilung auffällig: sie erstreckt sich praktisch über den gesamten Bereich von $10^{37}$ bis $10^{44} \mathrm{erg} \mathrm{s}^{-1}$. Die Radioleuchtkräfte der XGAL hingegen zeigen ein deutliches Häufigkeitsmaximum zwischen $10^{38}$ und $10^{39} \mathrm{erg} \mathrm{s}^{-1}$ und konzentrieren sich insgesamt sehr viel stärker um diesen Bereich.

Dieses spiegelt sich in den in Tabelle 4.6 aufgelisteten statistischen Daten der Radioleuchtkräfte wider: Die VBLG weisen im Vergleich zu den XAGN einen höheren Maximalwert und einen höheren Mittelwert sowie insbesondere auch eine deutlich höhere Standardabweichung auf. Erneut gibt es kaum Unterschiede zwischen XGAL, XAGN und XAGN-1.

Um den Einfluss der Nachweisgrenze der Durchmusterungen abschätzen zu können, sind in Abbildung 4.9 die Radioleuchtkräfte gegen die Rotverschiebung $z$ aufgetragen. Dazu wurde das Flusslimit des NVSS-Kataloges $(2.5 \mathrm{mJy})$ in Abhängigkeit von $z$ in Leuchtkräfte umgerechnet und als gestrichelte Linie dargestellt. Der NVSS ist der hierbei relevante Katalog, da er von den großräumigen Katalogen der empfindlichste ist. 


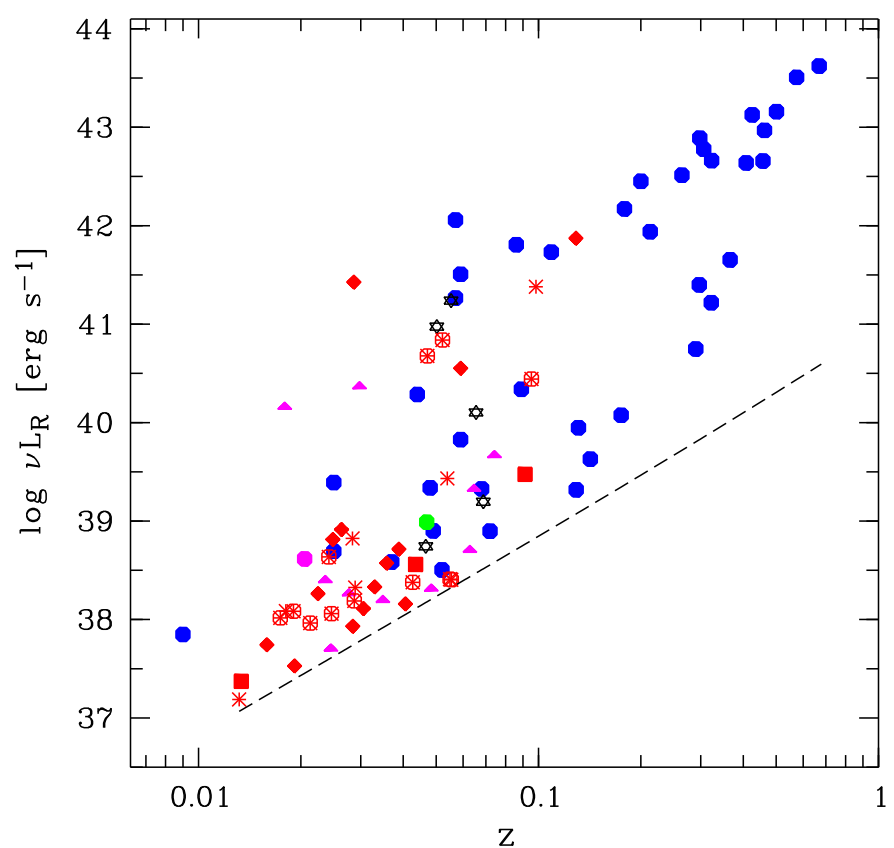

Abbildung 4.9: Die Radioleuchtkraft als Funktion der Rotverschiebung. Die gestrichelte Linie gibt die Nachweisgrenze an. Zu ihrer Berechnung wurde das Flusslimit des NVSS-Kataloges von 2.5 mJy zu Grunde gelegt. Während sich die XGAL überwiegend nahe über der Nachweisgrenze befinden, liegen die VBLG meist deutlich darüber.

Ein erheblicher Teil der XGAL liegt nahe an der Nachweisgrenze. Das legt die Vermutung nahe, dass die XGAL ohne Radio-Gegenstück zu geringe Radiohelligkeiten aufweisen, um in den Durchmusterungen detektiert worden zu sein. Die VBLG hingegen, die im Mittel wesentlich höhere Rotverschiebungen aufweisen, liegen fast alle deutlich über der Nachweisgrenze. Das geht konform mit der Tatsache, dass bis auf wenige Ausnahmen alle VBLG in den Radiodurchmusterungen gefunden wurden.

\subsection{Der Ferninfrarotbereich}

Als Quelle der Ferninfrarotstrahlung in Galaxien werden Staubteilchen angesehen, die ultraviolette und optische Strahlung absorbieren, thermalisieren und dann im Ferninfraroten (FIR) reemittieren.

In einem 3-Komponenten-Modell von Rowan-Robinson (1987) wird die Ferninfrarotemission auf eine Scheibenkomponente (vom Strahlungsfeld normaler Sterne auf etwa $20 \mathrm{~K}$ aufgeheizter Staub), eine Starburst-Komponente (von OB-Sternen auf $50-70 \mathrm{~K}$ 
aufgeheizte Sternentstehungsregion) und eine Seyfert-Komponente (ggf. von einem Aktiven Kern auf etwa $200 \mathrm{~K}$ aufgeheizte Zentralregion) zurückgeführt. Häufig dominiert die Starburst-Komponente.

\subsubsection{Ferninfrarotbeobachtungen}

Beobachtungen im Ferninfrarotbereich sind aufgrund der starken Absorption dieser Strahlung in der Atmosphäre vom Erdboden aus nahezu unmöglich. Im Jahre 1983 hat der Satellit IRAS (Infrared Astronomical Satellite) eine Himmelsdurchmusterung im mittleren und fernen Infrarot durchgeführt und damit diesen Wellenlängenbereich umfassend für die astronomische Forschung zugänglich gemacht (Neugebauer et al. 1984).

IRAS hat in vier breitbandigen Bereichen um die Zentralwellenlängen 12, 25, 60 und $100 \mu \mathrm{m}$ beobachtet und konnte bei seiner Durchmusterung eine nahezu vollständige Himmelsüberdeckung von $96 \%$ erreichen. Insbesondere die Zahl der bekannten extragalaktischen Infrarotquellen konnte mit diesen Beobachtungen um mehrere Größenordnungen gesteigert werden.

Aus dieser Durchmusterung wurde ein Katalog erstellt (IRAS Catalog of Point Sources 1986), der etwa 250000 sicher detektierte Punktquellen enthält (Beichman et al. 1988). Er enthält die Flussdichten in den vier Bändern und ist vollständig bis ca. 0.4 Jy bei $12 \mu \mathrm{m}, 0.5 \mathrm{Jy}$ bei $25 \mu \mathrm{m}, 0.6 \mathrm{Jy}$ bei $60 \mu \mathrm{m}$ und $1.0 \mathrm{Jy}$ bei $100 \mu \mathrm{m}$. Die Positionen haben typischerweise Genauigkeiten von $2^{\prime \prime}$ bis $6^{\prime \prime}$. Es ist nach wie vor der einzige Katalog einer großräumigen Infrarot-Himmelsdurchmusterung.

In diesem Katalog wurden die Ferninfrarot-Daten für alle Sample-Quellen gesucht. 41 dieser Galaxien bzw. 27\% sind im IRAS-Katalog mit einem $60 \mu \mathrm{m}$ und $100 \mu \mathrm{m}$ Fluss enthalten. Abbildung 4.10 zeigt nach Typen differenziert den jeweiligen Anteil an Quellen mit IRAS-Daten als schraffiert dargestellten Bereich.

Bei den Typ-1 Objekten (VBLG, Seyfert-1 und Seyfert-1.5... 1.9) liegt der Anteil von Quellen mit FIR-Daten bei jeweils etwa 30\%. Die entsprechenden Anteile sind hingegen sehr viel kleiner bei den anderen größeren Gruppen der LLAGN (6\%) und der nicht aktiven Galaxien (10\%).

\subsubsection{Der FIR-Datensatz}

Der Ferninfrarotfluss $\mathrm{f}_{\mathrm{FIR}}$ wurde nach Helou et al. (1985) aus den in Jansky gegebenen Flüssen der $60 \mu \mathrm{m}\left(f_{60}\right)$ und $100 \mu \mathrm{m}\left(f_{100}\right)$ IRAS-Bänder berechnet:

$$
f_{F I R}=1.26 \cdot 10^{-11} \mathrm{erg} \mathrm{s}^{-1} \mathrm{~cm}^{-2}\left(2.58 f_{60}+f_{100}\right)
$$




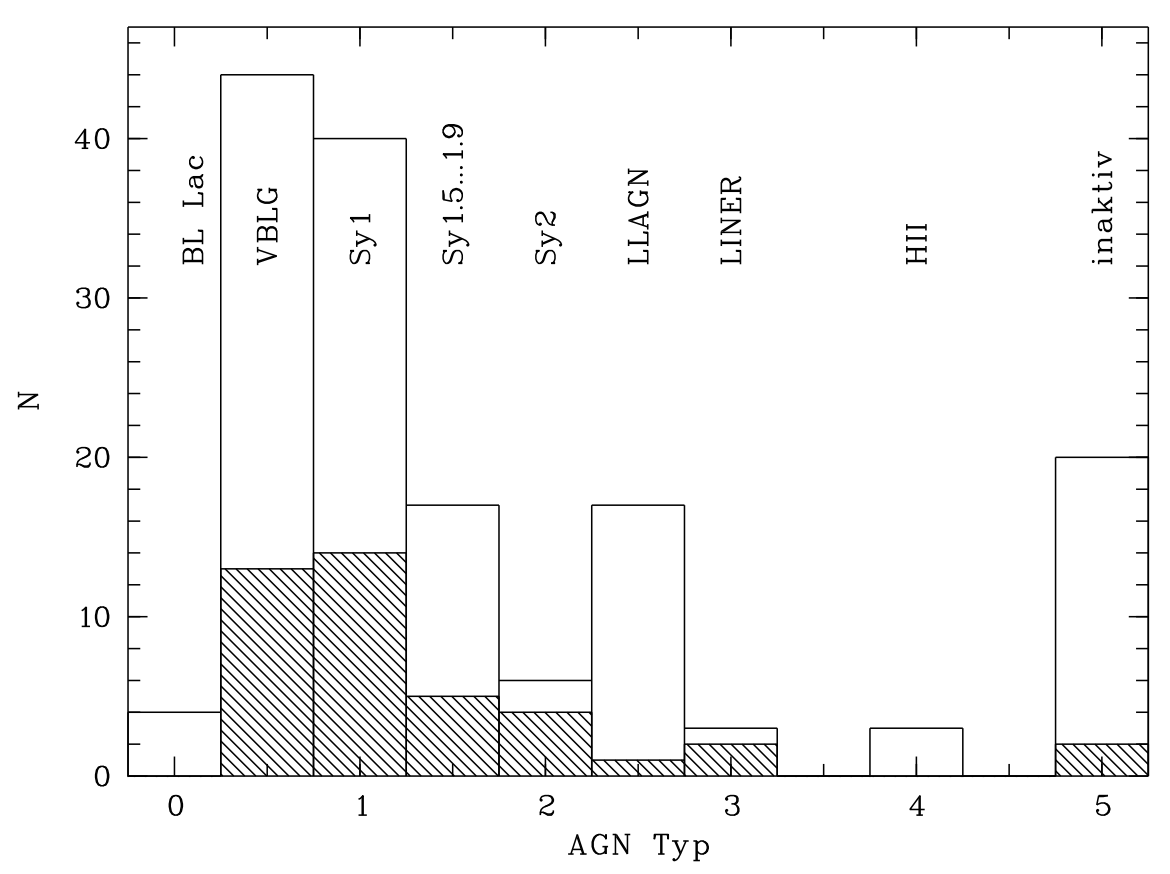

Abbildung 4.10: Es liegen für 41 Objekte bzw. 27\% des Samples FerninfrarotFlüsse des IRAS-Satelliten vor. In diesem Histogramm ist für die verschiedenen AGN-Typen der Anteil von Quellen mit FIR-Daten schraffiert dargestellt. Die prozentualen Anteile sind wie folgt: VBLG 31\%, Sy1 35\%, Sy1.5... 1.9 29\%, Sy2 67\%, LLAGN 6\%, LINER 67\%, inaktiv 10\%.

Aus diesem Fluss wiederum wurde mit Gleichung 4.3 die Ferninfrarotleuchtkraft LIR $_{\text {FIR }}$ bestimmt. Die Häufigkeitsverteilung der so erhaltenen FIR-Leuchtkräfte ist in Abbildung 4.11 dargestellt. Die VBLG sind dabei schwarz, die XGAL schraffiert wiedergegeben.

Auch im Ferninfraroten sind die leuchtkräftigsten Objekte VBLG; sie weisen eine sehr breite Verteilung über fast den gesamten Leuchtkraftbereich von etwa $10^{43}$ bis $10^{46}$ $\mathrm{erg} \mathrm{s}^{-1}$ auf. Die XGAL hingegen konzentrieren sich sehr viel stärker um ihre mittlere FIR-Leuchtkraft bei etwa $10^{44} \mathrm{erg} \mathrm{s}^{-1}$. Das spiegelt sich wieder in den stark unterschiedlichen Standardabweichungen von $\log \mathrm{L}_{F I R}$ der beiden Untergruppen von 0.8 (VBLG) bzw. 0.4 (XGAL). Die statistischen Charakteristika der FIR-Leuchtkräfte sind in Tabelle 4.7 zusammengefasst

Die mittlere FIR-Leuchtkraft für das Gesamtsample beträgt $<\log \mathrm{L}_{\mathrm{FIR}}>=44.1 \pm 0.7$ $\left[\mathrm{erg} \mathrm{s}^{-1}\right.$ ]. Das läßt sich mit der von Worthey (1994) gegebenen bolometrischen Sonnenleuchtkraft von 


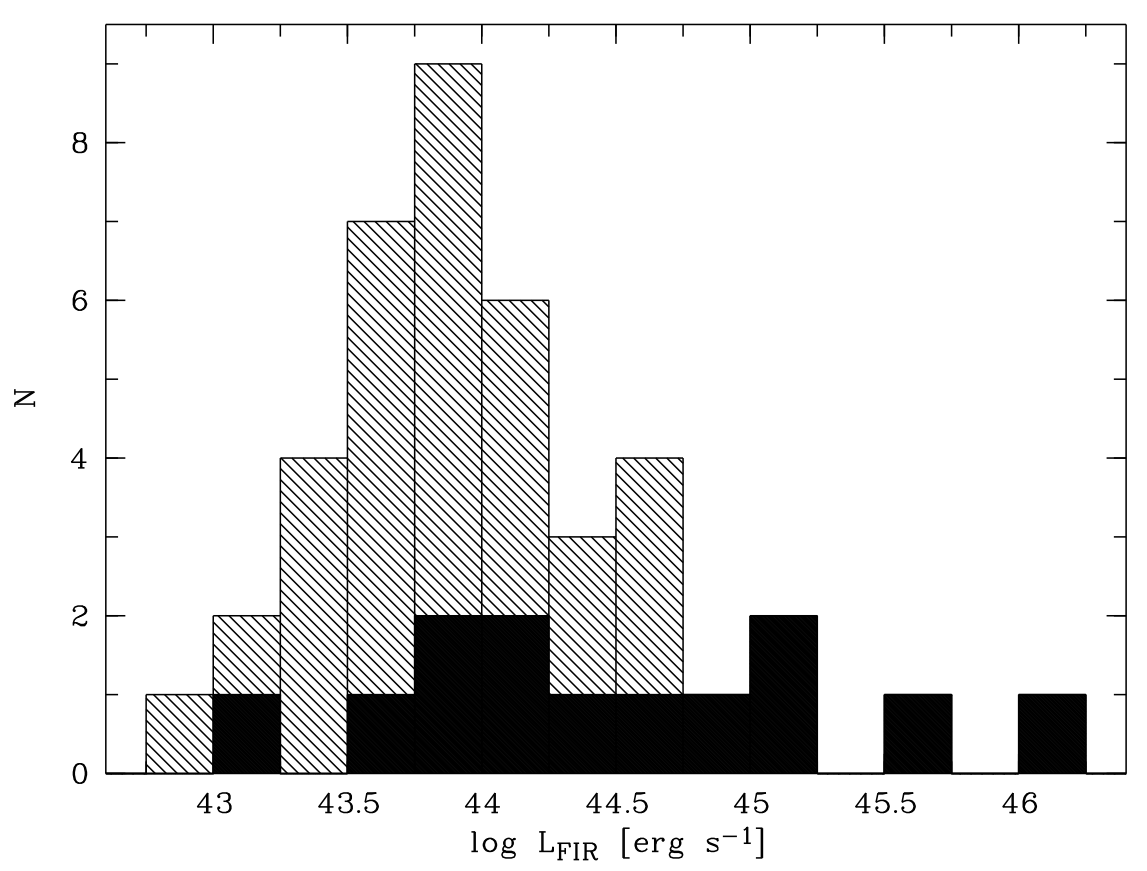

Abbildung 4.11: Die Häufigkeitsverteilung der Ferninfrarot-Leuchtkräfte. Insgesamt liegen für 41 Objekte Ferninfrarot-Leuchtkräfte vor. Schwarz dargestellt sind die VBLG, schraffiert die röntgenselektierten XGAL. Auch im Ferninfraroten sind die leuchtkräftigsten Objekte VBLG.

Tabelle 4.7: Statistik der FIR-Leuchtkräfte: aufgelistet sind für das Gesamtsample und die Untergruppen VBLG sowie XGAL, XAGN und XAGN-1 die Anzahl der Objekte mit FIR-Leuchtkräften N sowie Minimum, Maximum, Mittelwert und Standardabweichung von $\log \mathrm{L}_{\mathrm{FIR}}$.

\begin{tabular}{lccccc}
\hline \hline & & \multicolumn{4}{c}{$\log \mathrm{L}_{\mathrm{FIR}}\left[\mathrm{erg} \mathrm{s}^{-1}\right]$} \\
& $\mathrm{N}$ & Min. & Max. & $<\log \mathrm{L}_{\text {FIR }}>$ & $\sigma$ \\
\hline Gesamtsample & 41 & 42.9 & 46.1 & 44.1 & 0.7 \\
VBLG & 13 & 43.1 & 46.1 & 44.5 & 0.8 \\
XGAL & 28 & 42.9 & 44.6 & 43.8 & 0.4 \\
XAGN & 26 & 42.9 & 44.6 & 43.8 & 0.5 \\
XAGN-1 & 19 & 43.1 & 44.5 & 43.9 & 0.4 \\
\hline \hline
\end{tabular}




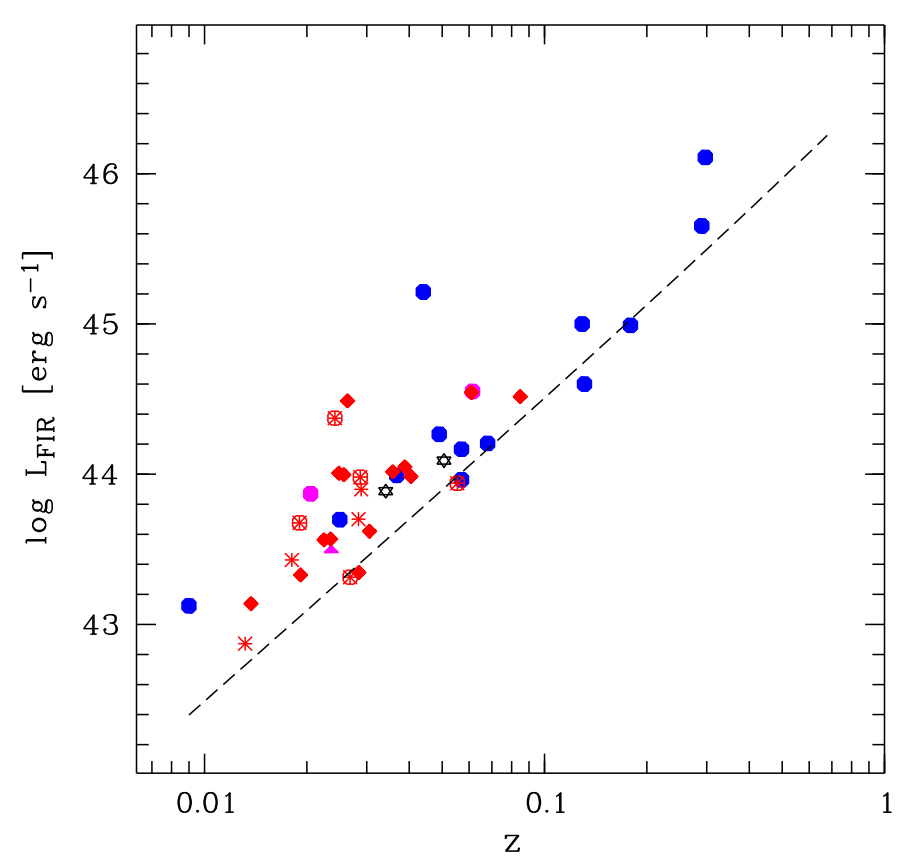

Abbildung 4.12: Ferninfrarot-Leuchtkraft aufgetragen gegen die Rotverschiebung. Die gestrichelte Linie gibt eine Abschätzung für die Grenzempfindlichkeit des IRAS-Kataloges an (siehe Text). Die meisten Quellen befinden sich nahe am Detektionslimit.

$$
L_{\odot}=3.826 \cdot 10^{33} \mathrm{erg} \mathrm{s}^{-1}
$$

$\mathrm{zu}<\log \mathrm{L}_{\mathrm{FIR}}>=10.5 \pm 0.7\left[\mathrm{~L}_{\odot}\right]$ umrechnen.

Im Vergleich dazu finden Lehnert \& Heckman (1995) für ihr Sample von etwa 50 infrarothellen Starburst-Galaxien einen entsprechenden Mittelwert von $<\log \mathrm{L}_{I R}>=$ $10.64 \pm 0.61\left[\mathrm{~L}_{\odot}\right]$, wobei $\mathrm{L}_{I R}$ nur wenig von $\mathrm{L}_{\mathrm{FIR}}$ abweicht. Klaas (1988) gibt für sein Sample optisch schwacher Infratrotgalaxien keine mittlere FIR-Leuchtkraft an, aber auch seine Quellen liegen im Bereich $\log \mathrm{L}_{\mathrm{FIR}}=10 \ldots 12$ [L $\odot$ ]. Unser Sample ist somit bezüglich der mittleren FIR-Eigenschaften den beiden genannten Samples sehr ähnlich, was sicherlich auch dadurch bedingt ist, dass auch diese Samples auf IRASBeobachtungen beruhen.

Abbildung 4.12 zeigt die FIR-Leuchtkraft aufgetragen gegen die Rotverschiebung. Dazu ist eine Abschätzung der Grenzempfindlichkeit des IRAS-Kataloges als gestrichelte Linie eingezeichnet. Zu ihrer Berechnung wurde als Empfindlichkeit in den einzelnen Bändern jeweils 50\% der oben angegebenen Flussgrenzen angenommen, bis zu denen der Katalog vollständig ist, das heißt 0.6 Jy bei $60 \mu \mathrm{m}$ bzw. 1.0 Jy bei $100 \mu \mathrm{m}$. 
Es konnten allerdings einige wenige Objekte leicht unterhalb dieser so abgeschätzten Grenzempfindlichkeit detektiert werden, wenn sie sich z.B. in Regionen mit besonders geringer Hintergrundkontamination befinden. Die meisten Quellen befinden sich nahe am Detektionslimit.

Zusammenfassend kann festgestellt werden, dass einerseits der FIR-Datensatz unter den Datensätzen der hier diskutierten vier Spektralbereiche der kleinste ist, was bei den Betrachtungen und Vergleichen in den folgenden Kapiteln berücksichtigt werden muss. Anderseits ist das vorliegende Sample bezüglich der mittleren FIR-Leuchtkraft den Samples von Lehnert \& Heckman (1995) (Starburst-Galaxien) sowie Klaas (1988) (optisch schwache Infrarotgalaxien) sehr ähnlich. Daraus kann man folgern, dass nicht etwa eine unterdurchschnittliche FIR-Emission der Quellen unseres Samples, sondern die relativ zu den anderen Durchmusterungen geringere Empfindlichkeit des IRAS-Kataloges ausschlaggebend ist.

\subsubsection{Die Ferninfrarotfarbe}

Da IRAS in vier Bändern beobachtet hat, enthalten seine Daten auch spektrale Informa-

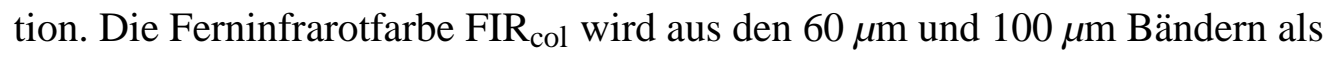

$$
F I R_{c o l}=\log \frac{f_{60}}{f_{100}}
$$

berechnet.

Unter der Annahme thermischer Emission läßt sich aus der FIR-Farbe auf die Temperatur des emittierenden Staubs schließen. Helou et al. (1985) benutzen das lineare Verhältnis $R=f_{60} / f_{100}$ und geben damit folgende Abgrenzung von kaltem und warmem Staub an:

$$
\begin{array}{llll}
\text { kalt }: & \mathrm{R}<0.4 & \text { bzw. } & \text { FIR }_{\mathrm{col}}<-0.4 \\
\text { warm : } & \mathrm{R}>0.5 & \text { bzw. } & \text { FIR }_{\mathrm{col}}>-0.3
\end{array}
$$

FIR-Farben, die dazwischen liegen $\left(0.4<R<0.5\right.$ bzw. $\left.-0.4<\mathrm{FIR}_{\mathrm{col}}<-0.3\right)$, bezeichnen sie dementsprechend als intermediär. Klaas (1988) rechnet FIR col $_{\text {über eine }}$ modifizierte Planck-Funktion direkt in eine Farbtemperatur bzw. die Staubtemperatur um.

Abbildung 4.13 zeigt die Ferninfrarotleuchtkraft als Funktion der FIR-Farbe. Obige Abgrenzungen von kalten und warmen Staubtemperaturen sind als senkrechte Geraden eingezeichnet - links im Diagramm befinden sich die kalten, rechts die warmen Quellen.

Da in erster Näherung die Ferninfrarotleuchtkraft nur von der Gesamtstaubmasse $\mathbf{M}_{D}$ und der Staubtemperatur $\mathrm{T}_{D}$ wie 


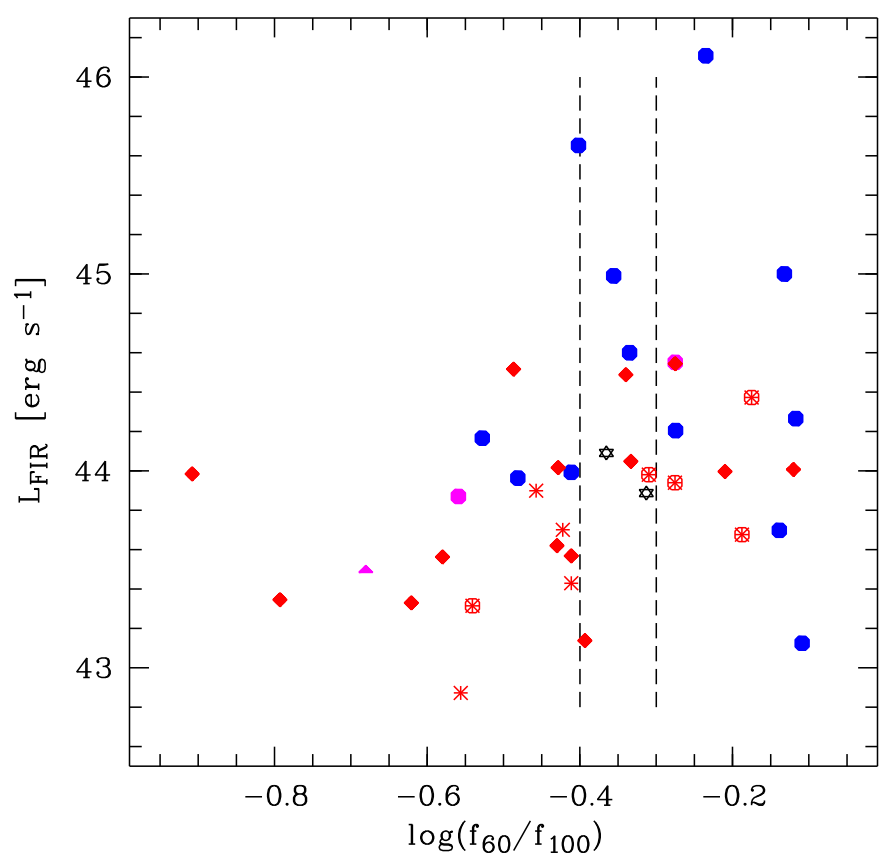

Abbildung 4.13: Ferninfrarotleuchtkraft gegen FIR-Farbe. Die senkrechten Linien trennen kalte (links), intermediäre und warme Quellen (rechts) nach der Definition von Helou et al. (1985). Da die FIR-Farbe ein Maß für die Staubtemperatur ist, sollte $\mathrm{L}_{\mathrm{FIR}}$ bei konstanter Gesamtstaubmasse mit FIR $_{\text {col }}$ ansteigen. Die Korrelationsanalyse bestätigt eine positive Korrelation von $\mathrm{L}_{\mathrm{FIR}}$ und FIR $\mathrm{col}_{\text {. }}$.

$$
L_{F I R} \propto M_{D} \cdot T_{D}^{5}
$$

abhängt (Soifer, Neugebauer \& Houck 1987, Draine \& Lee 1984), wäre ein starker Anstieg von LFIR mit der FIR-Farbe zu erwarten. Das ist in Abbildung 4.13 nur andeutungsweise zu erkennen. Die Korrelationsanalyse ergibt allerdings tatsächlich eine positive Korrelation der beiden Größen ( $\mathrm{r}=0.32, \mathrm{P}=0.04)$.

Eine lineare Korrelation zwischen $\mathrm{L}_{\mathrm{FIR}}$ und der FIR-Farbe finden auch Iyengar, Rengarajan \& Verma (1986) für ein Sample aus 86 Spiralgalaxien, und auch Lehnert \& Heckman (1995) zeigen eine entsprechende Abbildung, während van den Broek et al. (1991) in einem $\mathrm{L}_{\mathrm{FIR}}$-FIR $\mathrm{col}_{\mathrm{co}}$-Diagramm insbesondere die Auswirkungen des IRAS-Flusslimits aufzeigen, eine deutliche Korrelation hingegen nicht erkennen lassen.

Es sei noch darauf hingewiesen, dass sowohl die Ableitung einer Staubtemperatur aus der FIR-Farbe wie auch die Abschätzung einer totalen IR-Leuchtkraft mit Hilfe eines

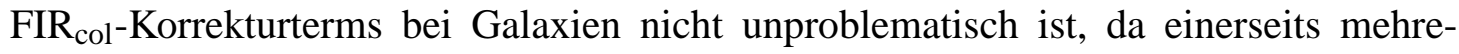
re Komponenten mit unterschiedlichen Temperaturen an der Emission beteiligt sein 
können (Helou et al. 1988), andererseits häufig sowohl sehr kalte Staubkörner (Cox, Krügel \& Mezger 1986) als auch sehr kleine Staubkörner mit variabler Temperatur (Boulanger et al. 1988) gleichzeitig vorliegen und zur Emission beitragen. Das macht die quantitative Analyse schwieriger, ändert aber nichts an der Tatsache, dass die FIRFarbe zumindest ein qualitatives Maß für die Staubtemperatur in Galaxien ist.

\subsection{Der Malmquist-Bias}

Da sich die Quellen dieses Samples über einen großen Entfernungs- bzw. Rotverschiebungsbereich erstrecken, ist die Abhängigkeit der Beobachtungsgrößen von der Rotverschiebung von fundamentaler Bedeutung für das Verständnis ihrer intrinsischen Eigenschaften. Diese Abhängigkeit wird im Wesentlichen durch das Detektionslimit der Beobachtungsinstrumente einerseits und die Leuchtkraftfunktionen der Quellen andererseits bestimmt. Die Leuchtkraftfunktion gibt die Raumdichte als Funktion der absoluten Leuchtkraft an.

Zur besseren Vergleichbarkeit sind die hier betrachteten Leuchtkräfte in Abbildung 4.14 noch einmal gemeinsam als Funktion der Rotverschiebung aufgetragen. Es fällt sofort auf, dass im Mittel alle vier Leuchtkräfte jeweils mit der Rotverschiebung ansteigen. Diesen in flusslimitierten Beobachtungen unvermeidlichen Effekt bezeichnet man nach Arbeiten von Malmquist (1920, 1924) als Malmquist-Bias. Er wurde z.B. von Gonzalez \& Faber (1997) und Teerikorpi (1997) ausführlich diskutiert.

Es fehlen in Abbildung 4.14 sowohl Quellen mit hoher Rotverschiebung und geringer Leuchtkraft wie auch Quellen mit niedriger Rotverschiebung und hoher Leuchtkraft.

Ersteres liegt daran, dass diese Quellen nicht detektierbar sind, weil sie unter der Nachweisgrenze liegen: bei einer vorgegebenem Nachweisgrenze des messbaren Flusses steigt die gerade noch detektierbare Leuchtkraft mit der Rotverschiebung wie in Abschnitt 4.2 beschrieben mit dem Quadrat der Leuchtkraftentfernung an. Dies wird auch eindeutig durch die in den Graphiken eingezeichneten Abschätzungen für die Nachweisgrenze illustriert.

Letzteres liegt wiederum daran, dass die Quellen umso seltener sind, je leuchtkräftiger sie sind (Leuchtkraftfunktion). Mit zunehmender Rotverschiebung steigt das betrachtete Raumvolumen und enthält dann auch leuchtkräftigere Quellen. Besonders leuchtkräftige Objekte werden daher nicht bei sehr niedriger Rotverschiebung (und somit sehr kleinem betrachtetem Raumvolumen) beobachtet.

Die soeben beschriebenen Effekte sind sowohl für das hier vorliegende Sample in allen vier betrachteten Frequenzbändern wie auch für das zum Vergleich eingezeichnete Veron-Sample in den absoluten optischen Magnituden leicht zu erkennen. 

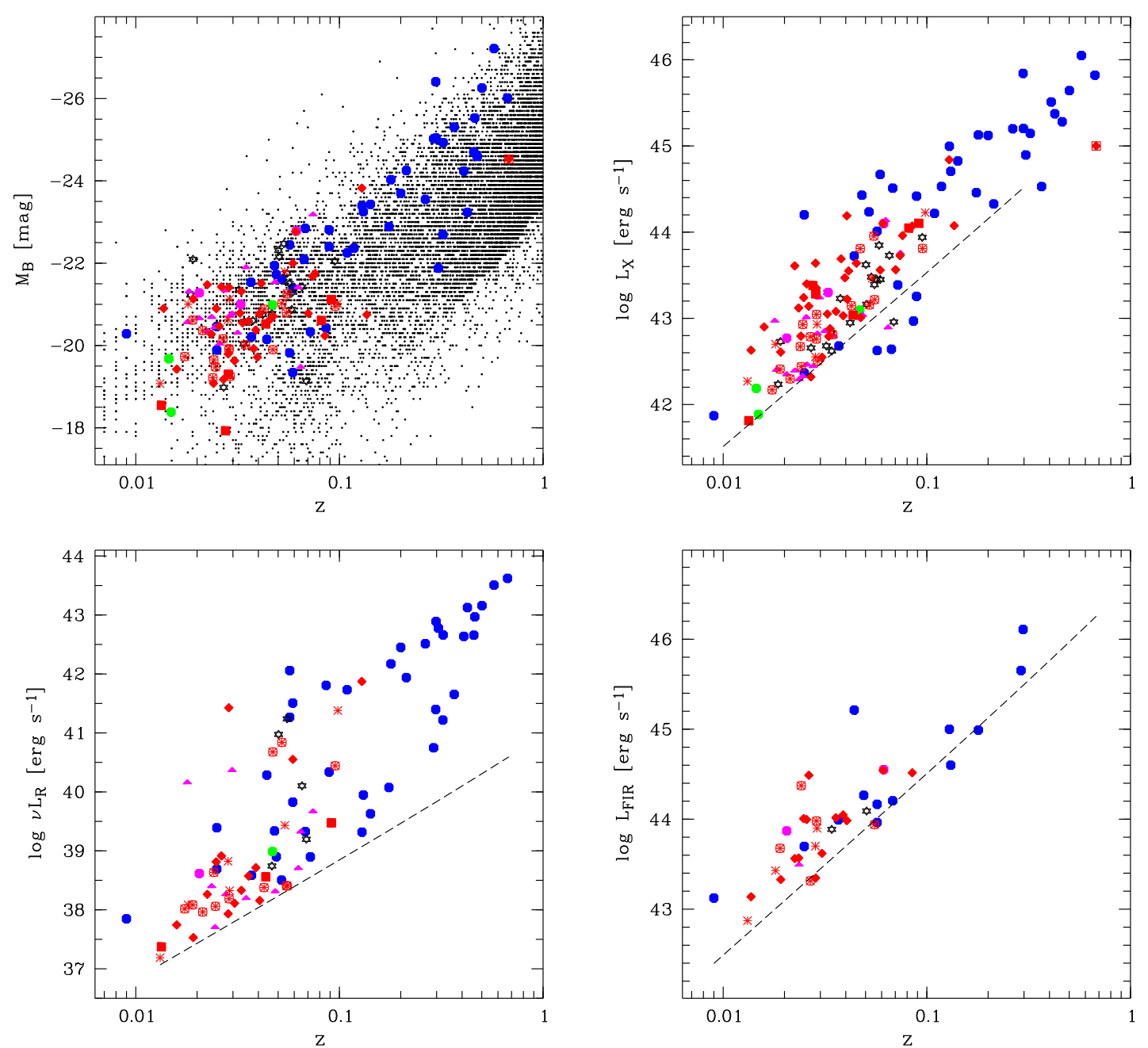

Abbildung 4.14: Die Leuchtkräfte der vier Frequenzbereiche als Funktion der Rotverschiebung. Wie in den vorherigen Abschnitten beschrieben sind zusätzlich Abschätzungen für das Detektionslimit bzw. im Fall der optischen Magnituden $\mathrm{M}_{\mathrm{B}}$ zum Vergleich die Quellen des Veron-Kataloges eingezeichnet. Der Anstieg der Leuchtkräfte mit der Rotverschiebung ist in allen Fällen offensichtlich und wird als Malmquist-Bias bezeichnet.

Zum Vergleich sind in Abbildung 4.15 die entsprechenden vier Flüsse als Funktion der Rotverschiebung gezeigt. Da der Fluss eine entfernungsabhängige Messgröße ist und die oben für Leuchtkräfte aufgeführten Argumente nicht gelten, ist in erster Näherung kein Zusammenhang von Fluss und Rotverschiebung erkennbar. Insbesondere bei der scheinbaren optischen Magnitude $m_{B}$ sieht man bei genauerer Betrachtung sowohl für das eigene Sample, aber noch besser für die Veron-Galaxien, dass mit zunehmender 

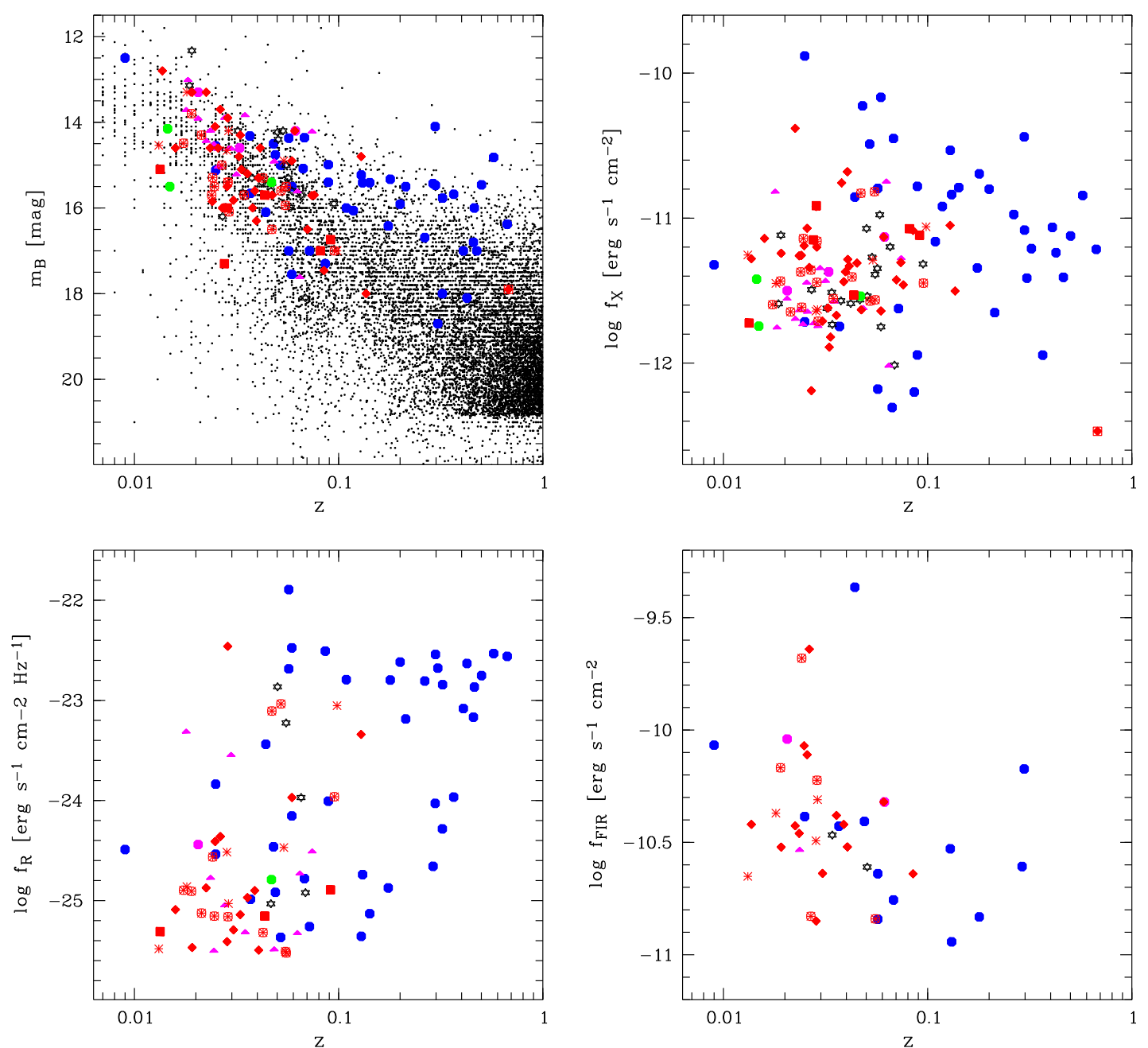

Abbildung 4.15: Die Flüsse der vier Frequenzbereiche als Funktion der Rotverschiebung. Diese Abbildungen entsprechen dem so genannten Hubblediagramm. Für gleichartige Objekte mit konstanter absoluter Helligkeit (,Standardkerzen“) würden sich gemäß der Hubble-Beziehung (Gleichung 4.13) lineare Zusammenhänge ergeben. Das ist hier nur für $m_{B}$ relativ gut und für $f_{F I R}$ andeutungsweise zu erkennen.

Rotverschiebung die maximale scheinbare Helligkeit abnimmt - eine offensichtliche, rein geometrische Folge davon, dass die intrinsische, absolute Leuchtkraft limitiert ist. Dass bei geringer Rotverschiebung mehr scheinbar helle Quellen beobachtet werden, liegt daran, dass diese leichter detektierbar sind. Hinzu kommt, dass natürlich auch die minimale absolute Leuchtkraft der für dieses Sample in Frage kommenden Objekte limitiert ist. 
Eine Auftragung der scheinbaren optischen Helligkeit $m$ gegen $\log z$ nennt man auch Hubblediagramm. In diesem Diagramm spiegelt sich die aus den Gleichungen 4.5 und 4.7 abgeleitete Hubble-Beziehung

$$
m=5 \log c z+5 \log \psi+M-5 \log H_{0}+25
$$

wider. Für gleichartige Objekte mit konstanter absoluter Helligkeit M, so genannte „Standardkerzen“, ergibt sich im nahen Kosmos $(\psi \approx 1)$ eine Gerade (Unsöld \& Baschek 1988, Abbildung 5.9.3). Auf kosmologischen Skalen kann man prinzipiell aus der Abweichung von einer Geraden bei hohen Rotverschiebungen auf $\psi$ schließen.

Die Objekte unseres Samples sind keine Standardkerzen, sondern ihre intrinsischen Leuchtkräfte streuen relativ stark (s. Abbildung 4.14). Ferner ist der überdeckte Wertebereich der gemessenen Flüsse jeweils deutlich kleiner als der Wertebereich der entsprechenden Leuchtkraft. Daher ist die lineare Beziehung gemäß Gleichung 4.13 nur für $m_{B}$ andeutungsweise zu erkennen, während für alle Leuchtkräfte der Gang mit der Rotverschiebung offensichtlich ist.

Die scheinbare optische Helligkeit war für alle Quellen des Samples ein (indirektes) Selektionskriterium, da nur gut spektroskopierbare Galaxien in das Sample aufgenommen werden konnten. Darum ist in diesem Fall die optische Helligkeit aussagekräftiger als die Flüsse der anderen Frequenzbereiche.

Abbildung 4.14 zeigt auch, dass die Streuung der Leuchtkräfte groß gegenüber potenziellen K-Korrekturen ist. Da für diese außerdem zusätzliche Annahmen über den Spektralverlauf nötig gewesen wären, wurde darauf verzichtet.

Vorausblickend sei an dieser Stelle bereits erwähnt, dass die Betrachtung von Flussbzw. Leuchtkraftverhältnissen eine wirkungsvolle Methode ist, alle hier diskutierten, rein entfernungsabhängigen Effekte zu eliminieren (s. Kapitel 5). 


\section{Kapitel 5}

\section{Flussverhältnisse und Korrelationen}

Im folgenden Abschnitt werden die integrierten Flüsse der vier Spektralbereiche Radio (R), Ferninfrarot (FIR), Optisch (B) und Röntgen (X) im Zusammenhang betrachtet und analysiert. Es werden insbesondere Abhängigkeiten und Korrelationen zwischen den vier Bändern untersucht.

Große Bedeutung haben die Flussverhältnisse $f_{A / B}$, die aus den integrierten Flüssen jeweils zweier Bänder A und B gebildet werden:

$$
f_{A / B}=\frac{f_{A}}{f_{B}} .
$$

Diese haben den Vorteil, dass man einen neuen Parameter erhält, der die Eigenschaften in zwei Frequenzbändern gleichzeitig widerspiegelt und der leicht mit dritten Parametern verglichen werden kann.

Ein weiterer Vorteil besteht darin, dass ein Flussverhältnis prinzipiell entfernungsunabhängig ist. Es ist äquivalent zum entsprechenden Leuchtkraftverhältnis, kann jedoch auch berechnet werden, wenn die Entfernung und damit die Leuchtkraft eines Objektes unbekannt sind. Für das Flussverhältnis ist es weiterhin unwesentlich, ob das betrachtete Objekt absolut leuchtkräftig oder leuchtschwach ist.

Die Flussverhältnisse in dieser Arbeit wurden soweit möglich so gebildet, dass vergleichbare Werte in der Literatur zur Verfügung standen. In der Literatur wird ferner auch der sog. Spektralindex $\alpha$ verwendet. Er wird über ein spektrales Potenzgesetz der Art

$$
f_{v} \propto v^{-\alpha}
$$

definiert. Damit ist der Spektralindex zwischen den Frequenzbändern A und B proportional zum hier verwendeten Logarithmus des Flussverhältnisses $f_{A / B}$ : 


$$
\alpha_{A B} \propto \log f_{A / B}
$$

$\mathrm{Ab}$ hier werden nur noch Aktive Galaxien betrachtet, d.h. neben den VBLG die bisher bereits als XAGN bezeichnete Untergruppe der röntgenselektierten Aktiven Galaxien. Die röntgenselektierten inaktiven und H II-Galaxien werden hier nicht weiter berücksichtigt. Die Natur der LINER ist nach wie vor nicht sicher geklärt und wird in der Literatur noch kontrovers diskutiert. Es gilt aber als sehr wahrscheinlich, dass auch sie einen Aktiven Kern als Energiequelle beherbergen. Daher werden sie hier zu den Aktiven Galaxien gezählt. Das verbleibende Gesamtsample wird in den folgenden Tabellen kurz mit AGN bezeichnet.

Die in den folgenden Abschnitten angeführten Korrelationsergebnisse werden am Ende dieses Kapitels (Abschnitt 5.8) nochmals geschlossen aufgelistet und im Zusammenhang mit den anderen Resultaten diskutiert.

\subsection{Verhältnis von Röntgen- zu optischem Fluss $\mathbf{f}_{\mathrm{X} / \mathrm{B}}$}

Im Folgenden sollen die Eigenschaften des Samples im Röntgen- und im optischen Spektralbereich ins Verhältnis gesetzt werden. Dazu ist in Abbildung 5.1 der Röntgenfluss gegen die scheinbare optische Helligkeit bzw. die Röntgenleuchtkraft gegen die absolute optische Helligkeit aufgetragen.

Sowohl die graphische Darstellung wie auch die Korrelationsanalyse zeigen eindeutig, dass im ersten Fall (Fluss gegen Fluss) keinerlei Korrelation $(r=-0.02, P=0.79$ ), im zweiten (Leuchtkraft gegen Leuchtkraft) dagegen eine sehr starke Korrelation ( $\mathrm{r}=$ $-0.53, \mathrm{P}<0.001)$ vorliegt.

Letzteres liegt daran, dass bei einer entfernteren Galaxie, deren Flüsse im beobachtbaren Bereich liegen, die Leuchtkräfte entsprechend höher sind als bei einer nahen Galaxie. Diese Korrelation spiegelt daher den Entfernungsbereich wider, den dieses Sample überdeckt, und entspricht dem im Abschnitt 4.7 beschriebenen Zusammenhang zwischen Leuchtkraft und Rotverschiebung (Malmquist-Bias, s. auch Abbildung 4.14) - sie besitzt keine direkte physikalische Bedeutung, sondern ist durch die Beobachtungsmöglichkeiten und die Leuchtkraftfunktion der Galaxien bedingt. Dabei haben die VBLG im Mittel deutlich höhere Entfernungen (und höhere Leuchtkräfte) als die XAGN.

Dieses Verhalten wird sich aus den genannten Gründen bei allen in diesem Abschnitt betrachteten Leuchtkraft-Paaren finden. Interessant ist daher nicht die Korrelation der Leuchtkräfte als solche, sondern die Stärke bzw. Dispersion der Korrelation, die zum 

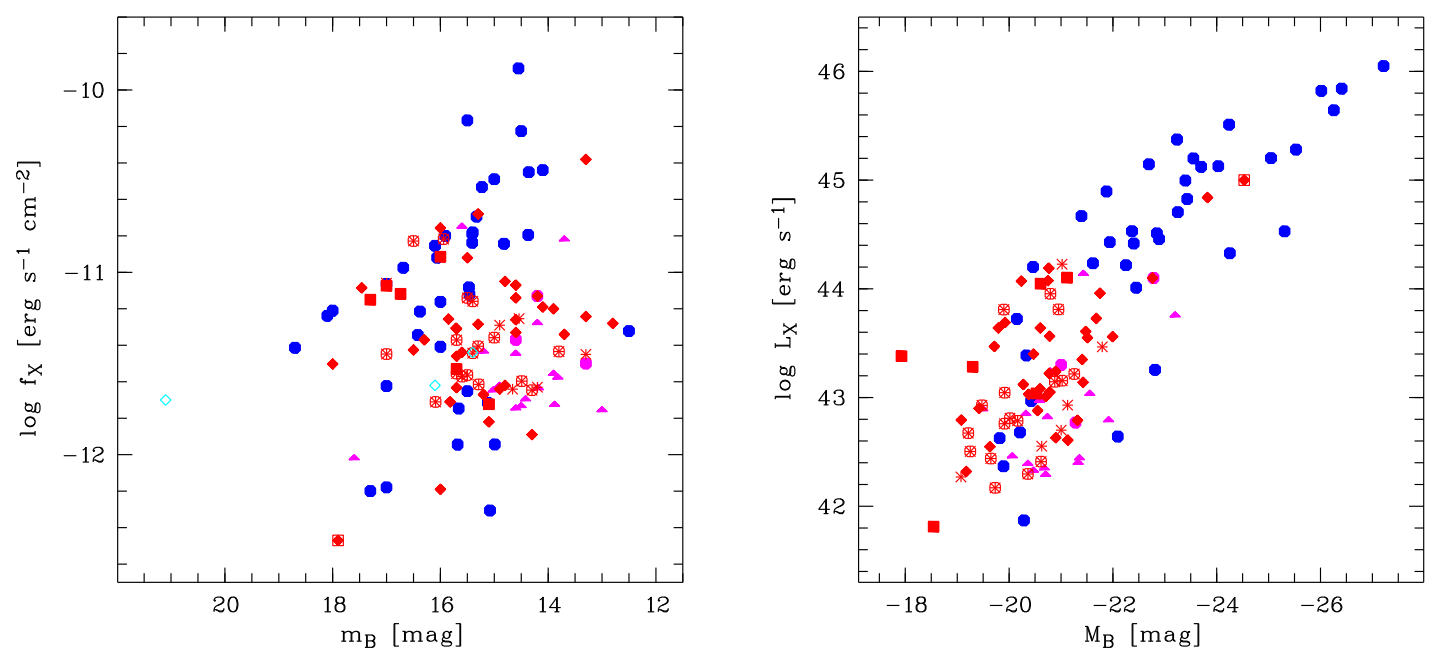

Abbildung 5.1: Röntgenfluss gegen die scheinbare optische Helligkeit (links) bzw. die Röntgenleuchtkraft gegen die absolute optische Helligkeit (rechts). Während die relativen Größen (Flüsse) keinerlei Korrelation zeigen, sind die absoluten Größen (Leuchtkräfte) stark korreliert. Bei niedrigen Leuchtkräften überwiegen die XAGN, bei hohen die VBLG. Wie bereits erwähnt ist die Zeichenkodierung in dieser und in allen entsprechenden Abbildungen wie in der Legende der Abbildung 3.2 erklärt, wobei gefüllte blaue Kreise als Symbol für VBLG sowie offene hellblaue Rauten als Symbol für BL Lac Objekte hinzukommen.

Vergleich mit der entsprechenden Korrelation der jeweiligen Flüsse dienen kann. Bedeutsam sind dagegen eventuelle Korrelationen zwischen den Flüssen in verschiedenen Frequenzbändern und die sich daraus ergebenden Flussverhältnisse.

Das Verhältnis von Röntgen- und optischem Fluss $f_{X / B}$ soll dazu dienen, die Quelle der Strahlung zu charakterisieren. Es wurde entsprechend dem Ansatz von Maccacaro et al. (1988) gemäß

$$
\log f_{X / B}=\log f_{X}+0.4 * m_{B}+5.37
$$

berechnet.

Maccacaro et al. (1988) geben Wertebereiche für dieses Verhältnis für verschiedene Sterntypen, normale Galaxien, AGN sowie BL Lac Objekte an, das in dieser Sequenz zunehmend ist. Die Absolutwerte von Maccacaro et al. (1988) sind nicht exakt mit den hier berechneten vergleichbar, da sie im Röntgenbereich EINSTEIN-Flüsse (0.3 $3.5 \mathrm{keV}$ ) und im Optischen das V-Band verwendet haben. Die Unterschiede zu den hier benutzten Flüssen sollten aber relativ gering sein und beim Vergleich der Größenordnungen zu keinen relevanten Diskrepanzen führen. Maccacaro et al. (1988) finden 

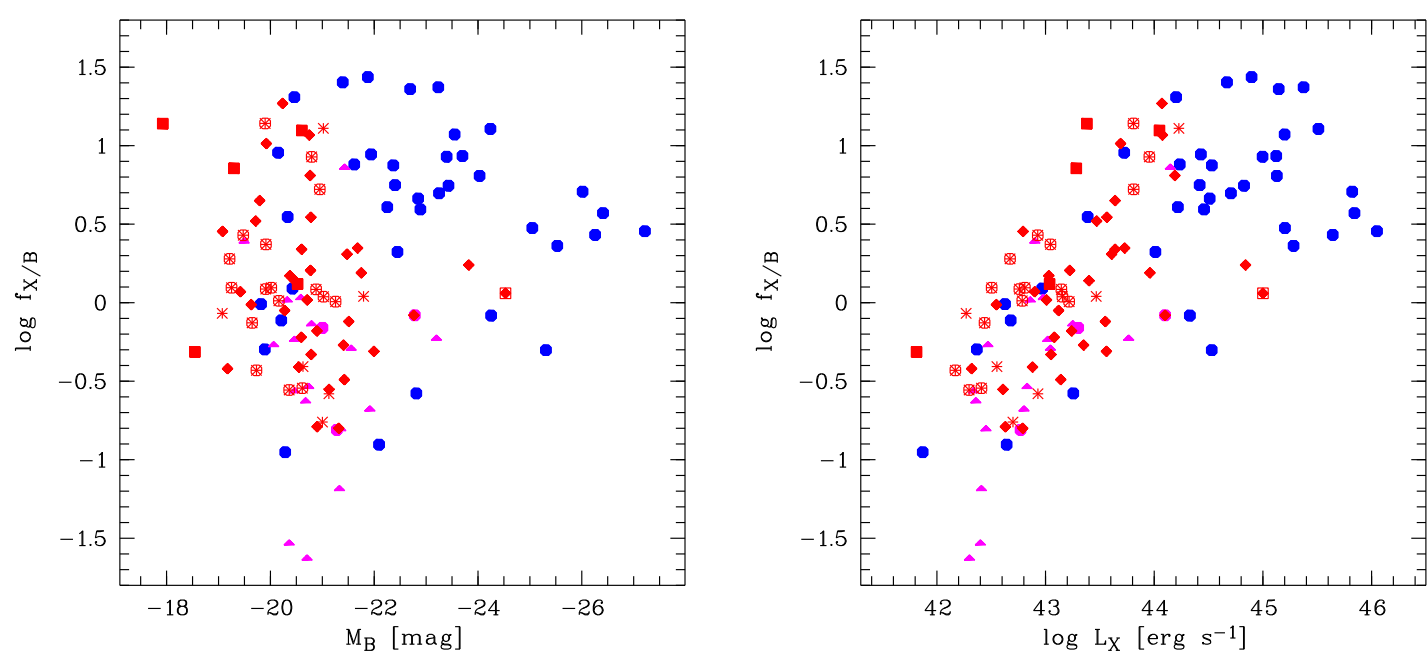

Abbildung 5.2: Das Flussverhältnis $\mathrm{f}_{\mathrm{X} / \mathrm{B}}$ als Funktion der optischen Absoluthelligkeit $\mathrm{M}_{B}$ (links) bzw. der Röntgenleuchtkraft $\mathrm{L}_{\mathrm{X}}$ (rechts). Die VBLG haben im Mittel nicht nur größere optische Absoluthelligkeiten und Röntgenleuchtkräfte sondern auch höhere mittlere $\mathrm{f}_{\mathrm{X} / \mathrm{B}}$ ( $\mathrm{s}$. auch Tabelle 5.1). Dabei kann es sich keinesfalls um einen Selektionseffekt handeln, da ein solcher sich gegenteilig ausgewirkt hätte.

$\log f_{X / V}$ für normale Galaxien im Bereich $-1.8 \ldots-0.6$, für AGN im Bereich $-1.3 \ldots 1.2$ und für BL Lac Objekte im Bereich $0.2 \ldots 1.6$.

Abbildung 5.2 zeigt für das hier vorliegende Sample das Flussverhältnis $\mathrm{f}_{\mathrm{X} / \mathrm{B}}$ als Funktion der optischen Absoluthelligkeit $\mathrm{M}_{B}$ bzw. der Röntgenleuchtkraft $\mathrm{L}_{X}$. Im Mittel sind die VBLG nicht nur im optischen und im Röntgenbereich leuchtkräftiger als die XAGN, sie zeigen außerdem auch ein etwas höheres Flussverhältnis $\log f_{X / B}(0.55 \pm 0.62$ gegenüber $0.03 \pm 0.63$ ). Diese Diskrepanz ändert sich nur unwesentlich, wenn man die VBLG nur mit den Typ-1-Objekten unter den XAGN vergleicht, da deren $\log \mathrm{f}_{\mathrm{X} / \mathrm{B}}$ mit $0.16 \pm 0.51$ nur geringfügig höher liegt.

Weiterhin ist die Streuung der Werte bei den VBLG etwas höher als bei den XAGN. Der Abbildung 5.2 kann man ferner entnehmen, dass nahezu alle hier gefundenen Werte im Rahmen der Genauigkeit mit dem entsprechenden Wertebereichen von Maccacaro et al. (1988) verträglich sind.

Dass das mittlere $\mathrm{f}_{\mathrm{X} / \mathrm{B}}$ der VBLG über dem der XAGN liegt, ist auch deswegen bemerkenswert, da der Röntgenfluss bei den XAGN ein Selektionskriterium war, bei den VBLG hingegen nicht. Ein Selektionseffekt kann daher als Erklärung ausgeschlossen werden, da er sich genau gegenteilig hätte auswirken müssen.

Interessant ist auch der sehr geringe Unterschied des mittleren $\mathrm{f}_{\mathrm{X} / \mathrm{B}}$ der XAGN und der Untergruppe der Typ-1 XAGN (XAGN-1). Um einen möglichen Zusammenhang von 
Tabelle 5.1: Statistik des Flussverhältnis $f_{X / B}$ für das Gesamtsample (AGN) und verschiedene Untergruppen. Eine graphische Darstellung dieser Daten findet sich in Abbildung 5.3.

\begin{tabular}{lrrccc}
\hline \hline & & \multicolumn{4}{c}{$\log \mathrm{f}_{\mathrm{X} / \mathrm{B}}$} \\
& $\mathrm{N}$ & Min. & Max. $<\log \mathrm{f}_{\mathrm{X} / \mathrm{B}}>$ & $\sigma$ \\
\hline AGN & 122 & -1.62 & 2.11 & 0.18 & 0.67 \\
AGN-1 & 93 & -0.95 & 1.44 & 0.31 & 0.59 \\
VBLG & 37 & -0.95 & 1.44 & 0.55 & 0.62 \\
XAGN & 85 & -1.62 & 2.11 & 0.03 & 0.63 \\
XAGN-1 & 56 & -0.80 & 1.27 & 0.16 & 0.51 \\
BL Lac & 3 & 0.09 & 2.11 & 0.80 & 1.14 \\
Sy1 & 39 & -0.80 & 1.27 & 0.16 & 0.54 \\
Sy1.5...1.9 & 17 & -0.56 & 1.14 & 0.16 & 0.47 \\
Sy2 & 6 & -0.76 & 1.11 & -0.11 & 0.67 \\
LLAGN & 17 & -1.62 & 0.87 & -0.43 & 0.64 \\
LINER & 3 & -0.81 & -0.08 & -0.35 & 0.40 \\
\hline \hline
\end{tabular}

$\mathrm{f}_{\mathrm{X} / \mathrm{B}}$ mit dem Aktivitätstyp zu untersuchen, wurden Mittelwerte und Standardabweichungen von $\log f_{X / B}$ für alle Galaxien-Typen einzeln berechnet. Diese Daten sind in Tabelle 5.1 aufgelistet und in Abbildung 5.3 graphisch dargestellt.

Wenn auch die Unterschiede zwischen den einzelnen AGN-Typen im Vergleich zur Streuung innerhalb eines Typs relativ gering sind, so deutet sich doch die Tendenz an, dass $\mathrm{f}_{\mathrm{X} / \mathrm{B}}$ mit abnehmendem Typ-Parameter mehr oder weniger kontinuierlich zunimmt. Diese Tendenz ordnet sich in logischer Weise in die von Maccacaro et al. (1988) gegebene Sequenz ein. Die in Abschnitt 3.3 eingeführte Typ-Sequenz von 0.1 (BL Lac) bis 3 (LINER) kann somit auch als Aktivitäts-Sequenz interpretiert werden.

Daraus ergibt sich für Seyfert-1, Seyfert-2 und LINER-Galaxien eine nicht überraschende Abnahme ihres Aktivitätsgrades in der genannten Reihenfolge.

Aus dem Emissionslinienspektrum kann man eine geringere Anregung der LINERGalaxien im Vergleich zu Seyfert-Galaxien ableiten; das entspricht dem hier postulierten geringerem Aktivitätsgrad. Seyfert-1 Galaxien kann man als aktiver als Seyfert-2 Galaxien bezeichnen, da man zusätzlich zur in beiden Galaxientypen ähnlichen Narrow-Line Region (NLR) in den Seyfert-1 Galaxien die Broad-Line Region beobachtet, die unter anderem auch einen erheblichen zusätzlichen Emissionslinienfluss produziert.

In diesem Zusammenhang werden nur die direkt beobachteten Eigenschaften ver- 


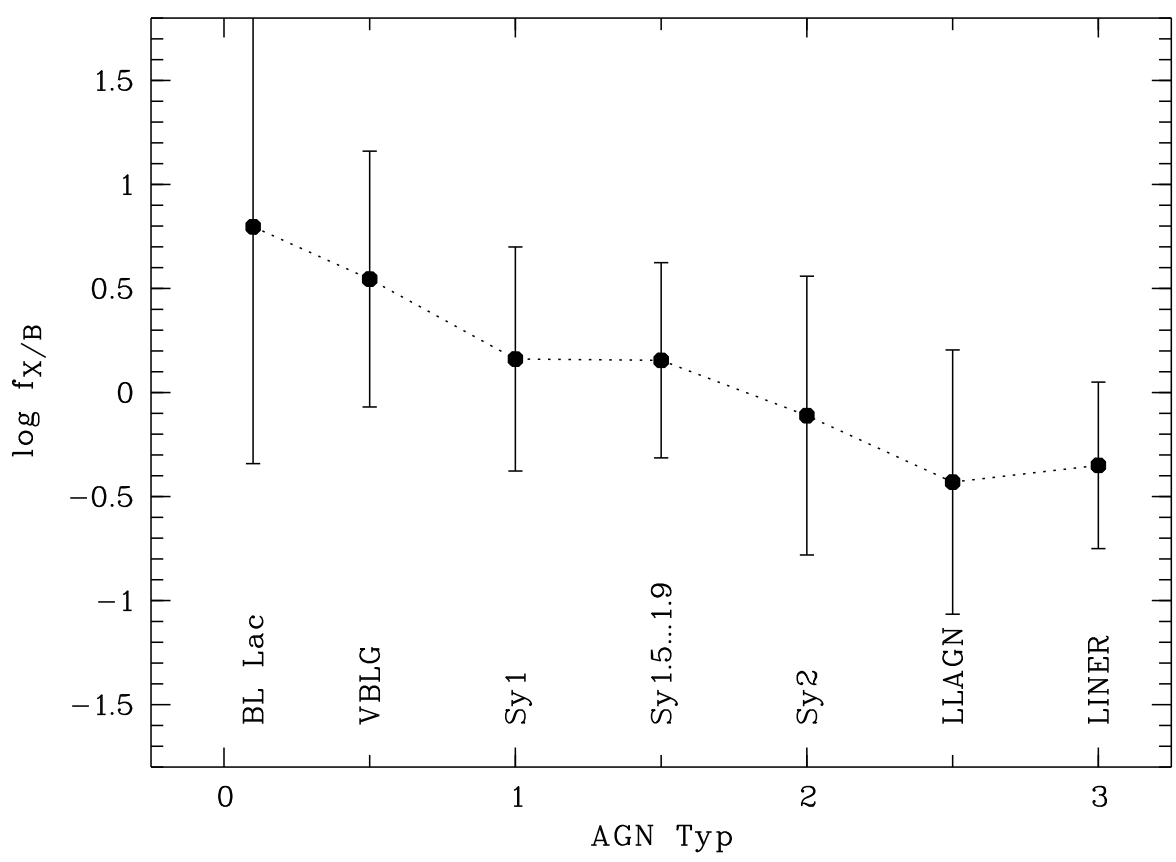

Abbildung 5.3: Das Flussverhältnis $f_{X / B}$ als Funktion des Aktivitätstyps. Trotz relativ großer statistischer Unsicherheiten ist ein Trend erkennbar, dass $\mathrm{f}_{X / \mathrm{B}}$ in der hier verwendeten Sequenz mit zunehmendem Typ-Parameter abnimmt. Damit kann diese zunächst rein formal eingeführte Typ-Sequenz auch als AktivitätsSequenz verstanden werden.

glichen, und es ist somit immer der beobachtete Aktivitätsgrad gemeint - selbst wenn alle Seyfert-Galaxien wie im Rahmen des Unified Model angenommen intrinsisch gleich aufgebaut sein sollten und damit auch intrinsisch den gleichen Aktivitätsgrad hätten, sind die direkt beobachteten Eigenschaften wie Emissionslinienspektrum und $\mathrm{f}_{\mathrm{X} / \mathrm{B}}$ unterschiedlich.

Verfolgt man diese Systematik weiter, ergibt sich für die VBLG, dass sie ein etwas höheres Maß an Aktivität als gewöhnliche Seyfert-1 Galaxien zeigen. Dies ist insofern ein wichtiger Punkt, da VBLG formal den Seyfert-1 Galaxien zuzurechnen sind.

\subsection{Die Ferninfrarot-Radio-Korrelation}

Eines der bemerkenswertesten Ergebnisse aus Untersuchungen extragalaktischer Quellen mit dem IRAS-Satelliten ist die enge Korrelation zwischen Ferninfrarot- und Radiokontinuumsemission in Galaxien (de Jong et al. 1985, van Driel et al. 1991, Niklas 1997, Heisler 1999, Thean et al. 2001). 
Bereits Helou et al. (1985) fanden für ein Sample aus 38 Scheibengalaxien eine sehr enge, lineare Korrelation dieser Größen. Diese Korrelation ist durch ein charakteristisches Verhältnis q bestimmt, dass die Autoren als

$$
q=\log \frac{f_{F I R} / 3.75 \cdot 10^{12} \mathrm{~Hz}}{f_{R}}
$$

definieren. Dabei entspricht die Frequenz $3.75 \cdot 10^{12} \mathrm{~Hz}$ der mittleren Wellenlänge von $80 \mu \mathrm{m}$ im FIR-Band, $\mathrm{f}_{R}$ ist der bei $1.4 \mathrm{GHz}$ gemessene Strahlungsfluss. Für ihr Gesamtsample errechneten sie einen mittleren Wert von $\langle\mathrm{q}\rangle=2.14$ mit einer sehr geringen Dispersion von 0.14 .

Besonders bemerkenswert an der von Helou et al. (1985) beschriebenen Korrelation ist, dass sie für verschiedenste Objekte gilt: von der ,ruhigen“ Scheibengalaxie M31 über Spiralgalaxien später Typen mit kontinuierlicher Sternentstehung bis hin zu Galaxien, die von einem nuklearen Starburst dominiert werden. In erster Näherung charakterisiert das beobachtete mittlere q die Sternentstehungsaktivität im Allgemeinen.

Helou et al. (1985) schätzten ab, dass weniger als $10 \%$ der Radiostrahlung bei $1.4 \mathrm{GHz}$ von Supernovaüberresten (SNR) stammt. Dieses Ergebnis stimmt überein mit Resultaten von Biermann (1976) und D'Odorico et al. (1982). Folglich wird die nichtthermische Radioemission aus Scheibengalaxien von der Synchrotronemission der Kosmischen Strahlung dominiert.

Die Aktiven Galaxien des Helou-Samples haben signifikant höhere FIR- und Radioleuchtkräfte als die nicht aktiven Galaxien. Andererseits weichen die Aktiven Galaxien nicht signifikant von der FIR-Radio-Korrelation ab. Das läßt darauf schließen, dass sowohl FIR- als auch Radioemission von der Sternentstehungsaktivität dominiert werden, die in den Aktiven Galaxien im Mittel stärker ist als in nicht aktiven Galaxien. Das hier vorliegende Sample ist aufgrund seiner Zusammensetzung besonders geeignet, diese Zusammenhänge genauer zu untersuchen.

In der Systematik der hier bisher benutzten Nomenklatur wird im Folgenden

$$
\log f_{F I R / R}=q
$$

benutzt. In Abbildung 5.4 ist links der Radiofluss gegen den FIR-Fluss aufgetragen. Die untere eingezeichnete Gerade spiegelt das mittlere Flussverhältnis $<q>$ von Helou et al. (1985) wieder. Man erkennt ein deutlich unterschiedliches Verhalten der beiden Untergruppen XAGN und VBLG: während die XAGN sehr gut der Korrelation von Helou et al. (1985) folgen, weichen die VBLG deutlich davon ab und passen besser zu der oberen Gerade, deren Definition weiter unten beschrieben wird. 

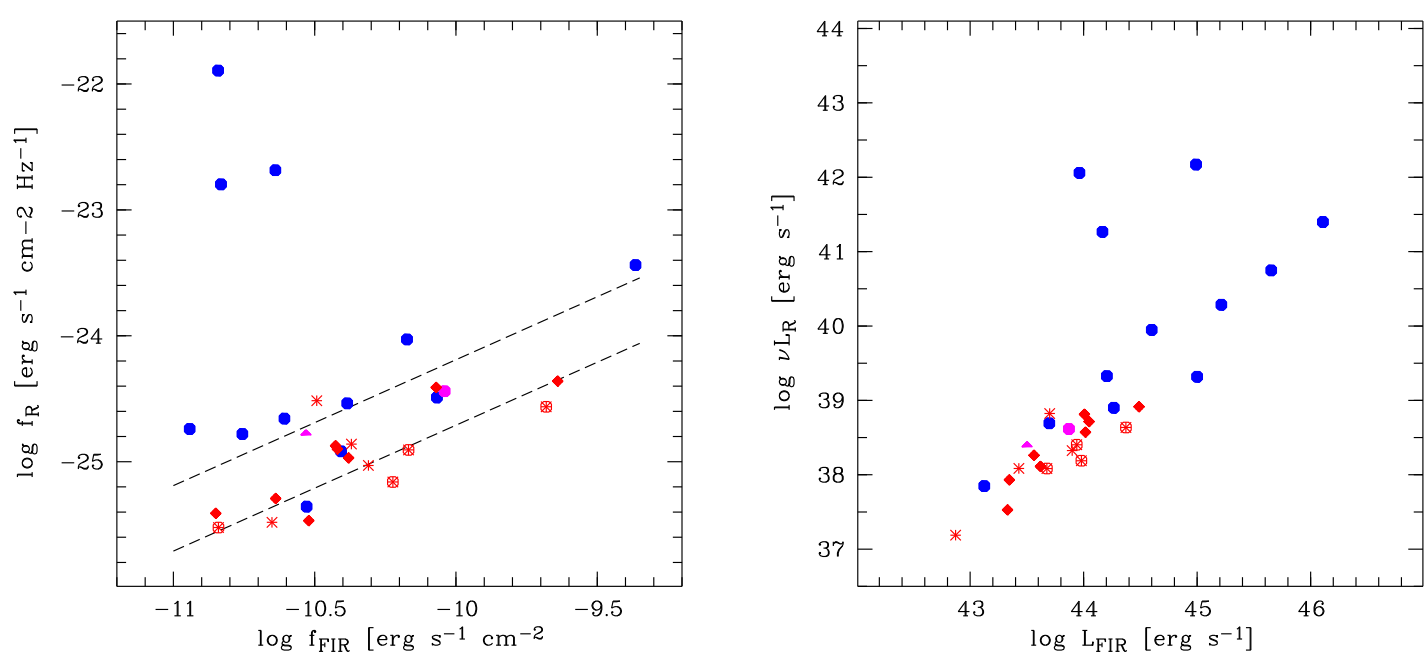

Abbildung 5.4: Links ist der Radiofluss gegen den FIR-Fluss aufgetragen (Ferninfrarot-Radio-Korrelation). Die untere Gerade spiegelt das von Helou et al. (1985) gefundene mittlere Flussverhältnis $<q>$ wieder, das auch sehr gut zum vorliegenden XAGN-Teilsample passt. Die obere Gerade entspricht dem geringeren $<q>$ für das VBLG*-Teilsample (s. Tabelle 5.2). Rechts ist zum Vergleich die Radioleuchtkraft gegen die FIR-Leuchtkraft aufgetragen. Drei VBLG heben sich durch hohen Radiofluss bzw. hohe Radioleuchtkraft deutlich von den übrigen Objekten ab $\left(\mathrm{f}_{R}>10^{-23} \mathrm{erg} \mathrm{s}^{-1} \mathrm{~cm}^{-2} \mathrm{~Hz}^{-1}\right)$; sie sind im VBLG*-Teilsample nicht enthalten und folglich auch nicht in die Berechnung der oberen Gerade eingegangen.

Im rechten Teil der Abbildung 5.4 ist zum Vergleich die Radioleuchtkraft gegen die FIR-Leuchtkraft aufgetragen.

Abbildung 5.5 zeigt das Flussverhältnis $\mathrm{f}_{\mathrm{FIR} / \mathrm{R}}$ sowohl als Funktion der FIR-Leuchtkraft wie auch der Radioleuchtkraft. In beiden Fällen sind die Verhältnisse $<q>$ der Abbildung 5.4 als konstante Geraden eingezeichnet. Wiederum ist leicht zu sehen, dass die XAGN sehr gut zu dem Helou-Wert von $<q>=2.14$ passen (obere Gerade), während die VBLG sehr viel stärker streuen und im Mittel ein niedrigeres $f_{F I R / R}$ aufweisen (untere Gerade).

Drei VBLG-Objekte heben sich durch einen besonders hohen Radiofluss und damit ein niedriges $\mathrm{f}_{\mathrm{FIR} / \mathrm{R}}$-Verhältnis stark von allen übrigen Quellen $\mathrm{ab}$. Sie sind sowohl in Abbildung $5.4\left(\mathrm{f}_{R}>10^{-23} \mathrm{erg} \mathrm{s}^{-1} \mathrm{~cm}^{-2} \mathrm{~Hz}^{-1}\right)$ als auch in Abbildung $5.5\left(\log \mathrm{f}_{\mathrm{FIR} / \mathrm{R}}\right.$ $<0)$ leicht zu identifizieren. Da hier offensichtlich eine zusätzlich Komponente in der Radioemission vorliegt, die den Radiofluss um etwa zwei Größenordnungen erhöht, liegt die Vermutung nahe, dass Radio-Jets die Ursache sein könnten.

Im Umkehrschluss ist mindestens ebenso bemerkenswert, dass im Vergleich dazu alle 

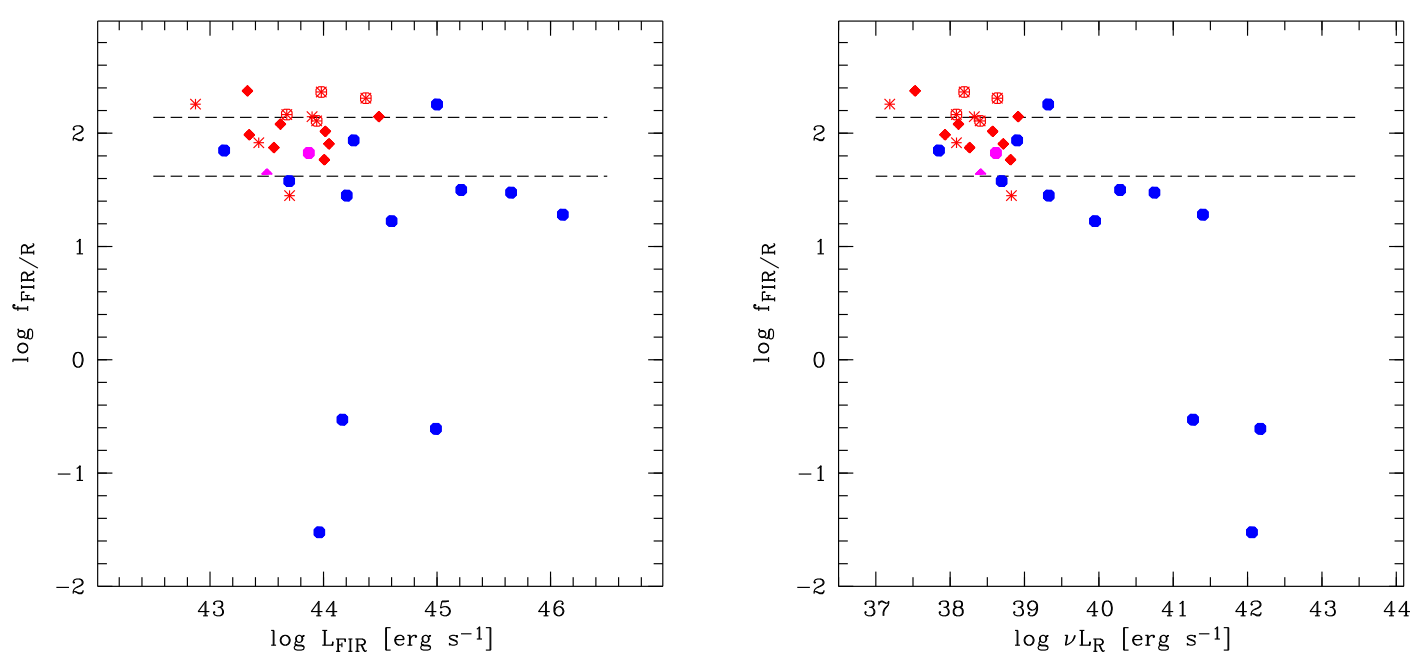

Abbildung 5.5: Das Flussverhältnis $\mathrm{f}_{\mathrm{FIR} / \mathrm{R}}$ als Funktion der FIR-Leuchtkraft $\mathrm{L}_{\mathrm{FIR}}$ bzw. der Radioleuchtkraft $v L_{R}$. Die obere Gerade gibt das von Helou et al. (1985) gefundene mittlere Flussverhältnis $<\mathrm{q}>=2.14$ wieder. Während die XAGN sehr gut mit diesem Wert vereinbar sind, weichen die VBLG teilweise stark davon $\mathrm{ab}$ und passen besser zur unteren Gerade, die dem mittleren $<\mathrm{q}>=1.62$ für die VBLG* entspricht (s. Tabelle 5.2).

anderen Quellen auch bezüglich ihrer Radioeigenschaften relativ dicht beieinander liegen und somit nicht durch besondere Radio-Strukturen wie Jets dominiert zu sein scheinen.

Die in Tabelle 5.2 wiedergegebenen statistischen Daten bestätigen dieses Ergebnis eindeutig: während der Mittelwert $<\log \mathrm{f}_{\mathrm{FIR} / \mathrm{R}}>$ für das XAGN-Sample im Rahmen der Genauigkeit mit dem Wert von Helou et al. (1985) übereinstimmt, weicht er für das VBLG-Sample stark davon ab. Die Streuung $\sigma$ ist für das VBLG-Sample sehr viel höher als für das XAGN- und das Helou-Sample.

Lässt man die drei stark abweichenden VBLG bei der Statistik unberücksichtigt, sinkt die Streuung erheblich von 1.19 auf 0.33 (Untersample VBLG* in Tabelle 5.2), liegt aber dennoch über derjenigen der XAGN und des Helou-Samples. Ebenso nähert sich das mittlere $\mathrm{f}_{\mathrm{FIR} / \mathrm{R}}$ den beiden anderen Samples, bleibt aber noch signifikant unter den entsprechenden Werten. Dem für dieses VBLG*-Sample berechneten $<\log \mathrm{f}_{\mathrm{FIR} / \mathrm{R}}>$ von 1.62 entspricht die obere Gerade der Abbildung 5.4 (links) und die untere Gerade der Abbildung 5.5.

Die drei abweichenden Quellen haben Flussverhältnisse $f_{F I R / R}$ zwischen -0.53 und -1.52 , der entsprechende Mittelwert für das Gesamtsample exklusive dieser Objekte (AGN*) beträgt hingegen $<\log \mathrm{f}_{\mathrm{FIR} / \mathrm{R}}>=1.88 \pm 0.33$. Damit liegt das $\mathrm{f}_{\mathrm{FIR} / \mathrm{R}}$ der drei Quellen um mehr als zwei bis drei Größenordnungen bzw. sieben bis zehn Standard- 
Tabelle 5.2: Statistik des Flussverhältnis $f_{F I R / R}$ für das Gesamtsample (AGN) und die Untergruppen XAGN und VBLG. Weiterhin sind die Samples AGN* und

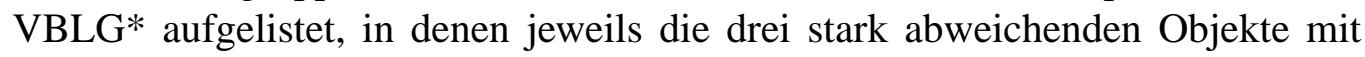
$\mathrm{f}_{\mathrm{FIR} / \mathrm{R}}<0$ fehlen (s. Abbildung 5.5). Zum Vergleich sind die Werte des Samples von Helou et al. (1985) mit angeführt.

\begin{tabular}{lcrccc}
\hline \hline & & \multicolumn{4}{c}{$\log \mathrm{f}_{\mathrm{FIR} / \mathrm{R}}$} \\
& $\mathrm{N}$ & Min. & Max. & $<\log \mathrm{f}_{\mathrm{FIR} / \mathrm{R}}>$ & $\sigma$ \\
\hline AGN & 30 & -1.52 & 2.37 & 1.61 & 0.92 \\
AGN & 27 & 1.22 & 2.37 & 1.88 & 0.33 \\
XAGN & 18 & 1.45 & 2.37 & 2.02 & 0.25 \\
VBLG & 12 & -1.52 & 2.25 & 0.99 & 1.19 \\
VBLG* & 9 & 1.22 & 2.25 & 1.62 & 0.33 \\
Helou et al. (1985) & 38 & & & 2.14 & 0.14 \\
\hline \hline
\end{tabular}

abweichungen unter dem AGN*-Mittelwert. Diese Quantifizierung soll nochmals die hohe Signifikanz der Abweichung dieser Quellen aufzeigen.

Weiter erhärtet werden die Ergebnisse der statistischen Analyse durch die Korrelationsanalyse, die äquivalente Resultate liefert: während die XAGN eine sehr starke Korrelation zwischen Radio- und FIR-Fluss zeigen $(\mathrm{r}=0.72, \mathrm{P}=0.001)$, sind die Größen nur für das VBLG*-Sample korreliert $(\mathrm{r}=0.72, \mathrm{P}=0.03)$, nicht jedoch für das VBLGSample inklusive der drei abweichenden Quellen $(r=-0.07, \mathrm{P}=0.83)$.

Zusammenfassend kann festgehalten werden, dass die XAGN trotz ihrer nuklearen Aktivität sehr gut mit der Ferninfrarot-Radio-Korrelation für nicht aktive Galaxien im Einklang stehen. Unter den VBLG befinden sich drei Quellen, die ein sehr viel kleineres Flussverhältnis $\mathrm{f}_{\mathrm{FIR} / \mathrm{R}}$ fernab von allen anderen Objekten zeigen und daher offensichtlich durch besondere Radioemission (möglicherweise Jets) dominiert werden. Die übrigen VBLG zeigen zwar auch eine FIR-Radio-Korrelation, allerdings mit einem niedrigeren mittleren $\mathrm{f}_{\mathrm{FIR} / \mathrm{R}}$ und größerer Streuung als die XAGN. Dies ist ein Indiz dafür, dass es intrinsische physikalische Unterschiede zwischen den XAGN (bzw. „normalen“ AGN) und den VBLG geben kann.

Bei diesen Betrachtungen ist zu beachten, dass im Vergleich zu den Datensätzen der anderen Frequenzbänder der $\mathrm{f}_{\mathrm{FIR} / \mathrm{R}}$-Datensatz relativ klein ist. Die Radioflüsse dieses Teils des Datensatzes stammen ausnahmslos aus dem NVSS und sind daher wie auch von Helou et al. (1985) bei $1.4 \mathrm{GHz}$ gemessen und somit direkt vergleichbar.

Um einen möglichen Zusammenhang von $\mathrm{f}_{\mathrm{FIR} / \mathrm{R}}$ mit der FIR-Farbe FIR $\mathrm{R}_{\text {col }}$ zu unter- 


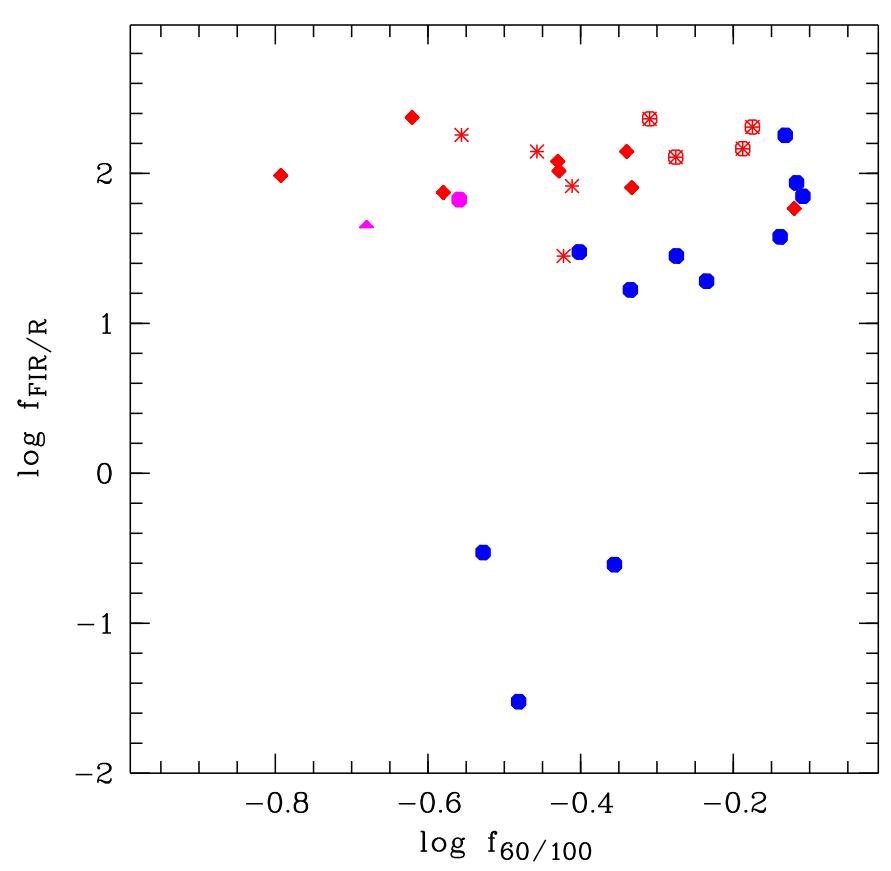

Abbildung 5.6: Das Flussverhältnis $f_{\mathrm{FIR} / \mathrm{R}}$ als Funktion der FIR-Farbe FIR $\mathrm{col}_{\text {. }}$ Es ist kein Zusammenhang zwischen beiden Größen erkennbar.

suchen, wurden diese Größen in Abbildung 5.6 gegeneinander aufgetragen. Der bereits in Abschnitt 4.6.3 beschriebene Effekt, dass die VBLG im Mittel etwas höhere $\mathrm{FIR}_{\mathrm{col}}$-Werte und damit wärmere Staubtemperaturen zeigen, ist hier noch auffälliger. Ein Zusammenhang mit $\mathrm{f}_{\mathrm{FIR} / \mathrm{R}}$ ist allerdings nicht zu erkennen.

\section{$5.3 \mathbf{f}_{\mathrm{X} / \mathrm{FIR}}$ als Maß für Sternentstehungsaktivität}

In der Folge von Sternentstehungsausbrüchen, sog. Starbursts, können massereiche Sterne und Röntgendoppelsterne (X-ray binaries XRB) eine erhebliche Röntgenleuchtkraft erzeugen. Daher stellte sich die Frage nach dem möglichen Beitrag von Starbursts zur Röntgenemission unserer Quellen. Um dieser Frage nachzugehen, vergleichen wir die Röntgenemission mit Ferninfrarot-Messungen des Satelliten IRAS.

In Abbildung 5.7 sind Röntgen- und FIR-Fluss bzw. Leuchtkraft jeweils gegeneinander aufgetragen. Zwischen den Flüssen ist kein Zusammenhang erkennbar, was auch durch die Korrelationsanalyse bestätigt wird $(\mathrm{r}=-0.14, \mathrm{P}=0.42)$. Die zum Vergleich gezeigten Leuchtkräfte sind hingegen aus den oben (s. Abschnitt 5.1) geschilderten Gründen deutlich korreliert $(\mathrm{r}=0.68, \mathrm{P}<0.001)$.

Das Verhältnis von Röntgen- zu Ferninfrarot-Fluss 

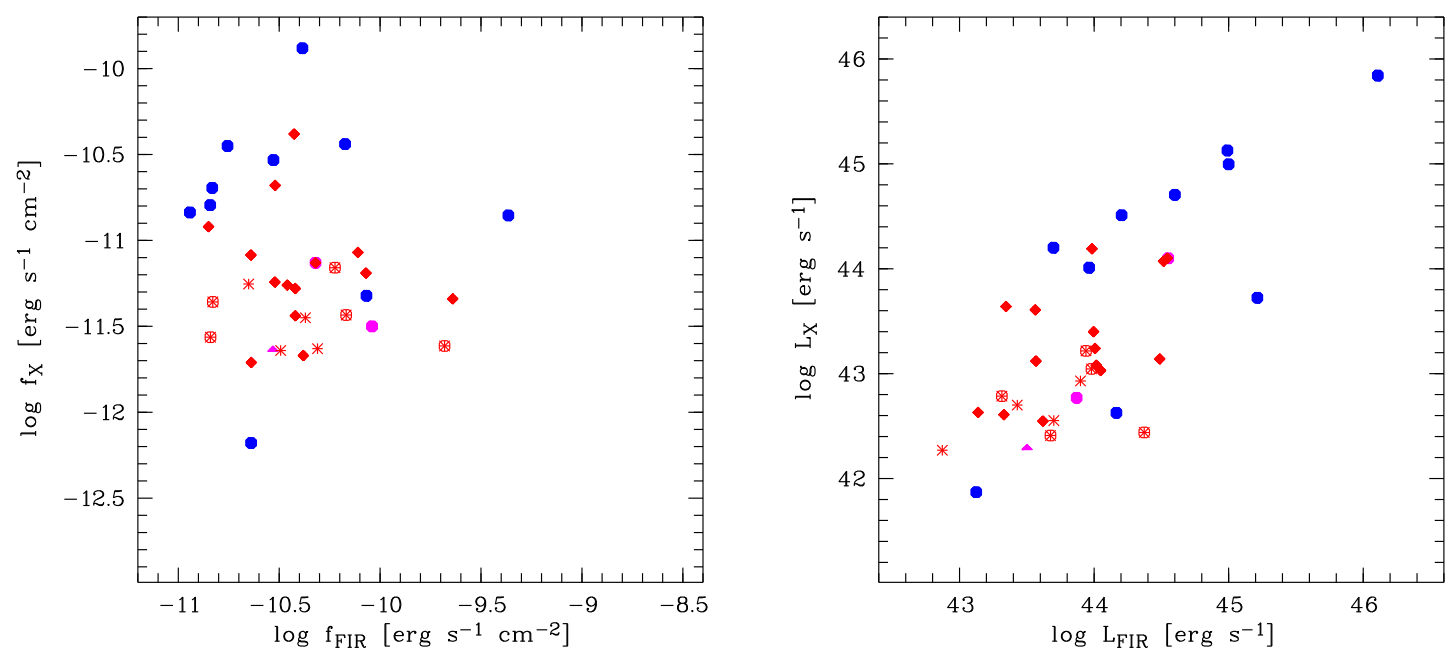

Abbildung 5.7: Röntgenfluss als Funktion des FIR-Flusses (links) bzw. Röntgenleuchtkraft als Funktion der FIR-Leuchtkraft (rechts).

$$
f_{X / F I R}=\frac{f_{X}}{f_{F I R}}
$$

ist wesentlich interessanter als die Einzelbetrachtung der Flüsse, da es der Bestimmung des Beitrags von Röntgenemission durch Sternentstehungsausbrüche dienen kann. Weil in guter Näherung sowohl der Röntgen- als auch der Ferninfrarot-Fluss ein lineares Maß für die Sternbildungsaktivität darstellen, ist das Verhältnis der beiden Größen etwa konstant, sofern der Starburst die Emission dominiert; Boller \& Bertoldi (1996) sowie Boller (1999) findet im Mittel Werte um $\mathrm{f}_{\mathrm{X} / \mathrm{FIR}}=0.003$ sowie obere und untere Grenzen von 0.01 bzw. 0.001 .

In Abbildung 5.8 ist dieses Verhältnis als Funktion von FIR- und Röntgenleuchtkraft aufgetragen. Es sind weiterhin der Mittelwert sowie Ober- und Untergrenze für $f_{X / F I R}$ von Boller (1999) als Geraden eingezeichnet. Da nahezu alle Objekte dieses Samples oberhalb der Geraden liegen, kann ihre Röntgenstrahlung nicht durch Sternentstehungsausbrüche dominiert sein. Man kann sogar folgern, dass für die meisten unserer Objekte der Starburst-Beitrag zur Röntgenemission keine wesentliche Rolle spielt, da sie deutlich über der Obergrenze von Boller (1999) liegen.

Im Mittel haben die Quellen unseres Samples mit $<\log \mathrm{f}_{\mathrm{X} / \mathrm{FIR}}>-0.8 \pm 0.7 \mathrm{bzw}$. $<\mathrm{f}_{\mathrm{X} / \mathrm{FIR}}>=0.15$ ein 50fach höheres Flussverhältnis $\mathrm{f}_{\mathrm{X} / \mathrm{FIR}}$ als die Starburst-Galaxien von Boller (1999).

Die Korrelationsanalyse bestätigt eindeutig den visuellen Eindruck der Abbildung 5.8: während es keine Korrelation zwischen $\mathrm{L}_{\mathrm{FIR}}$ und $\mathrm{f}_{\mathrm{X} / \mathrm{FIR}}$ gibt $(\mathrm{r}=0.07, \mathrm{P}=0.71)$, existiert eine äußerst signifikante Korrelation zwischen $\mathrm{L}_{X}$ und $\mathrm{f}_{X / F I R}(r=0.68, P<0.001)$. 

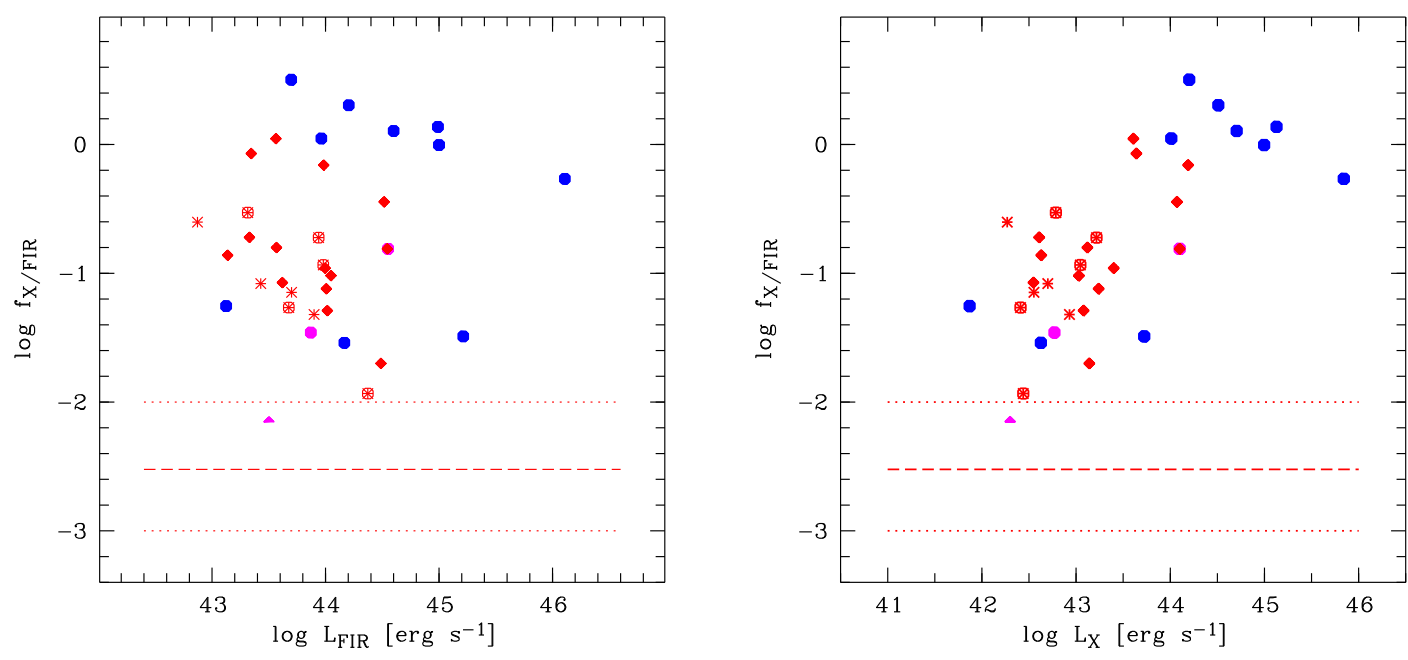

Abbildung 5.8: Verhältnis von Röntgen- zu Ferninfrarot-Fluss $f_{X / F I R}$ als Funktion von FIR-Leuchtkraft (links) und Röntgenleuchtkraft (rechts). Als Geraden eingezeichnet sind Mittelwert sowie Ober- und Untergrenze für ein vom StarburstEmission bestimmtes $f_{X / F I R}$ (Boller 1999). Nahezu alle Galaxien dieses Samples liegen über der Obergrenze und können daher bzgl. $f_{X / F I R}$ nicht von StarburstEmission dominiert sein.

Daraus kann man folgern, dass das Verhältnis $f_{X / F I R}$ von der Röntgenemission der Quellen bestimmt wird.

Beide Beobachtungen - der starke Exzess von $\mathrm{f}_{\mathrm{X} / \mathrm{FIR}}$ gegenüber Starburst-Emission und die Korrelation von $\mathrm{f}_{\mathrm{X} / \mathrm{FIR}}$ mit der Röntgenleuchtkraft - stützen die Hypothese, dass die Röntgenemission unserer Quellen von einem Aktiven Kern dominiert wird und sich dabei über einen relativ großen Bereich von fast fünf Größenordnungen erstreckt $\left(\mathrm{L}_{X}=\right.$ $10^{41.5} \ldots 10^{46} \mathrm{erg} \mathrm{s}^{-1}$ ), während die FIR-Leuchtkraft nicht dem Kern, sondern der unterliegenden Galaxie zuzuordnen ist und im Wesentlichen nur über zwei Größenordnungen streut $\left(\mathrm{L}_{\mathrm{FIR}}=10^{43} \ldots 10^{45} \mathrm{erg} \mathrm{s}^{-1}\right)$.

Ein Zusammenhang zwischen dem Aktivitätstyp und dem Flussverhältnis $f_{X / F I R}$ kann nicht sicher festgestellt werden. Die entsprechenden statistischen Werte sind in Tabelle 5.3 aufgelistet und in Abbildung 5.9 graphisch dargestellt. Die gegenüber den anderen Typen etwas höheren $\mathrm{f}_{\mathrm{X} / \text { FIR }}$-Werte für Seyfert-1 Galaxien und insbesondere für VBLG kann man zwar angesichts der errechneten Unsicherheiten nicht als signifikant bezeichnen, sie entsprechen aber tendenziell dem Gang von $\mathrm{f}_{\mathrm{X} / \mathrm{B}}$ mit dem Aktivitäts-Typ (s. Abbildung 5.3) und sind in diesem Zusammenhang evidenter als nur für sich betrachtet.

Ein Anstieg von $f_{X / F I R}$ mit abnehmenden Aktivitätstyp - parallel zum gleichen für $\mathrm{f}_{\mathrm{X} / \mathrm{B}}$ gefundenen Trend - wäre konsistent mit dem obigem Ergebnis, dass die Röntgen- 
Tabelle 5.3: Statistik des Flussverhältnis $f_{X / F I R}$ für das Gesamtsample (AGN) und verschiedene Untergruppen. Die wichtigsten Werte dieser Tabelle sind in Abbildung 5.9 graphisch dargestellt.

\begin{tabular}{lccccc}
\hline \hline & & \multicolumn{4}{c}{$\log \mathrm{f}_{\mathrm{X} / \mathrm{FIR}}$} \\
& $\mathrm{N}$ & Min. & Max. & $<\log \mathrm{f}_{\mathrm{X} / \mathrm{FIR}}>$ & $\sigma$ \\
\hline AGN & 37 & -2.59 & 0.50 & -0.83 & 0.70 \\
AGN-1 & 30 & -2.59 & 0.50 & -0.74 & 0.72 \\
VBLG & 11 & -2.59 & 0.50 & -0.55 & 1.00 \\
XAGN & 26 & -2.13 & 0.04 & -0.95 & 0.52 \\
XAGN-1 & 19 & -1.93 & 0.04 & -0.86 & 0.50 \\
BL Lac & 0 & & & & \\
Sy1 & 14 & -1.70 & 0.04 & -0.78 & 0.48 \\
Sy1.5...1.9 & 5 & -1.93 & -0.52 & -1.07 & 0.55 \\
Sy2 & 4 & -1.32 & -0.60 & -1.03 & 0.30 \\
LLAGN & 1 & -2.13 & -2.13 & -2.13 & \\
LINER & 2 & -1.46 & -0.81 & -1.13 & 0.46 \\
\hline \hline
\end{tabular}

emission überwiegend aus dem Aktiven Kern, die FIR-Emission hingegen aus der stellaren Komponente der Galaxie stammt, wobei eine Rückkopplung dieser beiden Komponenten, die den beschriebenen Trend glätten sollte, keinesfalls ausgeschlossen werden kann.

\section{$5.4 L_{F I R} / L_{B}$ als Maß der Sternentstehungsaktivität}

Das Verhältnis von Ferninfrarotleuchtkraft zu Blauleuchtkraft $L_{F I R} / L_{B}$ ist ein Maß für den Infrarotexzeß in Galaxien. Man kann dieses Verhältnis in einer ersten Näherung auch als direktes Maß für die Sternentstehungsaktivität betrachten. Während $\mathrm{L}_{\text {FIR }}$ überwiegend durch sehr junge, massereiche Sterne bestimmt wird, die sich noch in den Staubwolken befinden, in denen sie entstanden, spiegelt $\mathrm{L}_{\mathrm{B}}$ vor allem den Anteil an OB-Sternen wider. Aus diesem Grunde wird $\mathrm{L}_{\mathrm{FIR}} / \mathrm{L}_{\mathrm{B}}$ als Maß für das Verhältnis gegenwärtiger zu vergangener Sternentstehung betrachtet: ein hoher Wert deutet auf eine derzeit überdurchschnittliche Sternentstehung bis zum Starburst hin, ein niedriger Wert indiziert eine kontinuierliche Sternentstehung (Keel 1993, van Driel \& de Jong 1990, Klaas 1988). 


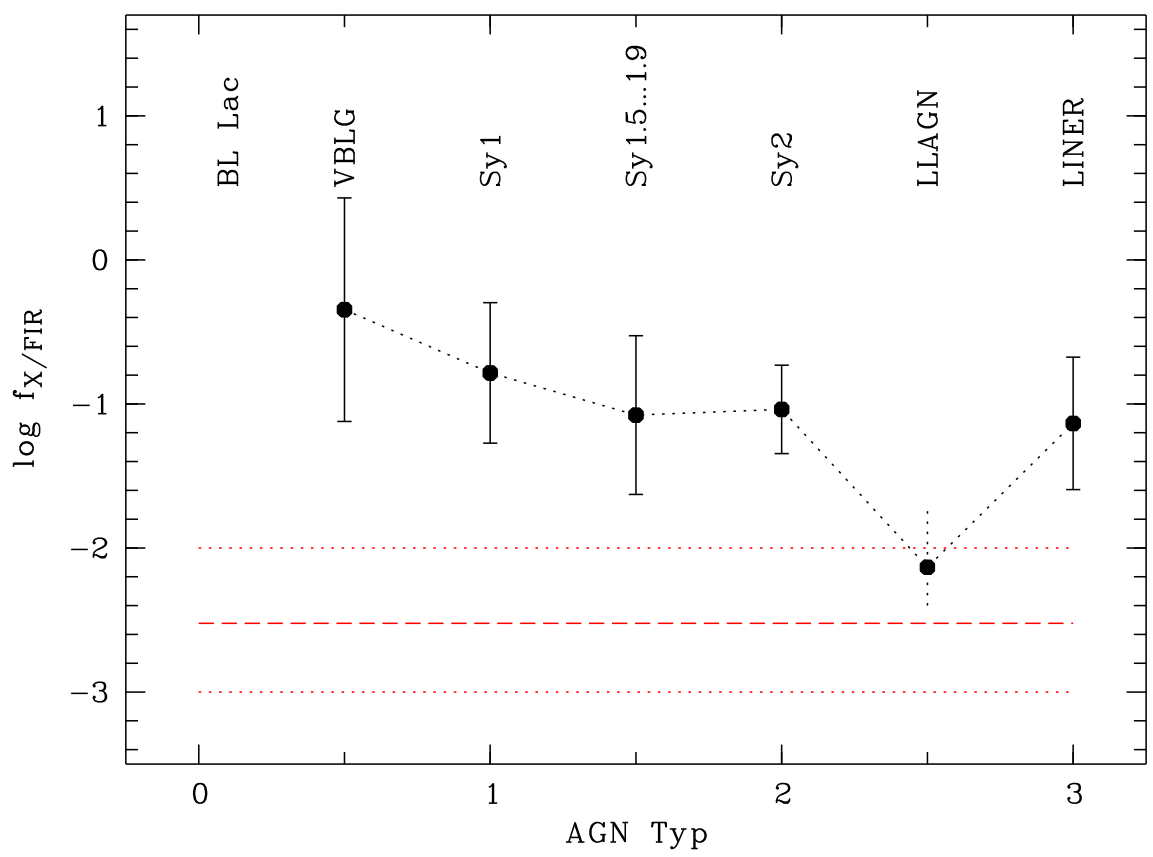

Abbildung 5.9: Das Flussverhältnis $f_{X / F I R}$ als Funktion des Aktivitätstyps. Wie in Abbildung 5.8 sind zusätzlich die Grenzen für den Beitrag von Starburst-Emission als Geraden eingezeichnet; man kann erkennen, dass auch für die einzelnen Aktivitätstypen (abgesehen von dem statistisch nicht relevanten einzelnen LLAGN) der Starburst-Beitrag zur Röntgenemission unbedeutend ist. Berücksichtigt man die ebenfalls eingezeichneten Standardabweichungen innerhalb der einzelnen Aktivitätstypen, ist ein Trend von $\mathrm{f}_{\mathrm{X} / \mathrm{FIR}}$ nicht sicher erkennbar.

Abbildung 5.10 zeigt den FIR-Fluss aufgetragen gegen die scheinbare optische Helligkeit $\mathrm{m}_{B}$ sowie die FIR-Leuchtkraft gegen die absolute optische Helligkeit $\mathrm{M}_{\mathrm{B}}$. Erneut sind die beiden absoluten Größen $\mathrm{L}_{\mathrm{FIR}}$ und $\mathrm{M}_{\mathrm{B}}$ sehr stark korreliert $(\mathrm{r}=-0.48$, $\mathrm{P}=0.002$ ), aber auch $\mathrm{f}_{\mathrm{FIR}}$ und $\mathrm{m}_{\mathrm{B}}$ zeigen eine nur geringfügig schwächere Korrelation $(\mathrm{r}=-0.43, \mathrm{P}=0.006)$. Dies untermauert den oben beschriebenen Zusammenhang und bestätigt, dass auch für das hier vorliegende Sample die Sternentstehungsaktivität einen bestimmenden Einfluss auf den ferninfraroten und den optischen Fluss der Quellen hat.

Um die bisher benutzte Nomenklatur fortzuführen, wird die in der Literatur auch als Leuchtkraftverhältnis $\mathrm{L}_{\mathrm{FIR}} / \mathrm{L}_{B}$ bezeichnete Größe hier van Driel \& de Jong (1990) sowie Keel (1993) folgend als

$$
\log f_{F I R / B}=\log \frac{L_{F I R}}{L_{B}}=0.4 m_{B}+\log f_{F I R}+4.51
$$



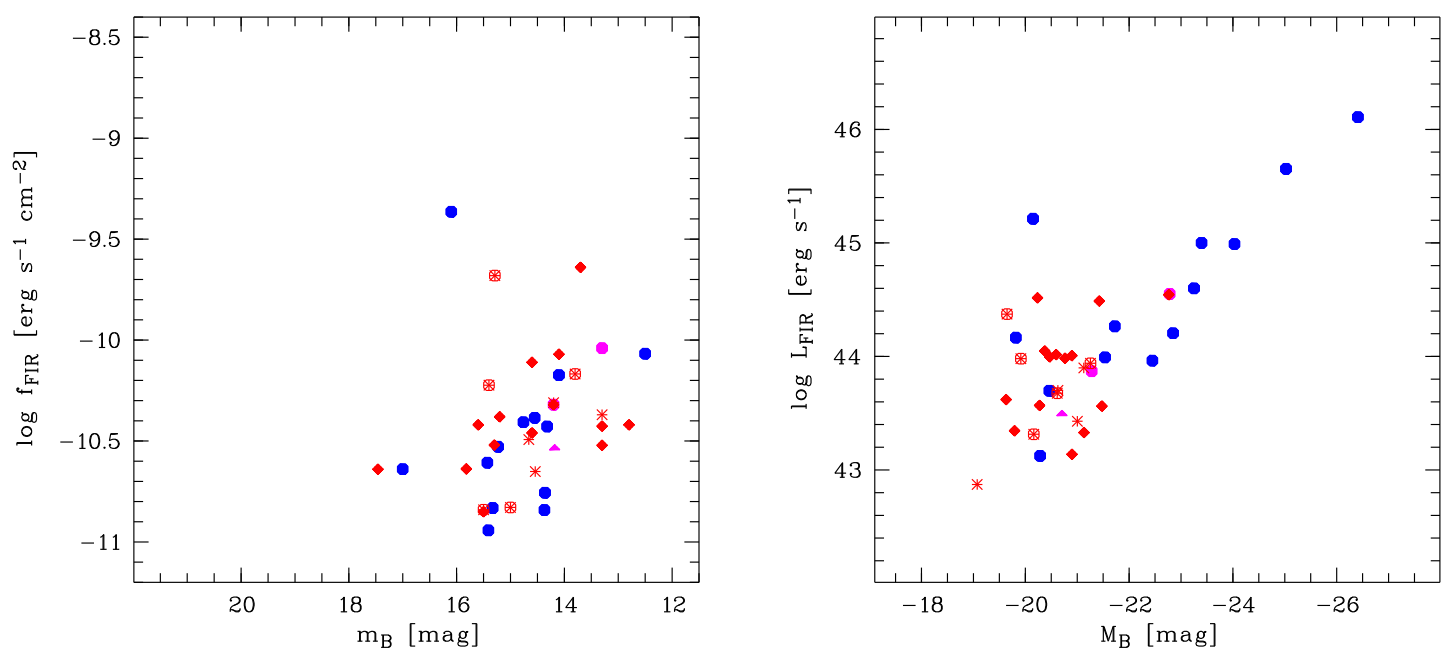

Abbildung 5.10: FIR-Fluss $\mathrm{f}_{\mathrm{FIR}}$ gegen die scheinbare optische Helligkeit $\mathrm{m}_{B}$ (links) bzw. FIR-Leuchtkraft LFIR gegen die absolute optische Helligkeit $\mathrm{M}_{\mathrm{B}}$ (rechts). Sowohl die beiden Flüsse als auch die beiden Leuchtkräfte sind jeweils untereinander signifikant korreliert (s. Text).

berechnet, wobei $f_{\text {FIR }}$ der durch Gleichung 4.9 gegebene FIR-Fluss ist.

In Abbildung 5.11 ist dieses Verhältnis als Funktion der FIR-Leuchtkraft dargestellt. Was schon in der linken Auftragung mit allen vorhandenen Daten erkennbar ist, wird bei getrennter Darstellung der Untergruppen XAGN und VBLG noch deutlicher: die beiden Untergruppen sind in diesem Diagramm deutlich zu unterscheiden.

Zur Quantifizierung dieses Effektes wurde sowohl eine Korrelationsanalyse als auch eine lineare Regression durchgeführt, deren Resultate in Tabelle 5.4 aufgelistet sind. In Abbildung 5.11 (unten rechts) erkennt man unter den VBLG zwei Quellen, die sich sehr deutlich von allen anderen abheben $\left(\log \mathrm{f}_{\mathrm{FIR} / \mathrm{B}}>0.5\right)$. Es handelt sich dabei um 3C390.3 und ESO 548- G 081. Die VBLG exklusive dieser beiden wurden für die statistische und die Korrelationsanalyse als neue Untergruppe $\mathrm{VBLG}^{+}$neben dem Gesamtsample (AGN) sowie den XAGN und VBLG separat betrachtet.

Für alle vier Samples ergeben sich signifikante Korrelationen zwischen $\mathrm{f}_{\mathrm{FIR} / \mathrm{B}}$ und $\mathrm{L}_{\mathrm{FIR}}$. Wie schon anhand der Abbildung 5.11 zu vermuten war, zeigen die beiden Teilsamples XAGN und VBLG eine stärkere Korrelation als das AGN Gesamtsample. Der Ausschluss der zwei stark abweichenden Quellen erhöht die Korrelation für $\mathrm{VBLG}^{+}$gegenüber VBLG nochmals deutlich von 0.59 auf 0.69 .

Ein analoges Bild liefert die Regressionsanalyse: Die Steigungen der Regressionsgeraden für die XAGN $(\mathrm{m}=0.65 \pm 0.14)$ und die $\mathrm{VBLG}^{+}(\mathrm{m}=0.18 \pm 0.07)$ sind signifikant unterschiedlich. Für das AGN Gesamtsample erhält man naturgemäß einen mittleren 

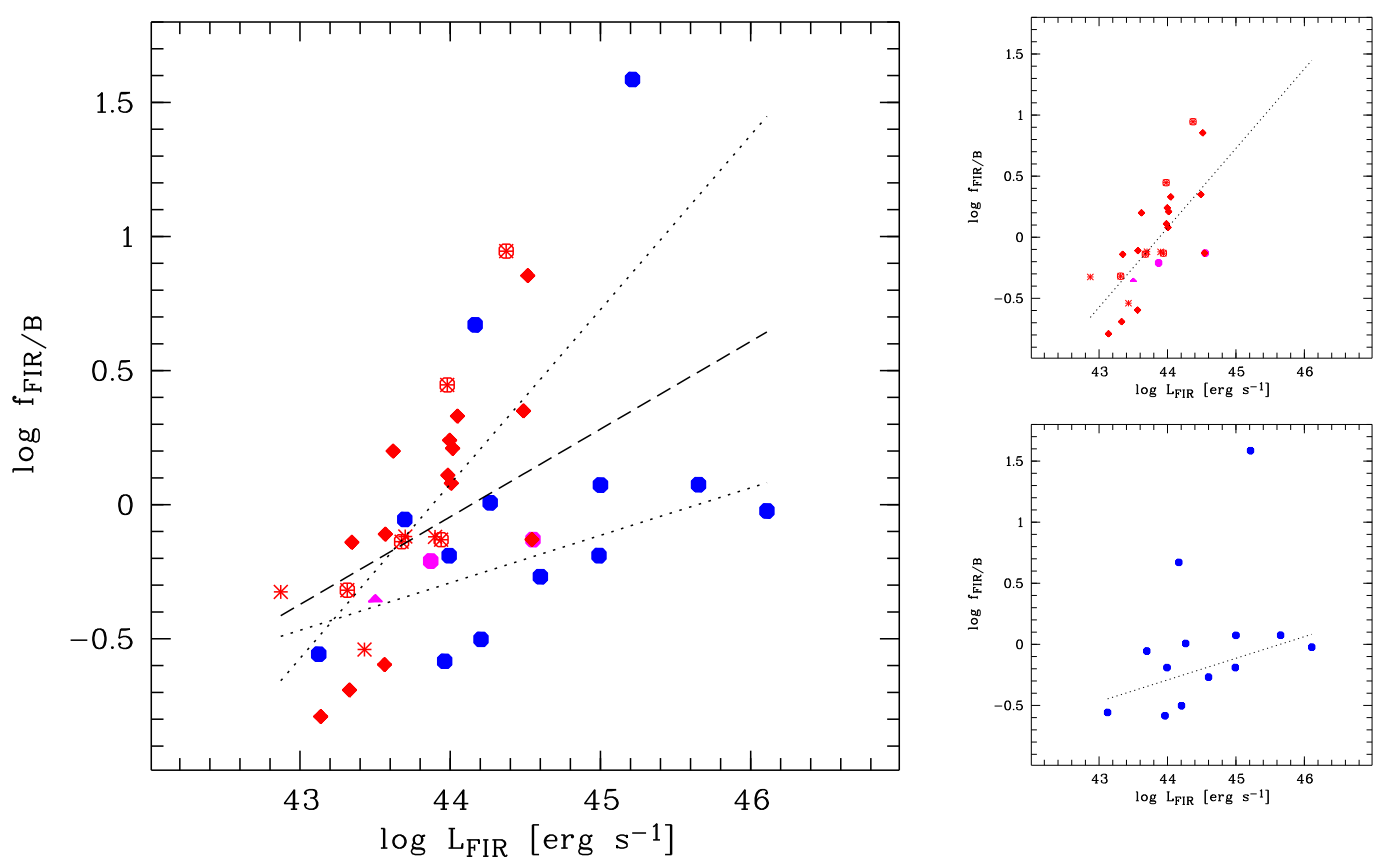

Abbildung 5.11: Fluss- bzw. Leuchtkraftverhältnis $\mathrm{f}_{\mathrm{FIR} / \mathrm{B}}$ als Funktion der FIRLeuchtkraft (links). Zur besseren Übersichtlichkeit sind rechts in gleicher Weise die Untergruppen XAGN (oben) und VBLG (unten) getrennt dargestellt. Zusätzlich sind die Regressionsgeraden für das AGN Gesamtsample (gestrichelt) sowie die Untergruppen XAGN und VBLG $^{+}$(gepunktet) eingezeichnet.

Wert.

Die Tatsache, dass eine Korrelation zwischen $\mathrm{f}_{\mathrm{FIR} / \mathrm{B}}$ und $\mathrm{L}_{\mathrm{FIR}}$ besteht, ist nicht überraschend. Ein entsprechendes Ergebnis wurde z.B. von Lehnert \& Heckman (1995) für ein Sample von etwa 50 Starburst-Galaxien publiziert. Wie oben bereits beschrieben führt ein junger Sternentstehungsausbruch zu starker Ferninfrarot-Emission und damit auch zu einem hohen Flussverhältnis $\mathrm{f}_{\mathrm{FIR} / \mathrm{B}}$, da die optische Emission erst mit zeitlicher Verzögerung ansteigt.

Sehr bemerkenswert hingegen ist, dass die Korrelation für die Untergruppen XAGN und $\mathrm{VBLG}^{+}$deutlich stärker ist als für das AGN Gesamtsample und sich für diese beiden Untergruppen signifikant unterschiedliche Regressionsgeraden errechnen. Ursache dafür könnte sein, dass nicht allein ein überall gleicher Starburst-Mechanismus in jeweils unterschiedlicher Stärke wirkt, sondern die Objekte der beiden Untergruppen zusätzlich intrinsische Unterschiede aufweisen. Es kann allerdings auch nicht ausgeschlossen werden, dass ein anderer Parameter als der AGN-Typ für die Zweiteilung der in Abbildung 5.11 dargestellten Daten verantwortlich ist. 
Tabelle 5.4: Ergebnisse der linearen Regression (Geradensteigung $m$ und Ordinatenabschnitt b) und der Korrelationsanalyse von $\mathrm{f}_{\mathrm{FIR} / \mathrm{B}}$ mit $\mathrm{L}_{\mathrm{FIR}}$. Die Untergruppe $\mathrm{VBLG}^{+}$entspricht der Untergruppe VBLG exklusive zweier deutlich abweichender Quellen (s. Text).

\begin{tabular}{lccccc}
\hline \hline & & \multicolumn{2}{c}{$\mathrm{f}_{\mathrm{FIR} / \mathrm{B}}-\mathrm{L}_{\mathrm{FIR}}$} & & \\
& $\mathrm{N}$ & $\mathrm{m}$ & $\mathrm{b}$ & $\mathrm{r}$ & $\mathrm{P}$ \\
\hline $\mathrm{AGN}$ & 39 & $0.34 \pm 0.10$ & $-14.4 \pm 4.4$ & 0.57 & $<0.001$ \\
$\mathrm{XAGN}$ & 26 & $0.65 \pm 0.14$ & $-28.5 \pm 6.0$ & 0.76 & $<0.001$ \\
VBLG & 13 & $0.27 \pm 0.19$ & $-12.1 \pm 8.6$ & 0.59 & 0.033 \\
$\mathrm{VBLG}^{+}$ & 11 & $0.18 \pm 0.07$ & $-8.1 \pm 3.3$ & 0.69 & 0.019 \\
\hline \hline
\end{tabular}

Die statistische Analyse des Flussverhältnisses $\mathrm{f}_{\mathrm{FIR} / \mathrm{B}}$ hinsichtlich der verschiedenen AGN-Typen ergab keine zusätzlichen Erkenntnisse: im Rahmen der Genauigkeiten ist das mittlere $\mathrm{f}_{\mathrm{FIR} / \mathrm{B}}$ für jeweils alle AGN-Typen mit dem Mittelwert für das Gesamtsample von $\left\langle\mathrm{f}_{\mathrm{FIR} / \mathrm{B}}\right\rangle=-0.026 \pm 0.472$ vereinbar, weshalb auf eine graphische Darstellung verzichtet wird.

In der Literatur wurde von einigen Autoren ein Zusammenhang zwischen $\mathrm{f}_{\mathrm{FIR} / \mathrm{B}}$ und der FIR-Farbe FIR $_{\text {col }}$ diskutiert, die man wie in Abschnitt 4.6.3 beschrieben als Maß für die Staubtemperatur betrachten kann. Klaas (1988) bzw. de Jong et al. (1984) fanden eine Korrelation dieser Größen für ein Sample von 89 Galaxien. Auch Lehnert \& Heckman (1995) fanden eine entsprechende Korrelation mit höherer Streuung als Klaas (1988), die allerdings nicht quantifiziert wird. Wolstencroft et al. (1986) zeigen eine entsprechende Abbildung für 94 Galaxien aus einem Feld um den südgalaktischen Pol, aus der ebenfalls eine Korrelation zwischen $\mathrm{f}_{\mathrm{FIR} / \mathrm{B}}$ und FIR $\mathrm{Fol}_{\mathrm{col}}$ ersichtlich ist. Bei van Driel \& de Jong (1990) ist hingegen für ein Sample von 49 S0-Galaxien bestenfalls eine marginale Korrelation erkennbar.

Abbildung 5.12 zeigt das Flussverhältnis $\mathrm{f}_{\mathrm{FIR} / \mathrm{B}}$ als Funktion der FIR-Farbe FIR col $_{\text {für }}$ das hier vorliegende Sample. Zusätzlich ist als gestrichelte Gerade die von Klaas (1988) beschriebene Korrelation eingezeichnet. Die Galaxien des hier vorliegenden AGNSamples befinden sich im gleichen Bereich des Diagramms wie bei Klaas (1988), weisen allerdings eine höhere Streuung auf. Ein Unterschied zwischen XAGN und VBLG ist in dieser Abbildung nicht zu erkennen. Es liegt keine signifikante Korrelation zwischen $\mathrm{f}_{\mathrm{FIR} / \mathrm{B}}$ und FIR col vor $(\mathrm{r}=0.15, \mathrm{P}=0.35)$. 


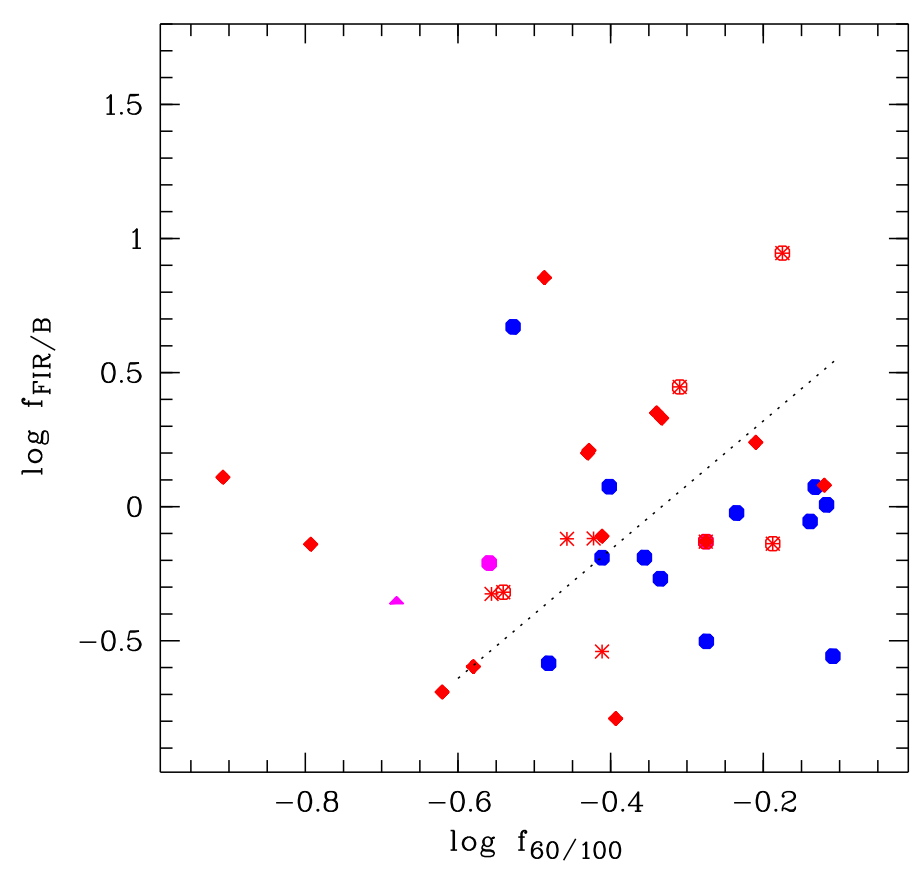

Abbildung 5.12: Das Flussverhältnis $\mathrm{f}_{\mathrm{FIR} / \mathrm{B}}$ als Funktion der Ferninfrarot-Farbe $\mathrm{FIR}_{\mathrm{col}}$. Die gepunktete Gerade spiegelt eine für ein Sample von 89 Galaxien gefundene Korrelation wider (Klaas (1988) bzw. de Jong et al. (1984)). Die Quellen unseres Samples liegen im gleichen Bereich, zeigen aber selbst keine signifikante Korrelation.

\section{$5.5 f_{R / B}$ als Kriterium für radiolaute und radioleise AGN}

Analog zu den bisherigen Abschnitten ist in Abbildung 5.13 der Radiofluss $\mathrm{f}_{R}$ gegen die scheinbare optische Helligkeit $m_{B}$ bzw. die Radioleuchtkraft $v L_{R}$ gegen die absolute optische Helligkeit $\mathrm{M}_{\mathrm{B}}$ aufgetragen. Beim Vergleich der scheinbaren Größen fällt auf, dass die VBLG im Radiobereich deutlich heller als die XAGN sind, im Optischen aber kein großer Unterschied besteht. Erneut zeigen die absoluten Größen $v L_{R}$ und $M_{B}$ ein sehr starke Korrelation $(\mathrm{r}=-0.68, \mathrm{P}<0.001)$, aber auch die scheinbaren Größen $\mathrm{f}_{R}$ und $\mathrm{m}_{\mathrm{B}}$ sind signifikant, wenn auch deutlich schwächer korreliert $(\mathrm{r}=0.24, \mathrm{P}=0.03)$.

Für den Vergleich von optischer und Radiohelligkeit verwendet man aus historischen Gründen die Begriffe radiolaut und radioleise. Während man zu Beginn der Radioastronomie die im Radiobereich detektierbaren Quellen als radiolaut und alle anderen als radioleise bezeichnete, stellte sich schnell heraus, dass die Emission extragalaktischer Quellen (ebenso wie anderer) auch im Radiobereich eine kontinuierlich Verteilung aufweist. Daher wird heute zur Unterscheidung von radiolauten und radioleisen Quellen das Verhältnis von Radio- zu optischem Fluss benutzt, das in der Literatur häufig mit R 

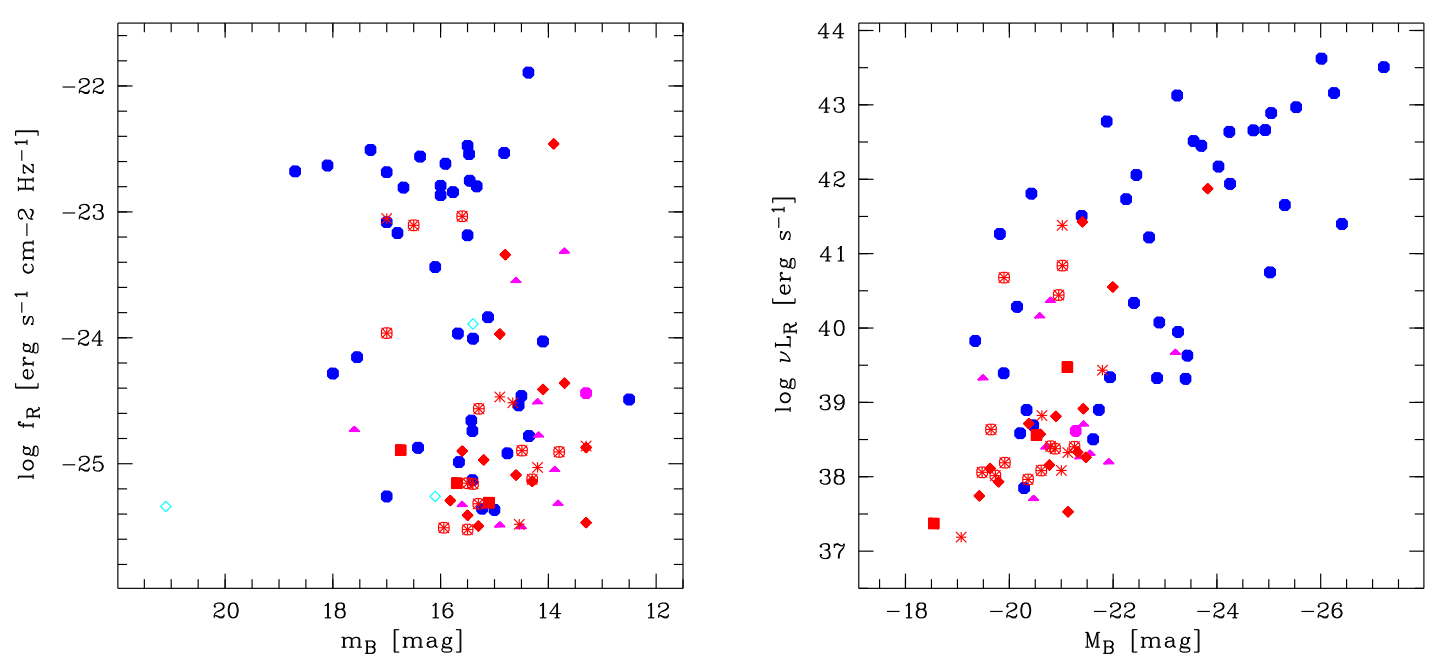

Abbildung 5.13: Radiofluss $\mathrm{f}_{R}$ gegen scheinbare optische Helligkeit $\mathrm{m}_{\mathrm{B}}$ (links) bzw. Radioleuchtkraft $\nu \mathrm{L}_{\mathrm{R}}$ gegen absolute optische Helligkeit $\mathrm{M}_{\mathrm{B}}$ (rechts).

bezeichnet wird. Mit der von Allen (1976) angegebenen Umrechnung der B-Magnitude in einen monochromatischen Fluss $f_{v}$ ergibt sich

$$
\log f_{R / B}=R=\log \frac{f_{R}}{f_{v, B}}=\log f_{R}+0.4 * m_{B}+19.36
$$

Ferner wird folgende Definition von Kellermann et al. (1989) übernommen:

$$
\begin{array}{llll}
\text { radioleise }(R Q): & f_{R / B}<1 & \text { bzw. } & \log f_{R / B}<0 \\
\text { radiolaut }(R L): & f_{R / B}>10 & \text { bzw. } & \log f_{R / B}>1
\end{array} .
$$

Dabei wird der Bereich $1<\mathrm{f}_{\mathrm{R} / \mathrm{B}}<10$ als Übergangsbereich angesehen; die entsprechenden Objekte werden gelegentlich als ,radio-intermediate (RI)“ bezeichnet (Miller et al. 1993, Falcke et al. 1996).

Abbildung 5.14 zeigt $f_{R / B}$ sowohl als Funktion der optischen Absoluthelligkeit $M_{B}$ als auch der Radioleuchtkraft $v L_{R}$. Man kann deutlich erkennen, dass $f_{R / B}$ kontinuierlich verteilt ist und daher eine definitorische Abgrenzung von radiolauten und -leisen Quellen notwendig ist. Die zu diesem Zweck oben angegebenen Grenzen von Kellermann et al. (1989) sind gestrichelt eingezeichnet.

Weiterhin erkennt man, dass die VBLG im Mittel ein höheres Flussverhältnis $\mathrm{f}_{\mathrm{R} / \mathrm{B}}$ aufweisen. Um dies zu quantifizieren wurden die Häufigkeiten von radiolauten und radioleisen Quellen für die Untergruppen VBLG, XAGN und XAGN-1 wie auch für die 

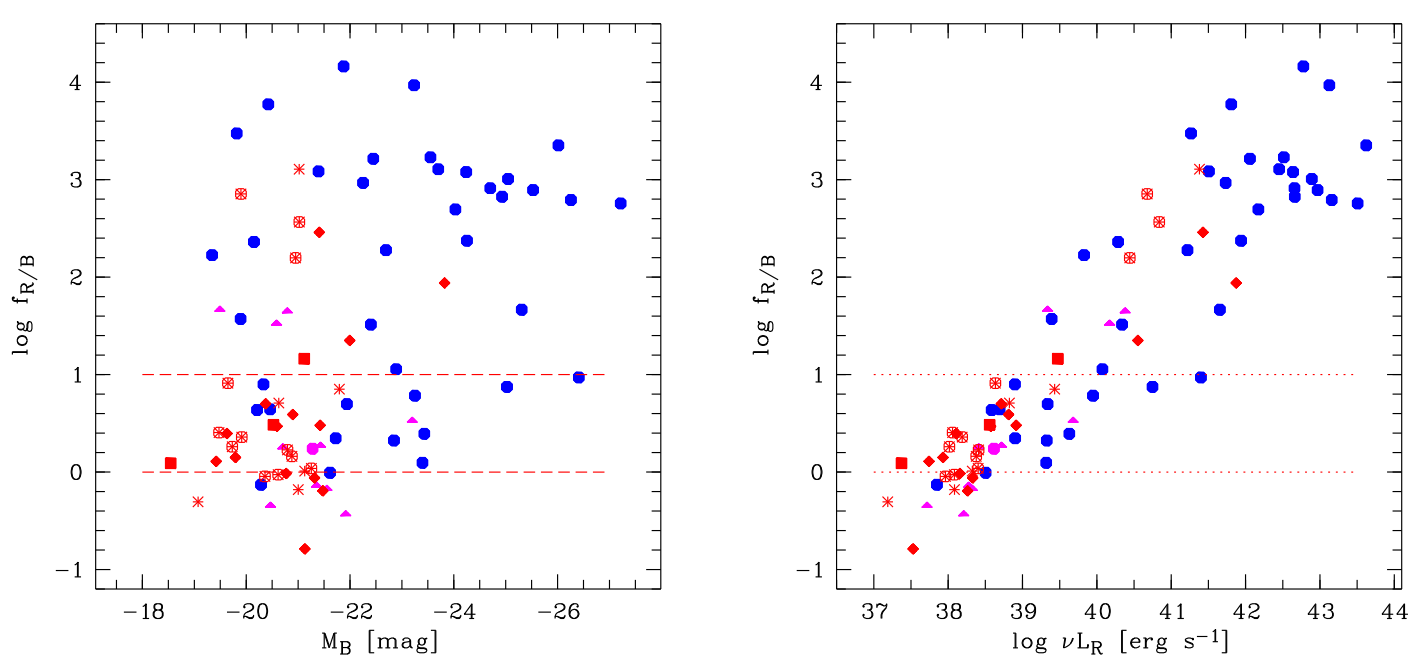

Abbildung 5.14: Das Flussverhältnis $\mathrm{f}_{\mathrm{R} / \mathrm{B}}$ als Funktion der optischen Absoluthelligkeit $\mathrm{M}_{\mathrm{B}}$ (links) bzw. der Radioleuchtkraft $v \mathrm{~L}_{\mathrm{R}}$ (rechts). Dieses Flussverhältnis dient nach Kellermann et al. (1989) zur Unterscheidung von radiolauten (RL) und radioleisen (RQ) Quellen, die entsprechenden Grenzen (RL: $\log f_{R / B}>1$ und RQ: $\left.\log f_{R / B}<0\right)$ sind als gestrichelte Linien eingezeichnet.

Tabelle 5.5: Samplegröße N, absolute und relative Häufigkeiten von radioleisen (RQ), radiolauten (RL) und intermediären (RI) Quellen sowie mittleres $\log f_{R / B}$ für die verschiedenen Untergruppen des Samples. Zum Vergleich sind außerdem die entsprechenden Werte für die 113 BQS-Quellen von Kellermann et al. (1989) mit angegeben.

\begin{tabular}{lcrccc}
\hline \hline & $\mathrm{N}$ & \multicolumn{1}{c}{$\mathrm{RQ}$} & $\mathrm{RI}$ & $\mathrm{RL}$ & $<\log \mathrm{f}_{\mathrm{R} / \mathrm{B}}>$ \\
\hline BQS & 113 & $79 / 70 \%$ & $12 / 11 \%$ & $22 / 19 \%$ & \\
VBLG & 39 & $2 / 5 \%$ & $11 / 28 \%$ & $26 / 67 \%$ & $2.02 \pm 1.27$ \\
XAGN & 49 & $12 / 25 \%$ & $24 / 49 \%$ & $13 / 26 \%$ & $0.68 \pm 0.94$ \\
XAGN-1 & 29 & $6 / 21 \%$ & $16 / 55 \%$ & $7 / 24 \%$ & $0.66 \pm 0.92$ \\
BL Lac & 3 & & $1 / 33 \%$ & $2 / 67 \%$ & $1.54 \pm 0.96$ \\
Sy1 & 17 & $4 / 24 \%$ & $9 / 52 \%$ & $4 / 24 \%$ & $0.55 \pm 0.80$ \\
Sy1.5...1.9 & 12 & $2 / 17 \%$ & $7 / 58 \%$ & $3 / 25 \%$ & $0.83 \pm 1.07$ \\
Sy2 & 6 & $2 / 33 \%$ & $3 / 50 \%$ & $1 / 17 \%$ & $0.70 \pm 1.27$ \\
LLAGN & 10 & $4 / 30 \%$ & $3 / 10 \%$ & $3 / 30 \%$ & $0.49 \pm 0.84$ \\
LINER & 1 & & $1 / 100 \%$ & & 0.24 \\
\hline \hline
\end{tabular}




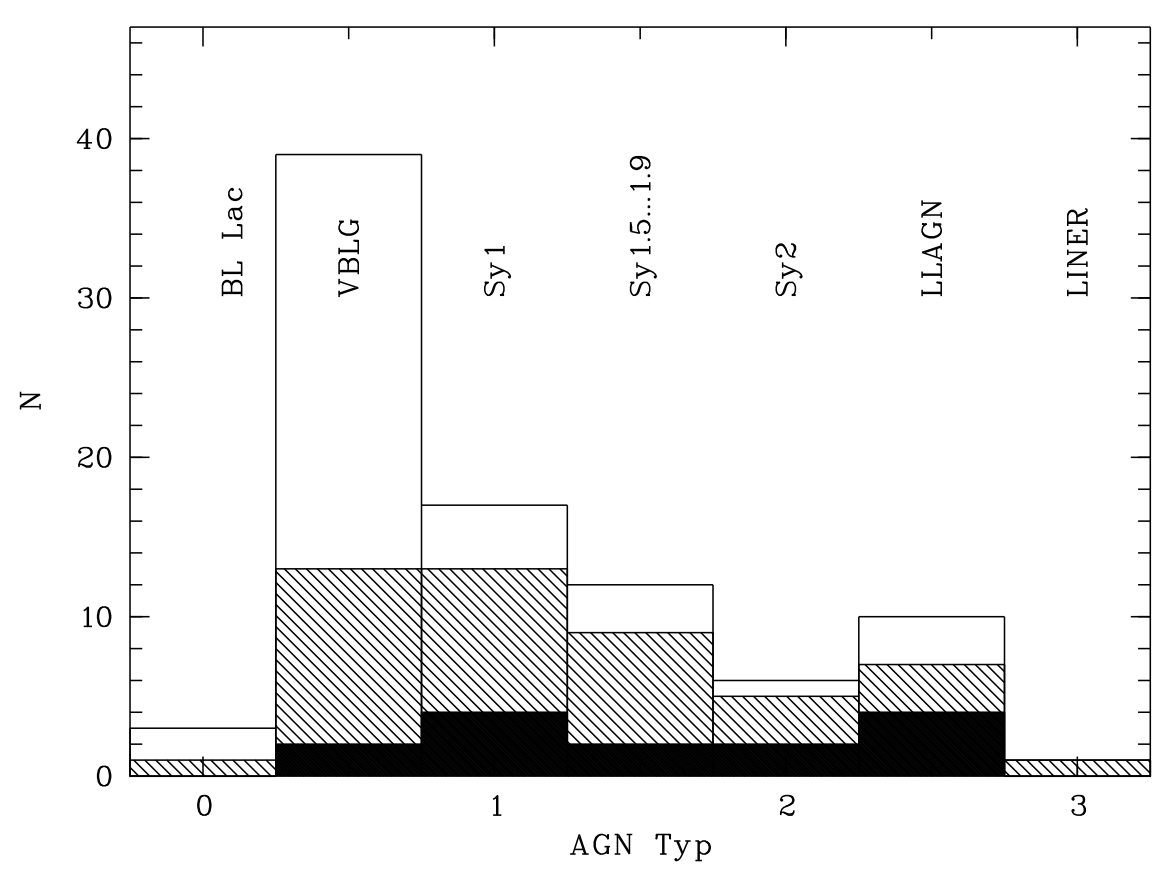

Abbildung 5.15: Häufigkeiten von radiolauten und radioleisen Quellen: schwarz eingezeichnet sind radioleise Quellen, schraffiert die Übergangstypen und nicht schraffiert radiolaute Quellen. Auffallend ist der hohe Anteil radiolauter Quellen unter den VBLG.

einzelnen AGN-Typen bestimmt. Die entsprechenden Werte sind in Tabelle 5.5 aufgelistet und in Abbildung 5.15 als Histogramm graphisch dargestellt. Während die VBLG zu 67\% radiolaut sind, sind es die XAGN nur zu 26\%. In dem in der Tabelle zum Vergleich mitangeführten, 113 Quellen umfassenden Palomar Bright Quasar Survey (BQS) Sample von Kellermann et al. (1989) ist der Anteil von radiolauten Galaxien mit 19\% noch etwas geringer.

Außerdem ist in Tabelle 5.5 auch das mittlere $\log \mathrm{f}_{\mathrm{R} / \mathrm{B}}$ für die einzelnen Untergruppen des Samples angegeben. Dieses ist bei den VBLG ebenfalls deutlich höher als bei den XAGN: die Differenz in $<\log \mathrm{f}_{\mathrm{R} / \mathrm{B}}>$ von 1.34 entspricht einem im Mittel zwanzigfach höherem $\mathrm{f}_{\mathrm{R} / \mathrm{B}}$ der VBLG im Vergleich zu den XAGN.

Bemerkenswert ist ferner, dass sich die XAGN insgesamt und die Typ-1 XAGN allein betrachtet in ihrer RL/RQ-Verteilung und insbesondere auch bezüglich ihres mittleren $\log \mathrm{f}_{\mathrm{R} / \mathrm{B}}$ praktisch überhaupt nicht unterscheiden.

Im Vergleich der beiden Diagramme der Abbildung 5.14 ist offensichtlich, dass $f_{R / B}$ in sehr viel stärkerem Maße von $v L_{R}$ als von $M_{B}$ abhängt. Dies wird von der Korrelationsanalyse eindeutig bestätigt: es gibt eine extrem starke Korrelation zwischen $\mathrm{f}_{\mathrm{R} / \mathrm{B}}$ und $v \mathrm{~L}_{\mathrm{R}}(\mathrm{r}=0.74, \mathrm{P}<0.001)$, ungleich stärker als die ebenfalls signifikante 


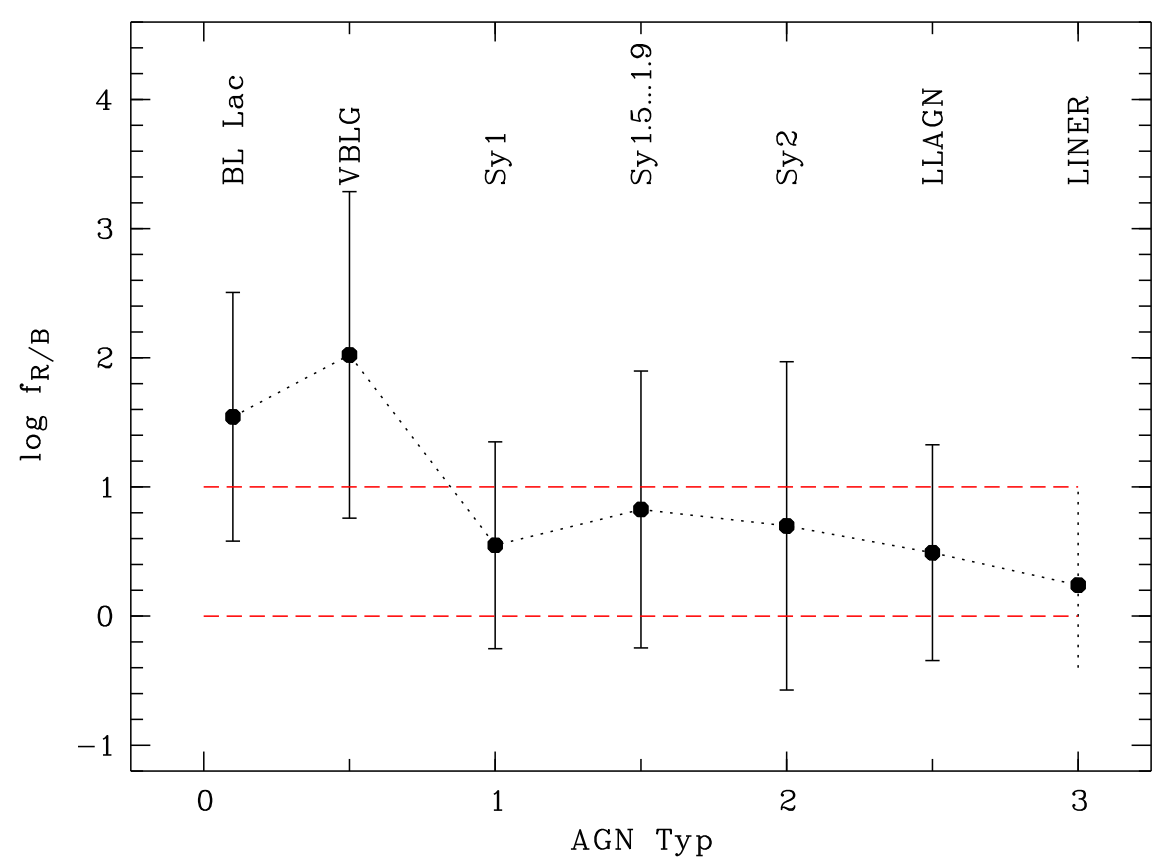

Abbildung 5.16: Das mittlere Flussverhältnis $f_{R / B}$ der einzelnen AGN-Typen. Auch hier sind zusätzlich die Grenzwerte für radiolaute bzw. radioleise Quellen eingezeichnet. Es ist ersichtlich, dass sich im Mittel die verschiedenen SeyfertTypen, LLAGN und LINER alle im intermediären Bereich befinden und sich bezüglich ihres mittleren $\mathrm{f}_{\mathrm{R} / \mathrm{B}}$ kaum unterscheiden, wohingegen die VBLG radiolaut sind.

Korrelation von $\mathrm{f}_{\mathrm{R} / \mathrm{B}}$ und $\mathrm{M}_{\mathrm{B}}(\mathrm{r}=-0.24, \mathrm{P}<0.001)$. Das bedeutet, das Flussverhältnis $\mathrm{f}_{\mathrm{R} / \mathrm{B}}$ wird von der Radioemission geprägt, und ist insofern konsistent mit dem Ergebnis von Kellermann et al. (1989), die bezüglich ihres BQS-Samples keine offensichtliche Abhängigkeit von $\mathrm{f}_{\mathrm{R} / \mathrm{B}}$ und $\mathrm{M}_{\mathrm{B}}$ fanden.

Auch für das Flussverhältnis $\mathrm{f}_{\mathrm{R} / \mathrm{B}}$ wurde eine Statistik für alle AGN-Typen einzeln berechnet. Die Ergebnisse sind in Abbildung 5.16 graphisch dargestellt. Man erkennt, dass im Mittel die VBLG das höchste $\mathrm{f}_{\mathrm{R} / \mathrm{B}}$ aufweisen. Ansonsten liegen nur noch die BL Lac Objekte im Bereich der radiolauten Quellen. Die Mittelwerte aller anderen Typen liegen im Rahmen der Genauigkeiten auf ähnlichem Niveau im Übergangsbereich zwischen radiolaut und radioleise. Damit wird das bereits angeführte bemerkenswerte Ergebnis untermauert, dass es diesbezüglich keinen nennenswerten Unterschied zwischen XAGN und Typ-1 XAGN gibt.

Die physikalischen Ursachen für die Existenz von radiolauten und radioleisen Quellen sind bis heute unklar und werden in der Literatur offen und kontrovers diskutiert. Frühere Hypothesen, z.B. dass radiolaute AGN nur in elliptischen und radioleise Quellen nur 

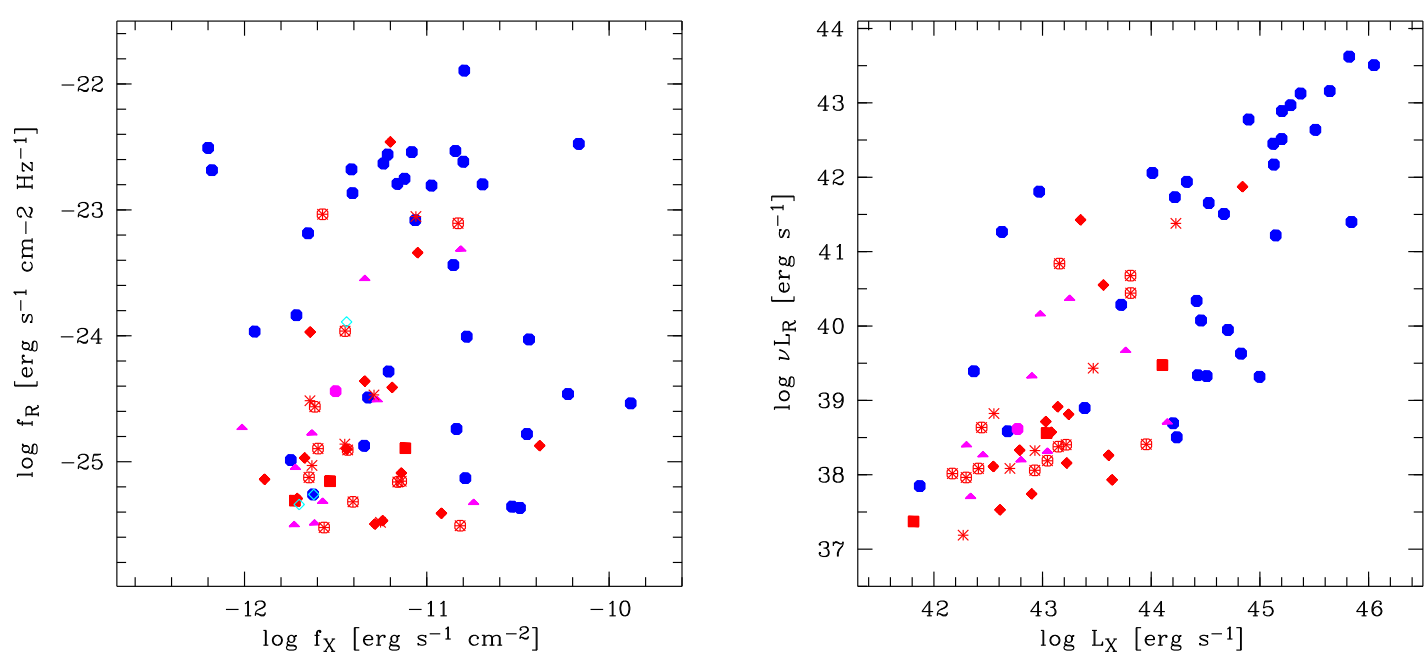

Abbildung 5.17: Radiofluss $\mathrm{f}_{R}$ gegen Röntgenfluss $\mathrm{f}_{\mathrm{X}}$ (links) bzw. Radioleuchtkraft $v L_{R}$ gegen die Röntgenleuchtkraft $L_{X}$ (rechts).

in Spiralgalaxien auftreten, wurden inzwischen wieder in Frage gestellt, relativiert oder widerlegt (z.B. McLure et al. 1999). Vielversprechende, aktuelle Arbeiten diskutieren die Masse des zentralen Schwarzen Lochs (Laor 2000, Ho \& Peng 2001), die Umgebung des AGN bzw. der Galaxie (McLure \& Dunlop 2001a,b, Percival et al. 2001) und die Akkretionsrate (Dopita 1997, 2000) als entscheidende Parameter für das Erscheinungsbild Aktiver Galaxien im Radiobereich.

\subsection{Verhältnis von Röntgen- zu Radiofluss $\mathbf{f}_{X / R}$}

In Abbildung 5.17 ist in der üblichen Weise der Radiofluss $\mathrm{f}_{R}$ gegen den Röntgenfluss $f_{X}$ bzw. die Radioleuchtkraft $v L_{R}$ gegen die Röntgenleuchtkraft $L_{X}$ dargestellt. Auch hier besteht zwischen den beiden Leuchtkräften eine sehr starke Korrelation ( $r=0.78$, $\mathrm{P}<0.001$ ), während für die Flüsse in dieser Abbildung keine Korrelation erkennbar ist und auch der Spearman-Test nur eine sehr geringe Korrelation $(r=0.20, P=0.07)$ ergibt.

Dies steht im Einklang mit Untersuchungen von radiolauten ROSAT-AGN, bei denen Reich et al. (2000) keine Korrelation zwischen Radio- und Röntgenfluss, Brinkmann et al. (1995) hingegen eine sehr starke Korrelation zwischen Radio- und Röntgenleuchtkraft fanden. 

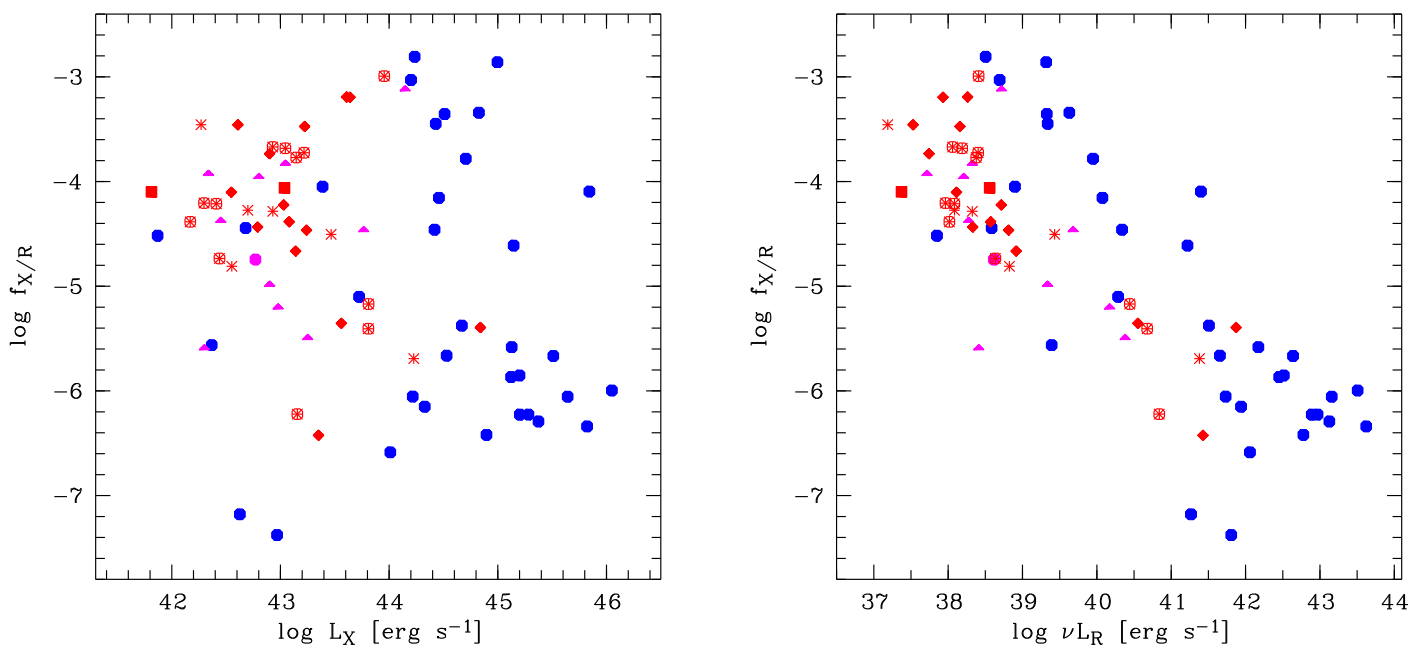

Abbildung 5.18: Das Flussverhältnis $f_{X / R}$ als Funktion der Röntgenleuchtkraft $\mathrm{L}_{\mathrm{X}}$ (links) bzw. der Radioleuchtkraft $\nu \mathrm{L}_{\mathrm{R}}$ (rechts). Die ungleich stärkere Korrelation von $\mathrm{f}_{\mathrm{X} / \mathrm{R}}$ mit der Radioleuchtkraft legt nahe, dass dieses Flussverhältnis wie auch $\mathrm{f}_{\mathrm{R} / \mathrm{B}}$ (s. Abbildung 5.14) - von der Radioemission dominiert wird.

Das Flussverhältnis von Röntgen- zu Radiofluss $f_{X / R}$ wird analog $z u f_{F I R / R}$ (Gleichung 5.5) als

$$
f_{X / R}=\frac{f_{X} / 4.84 \cdot 10^{17} \mathrm{~Hz}}{f_{R}}
$$

berechnet, wobei die Frequenz $4.84 \cdot 10^{17} \mathrm{~Hz}$ der Energie von $2 \mathrm{KeV}$ entspricht. Obschon einige Autoren das umgekehrte Verhältnis $\mathrm{f}_{\mathrm{R} / \mathrm{X}}$ verwenden (z.B. Yi \& Boughn 1998), wird hier obige Definition benutzt, um insbesondere den Vergleich mit den Arbeiten von Brinkmann et al. (1995) und Reich et al. (2000) zu erleichtern.

Abbildung 5.18 zeigt $f_{X / R}$ als Funktion der Röntgenleuchtkraft $L_{X}$ und der Radioleuchtkraft $v \mathrm{~L}_{\mathrm{R}}$. Es liegt eine schwache Antikorrelation von $\mathrm{f}_{\mathrm{X} / \mathrm{R}}$ mit $\mathrm{L}_{\mathrm{X}}(\mathrm{r}=-0.24$, $\mathrm{P}=0.03)$ und eine sehr starke mit $v \mathrm{~L}_{\mathrm{R}}(\mathrm{r}=-0.75, \mathrm{P}<0.001)$ vor. Dahingehend ähnelt das Verhältnis $f_{X / R}$ dem Verhältnis $f_{R / B}$, das ebenfalls eine sehr starke Korrelation mit $v L_{R}$ zeigt (s. Abschnitt 5.5) - beide Flussverhältnisse werden offenbar deutlich von der Radioemission dominiert.

Zur näheren Untersuchung des Zusammenhangs zwischen den Verhältnissen $f_{X / R}$ und $\mathrm{f}_{\mathrm{R} / \mathrm{B}}$ sind diese Größen in Abbildung 5.19 gegeneinander aufgetragen. Es zeigt sich wiederum eine äußerst starke Antikorrelation dieser beiden Flussverhältnisse ( $\mathrm{r}=-0.80$, $\mathrm{P}<0.001$ ). Ein entsprechendes Resultat erhielten bereits Brinkmann et al. (1995), wobei sie für Quasare und BL Lac Objekte eine deutlich stärkere Korrelation als für 


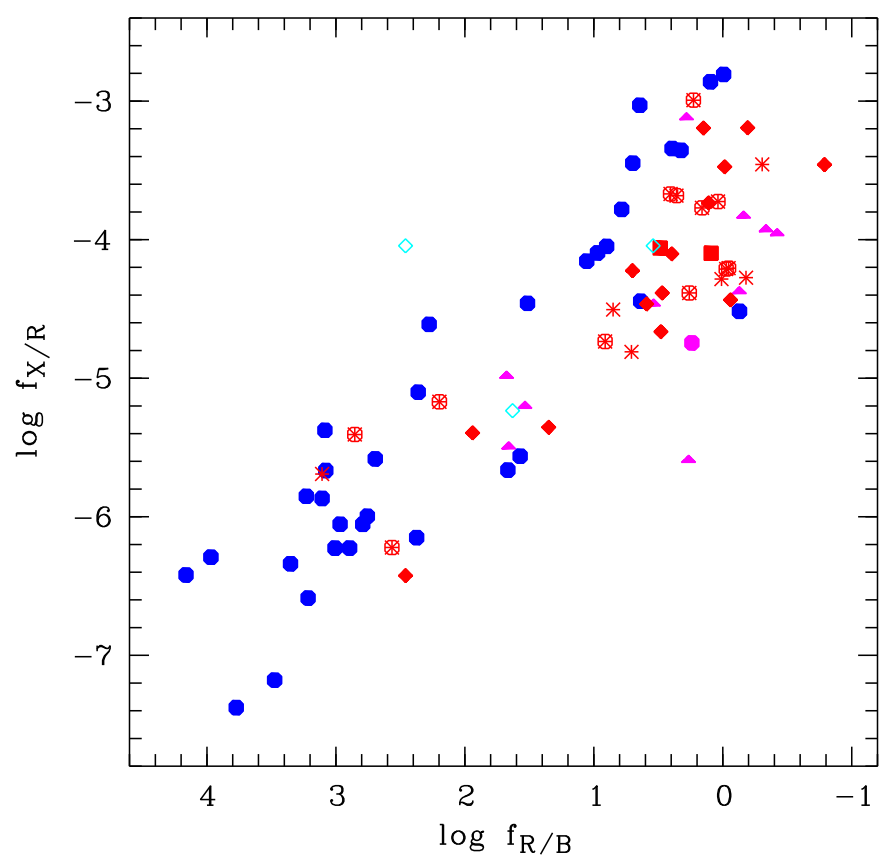

Abbildung 5.19: Die Flussverhältnisse $f_{X / R}$ und $f_{R / B}$. Zur besseren Vergleichbarkeit mit den entsprechenden Abbildungen von Brinkmann et al. (1995) und Reich et al. (2000) ist $f_{R / B}$ nach links zunehmend aufgetragen. Die von diesen Autoren gefundene Korrelation der beiden Größen ist auch hier offensichtlich.

Seyfert-Galaxien und nicht aktive Galaxien fanden. Reich et al. (2000) betrachten ebenfalls diese beiden Flussverhältnisse, quantifizieren aber nicht die offensichtlich weniger starke Korrelation für ihr Sample von radiolauten AGN. Die Korrelation impliziert eine Abhängigkeit der Form $L_{X} \sim L_{R}{ }^{\alpha} L_{B}{ }^{\beta}$, wie sie z.B. von Kembhavi et al. (1986) und Worrall et al. (1987) vorgeschlagen wurde.

Betrachtet man im hier vorliegende Sample die Untergruppen XAGN und VBLG getrennt, so lässt sich feststellen, dass die Korrelation für die VBLG stärker ( $\mathrm{r}=-0.90$, $\mathrm{P}<0.001)$ und für die XAGN entsprechend etwas geringer $(\mathrm{r}=-0.70, \mathrm{P}<0.001)$, in beiden Fällen aber eindeutig signifikant ausfällt. Die Dominanz der Radioemission ist bei den VBLG ausgeprägter als bei den XAGN.

Auf eine Darstellung der Abhängigkeit von $\mathrm{f}_{\mathrm{X} / \mathrm{R}}$ vom AGN-Typ wird verzichtet, da sich keine signifikanten Unterschiede zwischen den verschiedenen AGN-Typen ergaben. Die Mittelwerte aller AGN-Typen sind konsistent mit dem Mittelwert für das Gesamtsample von $<\log \mathrm{f}_{\mathrm{X} / \mathrm{R}}>=-4.7 \pm 1.1$. 
Tabelle 5.6: Ergebnisse der Korrelationsanalyse der sechs Flussverhältnisse mit der Rotverschiebung z für das Gesamtsample (AGN) wie auch für die beiden Untergruppen XAGN und VBLG. Angegeben ist jeweils die Größe der Stichprobe N, der Korrelationskoeffizient $r$ sowie die Wahrscheinlichkeit der Null-Hypothese P.

\begin{tabular}{l|ccc|ccc|crc}
\hline \hline & \multicolumn{3}{|c|}{ AGN } & \multicolumn{4}{|c|}{ XAGN } & \multicolumn{3}{|c}{ VBLG } \\
& $\mathrm{N}$ & $\mathrm{r}$ & $\mathrm{P}$ & $\mathrm{N}$ & $\mathrm{r}$ & $\mathrm{P}$ & $\mathrm{N}$ & \multicolumn{1}{c}{$\mathrm{r}$} & $\mathrm{P}$ \\
\hline $\mathrm{f}_{\mathrm{X} / \mathrm{B}}$ & 119 & 0.40 & $<0.001$ & 82 & 0.36 & $<0.001$ & 37 & 0.12 & 0.277 \\
$\mathrm{f}_{\mathrm{R} / \mathrm{B}}$ & 85 & 0.69 & $<0.001$ & 46 & 0.50 & $<0.001$ & 39 & 0.48 & 0.002 \\
$\mathrm{f}_{\mathrm{X} / \mathrm{R}}$ & 79 & -0.41 & $<0.001$ & 45 & -0.21 & 0.174 & 34 & -0.44 & 0.010 \\
$\mathrm{f}_{\mathrm{FIR} / \mathrm{R}}$ & 30 & -0.49 & 0.006 & 18 & -0.08 & 0.741 & 12 & -0.41 & 0.190 \\
$\mathrm{f}_{\mathrm{X} / \mathrm{FIR}}$ & 36 & 0.39 & 0.020 & 26 & 0.57 & 0.002 & 10 & 0.18 & 0.614 \\
$\mathrm{f}_{\mathrm{FIR} / \mathrm{B}}$ & 39 & 0.35 & 0.031 & 26 & 0.57 & 0.002 & 13 & 0.17 & 0.571 \\
\hline \hline
\end{tabular}

\subsection{Flussverhältnisse als Funktion der Rotverschiebung}

Wie bereits weiter oben kurz erwähnt ist einer der Vorteile bei der Betrachtung von Flussverhältnissen, dass sie prinzipiell entfernungsunabhängig sind. In diesem Abschnitt sollten die sechs Flussverhältnisse, die aus den Flüssen in den vier Frequenzbändern berechnet wurden, als Funktion der Rotverschiebung $z$ betrachtet werden.

Korrelationen zwischen Flussverhältnissen und der Rotverschiebung können Indikationen für eine kosmologische Entwicklung der Objekte sein. Sie können durch das bereits beschriebene (s. Abschnitt 5.1) Zusammenwirken von Beobachtungsmöglichkeiten und Leuchtkraftfunktion der Quellen hervorgerufen werden, insbesondere hätte eine unterschiedliche Entwicklung der Leuchtkraftfunktionen in verschiedenen Frequenzbändern (spektrale Entwicklung) eine Abhängigkeit des entsprechenden Flussverhältnisses von der Rotverschiebung zur Folge. Eine Übersicht über den derzeitigen Kenntnisstand zur Evolution von AGN gibt Osmer (2003). Hier soll vor allem auch untersucht werden, ob sich die Untergruppen XAGN und VBLG diesbezüglich gleich oder unterschiedlich verhalten.

Abbildung 5.20 zeigt alle sechs Flussverhältnisse als Funktion der Rotverschiebung. Es ist offensichtlich, dass die Stärke der Korrelationen deutlich unterschiedlich ist. Bemerkenswert ist, dass die Untergruppen XAGN und VBLG manchmal sehr verschiedene Bereiche der Diagramme bevölkern, manchmal aber auch kaum trennbar sind. Dabei ist zu bedenken, dass die unterschiedliche Größe der einzelnen Datensätze vor allem den rein visuellen Vergleich erschwert.

Die Ergebnisse der quantitativen Korrelationsanalyse sind in Tabelle 5.6 aufgelistet. 

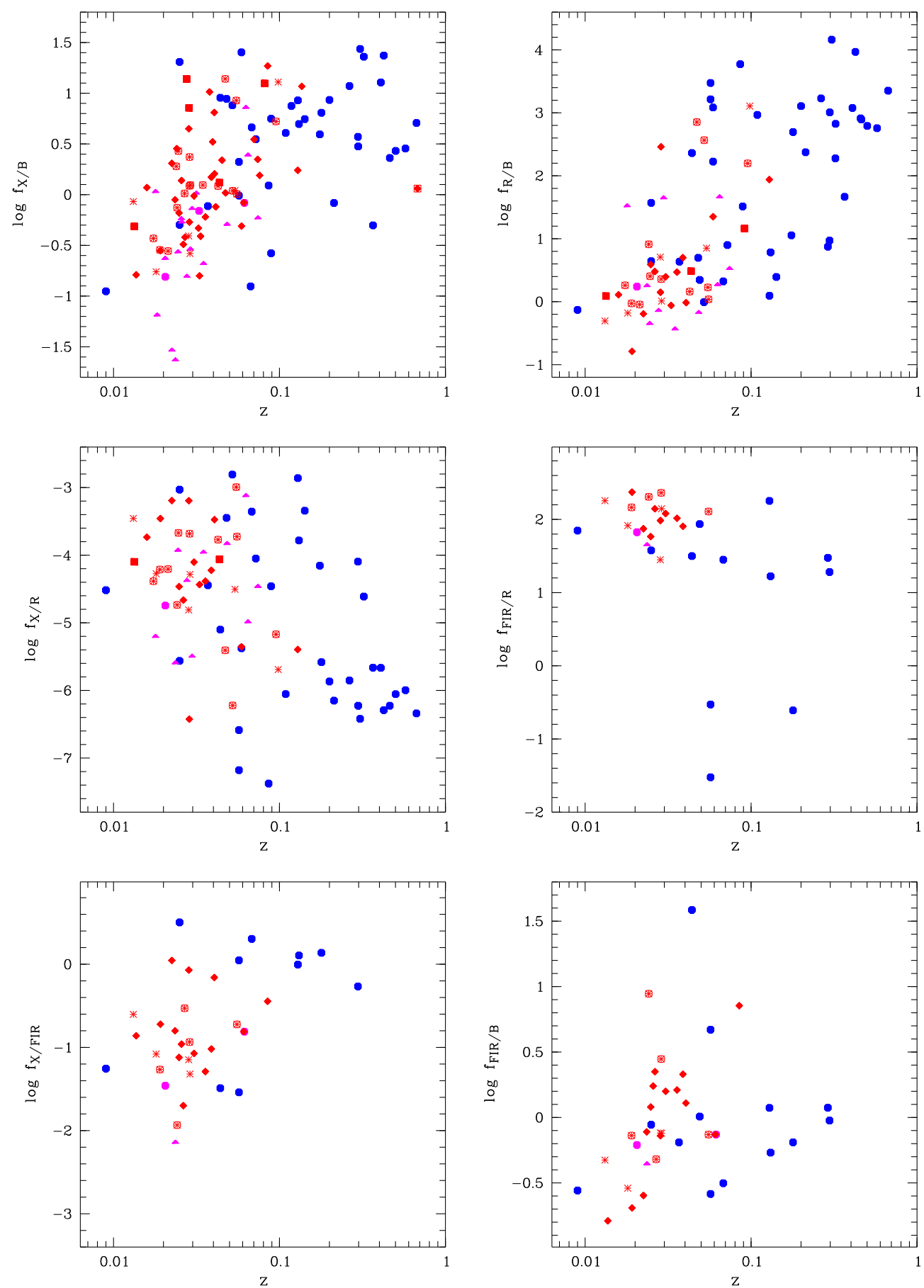

Abbildung 5.20: Die sechs Flussverhältnisse als Funktion der Rotverschiebung z. Die entsprechenden Ergebnisse der Korrelationsanalyse dieser Funktionen sind in Tabelle 5.6 aufgelistet. 
Dabei wurden alle Berechnungen sowohl für das Gesamtsample (AGN) als auch für die beiden Untergruppen XAGN und VBLG durchgeführt. Im Gesamtsample zeigt sich für alle sechs Flussverhältnisse eine signifikante Korrelation mit der Rotverschiebung. Allein die beiden schwächsten Korrelationen von $\mathrm{f}_{\mathrm{X} / \mathrm{FIR}}$ bzw. $\mathrm{f}_{\mathrm{FIR} / \mathrm{B}}$ mit $\mathrm{z}$ liegen mit $r<0.4$ in einem Bereich, in dem die Signifikanz der Korrelation nicht ganz sicher ist.

Betrachtet man hingegen die Untergruppen XAGN und VBLG einzeln, zeigt sich ein völlig anderes Bild: einige Flussverhältnisse sind für die jeweilige Untergruppe nicht mit z korreliert, wobei dies für die beiden Untergruppen jeweils deutlich unterschiedlich ist. Für die XAGN zeigen $f_{X / R}$ und $f_{F I R / R}$ keine Korrelation mehr mit $z$, für die VBLG sind sogar nur noch $f_{R / B}$ und $f_{X / R}$ mit $z$ korreliert.

Dass die Korrelationskoeffizienten bei der Betrachtung von Untergruppen kleiner werden, kann teilweise auch dadurch bedingt sein, dass diese Untergruppen naturgemäß kleiner als das Gesamtsample sind. Die soeben beschriebenen Unterschiede zwischen Gesamtsample und Untergruppen sind allerdings auch an der Wahrscheinlichkeit der Null-Hypothese P abzulesen, bei der die jeweilige Größe der entsprechenden Stichprobe berücksichtigt wird.

Dass hingegen in einigen Fällen (z.B. $f_{\mathrm{FIR} / \mathrm{B}}$ für XAGN - s. Tabelle 5.6) die Untergruppe deutlich stärker korreliert als das AGN Gesamtsample und dass im direkten Vergleich von XAGN und VBLG völlig unterschiedliche Korrelationen festzustellen sind, läßt sich keinesfalls mit statistischen Effekten der Stichprobengröße erklären. Es ist indessen ein starkes Indiz für intrinsische Unterschiede zwischen den beiden Untergruppen, die sich hier in einem deutlich unterschiedlichen Korrelationsverhalten hinsichtlich der Rotverschiebung niederschlagen.

Eine vergleichbare Untersuchung machten Brinkmann et al. (1995) für ein Sample von AGN, die sowohl im ROSAT-Röntgenkatalog als auch im 87GB-Radiokatalog enthalten sind. Sie fanden, dass $f_{X / B}$ unabhängig von der Rotverschiebung zu sein scheint, während $f_{R / B}$ und $f_{X / R}$ mit $z$ korrelieren. Im Vergleich mit obigen Resultaten unseres Samples ist dies bestens koinzident mit den VBLG, nicht jedoch mit den XAGN.

\subsection{Diskussion der Korrelationen}

Nachdem in den vorherigen Abschnitten vor allem auch die Besonderheiten und Auffälligkeiten einzelner Messgrößen und ihrer Verhältnisse diskutiert wurden, sollen nun die Ergebnisse noch einmal in Überblick betrachtet werden. Dazu eignen sich als die am besten untereinander vergleichbaren Ergebnisse diejenigen der Korrelationsanalyse. 
Tabelle 5.7: Ergebnisse der Korrelationsanalyse der vier Flüsse (obere Hälfte der Tabelle) sowie der vier Leuchtkräfte (untere Hälfte). Die Korrelationsanalyse bezog sich immer auf das Gesamtsample; eine Ausnahme bildet eine zusätzliche Analyse von $\mathrm{f}_{\text {FIR }}$ und $\mathrm{f}_{R}$, die sich auf das im Text definierte Sample AGN* bezog und in der letzten Tabellenspalte entsprechend gekennzeichnet ist.

\begin{tabular}{lrrcl}
\hline \hline Korrelation & \multicolumn{1}{c}{$\mathrm{N}$} & \multicolumn{1}{c}{$\mathrm{r}$} & $\mathrm{P}$ & Bemerkungen \\
\hline $\mathrm{m}_{\mathrm{B}} \times \mathrm{f}_{\mathrm{X}}$ & 123 & -0.02 & 0.790 & \\
$\mathrm{f}_{\mathrm{FIR}} \times \mathrm{f}_{R}$ & 30 & 0.27 & 0.152 & \\
$\mathrm{f}_{\mathrm{FIR}} \times \mathrm{f}_{R}$ & 27 & 0.61 & 0.001 & AGN* \\
$\mathrm{f}_{\mathrm{FIR}} \times \mathrm{f}_{\mathrm{X}}$ & 36 & -0.14 & 0.419 & \\
$\mathrm{~m}_{\mathrm{B}} \times \mathrm{f}_{\mathrm{FIR}}$ & 39 & -0.43 & 0.006 & \\
$\mathrm{~m}_{\mathrm{B}} \times \mathrm{f}_{R}$ & 88 & 0.24 & 0.027 & \\
$\mathrm{f}_{\mathrm{X}} \times \mathrm{f}_{R}$ & 83 & 0.20 & 0.071 & \\
$\mathrm{M}_{\mathrm{B}} \times \mathrm{L}_{\mathrm{X}}$ & 120 & -0.53 & $<0.001$ & \\
$\mathrm{~L}_{\mathrm{FIR}} \times \mathrm{vL}_{\mathrm{R}}$ & 30 & 0.85 & $<0.001$ & \\
$\mathrm{~L}_{\mathrm{FIR}} \times \mathrm{L}_{\mathrm{X}}$ & 36 & 0.68 & $<0.001$ & \\
$\mathrm{M}_{\mathrm{B}} \times \mathrm{L}_{\mathrm{FIR}}$ & 39 & -0.48 & 0.002 & \\
$\mathrm{M}_{\mathrm{B}} \times \mathrm{LL}_{\mathrm{R}}$ & 85 & -0.68 & $<0.001$ & \\
$\mathrm{~L}_{\mathrm{X}} \times \mathrm{vL}_{\mathrm{R}}$ & 80 & 0.78 & $<0.001$ & \\
\hline \hline
\end{tabular}

\subsubsection{Flüsse und Leuchtkräfte}

Die Flüsse wie auch die Leuchtkräfte der vier Spektralbereiche wurden jeweils untereinander auf Korrelationen untersucht. Die Resultate der Korrelationsanalysen sind in Tabelle 5.7 zusammengefasst. Die entsprechenden Abbildungen wurden bereits in den vorherigen Abschnitten 5.1 bis 5.6 gezeigt.

Es ist ersichtlich, dass alle vier Leuchtkräfte untereinander eindeutig korreliert sind; die Ursachen hierfür wurden bereits diskutiert.

Bei den vier Flüssen ist das Bild uneinheitlicher: die FIR-Radio-Korrelation wird anhand der Korrelationsergebnisse nur evident, wenn man das um die drei stark abweichenden Quellen reduzierte Sample AGN* betrachtet. Ebenfalls korreliert sind $\mathrm{m}_{\mathrm{B}}$ und $\mathrm{f}_{\mathrm{FIR}}$ sowie $\mathrm{m}_{\mathrm{B}}$ und $\mathrm{f}_{R}$.

Es fällt auf, dass der Röntgenfluss $f_{X}$ mit keinem anderen Spektralbereich signifikant korreliert. Das ist umso bemerkenswerter, da der Röntgendatensatz der zweitgrößte ist und somit eine zu geringe Samplegröße keinesfalls die Ursache sein kann. 
Tabelle 5.8: Ergebnisse der Korrelationsanalyse der sechs Flussverhältnisse $\mathrm{f}_{\mathrm{A} / \mathrm{B}}$ mit den zwei korrespondierenden Leuchtkräften $\mathrm{L}_{\mathrm{A}}$ und $\mathrm{L}_{\mathrm{B}}$. Die jeweils an erster Stelle aufgeführte Leuchtkraft korreliert sehr stark mit dem entsprechenden Flussverhältnis.

\begin{tabular}{lrrc}
\hline \hline Korrelation & $\mathrm{N}$ & $\mathrm{r}$ & $\mathrm{P}$ \\
\hline $\mathrm{f}_{\mathrm{X} / \mathrm{B}} \times \mathrm{L}_{\mathrm{X}}$ & 119 & 0.53 & $<0.001$ \\
$\mathrm{f}_{\mathrm{X} / \mathrm{B}} \times \mathrm{M}_{\mathrm{B}}$ & 119 & -0.08 & 0.174 \\
$\mathrm{f}_{\mathrm{FIR} / \mathrm{R}} \times \mathrm{vL}_{\mathrm{R}}$ & 30 & -0.71 & $<0.001$ \\
$\mathrm{f}_{\mathrm{FIR} / \mathrm{R}} \times \mathrm{L}_{\mathrm{FIR}}$ & 30 & -0.34 & 0.069 \\
$\mathrm{f}_{\mathrm{X} / \mathrm{FIR}} \times \mathrm{L}_{\mathrm{X}}$ & 36 & 0.68 & $<0.001$ \\
$\mathrm{f}_{\mathrm{X} / \mathrm{FIR}} \times \mathrm{L}_{\mathrm{FIR}}$ & 36 & 0.07 & 0.705 \\
$\mathrm{f}_{\mathrm{FIR} / \mathrm{B}} \times \mathrm{L}_{\mathrm{FIR}}$ & 39 & 0.57 & $<0.001$ \\
$\mathrm{f}_{\mathrm{FIR} / \mathrm{B}} \times \mathrm{M}_{\mathrm{B}}$ & 39 & 0.33 & 0.039 \\
$\mathrm{f}_{\mathrm{R} / \mathrm{B}} \times \mathrm{vL}_{\mathrm{R}}$ & 85 & 0.74 & $<0.001$ \\
$\mathrm{f}_{\mathrm{R} / \mathrm{B}} \times \mathrm{M}_{\mathrm{B}}$ & 85 & -0.24 & 0.001 \\
$\mathrm{f}_{\mathrm{X} / \mathrm{R}} \times \mathrm{vL}_{\mathrm{R}}$ & 79 & -0.75 & $<0.001$ \\
$\mathrm{f}_{\mathrm{X} / \mathrm{R}} \times \mathrm{L}_{\mathrm{X}}$ & 79 & -0.24 & 0.030 \\
\hline \hline
\end{tabular}

Generell impliziert die Korrelation zweier Größen, dass es eine gemeinsame physikalische Ursache bzw. eine kausale Verkettung derselben gibt.

\subsubsection{Flussverhältnisse}

Um zu untersuchen, ob die Flussverhältnisse $\mathrm{f}_{\mathrm{A} / \mathrm{B}}$ von einer der in sie eingehenden Größen A bzw. B dominiert werden, wurden sie auf Korrelationen mit den entsprechenden Leuchtkräften $\mathrm{L}_{\mathrm{A}}$ und $\mathrm{L}_{\mathrm{B}}$ analysiert. Die Ergebnisse sind in Tabelle 5.8 zusammengestellt. Wiederum finden sich die dazugehörigen graphischen Darstellungen bereits in den vorangehenden Abschnitten 5.1 bis 5.6.

Es zeigt sich, dass jeweils eine von den zwei in Frage kommenden Leuchtkräften stark mit dem entsprechenden Flussverhältnis korreliert, d.h. diese ist die bestimmende für das jeweilige Verhältnis. In Tabelle 5.8 ist sie immer an erster Stelle aufgeführt. Ist die Radioleuchtkraft $\nu \mathrm{L}_{\mathrm{R}}$ beteiligt, so ist jeweils sie die dominierende Größe. 
Die drei Flussverhältnisse $f_{F I R / B}, f_{R / B}$ und $f_{X / R}$ korrelieren auch mit der jeweils zweiten eingehenden Leuchtkraft, allerdings signifikant schwächer. Bei den anderen drei Flussverhältnissen ist das eindeutig nicht der Fall.

\subsubsection{Folgerungen aus den Korrelationen}

Unter den sechs Flussverhältnissen ist $\mathrm{f}_{\mathrm{X} / \mathrm{B}}$ auch deshalb besonders wichtig und aussagekräftig, weil es für nahezu das gesamte Sample vorliegt. Die hier erzielten Ergebnisse liefern zusammen mit schon von Maccacaro et al. (1988) gefundenen Resultaten starke Evidenz, dass $\mathrm{f}_{\mathrm{X} / \mathrm{B}}$ ein zuverlässiger Parameter für das Maß an Aktivität einer Quelle ist. Damit kann die in dieser Arbeit in Abschnitt 3.3 eingeführte Typ-Sequenz von 0.1 (BL Lac) bis 3 (LINER) auch als Aktivitäts-Sequenz verstanden werden.

Das hier vorliegende Sample ist aufgrund seiner Struktur gut geeignet, die für normale Galaxien gefundene Ferninfrarot-Radio-Korrelation auch für die verschiedenen Arten Aktiver Galaxien zu untersuchen. Es zeigte sich, dass die XAGN trotz ihrer Kernaktivität bezüglich der FIR-Radio-Korrelation sehr gut mit nicht aktiven Galaxien übereinstimmen. Unter den VBLG befinden sich drei Quellen, die ein sehr viel kleineres Flussverhältnis $\mathrm{f}_{\mathrm{FIR} / \mathrm{R}}$ als alle anderen Objekte zeigen und daher offensichtlich durch besondere Radioemission (möglicherweise Jets) dominiert werden - offenbar ist $\mathrm{f}_{\mathrm{FIR} / \mathrm{R}}$ gut geeignet, solche Quellen zu identifizieren. Die übrigen VBLG zeigen zwar auch eine FIR-Radio-Korrelation, allerdings mit einem niedrigeren mittleren $\mathrm{f}_{\mathrm{FIR} / \mathrm{R}}$ und größerer Streuung als sowohl die XAGN wie auch die Galaxien des Vergleichsamples von Helou et al. (1985). Die physikalische Ursache für die Ferninfrarot-Radio-Korrelation ist darin zu sehen, dass beide Spektralbereiche durch Emission von Sternentstehung dominiert werden (s. Abschnitt 5.2).

Aus dem Flussverhältnis $\mathrm{f}_{\mathrm{X} / \mathrm{FIR}}$ konnte gefolgert werden, dass für die meisten der Objekte des hier vorliegenden Samples der Starburst-Beitrag zur Röntgenemission keine wesentliche Rolle spielt. Weiterhin wurde eine starke Korrelation von $f_{X / F I R}$ mit der Röntgenleuchtkraft gefunden. Beide Ergebnisse sind so zu interpretieren, dass die Röntgenemission unserer Quellen von einem Aktiven Kern dominiert wird, während die FIR-Leuchtkraft der unterliegenden Galaxie zuzuordnen ist.

Für das Flussverhältnis $f_{\text {FIR/B }}$ findet sich eine Korrelation mit der FIR-Leuchtkraft $L_{\text {FIR, }}$, wie sie ähnlich bereits von Lehnert \& Heckman (1995) beschrieben wurde und deren Ursache auf (junge) Sternentstehungsausbrüche zurückgeführt werden kann. Zusammen mit der FIR-Radio-Korrelation bedeutet dieses Ergebnis, dass nicht nur FIR- und Radio-Emission, sondern in der Regel auch die optische B-Helligkeit der Quellen von Sternentstehung dominiert wird. Darüber hinaus ist jedoch bemerkenswert, dass diese Korrelation für die Untergruppen XAGN und $\mathrm{VBLG}^{+}$deutlich stärker ist als für das AGN Gesamtsample und dass sich für beiden Untergruppen signifikant unterschiedliche 
Regressionsgeraden errechnen. Ursache dafür könnte sein, dass nicht allein ein überall gleicher Starburst-Mechanismus in unterschiedlicher Stärke wirkt, sondern die Objekte der beiden Untergruppen zusätzlich intrinsische Unterschiede aufweisen. Es könnte allerdings auch ein anderer Parameter als der AGN-Typ für die auffällige Zweiteilung

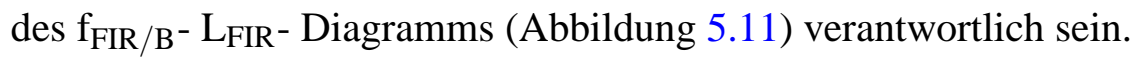

Zwischen radiolauten und radioleisen Quellen konnte anhand des Flussverhältnisses $\mathrm{f}_{\mathrm{R} / \mathrm{B}}$ unterschieden werden. Es zeigte sich, dass die VBLG im Mittel ein deutlich höheres $\mathrm{f}_{\mathrm{R} / \mathrm{B}}$ aufweisen. Während sie zu $67 \%$ radiolaut sind, sind es die XAGN nur zu $26 \%$. Dieses ist einer der wesentlichen Unterschiede der beiden Untergruppen; er ist indirekt durch das Selektionsverfahren bedingt. Die XAGN repräsentieren dabei „normale AGN“", was sich auch durch den Vergleich mit dem BQS-Sample von Kellermann et al. (1989) zeigt.

Das Flussverhältnis $f_{X / R}$ ähnelt dem Verhältnis $f_{R / B}$ in Bezug auf eine sehr starke Korrelation mit der Radioleuchtkraft $v \mathrm{~L}_{\mathrm{R}}$ - beide Flussverhältnisse werden offenbar deutlich von der Radioemission dominiert. Dementsprechend korrelieren auch die beiden Flussverhältnisse untereinander äußerst stark. Eine signifikante Abhängigkeit von AGN-Typ konnte für $\mathrm{f}_{\mathrm{X} / \mathrm{R}}$ nicht festgestellt werden.

Zusammenfassend betrachtet sprechen alle in diesem Abschnitt erzielten Resultate dafür, dass für die große Mehrheit der Quellen dieses Samples die Spektralbereiche R, FIR und B von Sternentstehung dominiert werden. Die Röntgenemission hingegen erfährt keinen nennenswerten Beitrag durch Sternentstehung und muss daher allein dem Aktiven Kern zugeschrieben werden.

Da die Ferninfrarot-Radio-Korrelation eine sehr enge Korrelation ist, konnten leicht drei in den entsprechenden Graphen sofort auffallende Quellen identifiziert werden, deren $\mathrm{f}_{\mathrm{FIR} / \mathrm{R}}$ um sieben bis zehn Standardabweichungen $\sigma$ unter dem Mittelwert der restlichen Quellen liegt und die somit eine sehr starke, zusätzliche Radiokomponente zeigen.

Sowohl bezüglich der FIR-R-Korrelation als auch der $\mathrm{f}_{\mathrm{FIR} / \mathrm{B}}-\mathrm{L}_{\mathrm{FIR}}-$ Korrelation zeigen sich signifikante Unterschiede zwischen den Untergruppen XAGN und VBLG (bzw. VBLG*/VBLG $^{+}$), die darauf hindeuten, dass es intrinsische physikalische Unterschiede zwischen den XAGN (bzw. ,,normalen“ AGN) und den VBLG geben kann. 


\section{Kapitel 6}

\section{Optische Spektroskopie der Broad-Line Region}

In diesem Kapitel werden wichtige Parameter der Broad-Line Region, die Breite der erlaubten Emissionslinien (FWHM) und das Balmerdekrement, mit in die Betrachtung des Samples einbezogen. Damit beschränkt es sich naturgemäß auf die Typ-1 AGN (inklusive der Übergangstypen bis Seyfert-1.9), da nur diese eine Broad-Line Region zeigen. Zur Verdeutlichung werden dieser Teil des Gesamtsamples im Folgenden auch mit AGN-1 und entsprechend die Typ-1 Objekte der Untergruppe XAGN auch mit XAGN-1 bezeichnet.

Wie in Abschnitt 7.3.3 noch genauer diskutiert werden wird, könnte die Breite der erlaubten Emissionslinien ein äußerst wichtiger Parameter sein. Bei einer scheibenförmigen Geometrie in der Broad-Line Region könnte er sogar ein direktes Maß des Blickwinkels auf diese Scheibe darstellen.

\subsection{Emissionslinienbreiten}

Als Linienbreite wurde primär die Halbwertsbreite FWHM (,full width at half maximum“) der Balmerlinien $\mathrm{H} \alpha$ und $\mathrm{H} \beta$ gemessen. In einigen Fällen konnte nur eine der Linien (meist $\mathrm{H} \alpha$ ) gemessen werden. In der Mehrzahl der Fälle wurde sowohl die Halbwertsbreite von $\mathrm{H} \alpha$ als auch von $\mathrm{H} \beta$ bestimmt und daraus der Mittelwert gebildet. Um eine einheitliche Messmethode auch für Spektren mit geringem Signal/Rausch-Verhältnis oder komplexen Linienprofilen zu verwenden, wurde die Linienbreite als FWHM eines Gauß-Fits an das Linienprofil bestimmt (s. Abschnitt 3.1).

Bei dem stark rotverschobenen QSO RX J023454.8-293425 liegen die Balmerlinien nicht mehr im beobachteten optischen Spektrum. Daher wurde stattdessen die Breite 


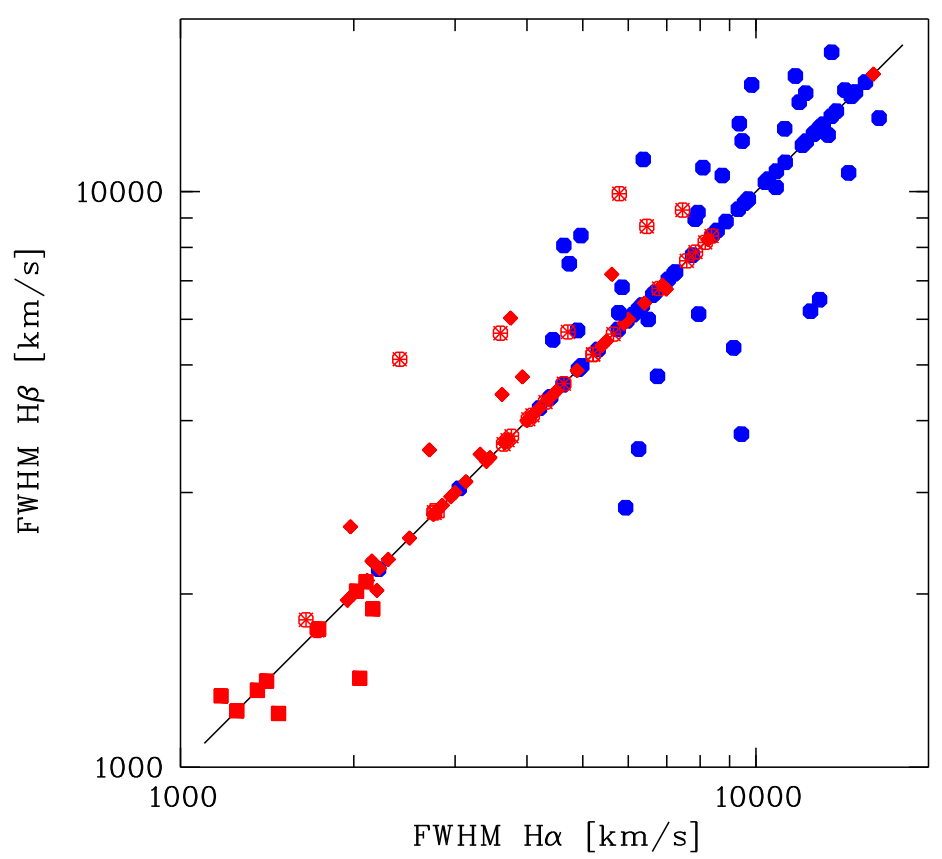

Abbildung 6.1: Für Objekte, bei denen die Halbwertsbreite sowohl von $\mathrm{H} \alpha$ als auch von $\mathrm{H} \beta$ gemessen werden konnte, sind beide Werte gegeneinander aufgetragen; zusätzlich ist der Mittelwert von $\mathrm{H} \alpha$ und $\mathrm{H} \beta$ auf der Diagonalen eingezeichnet. Für Objekte, bei denen nur eine der beiden Balmerlinien gemessen werden konnte, ist der entsprechende Wert ebenfalls auf der Diagonalen eingetragen.

der erlaubten Emissionslinie Mg II $\lambda 2798$ gemessen.

In Abbildung 6.1 ist für die Objekte mit breiten Balmerlinien die Halbwertsbreite von $\mathrm{H} \beta$ gegen die von $\mathrm{H} \alpha$ aufgetragen. Zusätzlich sind auf der Diagonalen die Mittelwerte aus beiden Halbwertsbreiten sowie für diejenigen Objekte, für die nur die Breite einer der Linien gemessen werden konnte, der entsprechende Wert aufgetragen.

Die Werte streuen um die Diagonale, für die FWHM H $\alpha=$ FWHM H $\beta$ gilt. Es gibt mehr Objekte, bei denen $\mathrm{H} \beta$ breiter ist als $\mathrm{H} \alpha$ (Messwerte oberhalb der Diagonalen) als umgekehrt. Dieses läßt sich im Rahmen von Modellen der Photoionisation durch eine zentrale Kontinuumsquelle verstehen (Rokaki et al. 1992, Collin-Souffrin \& Dumont 1989).

Der gleiche Effekt wird ebenfalls bei Variabilitätsuntersuchungen gefunden, bei denen die Ausdehnung bzw. der leuchtkraftgewichtete Zentrumsabstand $r$ der Entstehungsregion verschiedener Emissionslinien anhand von Laufzeitverzögerungen bestimmt wird (z.B. Bischoff, Kollatschny 1999, Kollatschny, Bischoff \& Dietrich 2000). Mit solchen Beobachtungen konnte auch bestätigt werden, dass die Gravitationskräfte auf das 


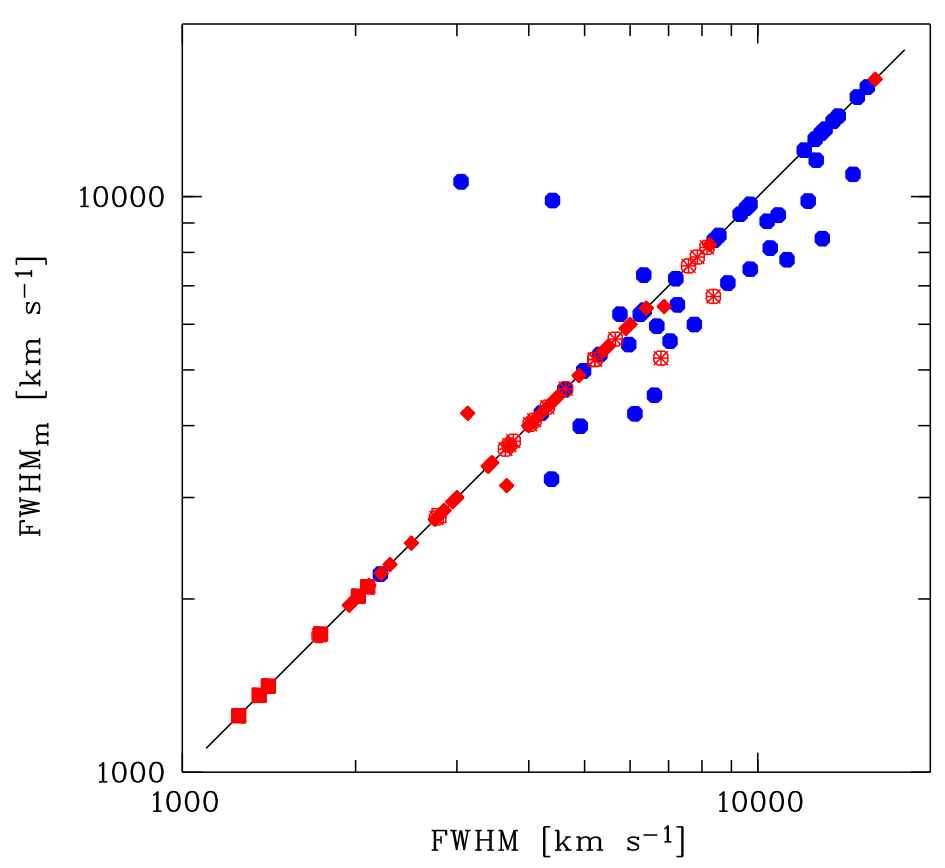

Abbildung 6.2: Zum Vergleich sind die wie im Text beschrieben auf unterschiedliche Arten bestimmten Halbwertsbreiten FWHM und $\mathrm{FWHM}_{m}$ gegeneinander aufgetragen. Die Abweichungen zwischen diesen sind verglichen mit den intrinsischen Unterschieden zwischen $\mathrm{H} \alpha$ und $\mathrm{H} \beta$ (s. Abbildung 6.1) gering.

linienemittierende Gas sehr viel stärker als der Strahlungsdruck sind und somit die BLR tatsächlich gravitativ gebunden ist und sich die Geschwindigkeiten $v$ einzelner Wolken im Gravitationspotenzial der Zentralmasse wie

$$
v \propto r^{-1 / 2}
$$

mit dem Abstand zum Zentrum $r$ verhalten (Peterson \& Wandel 1999, 2000) - dies ist ein wichtiges Ergebnis zum Verständnis der Linienbreiten.

Es ist allerdings auch zu berücksichtigen, dass die Messung der H $\alpha$ - bzw. H $\beta$-Linienbreiten jeweils anderen Schwierigkeiten unterliegen: während bei der H $\alpha$-Messung dies meist die Überlagerung durch die benachbarten [N II] 66548,6584 -Linien ist, ist es bei der H $\beta$-Messung i.d.R. die geringere Signalstärke.

Für 28 Galaxien wurde die breite $\mathrm{H} \alpha$ - oder $\mathrm{H} \beta$-Linie durch zwei Komponenten angepasst (s. Abschnitt 3.1). Ein Beispiel dafür ist die Galaxie PKS 2349-01, deren H $\alpha$ Linie mit den angepassten Linienkomponenten in Abbildung 3.1 dargestellt ist. In diesen Fällen wurde der Linienfluss aus der Summe beider Komponenten gebildet. Als 

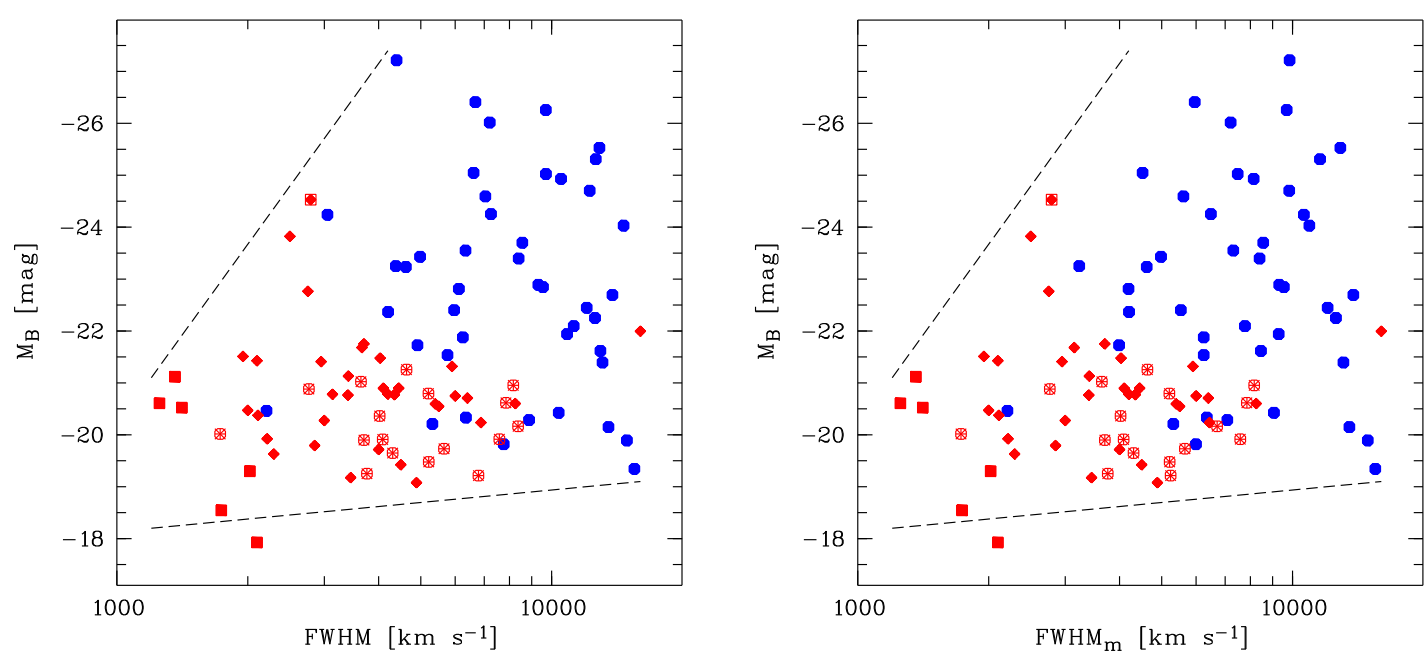

Abbildung 6.3: Um einen weiteren Vergleich von FWHM (links) und $\mathrm{FWHM}_{m}$ (rechts) zu führen, ist die optische Absoluthelligkeit $\mathrm{M}_{\mathrm{B}}$ gegen beide Größen aufgetragen. Bezüglich der generellen Trends, die im folgenden Abschnitt 6.2.1 genauer diskutiert werden, zeigen sich keine wesentlichen Unterschiede zwischen den beiden Abbildungen.

Linienbreite wurde die FWHM der breiteren Komponente benutzt, sofern diese mindestens ein Drittel des Gesamtflusses der breiten Linie enthielt. Gemäß Gleichung 6.1 entsteht die breitere Komponente näher am Zentrum des AGN, ist somit stärker vom diesem beeinflusst und damit von größerem Interesse.

Diese Vorgehensweise ist vergleichbar mit derjenigen in der Variabilitätsanalyse, bei z.B. die Masse des zentralen Schwarzen Lochs aus dem rms-Anteil des Linienprofils bestimmt wird; das rms-Profil stellt den zeitvariablen Linienanteil dar, ist i.d.R. breiter als das Gesamtprofil und entsteht dementsprechend zentrumsnäher (Kollatschny \& Bischoff 2002).

Zum Vergleich wurde aus der breiten (b) und der sehr breiten (v) Komponente ein flussgewichteter Mittelwert $\mathrm{FWHM}_{m}$ der Linienbreite gemäß

$$
F W H M_{m}=\frac{F W H M_{b} \cdot f_{b}+F W H M_{v} \cdot f_{v}}{f_{b}+f_{v}}
$$

berechnet. Dieser ist in Abbildung 6.2 gegen die zuvor bestimmte Halbwertsbreite FWHM aufgetragen. FWHM $m$ ist im Mittel etwas kleiner als FWHM, da in jedem Fall auch die weniger breite Linienkomponente eingeht. Die Abweichungen sind im Vergleich zu den intrinsischen Unterschieden zwischen $\mathrm{H} \alpha$ und $\mathrm{H} \beta$ (s. Abbildung 6.1) aber gering. 


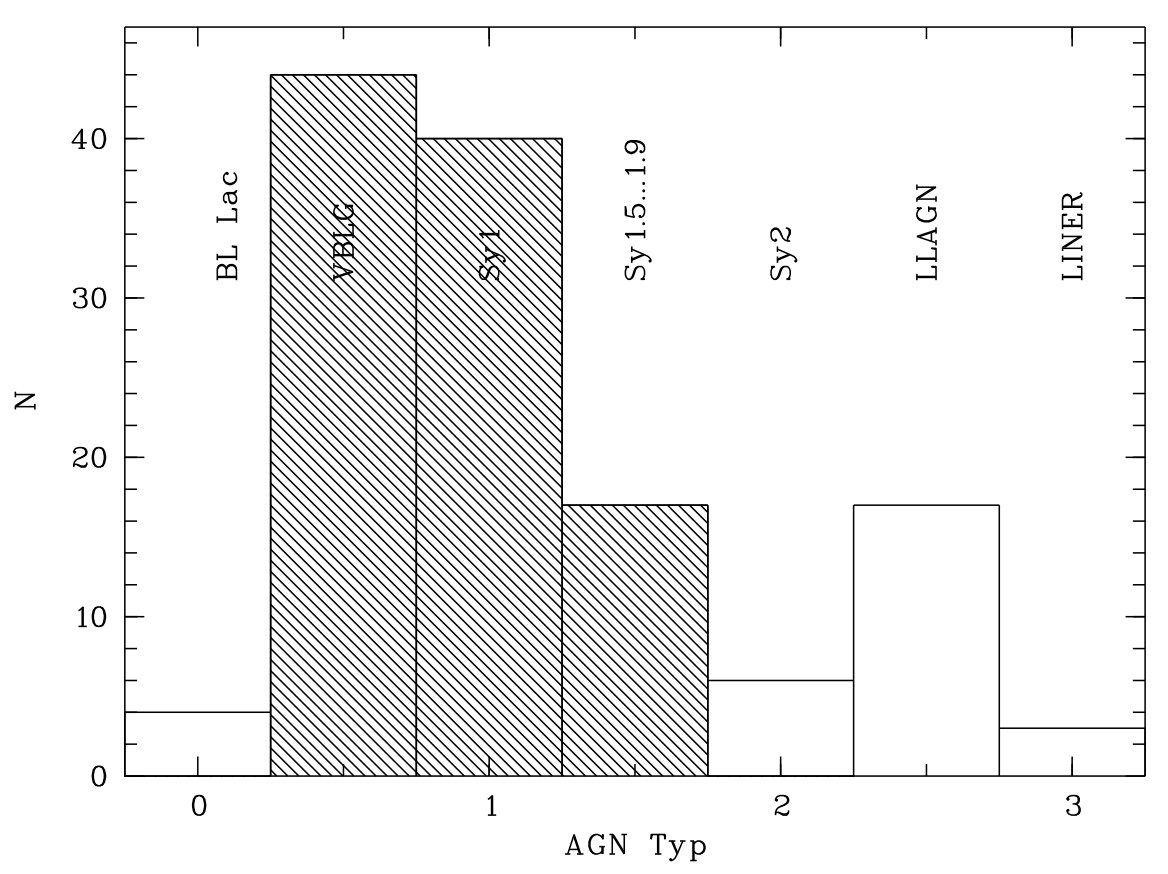

Abbildung 6.4: Anteil der AGN-Typen mit breiten Linienkomponenten. Schraffiert dargestellt sind diejenigen AGN, für die Halbwertsbreiten breiter Balmerlinienkomponenten gemessen werden konnten. Naturgemäß handelt es sich dabei um die Typ-1 AGN; diese Abbildung zeigt der Vollständigkeit halber, dass bei diesen ohne Ausnahme breite Balmerlinienkomponenten gemessen werden konnten.

Um in einem weiteren Vergleich die Unterschiede beurteilen zu können, wurde in Abbildung 6.3 die optische Absoluthelligkeit $\mathrm{M}_{\mathrm{B}}$ sowohl gegen FWHM als auch gegen $\mathrm{FWHM}_{m}$ aufgetragen. Es sind nur geringfügige Unterschiede sichtbar. In Bezug auf die generellen Trends, die im folgenden Abschnitt 6.2.1 genauer diskutiert werden, zeigen sich keine wesentlichen Abweichungen.

Eine Liste der Galaxien, bei denen breite Balmerlinien durch zwei Komponenten angepasst wurden, ist in Tabelle $8.3 \mathrm{im}$ Anhang zu finden. Es ist für $\mathrm{H} \alpha$ und $\mathrm{H} \beta$ jeweils die Halbwertsbreite FWHM der breiten (b) und der sehr breiten (v) Komponenten sowie der relative Flussanteil der sehr breiten Komponente $f_{v} / f_{\text {tot }}$ aufgelistet. Ferner ist die flussgewichtet gemittelte Halbwertsbreite $\mathrm{FWHM}_{m}$ und der Mittelwert der relativen Flussanteile der sehr breiten $\mathrm{H} \alpha$ - und $\mathrm{H} \beta$-Komponenten aufgeführt.

In Abbildung 6.4 ist der Vollständigkeit halber der Anteil der verschiedenen AGNTypen schraffiert dargestellt, für den Halbwertsbreiten von breiten Balmerlininenkomponenten gemessen werden konnten. Naturgemäß ist das nur bei den Typ-1 AGN (VBLG, Seyfert-1 und Übergangstypen Seyfert-1.5, -1.8, -1.9) der Fall, bei denen aber ausnahmslos breite Komponenten gemessen werden konnten. Nur diese Typen sind 


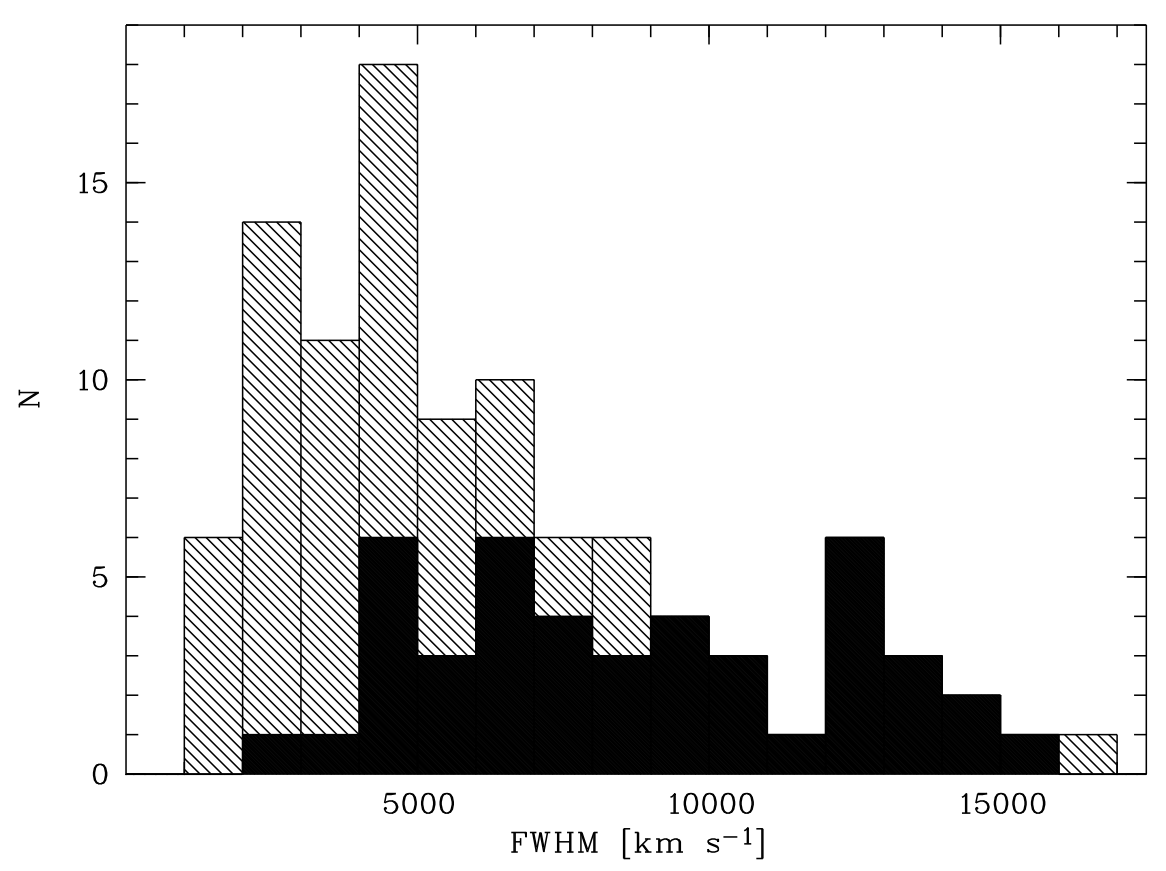

Abbildung 6.5: Häufigkeitsverteilung der für $\mathrm{H} \alpha$ bzw. $\mathrm{H} \beta$ gemessenen Linienhalbwertsbreiten. Schwarz dargestellt sind die VBLG, die bei großen Linienbreiten nahezu die gesamte Population stellen, die schraffierten Bereiche der Balken entsprechen den XAGN. Bemerkenswert ist der äußerst große Bereich von Halbwertsbreiten, den das Sample durchgehend überdeckt.

gemeint, wenn künftig von Linienbreiten die Rede ist.

Im Folgenden wird der Mittelwert der Halbwertsbreiten von $\mathrm{H} \alpha$ und $\mathrm{H} \beta$ verwendet, falls diese bei beiden Linien gemessen werden konnte, ansonsten natürlich die Halbwertsbreite der jeweils messbaren Linie.

Abbildung 6.5 zeigt die Häufigkeitsverteilung dieser Halbwertsbreiten. Das Gesamtsample überdeckt durchgehend den äußerst großen Bereich von $1000 \mathrm{~km} \mathrm{~s}^{-1}$ bis 16000 $\mathrm{km} \mathrm{s}^{-1}$. Es ist ersichtlich, dass die schwarz dargestellten VBLG nahezu die gesamte Population der Objekte mit sehr großen Halbwertsbreiten (FWHM $>9000 \mathrm{~km} \mathrm{~s}^{-1}$ ) stellen. Außerdem ist ihre Verteilung sehr viel breiter als die der XAGN (schraffierte Bereiche), die stark zwischen $2000 \mathrm{~km} \mathrm{~s}^{-1}$ und $5000 \mathrm{~km} \mathrm{~s}^{-1}$ konzentriert sind.

Als einzige Ausnahme fällt mit der größten Linienbreite aller Objekte ESO 416-G 002 auf. Nach dem optischen Spektrum hätte diese Galaxie auch als VBLG klassifiziert werden können. Sie entstammt jedoch dem röntgenselektiertem XAGN-Sample und wird daher hier als Seyfert-1 geführt. Die Halbwertsbreite ihrer recht schwachen breiten $\mathrm{H} \alpha$ Linienkomponente wurde zu $16000 \mathrm{~km} \mathrm{~s}^{-1}$ abgeschätzt. 
Tabelle 6.1: Statistik der Halbwertsbreiten der Balmerlinien $\mathrm{H} \alpha$ und $\mathrm{H} \beta$ für das Gesamtsample (AGN-1) sowie die beiden Untersamples XAGN-1 und VBLG. Die Mittelwerte wurden wie im Text beschreiben errechnet - diese werden für die weitere Analyse benutzt.

\begin{tabular}{lccc}
\hline \hline & \multicolumn{3}{c}{ FWHM / km s } \\
& $\mathrm{H} \alpha$ & $\mathrm{H} \beta$ & Mittelwert \\
\hline AGN-1 & $6000 \pm 3690$ & $7420 \pm 4240$ & $6220 \pm 3720$ \\
XAGN-1 & $4070 \pm 2390$ & $4490 \pm 2590$ & $4250 \pm 2450$ \\
VBLG & $8810 \pm 3440$ & $9170 \pm 4080$ & $8760 \pm 3550$ \\
\hline \hline
\end{tabular}

Es gibt zwei Samples so genannter „Double-peaked emission line AGN“ (DP-AGN) in der Literatur (s. Abschnitt 2.1), die hinsichtlich der Häufigkeitsverteilung der BalmerHalbwertsbreiten mit diesem Sample vergleichbar sind: beide Samples enthalten ebenfalls auch Objekte mit sehr großen Linienbreiten und die entsprechenden Abbildungen 10a) von Strateva et al. (2003) sowie 4a) von Eracleous \& Halpern (2003) ähneln der Abbildung 6.5.

Tabelle 6.1 listet die Mittelwerte der Linienhalbwertsbreiten für das Gesamtsample (AGN-1) sowie die beiden Untersamples XAGN-1 und VBLG auf. In den Spalten H $\alpha$ und $\mathrm{H} \beta$ sind jeweils die entsprechenden Mittelwerte und Standardabweichungen aller messbaren Linien aufgeführt. Für die Mittelwerte wurde zunächst gegebenenfalls ein Mittelwert aus $\mathrm{H} \alpha$ und $\mathrm{H} \beta$ gebildet (s.o.) und dieser wiederum über alle Objekte des entsprechenden Samples gemittelt. Da für $\mathrm{H} \alpha$ mehr Messungen als für $\mathrm{H} \beta$ vorliegen, liegt der resultierende Mittelwert näher am $\mathrm{H} \alpha$ - als am $\mathrm{H} \beta$-Wert. Dieser Mittelwert wird bei den folgenden Betrachtungen verwendet.

Das auffälligste Ergebnis dieser Statistik ist, dass die mittleren Halbwertsbreiten der VBLG mehr als doppelt so hoch wie die der XAGN sind und dass auch die Standardabweichungen deutlich höher sind; das spiegelt die für die Abbildung 6.5 beschriebene breitere Verteilung der VBLG bei höheren Halbwertsbreiten wider.

Weiterhin wird hier nochmals quantitativ belegt, dass im Mittel $\mathrm{H} \beta$ breiter als $\mathrm{H} \alpha$ ist. Dieser Effekt wurde bereits anhand der Abbildung 6.1 ersichtlich und seine physikalischen Ursachen wurden in diesem Zusammenhang diskutiert (s.o.).

Abschließend wird die Linienhalbwertsbreite als Funktion der Rotverschiebung betrachtet; dazu sind in Abbildung 6.6 beide Größen gegeneinander aufgetragen. Wie bereits gezeigt haben die VBLG gegenüber den XAGN im Mittel eine höhere Rotverschiebung und eine höhere Linienbreite. Daher befinden sie sich in dieser Abbildung überwiegend 

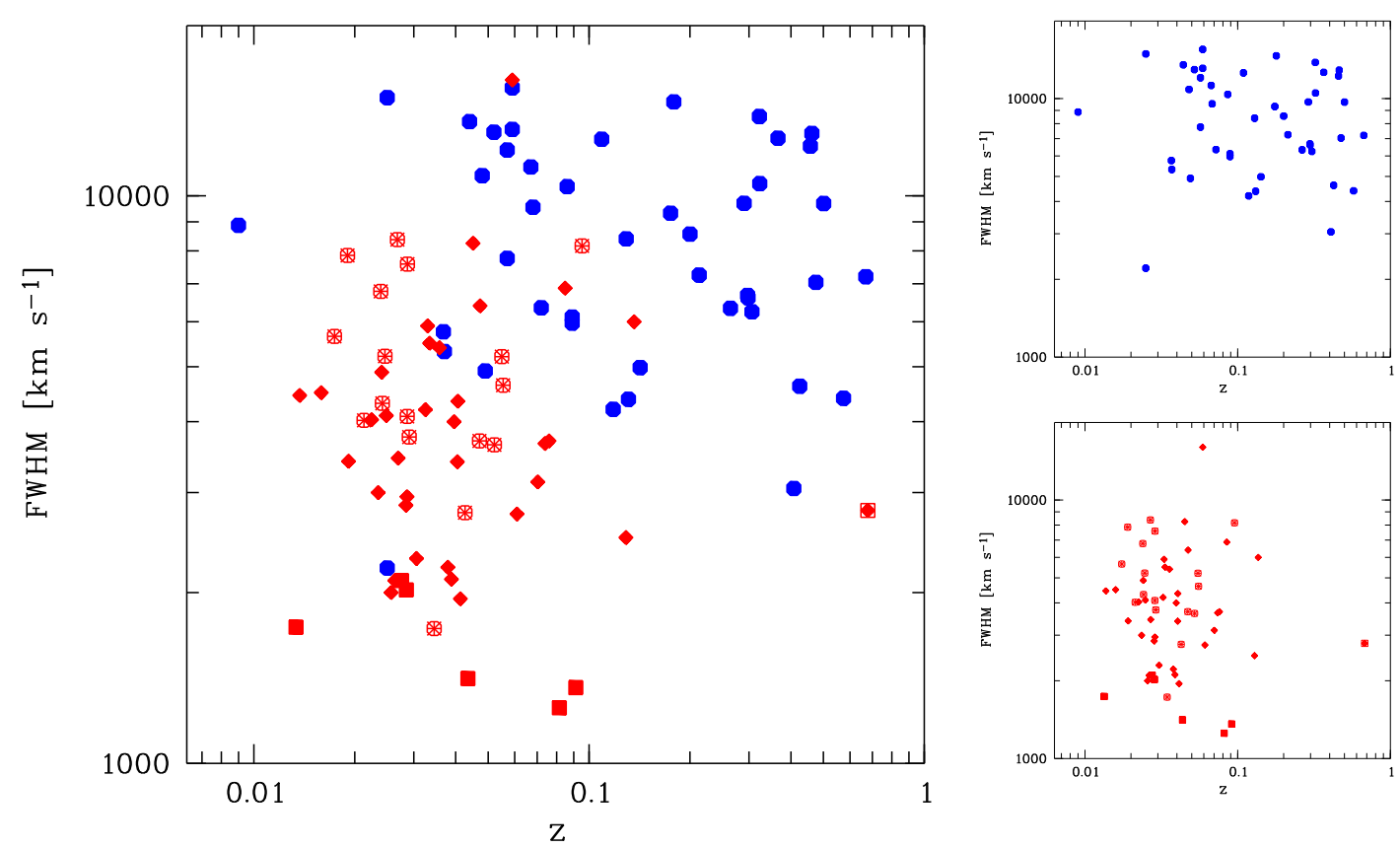

Abbildung 6.6: Linienbreiten als Funktion der Rotverschiebung. Die Schwerpunkte der beiden Untergruppen bezüglich Linienbreite und Rotverschiebung sind unterschiedlich, was insbesondere in der separaten Darstellung in den kleineren Diagrammen (rechts oben: VBLG; rechts unten: XAGN-1) deutlich wird. Innerhalb der Untergruppen zeigt sich jedoch keinerlei Zusammenhang zwischen beiden Größen.

im rechten oberen Teil des Diagramms, während die XAGN entsprechend eher im linken unteren Bereich liegen. Insgesamt betrachtet dominiert aber die Streuung über den nahezu vollständigen Bereich des Diagramms.

Die in Tabelle 6.2 aufgelisteten Ergebnisse der Korrelationsanalyse zeigen eindeutig, dass es zwar für das Gesamtsample eine Korrelation zwischen Halbwertsbreite und Rotverschiebung gibt, nicht jedoch innerhalb der beiden Untergruppen XAGN und VBLG. Das ist offensichtlich dadurch bedingt, dass die beiden Untergruppen aufgrund ihrer Selektion unterschiedliche Schwerpunkte bezüglich Linienbreite und Rotverschiebung haben und dadurch die beiden an sich nicht korrelierten Größen in Gesamtsample betrachtet wieder eine Korrelation zeigen, die zwar nicht stark $(r=0.24)$, wegen des großen Samples $(N=101)$ aber signifikant ist.

Zusammenfassend sei festgehalten, dass für alle Typ-1 AGN inklusive der Übergangstypen bis Seyfert-1.9 eine Linienbreite bestimmt werden konnte. Während die VBLG eine breite Verteilung mit einem sehr hohen Mittelwert von $8760 \mathrm{~km} \mathrm{~s}^{-1}$ zeigen, konzentrieren sich die XAGN stark zwischen $2000 \mathrm{~km} \mathrm{~s}^{-1}$ und $5000 \mathrm{~km} \mathrm{~s}^{-1}$. Zusammen- 
Tabelle 6.2: Ergebnisse der Korrelationsanalyse zwischen Linienhalbwertsbreite FWHM und Rotverschiebung z. Für das Gesamtsample (AGN-1) findet sich eine signifikante Korrelation, in den beiden Untergruppen XAGN-1 und VBLG für sich genommen sind die Größen hingegen nicht korreliert.

\begin{tabular}{lrrr}
\hline \hline & $\mathrm{N}$ & \multicolumn{1}{c}{$\mathrm{r}$} & \multicolumn{1}{c}{$\mathrm{P}$} \\
\hline AGN-1 & 101 & 0.24 & $<0.001$ \\
XAGN-1 & 57 & -0.05 & 0.563 \\
VBLG & 44 & -0.07 & 0.478 \\
\hline \hline
\end{tabular}

genommen weist das Sample eine durchgehende Verteilung bis hin zu außergewöhnlich hohen $16000 \mathrm{~km} \mathrm{~s}^{-1}$ auf.

\subsection{Korrelationen mit der Linienbreite}

Um mögliche Zusammenhänge der verschiedenen Messgrößen mit der Linienhalbwertsbreite genauer zu untersuchen, wurde der FWHM-Parameterraum in folgende vier Intervalle aufgeteilt:

$$
\begin{array}{lc}
\text { I: } & 1000-2500 \mathrm{~km} \mathrm{~s}^{-1} \\
\text { II: } & 2500-5000 \mathrm{~km} \mathrm{~s}^{-1} \\
\text { III: } & 5000-10000 \mathrm{~km} \mathrm{~s}^{-1} \\
\text { IV: } & >10000 \mathrm{~km} \mathrm{~s}^{-1}
\end{array}
$$

Dabei umfasst der Bereich I Typ-1 Objekte mit schmalen Linien, insbesondere auch die Narrow-Line Seyfert-1 Galaxien (NLS1), in den Bereich II fallen typische Typ-1 Galaxien, Bereich III umfasst Objekte mit sehr breiten und Bereich IV solche mit ultrabreiten Linien.

\subsubsection{Leuchtkräfte}

Für diese vier Halbwertsbreitenintervalle wurden jeweils separat die statistischen Parameter der verschiedenen Beobachtungsgrößen berechnet. Die entsprechenden Daten (Gruppengröße N, Mittelwert $<\mathrm{L}>$ und Standardabweichung $\sigma$ ) bezüglich der Leuchtkräfte der vier Frequenzbänder sind in Tabelle 6.3 aufgelistet und in den Graphen der 
Abbildung 6.7 eingezeichnet. Weiterhin sind in Tabelle 6.3 die statistischen Parameter für das Gesamtsample AGN-1 sowie für die beiden Untergruppen XAGN und VBLG aufgeführt.

Tabelle 6.3: Statistik der Leuchtkräfte in den vier Frequenzbändern B, X, FIR und $\mathrm{R}$ jeweils für das Gesamtsample AGN-1, die beiden Untergruppen XAGN-1 und VBLG sowie für die vier oben definierten Halbwertsbreitenintervalle I - IV.

\begin{tabular}{l|rcc|ccc}
\hline \hline & \multicolumn{3}{|c|}{$\begin{array}{c}\mathrm{M}_{\mathrm{B}}[\mathrm{mag}] \\
\mathrm{N}\end{array} \mathrm{M}_{\mathrm{B}}>$} & $\sigma$ & $\mathrm{N}$ & $\left.<\log \mathrm{L}_{\mathrm{X}}>\mathrm{erg} \mathrm{s}^{-1}\right]$ \\
\hline AGN-1 & 101 & -21.58 & 1.95 & 94 & 43.70 & 1.00 \\
XAGN-1 & 57 & -20.56 & 1.12 & 57 & 43.25 & 0.64 \\
VBLG & 44 & -22.90 & 2.01 & 37 & 44.40 & 1.06 \\
FWHM I & 14 & -20.13 & 1.02 & 14 & 43.28 & 0.65 \\
FWHM II & 35 & -21.38 & 1.78 & 34 & 43.63 & 0.98 \\
FWHM III & 35 & -21.96 & 2.11 & 32 & 43.79 & 1.13 \\
FWHM IV & 17 & -22.39 & 1.93 & 14 & 44.07 & 0.92 \\
\hline
\end{tabular}

\begin{tabular}{l|rcc|rcc}
\hline & \multicolumn{3}{|c|}{$\log v L_{\mathrm{R}}\left[\mathrm{erg} \mathrm{s}^{-1}\right]$} & \multicolumn{3}{c}{$\log L_{\mathrm{FIR}}\left[\mathrm{erg} \mathrm{s}^{-1}\right]$} \\
& $\mathrm{N}$ & $<\log v \mathrm{~L}_{\mathrm{R}}>$ & $\sigma$ & $\mathrm{N}$ & $<\log \mathrm{L}_{\mathrm{FIR}}>$ & $\sigma$ \\
\hline AGN-1 & 68 & 40.12 & 1.84 & 32 & 44.14 & 0.70 \\
XAGN-1 & 29 & 38.84 & 1.20 & 19 & 43.87 & 0.43 \\
VBLG & 39 & 41.06 & 1.66 & 13 & 44.54 & 0.83 \\
FWHM I & 7 & 38.54 & 0.66 & 5 & 43.97 & 0.34 \\
FWHM II & 20 & 39.71 & 1.92 & 12 & 43.88 & 0.49 \\
FWHM III & 25 & 40.21 & 1.93 & 12 & 44.31 & 0.88 \\
FWHM IV & 16 & 41.15 & 1.35 & 3 & 44.72 & 0.67 \\
\hline \hline
\end{tabular}

Da die VBLG überwiegend höhere, die XAGN überwiegend niedrigere Linienbreiten haben, sollten die VBLG statistisch den Halbwertsbreitenintervallen III bzw. IV ähnlich sein, die XAGN hingegen eher den Intervallen I und II. Genau dieses kann anhand der Tabelle 6.3 verifiziert werden. Neben der von der ursprünglichen Selektion der Galaxien abhängigen Einteilung in XAGN und VBLG soll im Folgenden das Hauptaugenmerk auf den unabhängigen, objektiven Parameter FWHM gerichtet werden.

In Tabelle 6.4 sind die Ergebnisse der Korrelationsanalyse der Halbwertsbreite mit den 
Tabelle 6.4: Ergebnisse der Korrelationsanalyse der Linienhalbwertsbreite FWHM mit den Leuchtkräften in den vier Frequenzbändern B, X, FIR und R sowie mit den entsprechenden sechs Flussverhältnissen.

\begin{tabular}{lcrc}
\hline \hline $\mathrm{FWHM} \times$ & $\mathrm{N}$ & \multicolumn{1}{c}{$\mathrm{r}$} & $\mathrm{P}$ \\
\hline $\mathrm{M}_{\mathrm{B}}$ & 101 & -0.22 & 0.001 \\
$\mathrm{LL}_{\mathrm{R}}$ & 68 & 0.40 & 0.001 \\
$\mathrm{~L}_{\mathrm{X}}$ & 94 & 0.21 & 0.039 \\
$\mathrm{~L}_{\mathrm{FIR}}$ & 32 & 0.25 & 0.160 \\
$\mathrm{f}_{\mathrm{R} / \mathrm{B}}$ & 68 & 0.35 & 0.004 \\
$\mathrm{f}_{\mathrm{X} / \mathrm{R}}$ & 62 & -0.27 & 0.034 \\
$\mathrm{f}_{\mathrm{FIR} / \mathrm{R}}$ & 24 & -0.44 & 0.032 \\
$\mathrm{f}_{\mathrm{X} / \mathrm{B}}$ & 93 & 0.10 & 0.328 \\
$\mathrm{f}_{\mathrm{FIR} / \mathrm{B}}$ & 32 & -0.15 & 0.400 \\
$\mathrm{f}_{\mathrm{X} / \mathrm{FIR}}$ & 29 & 0.13 & 0.498 \\
\hline \hline
\end{tabular}

Leuchtkräften in den vier Frequenzbändern B, X, FIR und R sowie mit den entsprechenden Flussverhältnissen aufgelistet.

Bezüglich der vier Frequenzbänder ergab sich eine eindeutig signifikante Korrelation der Halbwertsbreite mit $\mathrm{M}_{\mathrm{B}}$ sowie mit $v \mathrm{~L}_{\mathrm{R}}$. In beiden Fällen ist die Wahrscheinlichkeit der Null-Hypothese P, einen entsprechenden Korrelationskoeffizienten aus einer zufälligen Verteilung zu erhalten, nur $0.1 \%$. $\mathrm{L}_{X}$ korreliert deutlich schwächer mit FWHM $(\mathrm{r}=0.21, \mathrm{P}=0.039)$, während man bei $\mathrm{L}_{\mathrm{FIR}}$ nicht von einer eindeutigen Korrelation sprechen kann, wobei die relativ geringe Anzahl von Datenpunkten $(\mathrm{N}=32)$ berücksichtigt werden muss.

Abbildung 6.7 zeigt die Leuchtkräfte in den vier Frequenzbändern als Funktion der Halbwertsbreite. Zusätzlich sind jeweils Mittelwerte und Standardabweichungen in den vier oben definierten Halbwertsbreitenbereichen I - IV eingezeichnet. Insgesamt betrachtet bestätigen diese Auftragungen und die Ergebnisse der statistischen Analyse diejenigen der Korrelationsanalyse. Allerdings ist der signifikante Unterschied in der Korrelation von $\mathrm{M}_{\mathrm{B}}$ und $\mathrm{L}_{\mathrm{X}}$ mit der Halbwertsbreite anhand der Mittelwerte nicht unmittelbar nachvollziehbar.

Um den in diesen Diagrammen besetzten Parameterraum deutlicher hervorzuheben, wurden empirisch obere und untere Begrenzungen gestrichelt eingezeichnet. Diese wurden so gewählt, dass sie einerseits möglichst nahe an einer maximalen Zahl von Datenpunkten liegen, andererseits aber höchstens ein oder zwei Datenpunkte außerhalb des eingegrenzten Bereichs bleiben. 

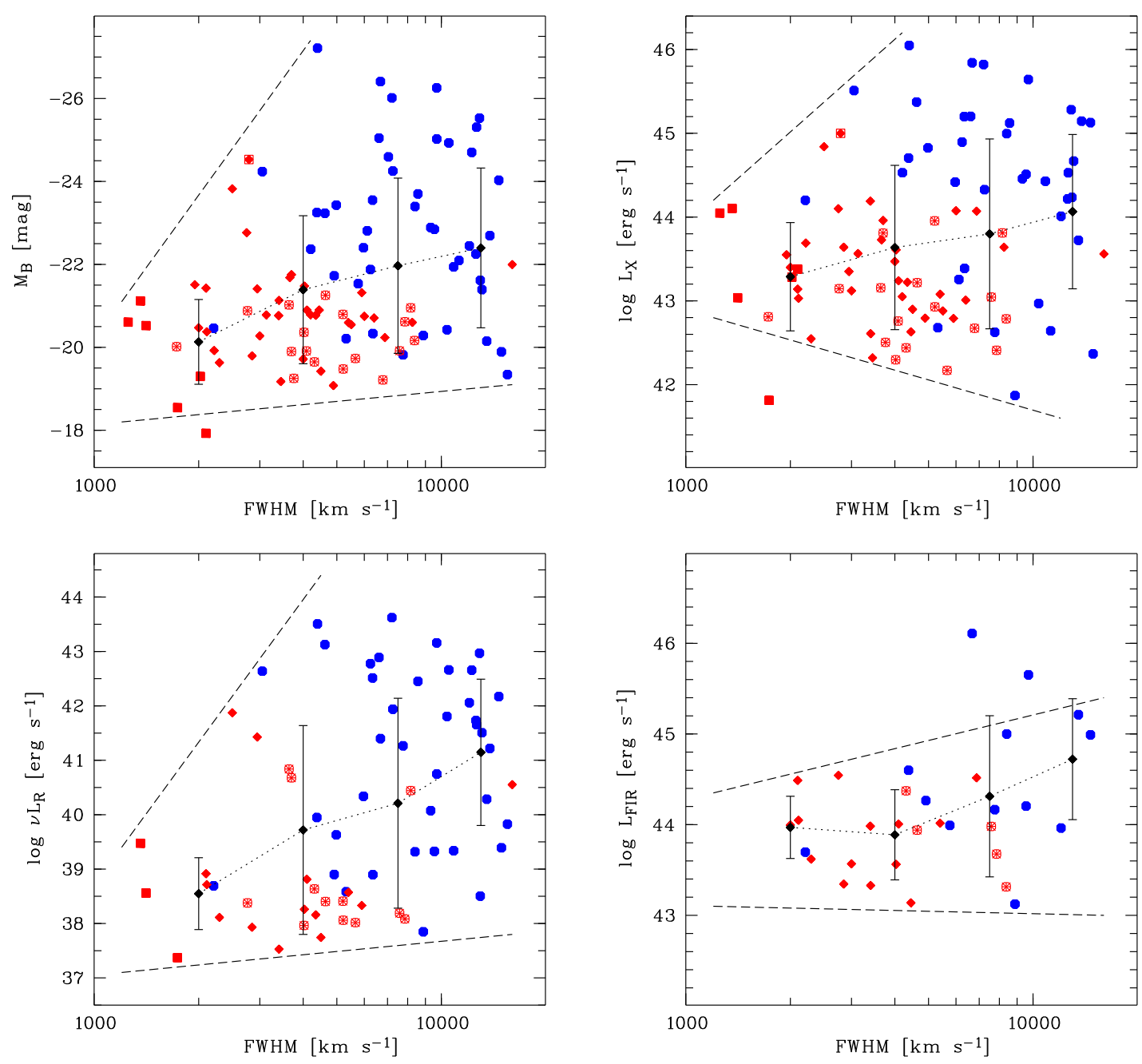

Abbildung 6.7: Leuchtkräfte in den Frequenzbändern B, X, FIR und R als Funktion der Linienhalbwertsbreite FWHM. Zusätzlich sind jeweils Mittelwerte und Standardabweichungen in den vier definierten Halbwertsbreitenbereichen I - IV sowie empirische obere und untere Begrenzungslinien (s. Text) eingezeichnet, die den besetzten Parameterraum verdeutlichen sollen.

Anhand dieser Eingrenzungen ist leichter nachvollziehbar, dass $M_{B}$ stärker als $L_{X}$ mit der Linienbreite FWHM korreliert: in beiden Fällen steigt die obere Begrenzung mit zunehmender Halbwertsbreite an, die untere steigt aber nur bei $\mathrm{M}_{\mathrm{B}}$ leicht an, während sie bei $\mathrm{L}_{X}$ abfällt und somit einer sehr starken Korrelation entgegen steht; es steigt in erster Linie die Streuung der Röntgenleuchtkraft $\mathrm{L}_{X}$ mit der Linienbreite.

Die statistische Analyse in den vier Halbwertsbreitenintervallen bestätigt als unabhängiges Verfahren die Resultate der Korrelationsanalyse. Der Anstieg (bzw. Abfall bei 
$\mathrm{r}<0$ ) der Messgröße mit zunehmender Halbwertsbreite ist dort am größten, wo auch die stärkste Korrelation errechnet wurde $\left(\mathrm{M}_{\mathrm{B}}, v \mathrm{~L}_{\mathrm{R}}\right)$. Zwar sind für die einzelnen Messungen die Dispersion der Datenpunkte und damit die statistischen Unsicherheiten relativ groß (s. Tabelle 6.3 und Abbildung 6.7), so dass ihre Signifikanz - würden sie allein betrachtet - in Frage gestellt werden könnte. In Kombination mit den äquivalenten Ergebnissen der Korrelationsanalyse einerseits wie auch der visuellen Bewertung der entsprechenden Graphen unter Berücksichtigung der Begrenzungslinien andererseits wird die Zuverlässigkeit dieser Resultate entscheidend erhöht. Dadurch, dass im Wesentlichen gleiche Resultate durch drei unabhängige Methoden erzielt wurden, sind sie als stichhaltig einzustufen.

\subsubsection{Flussverhältnisse}

Tabelle 6.5 enthält entsprechend der Tabelle 6.3 die Ergebnisse der statistischen Analyse für die sechs Flussverhältnisse. Wiederum wurde das Gesamtsample AGN-1 einerseits in die Untergruppen XAGN und VBLG, andererseits in die vier definierten Linienbreitenintervalle I - IV separiert. Mittelwerte und Standardabweichungen aus dieser Tabelle sind auch in den nachfolgenden Abbildungen 6.8, 6.9 und 6.10 eingezeichnet.

Bei den Flussverhältnissen $\mathrm{f}_{\mathrm{FIR} / \mathrm{B}}$ und $\mathrm{f}_{\mathrm{X} / \mathrm{B}}$ ist aus der Statistik kein Gang mit der Halbwertsbreite erkennbar. In diesen Fällen kann man daher auch nicht die XAGN bzw. VBLG bestimmten Halbwertsbreitenintervallen zuordnen. Für die übrigen Flussverhältnisse gilt analog wie für die Leuchtkräfte, dass die VBLG statistisch den Halbwertsbreitenintervallen III bzw. IV ähneln, die VBLG hingegen eher den Intervallen I und II.

Betrachtet man die Ergebnisse der Korrelationsanalyse der Linienhalbwertsbreite mit den Verhältnissen der Flüsse in den vier Frequenzbändern (s. Tabelle 6.4), findet sich eine hochsignifikante Korrelation nur für das Flussverhältnis $f_{R / B}$. Die Verhältnisse $f_{X / R}$ und $\mathrm{f}_{\mathrm{FIR} / \mathrm{R}}$ zeigen eine schwächere, aber immer noch signifikante Korrelation mit der Linienbreite. In Abbildung 6.8 sind die entsprechenden Graphiken dargestellt.

Bei den Flussverhältnissen $\mathrm{f}_{\mathrm{X} / \mathrm{FIR}}$ und $\mathrm{f}_{\mathrm{FIR} / \mathrm{B}}$ liefern weder die graphischen Darstellungen (s. Abbildung 6.9) noch die Ergebnisse der Korrelationsanalyse Hinweise auf eine Korrelation mit der Halbwertsbreite. Das kann wiederum auch dadurch bedingt sein, dass in diesen Fällen relativ wenig Datenpunkte vorliegen.

Auch für das Flussverhältnis $\mathrm{f}_{\mathrm{X} / \mathrm{B}}$ findet sich keine Korrelation mit der Halbwertsbreite. Allerdings zeigt hier die graphische Darstellung der Abbildung 6.10 eine deutlich unterschiedliche Verteilung der beiden Untergruppen XAGN und VBLG.

Während die mittleren $\mathrm{f}_{\mathrm{X} / \mathrm{B}}$-Werte für alle vier FWHM-Intervalle nahezu identisch sind, liegt der $\mathrm{f}_{\mathrm{X} / \mathrm{B}}$-Mittelwert für die VBLG etwas höher als für die XAGN. Daraus kann man folgern, dass die VBLG entsprechend im Mittel ein etwas höheres Maß an Aktivität aufweisen (s. Abschnitt 5.1). 
Tabelle 6.5: Statistik der Flussverhältnisse jeweils für das Gesamtsample AGN-1, die beiden Untergruppen XAGN-1 und VBLG sowie für die vier definierten Halbwertsbreitenbereiche I - IV.

\begin{tabular}{l|rcc|ccc}
\hline \hline & \multicolumn{3}{|c|}{$\log \mathrm{f}_{\mathrm{R} / \mathrm{B}}$} & \multicolumn{3}{c}{$\log \mathrm{f}_{\mathrm{X} / \mathrm{R}}$} \\
& $\mathrm{N}$ & $<\log \mathrm{f}_{\mathrm{R} / \mathrm{B}}>$ & $\sigma$ & $\mathrm{N}$ & $<\log \mathrm{f}_{\mathrm{X} / \mathrm{R}}>$ & $\sigma$ \\
\hline AGN-1 & 68 & 1.44 & 1.31 & 62 & -4.76 & 1.18 \\
XAGN-1 & 29 & 0.66 & 0.92 & 28 & -4.32 & 0.87 \\
VBLG & 39 & 2.02 & 1.23 & 34 & -5.13 & 1.29 \\
FWHM I & 7 & 0.57 & 0.33 & 6 & -4.03 & 0.54 \\
FWHM II & 20 & 1.10 & 1.37 & 19 & -4.55 & 1.16 \\
FWHM III & 25 & 1.42 & 1.36 & 24 & -4.79 & 1.19 \\
FWHM IV & 16 & 2.28 & 1.00 & 13 & -5.37 & 1.22 \\
\hline
\end{tabular}

\begin{tabular}{l|rcc|ccc}
\hline & \multicolumn{3}{|c|}{$\log \mathrm{f}_{\mathrm{FIR} / \mathrm{R}}$} & \multicolumn{3}{c}{$\log \mathrm{f}_{\mathrm{X} / \mathrm{FIR}}$} \\
& $\mathrm{N}$ & $<\log \mathrm{f}_{\mathrm{FIR} / \mathrm{R}}>$ & $\sigma$ & $\mathrm{N}$ & $<\log \mathrm{f}_{\mathrm{X} / \mathrm{FIR}}>$ & $\sigma$ \\
\hline AGN-1 & 24 & 1.54 & 1.01 & 29 & -0.68 & 0.65 \\
XAGN-1 & 12 & 2.09 & 0.19 & 19 & -0.86 & 0.51 \\
VBLG & 12 & 0.99 & 1.19 & 10 & -0.35 & 0.78 \\
FWHM I & 4 & 1.93 & 0.25 & 5 & -0.85 & 0.81 \\
FWHM II & 8 & 1.95 & 0.36 & 11 & -0.64 & 0.60 \\
FWHM III & 9 & 1.59 & 0.88 & 10 & -0.72 & 0.62 \\
FWHM IV & 3 & -0.21 & 1.55 & 3 & -0.44 & 0.92 \\
\hline
\end{tabular}

\begin{tabular}{l|rcc|ccc}
\hline & \multicolumn{3}{|c|}{$\log \mathrm{f}_{\mathrm{FIR} / \mathrm{B}}$} & \multicolumn{3}{c}{$\log \mathrm{f}_{\mathrm{X} / \mathrm{B}}$} \\
& $\mathrm{N}$ & $<\log \mathrm{f}_{\mathrm{FIR} / \mathrm{B}}>$ & $\sigma$ & $\mathrm{N}$ & $<\log \mathrm{f}_{\mathrm{X} / \mathrm{B}}>$ & $\sigma$ \\
\hline AGN-1 & 32 & 0.02 & 0.50 & 93 & 0.31 & 0.59 \\
XAGN-1 & 19 & 0.04 & 0.46 & 56 & 0.16 & 0.51 \\
VBLG & 13 & 0.00 & 0.58 & 37 & 0.55 & 0.62 \\
FWHM I & 5 & 0.21 & 0.16 & 13 & 0.39 & 0.61 \\
FWHM II & 12 & -0.14 & 0.45 & 34 & 0.23 & 0.51 \\
FWHM III & 12 & 0.05 & 0.44 & 32 & 0.33 & 0.61 \\
FWHM IV & 3 & 0.27 & 1.16 & 14 & 0.42 & 0.69 \\
\hline \hline
\end{tabular}



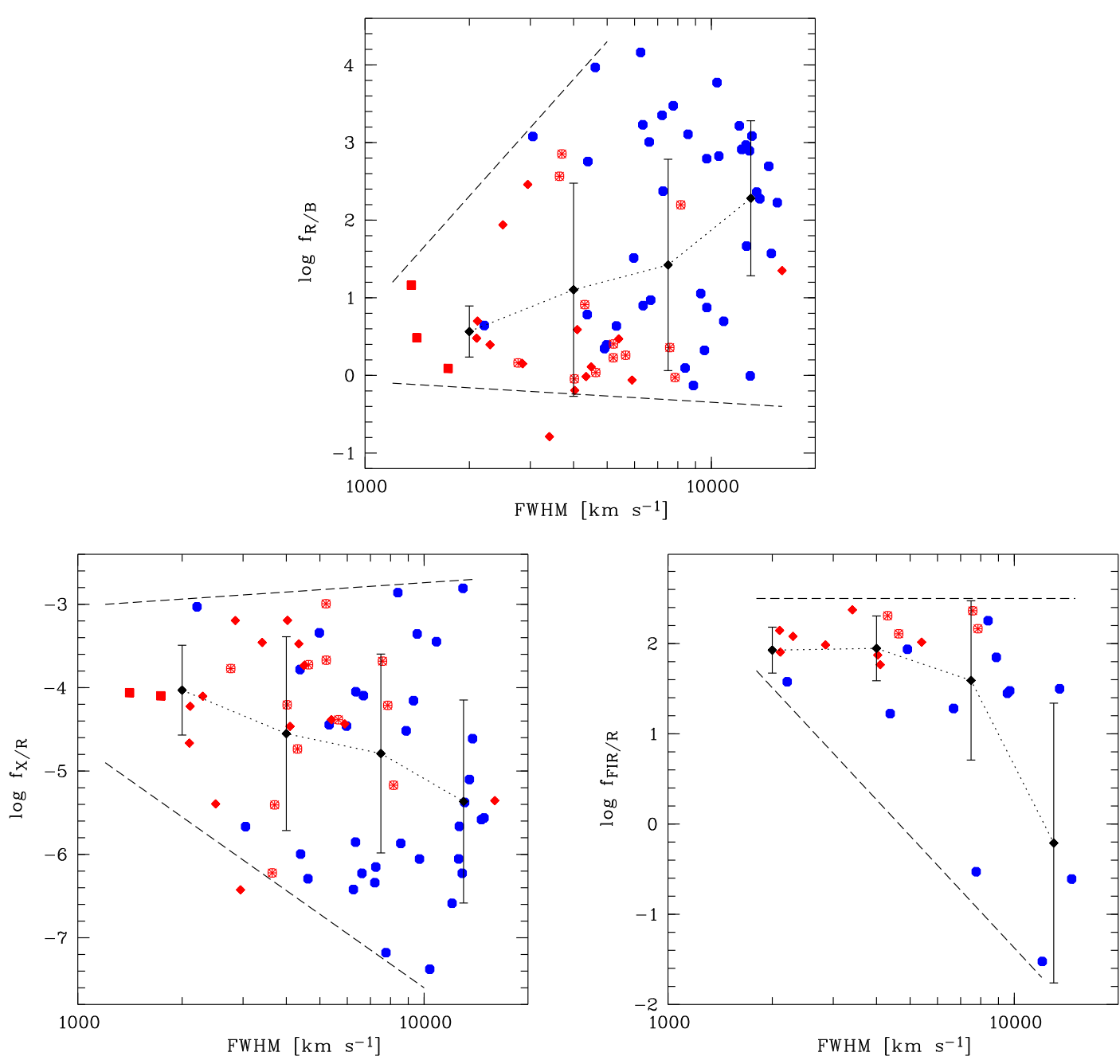

Abbildung 6.8: Die Flussverhältnisse $\mathrm{f}_{\mathrm{R} / \mathrm{B}}, \mathrm{f}_{\mathrm{X} / \mathrm{R}}$ und $\mathrm{f}_{\mathrm{FIR} / \mathrm{R}}$ als Funktion der Linienbreite. Die Mittelwerte und Standardabweichungen in den vier definierten Halbwertsbreitenbereichen sowie empirische obere und untere Begrenzungslinien sind eingezeichnet.

Noch auffälliger als die leicht unterschiedlichen Mittelwerte der beiden Untergruppen ist jedoch, dass sich die VBLG im oberen rechten Teil des Diagramms konzentrieren; VBLG mit niedriger Halbwertsbreite und niedrigen $\mathrm{f}_{\mathrm{X} / \mathrm{B}}$ fehlen im vorliegenden Sample völlig. Um diesen Effekt zu verdeutlichen, wurde in Abbildung 6.10 eine Hilfsgerade so eingezeichnet, dass sich alle VBLG rechts oberhalb die Geraden befinden. Die XAGN hingegen verteilen sich symmetrisch um die Gerade: während 14 Objekte sich in unmittelbarer Nähe der Gerade befinden, liegen jeweils genau 21 XAGN ober- wie auch unterhalb der Geraden. 

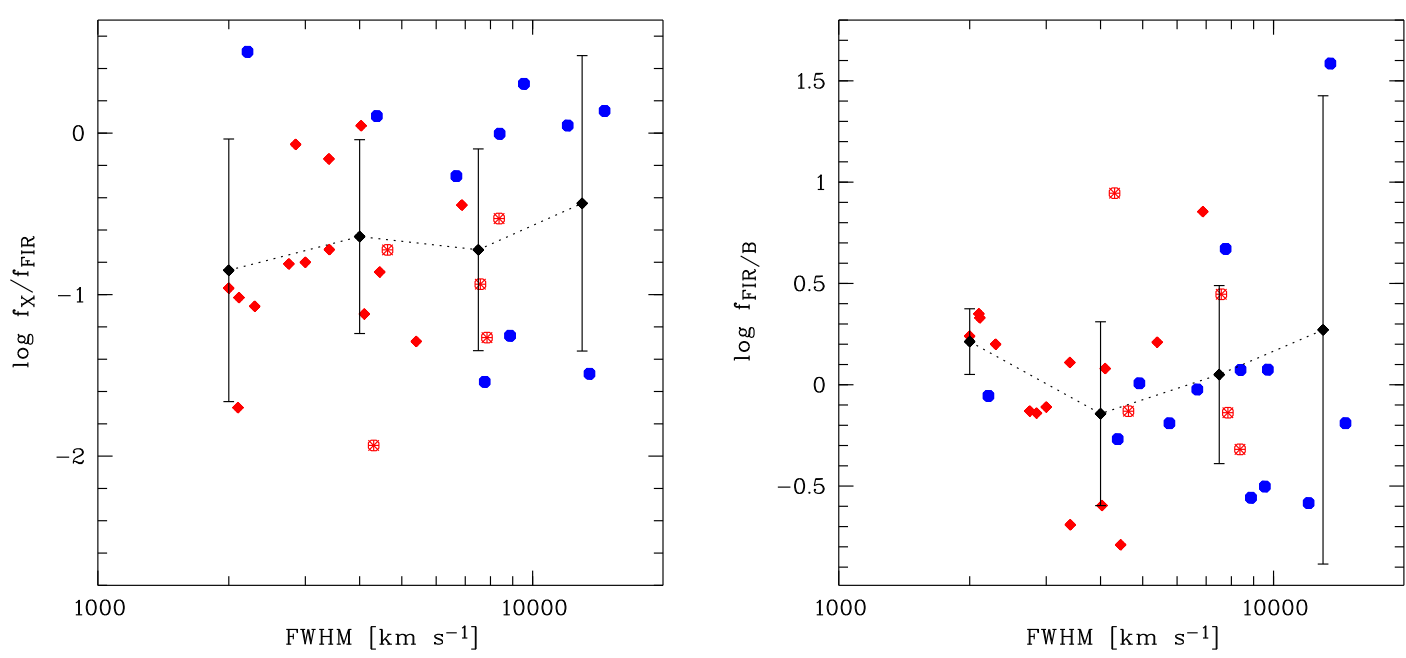

Abbildung 6.9: Die Flussverhältnisse $f_{X / F I R}$ und $f_{F I R / B}$ als Funktion der Linienbreite sowie deren Mittelwerte in den vier definierten Halbwertsbreitenbereichen. Weder diese Auftragung noch die analytische Korrelationsanalyse geben Hinweise auf eine Korrelation dieser Größen. Auf Begrenzungslinien wurde verzichtet, da sie nicht hilfreich erschienen.

Die große Anzahl von Datenpunkten in diesem $\mathrm{f}_{\mathrm{X} / \mathrm{B}}$-FWHM-Diagramm (37 VBLG und 56 XAGN) spricht dafür, dass es sich hierbei um einen systematischen Effekt handelt. In diesem Fall wäre es eine wichtige, einschränkende Beobachtung für ein AGN-Modell und könnte Rückschlüsse auf den Blickwinkel zum AGN zulassen, was im folgenden Kapitel noch genauer diskutiert werden wird (s. Abschnitt 7.3.3).

Auf vergleichbare Beobachtungen von Wills \& Browne (1986) sowie Wills \& Brotherton (1995), die ähnliche Schlussfolgerungen zulassen, wird ebenfalls in Abschnitt 7.3.3 eingegangen.

\subsection{Das Balmerdekrement}

Unter dem Balmerdekrement wird allgemein das Intensitätsverhältnis der Balmerlinien verstanden, im Folgenden bezieht es sich jedoch immer auf die breiten Komponenten der beiden stärksten Balmerlinien $\mathrm{H} \alpha$ und $\mathrm{H} \beta$ (s. Abschnitt 3.1):

$$
B D=\frac{f_{H \alpha}}{f_{H \beta}}
$$

Die Größe dieses Wertes zeigt nur schwache Abhängigkeit von den physikalischen Bedingungen, die in der Linienentstehungsregion herrschen. Unter der Annahme des so ge- 

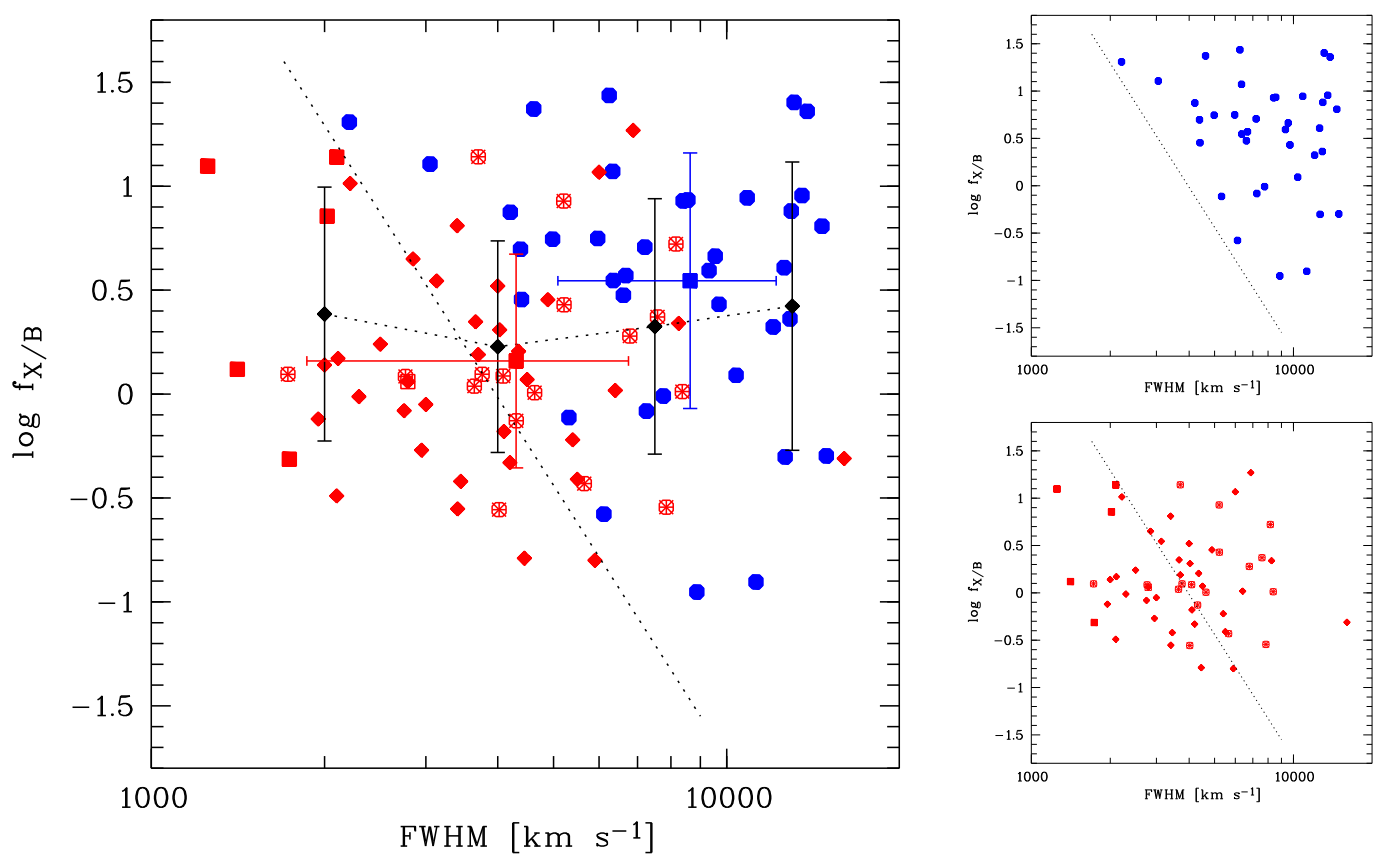

Abbildung 6.10: Aufgetragen ist das Verhältnis des Röntgenflusses zum optischen Fluss $f_{X / B}$ als Funktion der Halbwertsbreite der erlaubten breiten $\mathrm{H} \alpha$ - bzw. $\mathrm{H} \beta$-Emissionslinie. Zusätzlich sind Mittelwerte für die vier definierten Halbwertsbreitenbereiche und die beiden Untergruppen XAGN und VBLG eingezeichnet. Um die unterschiedliche Verteilung der XAGN und VBLG in diesem Diagramm zu verdeutlichen, wurde eine Hilfsgerade so hinzugefügt, dass sich alle VBLG rechts oberhalb befinden. Man erkennt leicht, dass sich die XAGN-1 hingegen weitestgehend symmetrisch um die Gerade verteilen. Die beiden kleinen Graphiken rechts zeigen in identischer Auftragung die Untergruppen VBLG (oben) und XAGN-1 (unten) separat.

nannten ,case B“ der Photoionisationstheorie (optisch dicke Approximation) und einer Temperatur von $10000 \mathrm{~K}$ ergibt sich ein Wert von $B D=2.8$ (Osterbrock 1989).

Weiterhin ist zu berücksichtigten, dass durch die wellenlängenabhängige Staubextinktion das Balmerdekrement vergrößert werden kann und es somit auch ein Maß für die Gesamtextinktion und damit für die Menge des Staubs zwischen Entstehungsgebiet der Linien und Beobachter darstellt. Da ein deutlich geringerer Wert als 2.8 unter plausiblen physikalischen Annahmen nicht möglich ist, wurden für einige Einzelfälle, bei denen solche gemessen wurden, Messungenauigkeiten als Ursache angenommen und im Folgenden ein BD von 2.8 verwendet.

Sowohl die Darstellung des Balmerdekrements BD als Funktion der Linienhalbwertsbreite FWHM in Abbildung 6.11 wie auch die in Tabelle 6.6 zusammengefassten sta- 


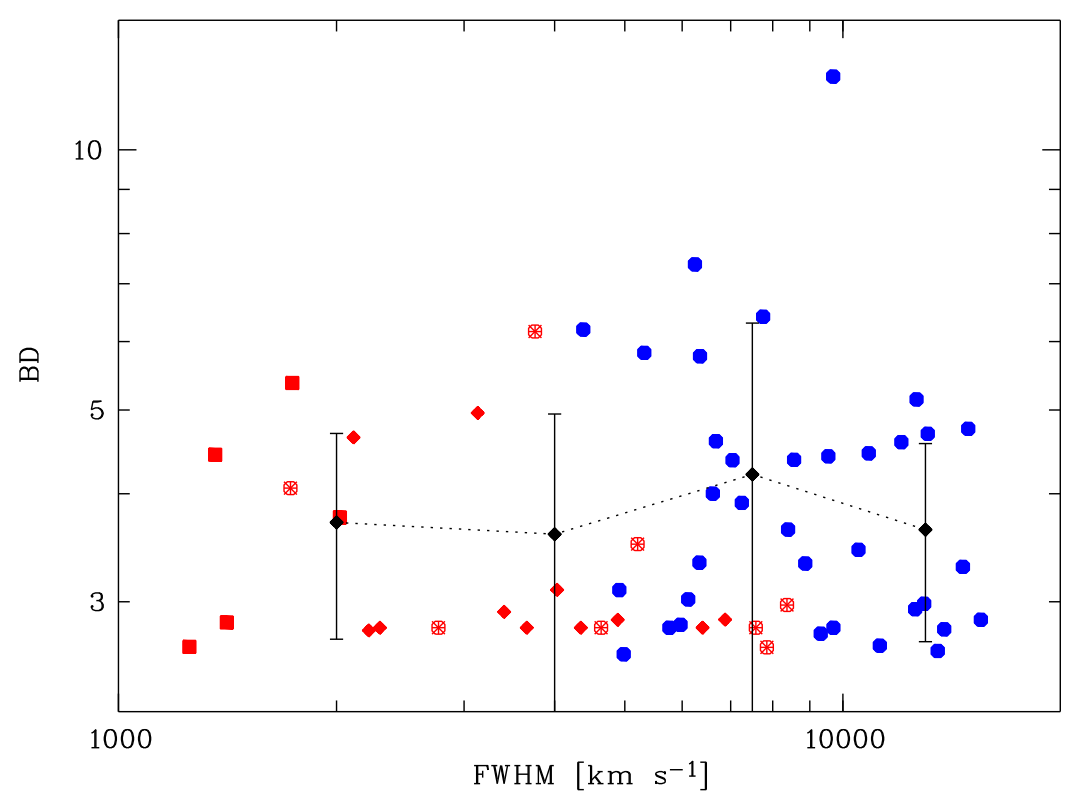

Abbildung 6.11: Balmerdekrement $\mathrm{H} \alpha / \mathrm{H} \beta$ der breiten Emissionslinienkomponenten als Funktion ihrer Halbwertsbreite FWHM. Zusätzlich sind Mittelwerte für die vier definierten FWHM-Intervalle I - IV eingezeichnet (s. auch Tabelle 6.6). Es ist weder ein Zusammenhang zwischen den beiden hier betrachteten Größen noch ein signifikanter Unterschied zwischen den Untergruppen XAGN und VBLG hinsichtlich des Balmerdekrements feststellbar.

Tabelle 6.6: Statistik des Balmerdekrements des Gesamtsamples AGN-1, der beiden Untergruppen XAGN-1 und VBLG sowie der vier Halbwertsbreitenintervalle I - IV.

\begin{tabular}{lcc}
\hline \hline & $\mathrm{N}$ & $\mathrm{BD}$ \\
\hline AGN-1 & 59 & $3.9 \pm 1.6$ \\
XAGN-1 & 24 & $3.4 \pm 1.0$ \\
VBLG & 35 & $4.2 \pm 1.9$ \\
FWHM I & 9 & $3.7 \pm 1.0$ \\
FWHM II & 12 & $3.6 \pm 1.4$ \\
FWHM III & 25 & $4.2 \pm 2.1$ \\
FWHM IV & 13 & $3.6 \pm 0.9$ \\
\hline \hline
\end{tabular}


Tabelle 6.7: Ergebnisse der Korrelationsanalyse des Balmerdekrements mit der Linienhalbwertsbreite FWHM, mit den Leuchtkräften in den vier Frequenzbändern B, X, FIR und R sowie mit deren Flussverhältnissen. Nur die Radioleuchtkraft $v L_{R}$ und die drei entsprechenden Flussverhältnisse zeigen eine gewisse Korrelation mit dem Balmerdekrement.

\begin{tabular}{lcrc}
\hline \hline & $\mathrm{N}$ & \multicolumn{1}{c}{$\mathrm{r}$} & $\mathrm{P}$ \\
\hline FWHM & 59 & 0.03 & 0.825 \\
$\mathrm{M}_{\mathrm{B}}$ & 59 & -0.08 & 0.533 \\
$\mathrm{~L}_{\mathrm{X}}$ & 53 & 0.12 & 0.398 \\
$\mathrm{~L}_{\mathrm{FIR}}$ & 21 & 0.16 & 0.490 \\
$\mathrm{LL}_{\mathrm{R}}$ & 44 & 0.33 & 0.031 \\
$\mathrm{f}_{\mathrm{R} / \mathrm{B}}$ & 44 & 0.37 & 0.014 \\
$\mathrm{f}_{\mathrm{FIR} / \mathrm{R}}$ & 18 & -0.56 & 0.016 \\
$\mathrm{f}_{\mathrm{X} / \mathrm{R}}$ & 39 & -0.36 & 0.026 \\
$\mathrm{f}_{\mathrm{X} / \mathrm{FIR}}$ & 18 & 0.37 & 0.132 \\
$\mathrm{f}_{\mathrm{FIR} / \mathrm{B}}$ & 21 & -0.22 & 0.327 \\
$\mathrm{f}_{\mathrm{X} / \mathrm{B}}$ & 52 & -0.04 & 0.756 \\
\hline \hline
\end{tabular}

tistischen Werte zeigen, dass das mittlere Balmerdekrement der VBLG zwar etwas über dem der XAGN-1 liegt, dieser Unterschied aber statistisch nicht signifikant ist. Die Unterschiede zwischen den vier Halbwertsbreitenbereichen sind noch geringer und bewegen sich ausschließlich im Rahmen der Streuung der Messwerte. Es gibt keinerlei Korrelation zwischen Balmerdekrement und Linienbreite.

Wie in Abschnitt 2.1 dargelegt, wurde im Rahmen des „Unified Models“ häufiger postuliert, dass BLRG bzw. VBLG ein höheres Balmerdekrement als gewöhnliche AGN zeigen - damit kommt obigen Ergebnissen unseres Sample besondere Bedeutung zu.

Wäre die Halbwertsbreite ein direktes Maß des Inklinationswinkels, unter dem wir eine z.B. scheibenförmige BLR sehen, und wäre das Balmerdekrement - verursacht durch unterschiedliche Staubsäulendichten entlang des Sehstrahls - ebenfalls vom Inklinationswinkel abhängig, so würde man eine Korrelation der beiden Größen erwarten. Da dies nicht der Fall ist, sind die Annahmen zumindest in dieser einfachen Form nicht zutreffend.

Abbildung 6.12 zeigt das Balmerdekrement als Funktion der Leuchtkräfte in den vier Frequenzbändern B, X, FIR und R, in Abbildung 6.13 ist es als Funktion der sechs ent- 

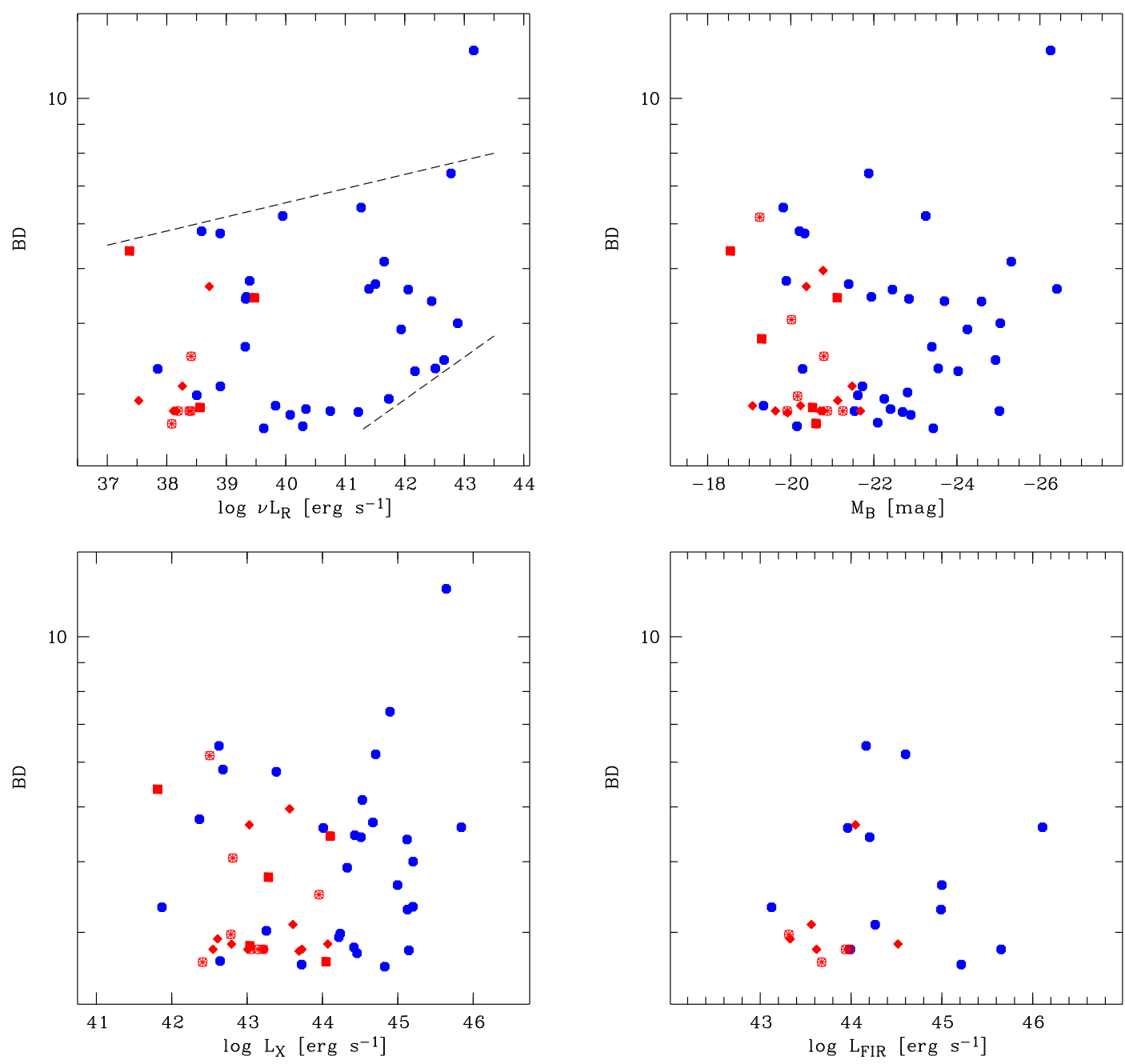

Abbildung 6.12: Das Balmerdekrement als Funktion der Leuchtkräfte in den vier betrachteten Frequenzbändern. Eine mäßig starke Korrelation besteht nur zwischen Radioleuchtkraft $\mathrm{vL}_{\mathrm{R}}$ und Balmerdekrement $(\mathrm{r}=0.33, \mathrm{P}=0.031$; siehe Tabelle 6.7), die anderen drei Leuchtkräfte sind nicht mit dem Balmerdekrement korreliert.

sprechenden Flussverhältnisse aufgetragen. In Tabelle 6.7 sind die korrespondierenden Ergebnisse der Korrelationsanalyse zusammengefasst.

Von den vier Leuchtkräften scheint einzig $v L_{R}$ eine gewisse Korrelation mit dem Balmerdekrement aufzuweisen - dafür spricht der Korrelationskoeffizient von $r=0.33$ bzw. $\mathrm{P}=0.031$. Die anderen drei Leuchtkräfte sind eindeutig nicht mit dem Balmerdekrement korreliert. Dieses Resultat wird bei Betrachtung der Flussverhältnisse bestätigt: die drei mit dem Radiofluss gebildeten Verhältnisse $\left(\mathrm{f}_{\mathrm{R} / \mathrm{B}}, \mathrm{f}_{\mathrm{FIR} / \mathrm{R}}, \mathrm{f}_{\mathrm{X} / \mathrm{R}}\right)$ korrelieren mit 
dem Balmerdekrement, die drei anderen Flussverhältnisse $\left(f_{X / F I R}, f_{R / B}, f_{X / B}\right)$ korrelieren hingegen nicht.

Diese Konstellation deutet sehr stark darauf hin, dass die bei den drei Flussverhältnissen gefundene Korrelation nur sekundärer Natur ist und allein durch die primäre Korrelation der Radioleuchtkraft mit dem Balmerdekrement hervorgerufen wird.

Da im Radiodatensatz die VBLG dominieren, wird auch die Korrelation des Balmerdekrements mit der Radioleuchtkraft von diesem Teilsample geprägt. Betrachtet man die beiden Teilsample VBLG und XAGN separat, läßt sich keine signifikante Korrelation einer der hier diskutierten Größen mit dem Balmerdekrement mehr feststellen. Dies konnte erwartet werden, da schon die Korrelationen bezogen auf das Gesamtsample nicht sehr stark sind. Daher erscheint an dieser Stelle eine weitergehende separate Erörterung der beiden Teilsamples nicht sinnvoll.

\subsection{Ergebnisse der optischen Spektroskopie}

Die in diesem Abschnitt erzielten Ergebnisse stützen sich auf drei unabhängige Methoden: statistische Analyse, Korrelationsanalyse sowie graphische Darstellung und Bewertung. Da alle drei Methoden im Wesentlichen jeweils zu gleichen Resultaten führen, erhöht dies die Aussagekraft der Ergebnisse entscheidend.

In Bezug auf die Untersuchungen zur Linienhalbwertsbreite bleibt festzustellen, dass genau die drei Flussverhältnisse, in die der Radiofluss eingeht, mit der Linienbreite korrelieren. Das ist konsistent mit dem ebenfalls hier gefundenen Ergebnis, dass auch die Radioleuchtkraft stark mit der Linienbreite korreliert.

Andererseits korreliert zwar die optische Helligkeit stark mit der Linienbreite, diejenigen Verhältnisse, die mit dem optischen Fluss gebildet wurden, weisen jedoch keine vergleichbare Korrelation mit der Linienbreite auf. Daraus lässt sich folgern, dass die Radioleuchtkraft, nicht jedoch die optische Leuchtkraft, über die Leuchtkräfte in den anderen Frequenzbändern dominiert.

Besonders wichtig unter den Flussverhältnissen ist $f_{X / B}$ : einerseits kann es als Maß für die nichtthermische Aktivität angesehen werden, andererseits zeigt es deutliche Diskrepanzen zwischen XAGN und VBLG, die nicht allein auf einen Effekt unterschiedlicher Linienbreiten zurückgeführt werden können.

Bezüglich des Balmerdekrements zeigte sich keinerlei Zusammenhang mit der Linienhalbwertsbreite. Von den Leuchtkräften der vier Frequenzbereiche ergab sich nur für die Radioleuchtkraft eine mäßig starke Korrelation mit dem Balmerdekrement, die offensichtlich wiederum dazu führt, dass auch die drei Flussverhältnisse, in die die Radioleuchtkraft eingeht, mit dem Balmerdekrement korrelieren, die anderen drei hingegen nicht. 

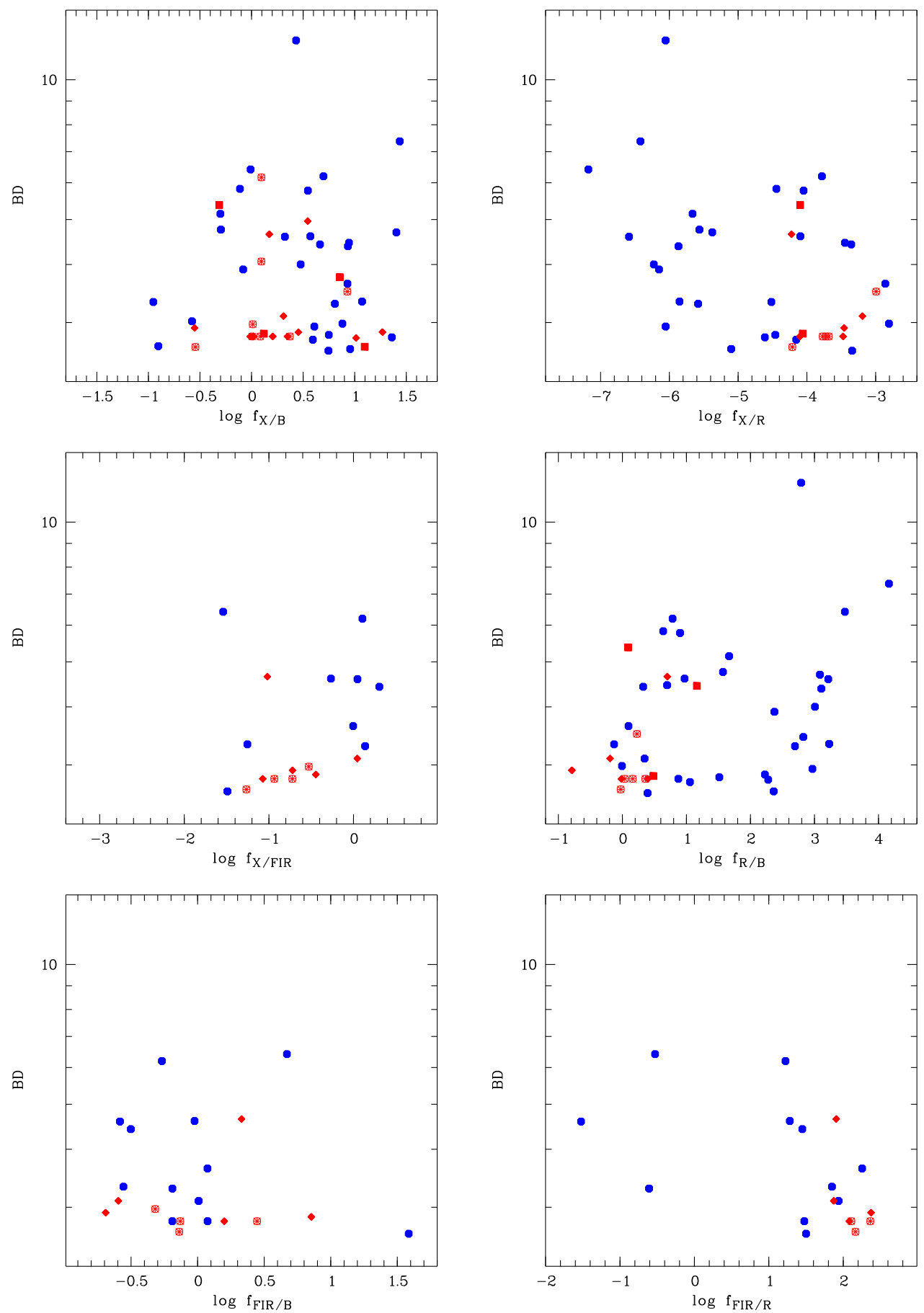

Abbildung 6.13: Balmerdekrement als Funktion der sechs Flussverhältnisse. Nach der numerischen Korrelationsanalyse (s. Tabelle 5.6) sind die drei Verhältnisse, in die der Radiofluss eingeht, mit dem Balmerdekrement korreliert (rechte Spalte dieser Abbildung), die anderen drei hingegen nicht (linke Spalte). 
Ein signifikanter Unterschied zwischen den Untergruppen XAGN und VBLG hinsichtlich des Balmerdekrements oder einer seiner Korrelationen konnte nicht festgestellt werden. 


\title{
Kapitel 7
}

\section{Zusammenfassung und Diskussion der Ergebnisse}

\begin{abstract}
Abschließend sollen die in den vorangegangenen Kapiteln erarbeiteten Ergebnisse zusammengefasst und insbesondere auch gemeinsam diskutiert werden, um ein über die Einzelresultate hinaus gehendes, konsistentes Bild der untersuchten Aktiven Galaxien zu erhalten.
\end{abstract}

\subsection{Röntgenselektion Aktiver Galaxien}

Der erste große Schritt für diese Arbeit war die Selektion des XAGN-Samples. Wie in Abschnitt 2.2 beschrieben, wurden diese zuvor nicht als aktiv klassifizierten Galaxien aufgrund einer hohen Zählrate im Röntgenkatalog RASS-BSC ausgewählt.

In einem zweiten Schritt wurden die Quellen per optischer Spektroskopie klassifiziert. Dabei ergab sich, dass die Selektionsstrategie sehr erfolgreich war und überwiegend bisher unbekannte Aktive Galaxien identifizierte.

Um die Effizienz der Selektion zu quantifizieren, sind in Tabelle 7.1 in Anlehnung an Tabelle 3.3 die absoluten und relativen Häufigkeiten der einzelnen Galaxien-Typen nur für das XGAL-Teilsample aufgelistet. Das Verfahren hat zu einer sehr hohen Detektionsquote von $79 \%$ AGN geführt.

Es zeigte sich ferner, dass im XGAL-Sample vier größere Gruppen von Galaxien enthalten sind: Typ-1 AGN, Übergangstypen Seyfert-1.5 ... 1.9, Seyfert-2/LINER/LLAGN und inaktive Galaxien (s. Tabelle 7.1 bzw. Abbildung 3.4). Andere AGN-Typen (BL Lac) spielen anteilsmäßig keine Rolle. 
Tabelle 7.1: Übersicht über die absoluten und prozentualen Häufigkeiten der einzelnen Galaxien-Typen für das XGAL-Teilsample zur Quantifizierung der Effizienz der Röntgenselektion. Die Bezeichnungen entsprechen denjenigen der Tabelle 3.3. Da die Prozentzahlen auf ganze Zahlen gerundet sind, ergeben sie in der Summe nicht zwangsläufig exakt $100 \%$.

\begin{tabular}{|c|c|c|c|c|c|c|c|c|}
\hline Тур & $\begin{array}{l}\text { Häu } \\
\text { abs. }\end{array}$ & $\begin{array}{l}\text { igkeit } \\
\text { rel. }\end{array}$ & Gruppe & $\begin{array}{l}\text { Häu } \\
\text { abs. }\end{array}$ & $\begin{array}{r}\text { gkeit } \\
\text { rel. }\end{array}$ & Klasse & $\begin{array}{l}\text { Häu } \\
\text { abs. }\end{array}$ & $\begin{array}{r}\text { gkeit } \\
\text { rel. }\end{array}$ \\
\hline BL Lac & 4 & $4 \%$ & BL Lac & 4 & $4 \%$ & BL Lac & 4 & $4 \%$ \\
\hline $\begin{array}{l}\text { QSO } \\
\text { Sy1 } \\
\text { NLS1 }\end{array}$ & $\begin{array}{r}1 \\
33 \\
6\end{array}$ & $\begin{array}{r}1 \% \\
30 \% \\
5 \% \\
\end{array}$ & Typ-1 AGN & 40 & $36 \%$ & \multirow{3}{*}{$\begin{array}{l}\text { Typ-1,2,3 } \\
\text { AGN }\end{array}$} & \multirow{3}{*}{83} & \multirow{3}{*}{$75 \%$} \\
\hline $\begin{array}{l}\text { Sy } 1.5 \\
\text { Sy1.8 } \\
\text { Sy1.9 }\end{array}$ & $\begin{array}{l}8 \\
2 \\
7\end{array}$ & $\begin{array}{l}7 \% \\
2 \% \\
6 \%\end{array}$ & Sy $1.5 \ldots 1.9$ & 17 & $15 \%$ & & & \\
\hline $\begin{array}{l}\text { Sy2 } \\
\text { LLAGN } \\
\text { LINER }\end{array}$ & $\begin{array}{r}6 \\
17 \\
3\end{array}$ & $\begin{array}{r}5 \% \\
15 \% \\
3 \%\end{array}$ & $\begin{array}{l}\text { Sy2/LINER/ } \\
\text { LLAGN }\end{array}$ & 26 & $24 \%$ & & & \\
\hline $\begin{array}{l}\text { H II } \\
\text { inaktiv }\end{array}$ & $\begin{array}{r}3 \\
20 \\
\end{array}$ & $\begin{array}{r}3 \% \\
18 \% \\
\end{array}$ & $\begin{array}{l}\text { H II } \\
\text { inaktiv }\end{array}$ & $\begin{array}{r}3 \\
20 \\
\end{array}$ & $\begin{array}{r}3 \% \\
18 \% \\
\end{array}$ & $\begin{array}{l}\text { H II } \\
\text { inaktiv }\end{array}$ & $\begin{array}{r}3 \\
20 \\
\end{array}$ & $\begin{array}{r}3 \% \\
18 \% \\
\end{array}$ \\
\hline \multicolumn{3}{|c|}{110} & \multicolumn{3}{|c|}{110} & \multicolumn{3}{|c|}{110} \\
\hline
\end{tabular}

\subsubsection{Röntgenemission Elliptischer Galaxien}

Dass auch einige nicht aktive Galaxien mit unserem Verfahren selektiert wurden, kann nicht wirklich überraschen. Es handelt sich dabei überwiegend um elliptische Galaxien bzw. so genannte „frühe Hubble-Typen“. Durch unsere Auswahl haben wir einige besonders röntgenleuchtkräftige Exemplare $\left(\mathrm{L}_{\mathrm{X}} \approx 10^{42} \ldots 10^{44} \mathrm{erg} \mathrm{s}^{-1}\right)$ gefunden.

Durch Röntgenbeobachtungen elliptischer Galaxien mit den Satellitenteleskopen EINSTEIN, vor allem aber auch ROSAT, hat man entgegen früherer Erwartungen gelernt, dass diese große Mengen heißen Gases enthalten können. Dieses Gas weist Temperaturen von etwa 10 Millionen Kelvin auf und strahlt kontinuierlich Röntgenstrahlung (Bremsstrahlung) ab. Es verleiht den Galaxien große „Röntgenhalos“, deren Ausdehnung typischerweise weit über den optisch sichtbaren Bereich der Galaxien hinausreicht. 
Die Herkunft dieses heißen Gases ist noch nicht sicher geklärt. Nach einer gängigen Deutung handelt es sich um ein Nebenprodukt früherer Sternentstehung: Im Laufe ihrer Entwicklung stoßen die meisten Sterne einen Teil ihrer Hülle durch Explosionen oder so genannte Sternenwinde ab. Die massereichsten Sterne setzten hierbei soviel Energie frei, dass sie dieses Material weit in die Galaxie hinausschleudern können, wo es später durch Stoßwellen von Supernova-Explosionen aufgeheizt wird (Fabbiano et al. 1992, Beuing et al. 1999, Matsushita et al. 1999, O’Sullivan et al. 2001).

Dass es elliptische Galaxien mit besonders hohen Röntgenleuchtkräften von $\mathrm{L}_{X}>10^{43.5}$ $\mathrm{erg} \mathrm{s}^{-1}$ gibt, wurde bereits von Stocke et al. (1991) gefunden, die diese als ,,cooling flow galaxies" bezeichneten.

Da die inaktiven Galaxien des Samples nicht Gegenstand dieser Arbeit sind, wird auf sie hier nicht näher eingegangen.

\subsubsection{Röntgenemission Aktiver Galaxien}

Die größte Gruppe im XAGN-Teilsample bilden mit 36\% die Typ-1 AGN. Das ist keinesfalls überraschend, sondern im Gegenteil Ausdruck für die Effizienz unserer Selektionsstrategie. Ein wesentliches Ergebnis dieser Arbeit ist allerdings, dass auch die Übergangstypen Seyfert-1.5..1.9 mit 15\% sowie die Gruppe Seyfert-2/LINER/ LLAGN mit $25 \%$ ebenfalls stark vertreten sind.

\section{Seyfert-1}

Die Röntgenspektren von Seyfert-1/Typ-1 Galaxien lassen sich durch ein Potenzgesetz beschreiben (s. Abschnitt 4.4.3 und Gleichung 4.8). Hasinger et al. (1991) und Walter \& Fink (1993) haben mit ROSAT-PSPC Beobachtungen einen Photonenindex von $\Gamma=2.3$ für Seyfert-1 Galaxien gefunden. Im weichen Röntgenbereich unterhalb von etwa $1 \mathrm{keV}$ wurde häufig ein zusätzlicher Exzess beobachtet (Turner \& Pounds 1989, Walter \& Fink 1993). Generell zeigen Seyfert-1 Galaxien keine signifikanten internen Säulendichten von Neutralgas, die über den galaktischen Vordergrund hinausgehen $\left(\mathrm{N}_{H}<10^{20} \mathrm{~cm}^{-2}\right)$ (Gondhalekar et al. 1997, Laor et al. 1997). Das geht einher mit der vom „Unified Model“" propagierten unabsorbierten Sicht auf das eigentliche Zentrum des AGN.

\section{Seyfert-2}

Dementsprechend sollte im Rahmen dieses Modells bei Seyfert-2 Galaxien der Kern und damit auch die Röntgenstrahlung verdeckt bzw. absorbiert sein. Tatsächlich wurden hohe Säulendichten von $10^{22} \mathrm{~cm}^{-2}$ bis über $10^{24} \mathrm{~cm}^{-2}$ beobachtet (Malizia et al. 1997, 
Sambruna et al. 1999). Das bedeutete, dass diese Quellen im weichen Röntgenbereich mit ROSAT nicht beobachtbar wären. Andererseits kann es auch noch eine zusätzliche weiche Röntgenkomponente geben, die auf Reflexion an der Scheibe oder dem Torus zurückgeführt wird (Smith \& Done 1996).

Wie in Abschnitt 1.3 erläutert, werden im „Unified Model“ die Übergangstypen Seyfert1.5... 1.9 mit partieller Absorption entlang des Sehstrahls erklärt, der die optische BLREmission und die Röntgenemission gleichermaßen betreffen sollte.

\section{LINER}

Die Natur von LINER Galaxien, die ursprünglich von Heckman (1980) definiert wurden (s. Abschnitt 3.2), und ihre Anregungsmechanismen wurden sehr kontrovers diskutiert und sind nach wie vor nicht endgültig geklärt (Filippenko 2003). Geklärt ist inzwischen hingegen, dass zumindest ein Teil der LINER durch Aktive Kerne angeregt wird, die allerdings leuchtschwächer als diejenigen in Seyfert-Galaxien sind. Das haben vor allem Beobachtungen von schwachen, breiten H $\alpha$-Linienkomponenten (Ho et al. 1997b, Barth et al. 1999a,b) und auch von punktförmigen Röntgenquellen auf hochaufgelösten HRIBildern (Komossa et al. 1999, 2000) gezeigt.

\section{LLAGN}

Aufgrund ihrer geringen Leuchtkraft werden diese schwachen Aktiven Kerne als „lowluminosity AGN“ (LLAGN) bezeichnet (Ho, Filippenko \& Sargent 1997a). Sie sind in den letzten Jahren auch deswegen in den Fokus der AGN-Forschung gerückt, weil sich immer deutlicher gezeigt hat, dass sie die Mehrheit der AGN-Population stellen (Barth 2002).

Neben 3\% LINER und 5\% Seyfert-2 Galaxien haben wir mit 15\% deutlich mehr LLAGN in unserem röntgenselektierten Sample. Aufgrund ihres optischen Linienspektrums könnten die LLAGN formal auch Seyfert-2 oder LINER sein - die Emissionslinien sind zu schwach, um diese Unterscheidung sicher zu treffen.

Sowohl aufgrund dieser beobachtungstechnischen Abgrenzungsschwierigkeiten wie auch der oben geschilderten, nicht letztlich geklärten physikalischen Unterschiede ist es sinnvoll, die Gruppe Sy2/LINER/LLAGN gemeinsam als das leuchtschwache Ende der AGN-Sequenz zu betrachten, die dann mit 24\% den immerhin zweitgrößten Anteil des XAGN-Teilsamples stellt.

Der genaue Mechanismus der Röntgenemission bei LLAGN und LINER-Galaxien ist noch Gegenstand laufender Diskussionen. Es werden abweichend von der klassischen 
Vorstellung von optisch dicken, geometrisch dünnen Akkretionsscheiben so genannte ADAFs (,advection dominated accretion flows") bzw. LDAFs (,low-radiative efficiency accretion flows") vorgeschlagen, die mit einer geringen Akkretionsrate einhergehen (Quataert et al. 1999, Quataert 2001, Shields et al. 2001, Ho 2002, 2003).

Auch Awaki et al. (2001) kommen anhand von Röntgenbeobachtungen zu dem Schluss, dass diese Quellen zwar ein supermassives Schwarzes Loch enthalten, aufgrund einer kleinen Akkretionsrate aber nur eine geringe Kernleuchtkraft entwickeln. Eine größere Untersuchung von 21 LINER und 17 LLAGN haben Terashima et al. (2002) mit dem japanischen Röntgensatelliten ASCA durchgeführt. Sie beschreiben die Röntgenspektren ihrer Quellen mit der Kombination einer weichen, thermischen Komponente und einer harten Komponente, die dem Potenzgesetz für AGN entspricht (s.o.). Die physikalische Interpretation ihrer Ergebnisse haben sie für eine spätere Arbeit angekündigt.

\subsubsection{Schlussfolgerungen aus der selektierten Population}

Die Typ-1 Galaxien stellen die größte Gruppe unter den XAGN. Dies überrascht nicht, da das „Unified Model“ für Typ-1 Galaxien einen freien Blicks auf das Zentrum vorsieht, so dass uns die zentrale Röntgenstrahlung direkt und ungehindert erreichen kann.

Der hohe Anteil von LLAGN und LINER-Galaxien von zusammen 18\% im röntgenselektierten Sample unterstreicht, dass es sich bei diesen Typen tatsächlich um AGN handelt.

Der sehr hohe Anteil von LINER/LLAGN, Seyfert-2 und den Übergangstypen Seyfert1.5. . 1.9 von zusammengenommen 39\% im XAGN-Sample impliziert, dass ihre Röntgenstrahlung nicht sehr stark absorbiert bzw. abgeschattet sein kann. Dieses widerspricht einfachen Vorstellungen, die davon ausgehen, dass bei diesen Typen der eigentliche Kern entweder vollständig verdeckt oder seine Emission zumindest durch den Staubtorus teilabsorbiert ist. Es unterstützt hingegen die Hypothese von einer zusätzlichen Röntgenkomponente, die uns z.B. reflektiert erreicht (Smith \& Done 1996) oder aber einem inneren, dicken Torus entstammt, der von der äußeren dünnen Scheibe nicht vollständig verdeckt wird (Shields et al. 2001).

Theoretisch denkbar wäre noch, dass Röntgenemission in Folge starker Sternentstehung einen signifikanten Beitrag in unseren Quellen liefert. Warum dies nicht der Fall ist, wird im Folgenden noch diskutiert werden. Dass sich nur drei H II-Galaxien unter den röntgenselektierten XGAL befinden, ist bereits ein starkes Indiz hierfür.

Eine Alternative zum absorbierenden Torus im „Unified Model“ haben Malkan et al. (1998) vorgeschlagen. Sie fanden auf HST-Bildern naher Seyfert-Galaxien, dass Seyfert-2 Galaxien mit höherer Wahrscheinlichkeit als Seyfert-1 Galaxien irreguläre und gestörte Staubabsorption in ihren Zentren wie auch galaktische Staubstreifen zeigen. Daher schlugen sie vor, dass die Absorption der BLR-Emission in Seyfert-2 
Galaxien nicht auf einem zentralen Torus basiert, sondern auf galaktischen Staubstreifen, die sich in Abständen von typischerweise mehr als 100 pc vom Zentrum befinden.

Träfe diese Vorstellung zu, hätte sie etliche Konsequenzen: durch den viel größeren Abstand gäbe es kaum eine Wechselwirkung zwischen der zentralen Quelle und dem absorbierenden Staub. Insbesondere müsste es auch keine gleichgerichtete Orientierung von zentraler Quelle und absorbierenden Strukturen geben, wie sie im Rahmen des „Unified Models" häufig angenommen wird und teilweise auch bereits beobachtet wurde (s. Kapitel 1). Daraus wiederum folgte, dass die äußere Erscheinung von der Orientierung der Staubstreifen, nicht jedoch zwangsläufig von der Orientierung der zentralen Quelle abhinge.

\subsection{Multifrequenzdatensatz und -analyse}

Wie bereits in Kapitel 1 diskutiert, ist es aufgrund der großen Vielfalt Aktiver Galaxien und ihres komplexen Aufbaus von entscheidender Bedeutung, sie möglichst multispektral zu untersuchen (siehe auch Wills 1999, Véron-Cetty \& Véron 2000, Collin 2001). So beobachtet man z.B. in unterschiedlichen Spektralbereichen Emission aus unterschiedlichen Raumgebieten, was im Hinblick auf die Verifikation der Modellvorstellungen im Rahmen des „Unified Models“ extrem wichtig ist. Daher werden in dieser Arbeit neben den optischen Spektren auch die Kontinuumsflüsse aus den Frequenzbändern Optisch, Röntgen, Radio und Ferninfrarot analysiert. Letzteres ist insbesondere auch deswegen sehr wichtig, weil auf die Weise die röntgenselektierten Galaxien in Spektralbereichen untersucht werden können, die nicht zu ihrer Selektion dienten.

\subsubsection{Der Datensatz}

Mit Hilfe etlicher großer Galaxienkataloge, deren Eigenschaften in Kapitel 4 ausführlich besprochen wurden, haben wir unseren Datensatz zusammengestellt (Pietsch, Bischoff, et al. 1998). Im Optischen und im Röntgenbereich ist der Datensatz nahezu vollständig, was zum einen durch die Qualität der entsprechenden Kataloge, zum anderen natürlich auch durch das Selektionsverfahren der Quellen begründet ist. Optische Helligkeiten liegen für alle Galaxien des Samples vor, Röntgenflüsse sind nur für sieben VBLG nicht verfügbar. Radiodaten liegen für $61 \%$, Ferninfrarotdaten für $27 \%$ des Gesamtsamples vor. Diese unterschiedlichen Umfänge der Datensätze in den verschiedenen Frequenzbändern müssen stets berücksichtigt werden.

Insbesondere bei den beiden letztgenannten, kleineren Datensätzen wurde die Vollständigkeit auch für die verschiedenen AGN-Typen einzeln untersucht und diskutiert (siehe 
Abbildungen 4.7 und 4.10). Wie erwartet liegen für einen höheren Anteil der VBLG und Typ-1 XAGN Radio- und FIR-Daten vor als für die nicht aktiven Galaxien.

Aus den den Katalogen entnommenen Flüssen wurden unter Annahme des in Abschnitt 4.2 skizzierten kosmologischen Modells Leuchtkräfte, also entfernungsunabhängige Messgrößen errechnet. Für alle vier Spektralbereiche gilt, dass die VBLG höhere mittlere Leuchtkräfte bei einer breiteren Verteilung (d.h. bei höherer Standardabweichung) als die röntgenselektierten Galaxien zeigen. Dies wurde jeweils anhand der Häufigkeitsverteilung der Leuchtkräfte wie auch mit den statistischen Parametern demonstriert (siehe Abbildungen 4.2, 4.5, 4.8 und 4.11).

Die statistischen Parameter wurden nicht nur für die Teilsamples VBLG und XGAL ermittelt - letztere wurden zusätzlich noch in die Untergruppen ,röntgenselektierte AGN“ (XAGN) und „,öntgenselektierte Typ-1 AGN“ (XAGN-1) differenziert. Dabei zeigte sich, dass die Unterschiede in den mittleren Leuchtkräften der XGAL, XAGN und XAGN-1 in allen vier Spektralbereichen äußerst gering, d.h. weit unterhalb der jeweiligen Streuung sind (s. Tabellen 4.1, 4.2, 4.6 und 4.7).

Die drei Datensätze Röntgen, Radio und Ferninfrarot wurden unter dem Aspekt der jeweiligen Nachweisgrenze des entsprechenden Kataloges betrachtet. In allen Fällen liegt ein Großteil der Quellen nahe am Detektionslimit. Das trifft für die röntgenselektierten Galaxien in noch stärkerem Maße als für die VBLG zu. Der optische Datensatz wurde mit den Quellen des Veron-Kataloges (Véron-Cetty \& Véron 2001) verglichen - im Optischen liegen die Quellen unseres Samples im Bereich der helleren Veron-Galaxien.

Betrachtet man die Leuchtkräfte als Funktion der Rotverschiebung, findet man in allen vier Spektralbereichen eine enge Korrelation: die mittlere absolute Leuchtkraft steigt mit der Rotverschiebung stetig an. Dieser Effekt, der für flusslimitierte Sample unvermeidlich ist, wird als Malmquist-Bias bezeichnet (Gonzalez \& Faber 1997, Teerikorpi 1997) und in Abschnitt 4.7 ausführlich erläutert. Er läßt sich gut auch an den in den Graphiken eingezeichneten Detektionsgrenzen in Abhängigkeit von der Rotverschiebung erkennen und muss bei allen Interpretationen entsprechender Abbildungen stets berücksichtigt werden.

Das hier definierte Sample bzw. die beiden Teilsamples unterliegen neben dem Malmquist-Bias natürlich noch weiteren Selektionseffekten. Das ist nicht nur unvermeidlich, sondern in diesem Fall ausdrücklich erwünscht - die beiden Teilsamples wurden schließlich explizit aufgrund besonderer Eigenschaften selektiert. Dass es bislang generell keine AGN-Samples ohne Selektionseffekte gibt (,unbiased samples“), haben Urry \& Padovani (1995) überzeugend dargelegt. 


\subsubsection{Flussverhältnisse}

Eine besonders wirkungsvolle Methode, bestimmte Selektionseffekte und insbesondere auch entfernungsabhängige Einflüsse zu eliminieren, ist die Berechnung und Analyse von Flussverhältnissen. Fluss- bzw. Leuchtkraftverhältnisse sind prinzipiell entfernungsunabhängig, das heißt, sie sind auch davon unabhängig, ob das betrachtete Objekt absolut leuchtkräftig oder leuchtschwach ist.

Darüber hinaus bietet ein Flussverhältnis die Möglichkeit, die relativen Helligkeiten in zwei Wellenlängenbereichen gleichzeitig zu betrachten und mit weiteren Parametern zu korrelieren. Das in dieser Arbeit stets verwendete logarithmische Flussverhältnis entspricht bis auf einen Skalierungsfaktor dem in der Literatur ebenfalls gebräuchlichen Spektralindex.

Zunächst wurden die Flüsse und die korrespondierenden Leuchtkräfte aus jeweils zwei Frequenzbereichen miteinander verglichen und auf gegenseitige Abhängigkeiten untersucht. Anschließend wurde in Anlehnung an in der Literatur übliche Normierungen (Maccacaro et al. 1988, Helou et al. 1985, Keel 1993, Brinkmann et al. 1995) das entsprechende Flussverhältnis errechnet.

Die wichtigste Methode zur Untersuchung der Daten ist die Korrelationsanalyse. In dieser Arbeit wurden Rangkorrelationen berechnet, da diese keine zusätzlichen Annahmen über die Verteilungsfunktionen erfordern (s. Abschnitt 4.1). Aus einer Korrelation zweier Größen kann man schließen, dass es einen gemeinsamen physikalischen Ursprung bzw. eine kausale Verkettung dieser Größen gibt. Neben den Korrelationskoeffizienten wurden weiterhin jeweils die statistischen Parameter für die Teilsamples berechnet und die graphischen Auftragungen phänomenologisch bewertet.

\section{Gemeinsame Eigenschaften der Flussverhältnisse}

Sowohl aus den entsprechenden Graphiken wie auch quantitativ aus den Ergebnissen der Korrelationsanalyse ist unmittelbar ersichtlich, dass alle vier Leuchtkräfte untereinander korreliert sind. Wie ausführlich in Abschnitt 4.7 erläutert, ist dies jedoch keine physikalische Eigenschaft der Quellen, sondern beruht letztendlich auf den immer flusslimitierten Beobachtungsmöglichkeiten (s. auch Abbildung 4.14).

Physikalisch bedeutsamer sind Korrelationen der Flüsse. Hier konnte festgestellt werden, dass der Röntgenfluss mit keinem anderen Fluss korreliert ist, die Flüsse der anderen drei Spektralbereiche hingegen sehr wohl untereinander korreliert sind. Das legt den Schluss nahe, dass die Röntgenemission einerseits und die optische, Radio- und FIR-Emission andererseits unterschiedliche physikalische Ursachen haben oder aus unterschiedlichen Regionen stammen - erstere aus dem Aktiven Kern und damit aus einem 
sehr kleinen, zentralen Raumgebiet und letztere aus der ausgedehnten Starburstkomponente der Galaxie (s. Abschnitt 5.8.1).

Durch die Korrelationsanalyse der Flussverhältnisse mit den jeweils korrespondierenden Leuchtkräften zeigte sich, dass jedes Flussverhältnis mit jeweils einer dieser beiden Leuchtkräfte besonders stark korreliert, d.h. das Verhältnis wird jeweils von einem Frequenzband dominiert. Bei den Verhältnissen, in die die Radioleuchtkraft eingeht, ist jeweils diese dominant (s. Abschnitt 5.8.2).

Für alle sechs Flussverhältnisse konnten Hinweise auf eine Korrelation mit der Rotverschiebung festgestellt werden. Das könnte einerseits auf einem Entwicklungseffekt der Quellen (Osmer 2003), anderseits aber auch auf dem Zusammenspiel von Beobachtungslimit und in den jeweiligen Frequenzbändern unterschiedlichen Leuchtkraftfunktionen beruhen (s. Abschnitt 5.7). Für diese Arbeit ist allerdings noch interessanter, dass sich die Untergruppen VBLG und XAGN diesbezüglich nicht gleich verhalten: betrachtet man die Untergruppen einzeln, so korrelieren nicht mehr alle Flussverhältnisse mit der Rotverschiebung und es korrelieren ferner für VBLG und XAGN nicht die gleichen Verhältnisse. Dies ist ein starkes Indiz, dass es intrinsische Unterschiede zwischen VBLG und XAGN gibt.

\section{Folgerungen aus einzelnen Flussverhältnissen}

Da unser Sample aufgrund von optischen und Röntgeneigenschaften selektiert wurde, ist das entsprechende Flussverhältnis $f_{X / B}$ von besonderem Interesse. Es steht für nahe$\mathrm{zu}$ alle Quellen zur Verfügung.

Zunächst stellte sich heraus, dass die VBLG im Vergleich zu den XAGN nicht nur leuchtkräftiger im Optischen und im Röntgenbereich sind, sondern darüber hinaus auch ein höheres mittleres Flussverhältnis $f_{X / B}$ zeigen. In einem weiteren Schritt (s. Abschnitt 5.1) ließ sich dieses Ergebnis noch detaillierter formulieren, und wir konnten unsere Quellen in eine bereits bekannte, allgemeinere Sequenz von verschiedenen Sterntypen, normalen Galaxien und AGN (Maccacaro et al. 1988) einordnen und somit die hier zunächst rein formal eingeführte Typ-Sequenz BL Lac, VBLG, Seyfert-1, Seyfert1.5/1.8/1.9, Seyfert-2, LLAGN, LINER auch als Aktivitätssequenz interpretieren (vgl. Abbildung 5.3).

Für normale Galaxien ist eine Ferninfrarot-Radio-Korrelation bekannt und etabliert (z.B. de Jong et al. 1985, van Driel et al. 1991, Thean et al. 2001), die ursächlich darauf beruht, dass beide Spektralbereiche von Sternentstehungsprozessen dominiert werden (Heisler 1999). Unser Sample ist bestens geeignet, diese Beziehung für Aktive Galaxien zu untersuchen. Es zeigte sich, dass die XAGN trotz ihrer nuklearen Aktivität eindeutig der Ferninfrarot-Radio-Korrelation folgen und mit dem mittleren Flussverhältnis $\mathrm{f}_{\mathrm{FIR} / \mathrm{R}}$ für normale Galaxien (Helou et al. 1985) sehr gut vereinbar sind. Unter den VBLG sind 
drei Galaxien mit einem Radiofluss weit über dem aller anderen Quellen, weshalb die Vermutung nahe liegt, dass dies durch eine zusätzliche, bei den anderen Galaxien nicht vorhandene Radiokomponente verursacht ist. Ohne Berücksichtigung dieser drei Quellen korrelieren FIR- und Radiofluss auch für die VBLG, allerdings mit einem etwas niedrigeren mittleren $f_{F I R / R}$.

Das Verhältnis von Röntgen- und FIR-Fluss $f_{X / F I R}$ läßt einerseits unter Berücksichtigung aus der Literatur bekannter Obergrenzen für Starburst-Emission (Boller \& Bertoldi 1996, Boller 1999) den Schluss zu, dass für unsere Galaxien der Starburst-Beitrag zum Flussverhältnis $f_{X / F I R}$ vernachlässigbar ist. Andererseits ergab sich eine starke Korrelation von $\mathrm{f}_{\mathrm{X} / \mathrm{FIR}}$ mit der Röntgenleuchtkraft $\mathrm{L}_{\mathrm{X}}$. Beide Ergebnisse zusammen lassen sich so deuten, dass die Aktiven Kerne die Röntgenemission, die unterliegenden Galaxien hingegen die FIR-Emission dominieren.

Auf Sternentstehung in jüngerer Vergangenheit kann die Korrelation des Flussverhältnisses $\mathrm{f}_{\mathrm{FIR} / \mathrm{B}}$ mit der Ferninfrarot-Leuchtkraft LFIR zurückgeführt werden (Keel 1993, Klaas 1988, van Driel \& de Jong 1990). Neben der FIR- und Radio-Emission wird somit i.d.R. auch die optische B-Helligkeit unserer Quellen von Sternentstehung dominiert. Weiterhin zeigten sich bezüglich obiger Korrelation signifikante Differenzen zwischen XAGN und $\mathrm{VBLG}^{+}$(s. Abschnitt 5.4), die erneut auf intrinsische Unterschiede dieser beiden Untergruppen hindeuten.

Anhand des Flussverhältnisses $\mathrm{f}_{\mathrm{R} / \mathrm{B}}$ konnten nach Kellermann et al. (1989) sowie Falcke et al. (1996) radioleise und radiolaute Quellen unterschieden werden. Die VBLG sind mehrheitlich radiolaut (67\%), die XAGN hingegen nur zu 26\%, was mittelbar durch unsere Selektion bedingt ist. Ein Vergleich mit dem BQS-Sample von Kellermann et al. (1989) ergab, dass die XAGN bezüglich dieser Anteile „,normalen AGN“ entsprechen (s. Abschnitt 5.1).

Ebenso wie das Flussverhältnis $\mathrm{f}_{\mathrm{R} / \mathrm{B}}$ wird auch $\mathrm{f}_{\mathrm{X} / \mathrm{R}}$ von der Radioemission dominiert, was sich an einer starken Korrelation mit der Radioleuchtkraft $v L_{R}$ zeigt. Damit werden Ergebnisse von Brinkmann et al. (1995) sowie Reich et al. (2000) bestätigt (s. Abschnitt 5.6). Eine signifikante Abhängigkeit von AGN-Typ konnte für $f_{X / R}$ nicht festgestellt werden.

Betrachtet man die Ergebnisse der Multispektralanalyse gemeinsam, so lassen sie sich naheliegend dadurch erklären, dass optische, Radio- und FIR-Emission der großen Mehrheit der Quellen des Samples durch Sternentstehung dominiert werden, während hingegen der Starburst-Beitrag zur Röntgenemission vernachlässigbar und diese damit allein dem Aktiven Kern zuzuordnen ist. Signifikante Unterschiede zwischen XAGN und VBLG in den Korrelation von $\mathrm{f}_{\mathrm{FIR}} \times \mathrm{f}_{R}$ und $\mathrm{f}_{\mathrm{FIR} / \mathrm{B}} \times \mathrm{L}_{\mathrm{FIR}}$ deuten auf intrinsische physikalische Unterschiede dieser beiden Untergruppen hin. 


\subsection{Spektroskopie der Broad-Line Region}

Für alle Galaxien unseres Samples haben wir optische Spektren aufgenommen, die eine detaillierte Analyse der Objekte ermöglichten. Nach der genauen Klassifikation der Quellen war die Untersuchung der Broad-Line Region das zweite wesentliche Ziel der Spektroskopie - sie beschränkt sich naturgemäß auf die Typ-1 AGN des Samples. Da sie im Hinblick auf das „Unified Model“ von besonderer Wichtigkeit sind, wurden die Breite der erlaubten Emissionslinien und das Balmerdekrement genauer untersucht.

Wie bereits bei der Multifrequenzanalyse stützen sich die erzielten Ergebnisse auf die drei unabhängigen Methoden Korrelationsanalyse, statistische Analyse, sowie graphische Darstellung und Bewertung, die im Wesentlichen zu gleichen Resultaten führten und damit die Signifikanz der getroffenen Aussagen entscheidend erhöhen.

\subsubsection{Die Emissionslinienbreite}

Die Breite der erlaubten Emissionslinien dient nicht nur zur Unterscheidung von Typ-1 und Typ-2 AGN, sie ist auch ausschlaggebend zur weiteren Differenzierung der Typ-1 AGN in NLS1, Seyfert-1 und BLRG/VBLG. Die Übergänge zwischen den letztgenannten Typ-1 AGN sind hinsichtlich der Linienbreiten fließend, die Erklärung der physikalischen Zusammenhänge sind Gegenstand des „Unified Models“.

Gemäß der Gleichung 6.1 ist die Geschwindigkeitsdispersion in der Broad-Line Region und damit auch die intrinsische Linienbreite direkt mit dem Gravitationspotenzial des AGN bzw. der Zentralmasse verknüpft. In die beobachtete Linienbreite geht allerdings ferner die Geometrie der BLR ein, die noch nicht endgültig geklärt ist.

Weitgehend unstrittig ist, dass sich im Zentrum eines AGN eine Akkretionsscheibe befindet. Wie in Abschnitt 1.3.5 dargestellt, haben wir für die Seyfert-1 Galaxie Mrk 110 starke Evidenz dafür gefunden, dass auch die BLR scheibenförmig ist und aus Wolken besteht, die in einem von der Akkretionsscheibe abströmenden Wind mitbewegt werden (Kollatschny \& Bischoff 2002).

Während im einfachsten Fall einer sphärisch-symmetrischen BLR der Blickwinkel keinen Einfluss auf die beobachtete Linienbreite hätte, wäre er bei einer scheibenförmigen BLR letztlich ausschlaggebend (Welsh \& Horne 1991, Perez et al. 1992a,b, Wills \& Brotherton 1995).

Wir haben für alle Galaxien mit breiten Linien bzw. breiten Linienkomponenten durch einen Mehrkomponentenfit an die Balmerlinien $\mathrm{H} \alpha$ und/oder $\mathrm{H} \beta$ die entsprechenden Halbwertsbreiten FWHM bestimmt. Für die Galaxien, für die wir beide Balmerlinien messen konnten, haben wir im Mittel bei $\mathrm{H} \beta$ eine etwas höhere Linienhalbwertsbreite bei als bei $\mathrm{H} \alpha$ festgestellt. Dies ist konform mit anderen Beobachtungen (Bischoff, 
Kollatschny 1999, Kollatschny, Bischoff \& Dietrich 2000) sowie auch mit Photoionisationsmodellen (Collin-Souffrin \& Dumont 1989, Rokaki et al. 1992).

Unser Sample hat den entscheidenden Vorteil, dass es Typ-1 AGN mit einem durchgehenden Spektrum von Linienbreiten von $1000 \mathrm{~km} \mathrm{~s}^{-1}$ bis $16000 \mathrm{~km} \mathrm{~s}^{-1}$ FWHM umfasst (s. Abbildung 6.5). Diesen großen Bereich haben wir in vier Intervalle unterteilt (s. Abschnitt 6.2), um anhand dieser Intervalle Abhängigkeiten von der Linienbreite statistisch analysieren zu können. Weiterhin haben wir die Linienbreite auf Korrelationen mit den Leuchtkräften und den Flussverhältnissen untersucht.

\subsubsection{Zusammenhänge mit anderen Parametern}

Es zeigte sich eine starke Korrelation der Linienbreite mit der Radioleuchtkraft und eine weniger starke, aber signifikante Korrelation mit der absoluten optischen Helligkeit. Ferner ergaben sich Korrelationen mit genau den drei Flussverhältnissen, in die der Radiofluss eingeht, während die anderen drei Flussverhältnisse nicht mit der Linienbreite korrelieren.

Noch interessanter als die Korrelationen sind die Ergebnisse bzgl. des wichtigen Flussverhältnisses $\mathrm{f}_{\mathrm{X} / \mathrm{B}}$, das auch als Maß für die nichtthermische Aktivität angesehen werden kann: hier zeigen sich deutliche Diskrepanzen zwischen XAGN und VBLG, die nicht allein auf einen Effekt unterschiedlicher Linienbreitenverteilung in den beiden Untergruppen zurückgeführt werden können. Dies wurde in Abschnitt 6.2.2 diskutiert.

Wie in Abbildung 6.10 zu erkennen ist, konnte eine Grenzlinie so in das Diagramm eingefügt werden, dass sich die XAGN-1 nahezu symmetrisch um diese Linie verteilen, die VBLG aber ausnahmlos darüber liegen und es sich somit um eine Grenze zwischen den durch VBLG besetzten und unbesetzten Bereichen des Diagramms handelt. Das heißt, bei den VBLG fehlen Objekte mit niedriger Halbwertsbreite und geringem $f_{X / B}$ völlig, während sich in diesem Bereich des Diagramms sehr wohl etliche XAGN-1 befinden. Die ebenfalls in Abbildung 6.10 eingezeichneten Mittelwerte für die vier Halbwertsbreitenintervalle unterscheiden sich nur marginal, was darauf hindeutet, dass es sich hierbei nicht primär um einen Effekt der Linienbreite handelt.

Dieses Ergebnis ist ein weiterer, sehr deutlicher Hinweis auf intrinsische physikalische Unterschiede zwischen XAGN und VBLG. Es lässt ferner Rückschlüsse auf Geometrie und Inklination der Broad-Line Region zu und liefert somit Einschränkungen für die Modellvorstellungen von AGN. Es ist konform mit vergleichbaren Ergebnissen von Wills \& Browne (1986), Wills \& Brotherton (1995) und Brotherton (1996), die im folgenden Abschnitt 7.3.3 noch genauer diskutiert werden.

Im Rahmen des „Unified Models“ läßt sich ein hohes Verhältnis $\mathrm{f}_{\mathrm{X} / \mathrm{B}}$ mit einem freien, nicht absorbierten Sehstrahl auf den Aktiven Kern assoziieren, da die Röntgenstrahlung 
aus dem unmittelbaren Kernbereich kommt, während zur optischen Emission größere Raumgebiete beitragen. Dies ist die sog. „face-on“ Orientierung und entspricht einer geringen Inklination. Ein kleineres Verhältnis $\mathrm{f}_{\mathrm{X} / \mathrm{B}}$ deutet dementsprechend auf einen eingeschränkteren Blick auf die zentrale Quelle hin und damit auf eine größere Inklination.

Ein Zusammenhang zwischen $\mathrm{f}_{\mathrm{X} / \mathrm{B}}$ bzw. Inklination und Linienbreite, wie wir ihn für die VBLG beobachten, läßt sich nicht mit einer sphärisch symmetrischen Broad-Line Region erklären (bei der die Linienbreite unabhängig vom Inklinationswinkel wäre), sehr wohl aber mit einer scheibenförmigen BLR.

\subsubsection{Das Scheiben-Wind-Modell der BLR}

Mit Beobachtungen aus einer mehrwöchigen Variabilitätskampagne zur Seyfert-1 Galaxie Mrk 110 haben wir starke Evidenz für eine scheibenförmige Broad-Line Region in dieser Galaxie gefunden (Kollatschny et al. 2001, Kollatschny \& Bischoff 2002).

Diese Beobachtungen entsprechen nahezu perfekt dem Scheiben-Wind-Modell von Königl \& Kartje (1994) und Kartje et al. (1997), in dem die BLR von Gaswolken gebildet wird, die in einem von der Akkretionsscheibe abströmenden Wind nach außen transportiert werden. Die äußeren, kühlen Bereiche der Akkretionsscheibe bilden den abschattenden Torus, der eine wesentliche Komponente des „Unified Models“ darstellt, und es ist somit keine zusätzliche, hypothetische Komponente notwendig. Die Dicke der kühlen Scheibe entspricht den Anforderungen des „Unified Models“.

Das Scheiben-Wind-Modell ist eine detaillierte Vorstellung über die Broad-Line Region und kann als Konkretisierung des allgemeineren „Unified Models“ verstanden werden. Es ist in Abbildung 7.1 schematisch dargestellt, wobei es auf die für Mrk 110 bestimmten Parameter (Masse des zentralen Schwarzen Lochs: $1.4 \pm 0.3 \cdot 10^{8} \mathrm{M}_{\odot}$, Kollatschny 2003) adaptiert ist. Die eingezeichnete Größenskala haben wir durch die Beobachtung verschiedener Emissionslinien (Balmerlinien, He I und He II) ausgemessen.

Da sich die scheibenförmige BLR in der Ebene der Akkretionsscheibe befindet, deren äußere Bereiche das Zentrum für Beobachter in derselben Ebene verdecken, hätte ein flacher Blickwinkel bzw. eine große Inklination zur Folge, dass zum einen der Sehstrahl teilabsorbiert wäre, zum anderen in jedem Fall die Radialkomponente der Scheibenrotation zu einer großen Linienbreite der BLR-Emission führen würde - wie für die VBLG beobachtet gäbe es keine kleinen Linienbreiten in Verbindung mit einem teilabsorbierten $\mathrm{f}_{\mathrm{X} / \mathrm{B}}$.

Für einen steilen Blickwinkel entsprechend einer geringen Inklination läge eine freie Sicht auf das Zentrum und somit keine Absorption vor, die Radialkomponente der Rotation wäre aber gering. Stattdessen hätte der von der Scheibe abströmende Wind, der die 


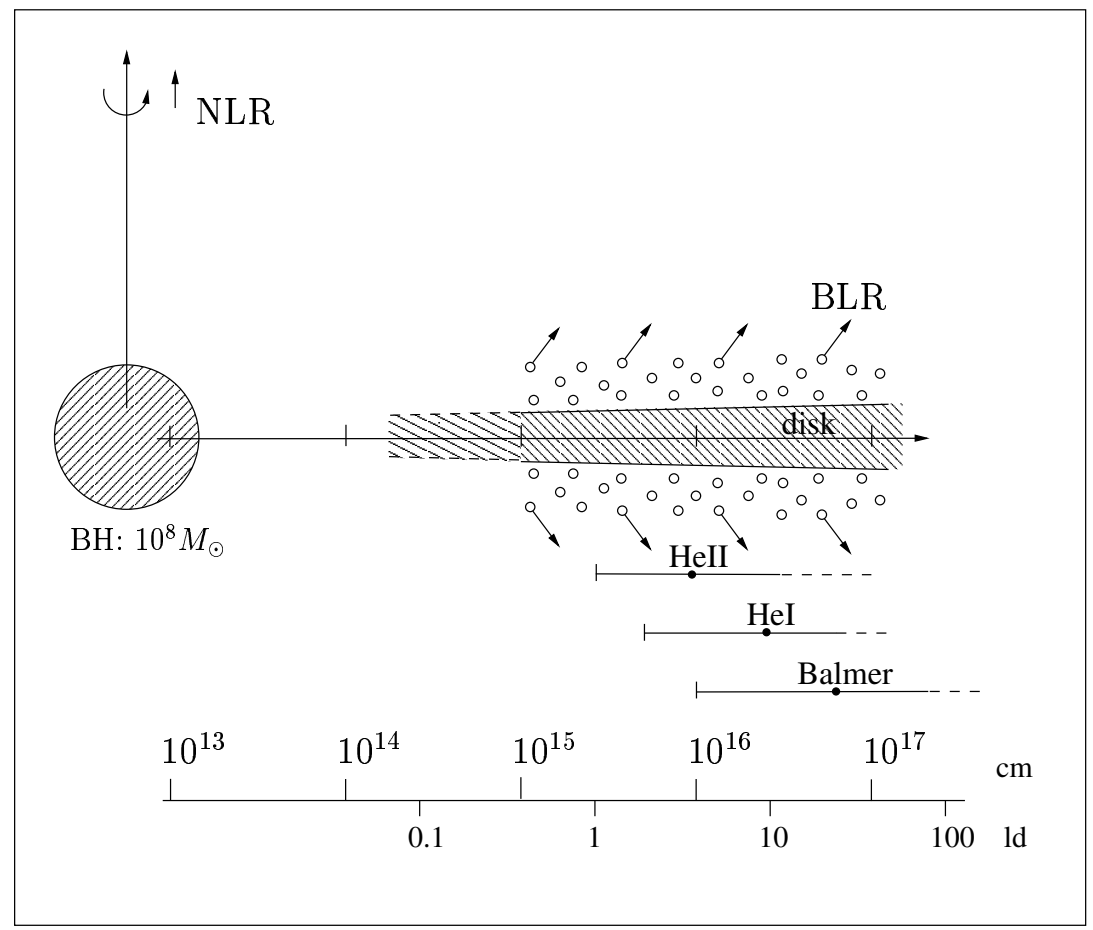

\begin{abstract}
Abbildung 7.1: Modell des Zentralbereichs der Seyfert-1 Galaxie Mrk 110 (Kollatschny \& Bischoff 2002). Die Broad-Line Region hat eine scheibenförmige Geometrie und besteht aus Gaswolken, die im von der Akkretionsscheibe abströmenden Wind mitgetrieben werden (durch Pfeile symbolisiert). Die äußeren, kühlen Bereiche der Akkretionsscheibe bilden einen abschattenden Torus um das Zentrum. Die Raumgebiete der verschiedenen Emissionen (Balmer, He I, He II) konnten genau ausgemessen werden, die logarithmische Größenskala ist sowohl in $\mathrm{cm}$ als auch in Lichttagen (ld) angegeben.
\end{abstract}

BLR-Wolken transportiert, eine große Radialkomponente und bestimmte die beobachtete Linienbreite. Über die Geschwindigkeit des Windes machen Königl \& Kartje (1994) und Kartje et al. (1997) keine quantitativen Angaben, sie geben aber qualitativ an, dass die Trajektorien des Windes und damit auch der BLR-Wolken vorwiegend vertikal in Bezug auf die Scheibe verlaufen.

Trifft dieses Modell für die VBLG zu, so könnte aus dem beobachteten Linienbreitenbereich von etwa $2000 \mathrm{~km} \mathrm{~s}^{-1}$ bis $16000 \mathrm{~km} \mathrm{~s}^{-1}$ geschlossen werden, dass auch die Windgeschwindigkeiten in den verschiedenen Galaxien zumindest diesen Bereich überdecken.

Photoionisationsrechnungen zu einem Scheiben-Wind-Modell der BLR haben Chiang \& Murray (1996) sowie Murray \& Chiang $(1997,1998)$ durchgeführt. Sie haben gezeigt, dass aufgrund des von der Scheibe abströmenden Windes die breiten Emissionslinien- 
profile auch für eine scheibenförmige BLR eine normale Form mit zentralem Maximum haben können, obwohl ansonsten sog. ,double peaked“ DP-Profile für ScheibenGeometrien typisch sind (s. Abschnitt 2.1). Für ihr Modell geben sie an, dass die Scheibenrotation gegenüber der Geschwindigkeit des Windes dominiert.

Eine noch frühere Beschreibung eines Scheiben-Wind-Modells geht auf Blandford \& Payne (1982) zurück und wurde von Emmering et al. (1993) konkretisiert. In diesem Modell werden die BLR-Wolken durch Strahlungdruck von der Akkretionsscheibe weg beschleunigt und durch die Zentrifugalkräfte entlang der Magnetfeldlinien nach außen geschleudert. Dabei erreichen sie ein Vielfaches der ursprünglichen Keplergeschwindigkeiten. Neuere Rechnungen zur Akkretionsrate in diesem Modell wurden von Blandford \& Begelman (1999) publiziert. Insbesondere kommen sie zu dem Schluss, dass nur ein kleiner Teil der einfallenden Materie tatsächlich vom Schwarzen Loch akkretiert wird, der Rest hingegen als Wind wieder nach außen strömt.

Dass das Scheiben-Wind-Modell hervorragend die Zweiteilung des $\mathrm{f}_{\mathrm{X} / \mathrm{B}}$-FWHM- Diagramms (Abbildung 6.10) für die VBLG erklärt, diese Zweiteilung aber nicht für die XAGN beobachtet wird, schließt das Modell für letztere nicht zwangsläufig aus. Der Unterschied könnte in einzelnen Parametern begründet sein - wenn z.B. für die XAGN die Windgeschwindigkeit gegenüber der Rotationsgeschwindigkeit der Scheibe dominierte (wie von Emmering et al. (1993) postuliert), sollte man keine Zweiteilung erwarten. Es zeigt aber in jedem Fall, dass es irgendwie geartete intrinsische Unterschiede zwischen XAGN und VBLG gibt.

Eine dem hier gefundenen $\mathrm{f}_{\mathrm{X} / \mathrm{B}}$-FWHM-Zusammenhang vergleichbare Beobachtung haben Wills \& Browne (1986) gemacht, indem sie für ein Sample Aktiver Galaxien das Verhältnis R von Kernradiofluss zu ausgedehntem Radiofluss und die H $\beta$ Halbwertsbreite untersuchten.

Das Verhältnis R interpretierten sie als Maß für den Blickwinkel, was auch durch spätere Messungen von Ghisellini et al. (1993) bestätigt wurde: schaut man „face-on“ auf den AGN, so sieht man eine hohe Kernhelligkeit im Radiobereich bei geringem ausgedehnten Radiofluss und findet damit ein großes R. Entsprechend ist R bei einer ,edge-on“ Orientierung klein. Diese Interpretation geht auf das „Doppler-Boosting-Modell“ (z.B. Blandford \& Rees 1978, Orr \& Browne 1982, Hough \& Readhead 1989) zurück, das in Abschnitt 1.3.2 beschrieben wurde.

Aus dem Zusammenhang von R mit der H $\beta$-Halbwertsbreite folgerten Wills \& Browne (1986), dass sich das linienemittierende Gas überwiegend in einer senkrecht zur Radioachse orientierten Scheibe befindet.

In neueren Arbeiten fanden Wills \& Brotherton (1995) sowie Brotherton (1996) für das Sample von 1986 wie auch für 41 weitere radiolaute AGN, dass das Verhältnis von Kernradiohelligkeit zur optischen V-Helligkeit $\mathrm{R}_{V}$ ein besseres Maß als $\mathrm{R}$ für die Orientierung des AGN ist. Entsprechend fanden sie eine stärkere Korrelation zwischen 
$\mathrm{R}_{V}$ und $\mathrm{FWHM}(\mathrm{H} \beta)$ als zwischen R und $\mathrm{FWHM}(\mathrm{H} \beta)$, was die prinzipiellen Ergebnisse der früheren Arbeit nicht nur bestätigt, sondern untermauert.

Die Beobachtungen von Wills et al. sind konsistent mit den Ergebnissen der vorliegenden Arbeit und liefern weitere Evidenz für eine scheibenförmige Broad-Line Region.

\subsubsection{Das Balmerdekrement}

Auch das Balmerdekrement $\mathrm{H} \alpha / \mathrm{H} \beta$ wurde auf Korrelationen mit den Multifrequenzdaten untersucht. Es ergab sich einerseits aufgrund der numerischen Analyse nur für die Radioleuchtkraft und dementsprechend auch für die drei Flussverhältnisse, in die der Radiofluss eingeht, eine mäßig starke Korrelation mit dem Balmerdekrement. Die korrespondierenden graphischen Auftragungen stellen andererseits die Signifikanz dieser Korrelationen in Frage. Es konnte kein signifikanter Unterschied zwischen den Untergruppen XAGN und VBLG festgestellt werden.

Die Analyse von Balmerdekrement und Linienhalbwertsbreite hat keinen Zusammenhang dieser beiden Größen ergeben. Auch hierbei ist wiederum kein signifikanter Unterschied zwischen VBLG und XAGN feststellbar. Das ist ein wichtiges Ergebnis, weil im Rahmen des „Unified Models“ BLRG bzw. VBLG häufiger ein hohes Balmerdekrement zugeschrieben wurde (z.B. Wills 1999, siehe auch Abschnitt 2.1). Dabei wurde ein gegenüber dem Normalwert der Photoionisationstheorie (Osterbrock 1989) erhöhtes Balmerdekrement auf partielle Absorption durch den Staubtorus und somit auf eine hohe Inklination des AGN zurückgeführt (s. Abbildung 1.1). Diese Interpretation kann durch unser Sample nicht bestätigt werden.

\subsection{Schlussfolgerungen für das „Unified Model“}

Diese Arbeit hat gezeigt, dass die gleichzeitige Analyse möglichst vieler Parameter Aktiver Galaxien essentiell ist, um Unterschiede bzw. Gemeinsamkeiten verschiedener AGN-Typen zu erkennen und die Modellvorstellungen damit zu konfrontieren. Die parallele Untersuchung von multispektralen Kontinuumsdaten und optischen Spektren, die es bisher für kein Sample vergleichbarer Größe gab, hat sich somit als sehr erfolgreich erwiesen.

\section{Sind XAGN normale AGN?}

Vor der genauen Analyse der röntgenselektierten XAGN stellte sich die Frage, ob diese aufgrund besonderer Eigenschaften zuvor unentdeckt geblieben waren und somit eine 
eigene Klasse Aktiver Galaxien darstellen würden. Die ausführlich untersuchten Multifrequenzdaten inklusive der optischen Spektren lieferten keinerlei Hinweise, dass die XAGN außergewöhnliche AGN sind. Die Schlussfolgerung kann nur sein, dass wir zum einen durchschnittliche AGN selektiert haben, die zuvor rein statistisch unentdeckt geblieben waren, und dass zum anderen unser Selektionsverfahren hocheffizient im Auffinden solcher AGN ist.

Der hohen Anteil von Übergangstypen Sy1.5/1.8/1.9 sowie Sy2/LINER/LLAGN unter den XAGN ist mit einfachen Vorstellung des „Unified Models“ nicht vereinbar, da bei diesen die Röntgenstrahlung teilweise oder ganz absorbiert wäre. Die Erweiterung um eine zusätzliche, z.B. am inneren Torus gestreute Röntgenkomponente könnte hier die Lösung sein.

\section{Unterschiede zwischen XAGN und VBLG}

Im direkten Vergleich von XAGN und VBLG haben sich an mehreren Stellen Hinweise ergeben, dass es intrinsische physikalische Unterschiede zwischen diesen Typen gibt: die Korrelationen der Flussverhältnisse mit der Rotverschiebung sind für die beiden Untergruppen deutlich unterschiedlich; sowohl bzgl. der FIR-R-Korrelation als auch $\operatorname{der} \mathrm{f}_{\mathrm{FIR} / \mathrm{B}}-\mathrm{L}_{\mathrm{FIR}}$-Korrelation zeigen sich signifikante Unterschiede, und insbesondere im FWHM- $f_{X / B}$-Diagramm unterscheiden sich XAGN und VBLG stark voneinander.

Hier können keinesfalls Orientierungeffekte allein die Unterschiede erklären. Auch die An- oder Abwesenheit einzelner Komponenten im „Unified Model“ scheint dafür nicht ausreichend. Diese Beobachtungen sind wichtige Einschränkungen, die ein universelles „Unified Model“ künftig berücksichtigen müsste.

\section{Struktur der Broad-Line Region}

Ein fundamentaler Parameter für Typ-1 AGN ist die Breite der erlaubten Emissionslinien. Unser Sample eignet sich besonders, die Broad-Line Region anhand der Linienbreite zu parametrisieren, weil es das gesamte Geschwindigkeitsspektrum von $1000 \mathrm{~km} \mathrm{~s}^{-1}$ bis $16000 \mathrm{~km} \mathrm{~s}^{-1}$ kontinuierlich überdeckt und sich nicht wie viele andere Samples auf distinkte Typen beschränkt.

Zumindest für die VBLG haben diese Untersuchungen Evidenz für ein Scheiben-WindModell der BLR ergeben, von denen verschiedene Varianten diskutiert wurden. Dies ist auch deswegen von großer Bedeutung, weil es mit anderen aktuellen Beobachtungen einhergeht und klassische, weitverbreitete Vorstellung über die BLR deutlich erweitert sowie das „Unified Model“ in einem wichtigen Punkt entscheidend konkretisiert. 


\subsection{Ausblick}

Sowohl Qualität als auch Quantität von Datensätzen und Archiven, wie sie hier benutzt wurden, machen gegenwärtig große Fortschritte. Ebenso steigen Verfügbarkeit und Beobachtbarkeit optischer Spektren schnell an. Diese Untersuchungen und die Signifikanz der Ergebnisse werden sich durch den Einsatz neuer Daten künftig noch deutlich verbessern lassen.

Wie in Kapitel 1 beschrieben, gibt es etliche neue Beobachtungsbefunde, die bisherige Modellvorstellungen Aktiver Galaxien teilweise unterstützen, teilweise aber mit ihnen nicht konform sind. Auch die Ergebnisse dieser Arbeit stellen konkrete und substanzielle neue Randbedingungen an die Theorie Aktiver Galaxien und werden bei der Weiterentwicklung der Modelle berücksichtigt werden müssen. 


\section{Kapitel 8}

\section{Anhang}

Tabelle 8.1: Multifrequenzdaten des Samples gemäß den Ausführungen in Kapitel 4. Neben den Flüssen und Leuchtkräften in den vier untersuchten Frequenzbändern B, X, R und FIR ist noch

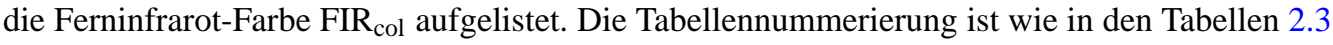
und 3.1 eingeführt.

\begin{tabular}{rccccccccc}
\hline \hline $\mathrm{Nr}$. & $\mathrm{m}_{\mathrm{B}}$ & $\mathrm{M}_{\mathrm{B}}$ & $\log \mathrm{f}_{\mathrm{X}}$ & $\log \mathrm{L}_{\mathrm{X}}$ & $\log \mathrm{f}_{R}$ & $\log \mathrm{v} \mathrm{L}_{\mathrm{R}}$ & $\log \mathrm{f}_{\mathrm{FIR}}$ & $\log \mathrm{L}_{\mathrm{FIR}}$ & $\mathrm{FIR}_{\mathrm{col}}$ \\
\hline 1 & 15.66 & -20.21 & -11.75 & 42.68 & -24.99 & 38.58 & & & \\
2 & 17.55 & -19.34 & & & -24.15 & 39.83 & & & \\
3 & 14.10 & -26.41 & -10.44 & 45.84 & -24.03 & 41.40 & -10.17 & 46.11 & -0.24 \\
4 & 14.36 & -22.85 & -10.45 & 44.51 & -24.78 & 39.33 & -10.76 & 44.20 & -0.27 \\
5 & 18.70 & -21.88 & -11.41 & 44.90 & -22.68 & 42.78 & & & \\
6 & 17.30 & -20.43 & -12.20 & 42.97 & -22.51 & 41.81 & & & \\
7 & 16.69 & -23.55 & -10.97 & 45.20 & -22.81 & 42.51 & & & \\
8 & 15.50 & -21.39 & -10.17 & 44.67 & -22.47 & 41.51 & & & \\
9 & 14.37 & -22.45 & -10.79 & 44.01 & -21.89 & 42.06 & -10.84 & 43.96 & -0.48 \\
10 & 17.00 & -19.82 & -12.18 & 42.63 & -22.68 & 41.27 & -10.64 & 44.17 & -0.53 \\
11 & 15.77 & -24.93 & & & -22.84 & 42.66 & & & \\
12 & 16.00 & -22.25 & -11.16 & 44.22 & -22.79 & 41.73 & & & \\
13 & 16.00 & -25.53 & -11.41 & 45.28 & -22.87 & 42.97 & & & \\
14 & 15.47 & -25.05 & -11.08 & 45.20 & -22.54 & 42.89 & & & \\
15 & 16.10 & -20.15 & -10.85 & 43.72 & -23.44 & 40.29 & -9.36 & 45.21 & -1.62 \\
16 & 15.12 & -19.89 & -11.72 & 42.37 & -23.84 & 39.39 & & & \\
17 & 14.32 & -21.54 & & & & & -10.43 & 43.99 & -0.41 \\
18 & 15.00 & -21.62 & -10.49 & 44.24 & -25.37 & 38.50 & & & \\
19 & 15.08 & -22.09 & -12.31 & 42.64 & & & & & \\
20 & 17.00 & -24.59 & & & & & & & \\
21 & 15.23 & -23.40 & -10.53 & 45.00 & -25.36 & 39.32 & -10.53 & 45.00 & -0.13 \\
22 & 14.50 & -21.94 & -10.23 & 44.43 & -24.46 & 39.34 & & & \\
\hline \hline
\end{tabular}

$\mathrm{m}_{\mathrm{B}}, \mathrm{M}_{\mathrm{B}}$ in mag.

$\mathrm{f}_{\mathrm{X}}, \mathrm{f}_{\mathrm{FIR}}$ in erg s $\mathrm{cm}^{-1} ; \mathrm{f}_{R}$ in erg s $\mathrm{s}^{-1} \mathrm{~cm}^{-2} \mathrm{~Hz}^{-1}$

$\mathrm{L}_{\mathrm{X}}, v \mathrm{~L}_{\mathrm{R}}, \mathrm{L}_{\mathrm{FIR}}$ in erg s ${ }^{-1}$ 


\begin{tabular}{|c|c|c|c|c|c|c|c|c|c|}
\hline Nr. & $\mathrm{m}_{\mathrm{B}}$ & $\mathrm{M}_{\mathrm{B}}$ & $\log f_{X}$ & $\log \mathrm{L}_{X}$ & $\log \mathrm{f}_{R}$ & $\log v L_{R}$ & $\log f_{\mathrm{FIR}}$ & $\log L_{F I R}$ & $\mathrm{FIR}_{\mathrm{col}}$ \\
\hline 23 & 14.76 & -21.73 & & & -24.92 & 38.90 & -10.41 & 44.27 & -0.12 \\
\hline 24 & 12.50 & -20.28 & -11.32 & 41.87 & -24.49 & 37.85 & -10.07 & 43.12 & -0.11 \\
\hline 25 & 15.40 & -22.40 & -10.78 & 44.42 & -24.01 & 40.34 & & & \\
\hline 26 & 15.41 & -23.43 & -10.79 & 44.83 & -25.13 & 39.63 & & & \\
\hline 27 & 16.06 & -22.37 & -10.92 & 44.53 & & & & & \\
\hline 28 & 14.99 & -22.81 & -11.94 & 43.26 & & & & & \\
\hline 29 & 15.43 & -25.02 & & & -24.66 & 40.75 & -10.61 & 45.65 & -0.40 \\
\hline 30 & 16.80 & -24.70 & & & -23.17 & 42.66 & & & \\
\hline 31 & 18.10 & -23.23 & -11.24 & 45.37 & -22.63 & 43.13 & & & \\
\hline 32 & 16.38 & -26.02 & -11.21 & 45.82 & -22.56 & 43.62 & & & \\
\hline 33 & 17.00 & -24.24 & -11.06 & 45.51 & -23.08 & 42.64 & & & \\
\hline 34 & 18.00 & -22.69 & -11.21 & 45.15 & -24.28 & 41.22 & & & \\
\hline 35 & 14.82 & -27.22 & -10.84 & 46.05 & -22.53 & 43.51 & & & \\
\hline 36 & 15.46 & -26.26 & -11.12 & 45.64 & -22.75 & 43.16 & & & \\
\hline 37 & 15.91 & -23.70 & -10.80 & 45.12 & -22.62 & 42.45 & & & \\
\hline 38 & 15.50 & -24.25 & -11.65 & 44.33 & -23.19 & 41.94 & & & \\
\hline 39 & 15.33 & -24.03 & -10.69 & 45.13 & -22.80 & 42.17 & -10.83 & 44.99 & -0.36 \\
\hline 40 & 17.00 & -20.33 & -11.62 & 43.39 & -25.26 & 38.90 & & & \\
\hline 41 & 15.68 & -25.31 & -11.94 & 44.53 & -23.97 & 41.65 & & & \\
\hline 42 & 15.41 & -23.25 & -10.84 & 44.71 & -24.74 & 39.95 & -10.94 & 44.60 & -0.33 \\
\hline 43 & 14.55 & -20.46 & -9.88 & 44.20 & -24.54 & 38.69 & -10.38 & 43.70 & -0.14 \\
\hline 44 & 16.42 & -22.89 & -11.34 & 44.46 & -24.87 & 40.08 & & & \\
\hline 45 & & & -11.30 & & & & & & \\
\hline 46 & 16.10 & & -11.62 & & -25.26 & & & & \\
\hline 47 & 15.40 & & -11.44 & & -23.89 & & & & \\
\hline 48 & 21.10 & & -11.70 & & -25.34 & & & & \\
\hline 49 & 17.90 & -24.53 & -12.47 & 45.00 & & & & & \\
\hline 50 & 15.82 & -19.63 & -11.71 & 42.55 & -25.29 & 38.11 & -10.64 & 43.62 & -0.43 \\
\hline 51 & 15.70 & -20.71 & -11.63 & 43.01 & & & & & \\
\hline 52 & 15.60 & -20.38 & -11.44 & 43.03 & -24.90 & 38.71 & -10.42 & 44.05 & -0.33 \\
\hline 53 & 15.70 & -20.60 & -11.31 & 43.64 & & & & & \\
\hline 54 & 16.00 & -19.17 & -12.19 & 42.32 & & & & & \\
\hline 55 & 17.46 & -20.23 & -11.09 & 44.07 & & & -10.64 & 44.52 & -0.49 \\
\hline 56 & 14.60 & -20.47 & -11.07 & 43.40 & & & -10.11 & 44.00 & -0.21 \\
\hline 57 & 14.20 & -22.76 & -11.13 & 44.10 & & & -10.32 & 44.54 & -0.28 \\
\hline 58 & 15.30 & -20.77 & -10.68 & 44.19 & & & -10.52 & 43.98 & -0.91 \\
\hline 59 & 14.60 & -20.28 & -11.26 & 43.12 & & & -10.46 & 43.57 & -0.41 \\
\hline 60 & 15.85 & -19.08 & -11.26 & 42.79 & & & & & \\
\hline 61 & 14.90 & -22.00 & -11.64 & 43.56 & -23.97 & 40.55 & & & \\
\hline 62 & 14.10 & -20.90 & -11.19 & 43.24 & -24.41 & 38.81 & -10.07 & 44.01 & -0.12 \\
\hline 63 & 13.30 & -21.48 & -10.38 & 43.61 & -24.87 & 38.26 & -10.43 & 43.56 & -0.58 \\
\hline 64 & 12.80 & -20.90 & -11.28 & 42.63 & & & -10.42 & 43.14 & -0.39 \\
\hline 65 & 16.30 & -19.72 & -11.37 & 43.47 & & & & & \\
\hline 66 & 14.30 & -21.32 & -11.89 & 42.79 & -25.14 & 38.33 & & & \\
\hline
\end{tabular}

$\mathrm{m}_{\mathrm{B}}, \mathrm{M}_{\mathrm{B}}$ in mag.

$\mathrm{f}_{\mathrm{X}}, \mathrm{f}_{\mathrm{FIR}}$ in erg s${ }^{-1} \mathrm{~cm}^{-2} ; \mathrm{f}_{R}$ in erg s $\mathrm{s}^{-1} \mathrm{~cm}^{-2} \mathrm{~Hz}^{-1}$

$\mathrm{L}_{\mathrm{X}}, v \mathrm{~L}_{\mathrm{R}}, \mathrm{L}_{\mathrm{FIR}}$ in erg $\mathrm{s}^{-1}$ 


\begin{tabular}{|c|c|c|c|c|c|c|c|c|c|}
\hline Nr. & $\mathrm{m}_{\mathrm{B}}$ & $\mathrm{M}_{\mathrm{B}}$ & $\log f_{X}$ & $\log L_{X}$ & $\log \mathrm{f}_{R}$ & $\log v L_{R}$ & $\log f_{\mathrm{FIR}}$ & $\log L_{F I R}$ & $\mathrm{FIR}_{\mathrm{col}}$ \\
\hline 67 & 15.71 & -21.68 & -11.31 & 43.73 & & & & & \\
\hline 68 & 13.70 & -21.43 & -11.34 & 43.14 & -24.36 & 38.91 & -9.64 & 44.49 & -0.34 \\
\hline 69 & 14.80 & -20.79 & -11.62 & 43.05 & & & & & \\
\hline 70 & 13.30 & -21.13 & -11.24 & 42.61 & -25.47 & 37.53 & -10.52 & 43.33 & -0.62 \\
\hline 71 & 16.50 & -20.78 & -11.43 & 43.56 & & & & & \\
\hline 72 & 16.00 & -19.92 & -10.76 & 43.69 & & & & & \\
\hline 73 & 14.60 & -19.42 & -11.14 & 42.90 & -25.09 & 37.74 & & & \\
\hline 74 & 15.20 & -20.60 & -11.67 & 43.08 & -24.97 & 38.57 & -10.38 & 44.02 & -0.43 \\
\hline 75 & 15.50 & -19.79 & -10.92 & 43.64 & -25.41 & 37.93 & -10.85 & 43.35 & -0.79 \\
\hline 76 & 15.70 & -21.75 & -11.46 & 43.96 & & & & & \\
\hline 77 & 18.00 & -20.75 & -11.50 & 44.08 & & & & & \\
\hline 78 & 15.30 & -20.77 & -11.28 & 43.22 & -25.49 & 38.16 & & & \\
\hline 79 & 13.90 & -21.41 & -11.20 & 43.35 & -22.46 & 41.43 & & & \\
\hline 80 & 15.10 & -20.55 & -11.82 & 42.88 & & & & & \\
\hline 81 & 14.80 & -23.82 & -11.05 & 44.84 & -23.34 & 41.87 & & & \\
\hline 82 & 14.60 & -21.51 & -11.33 & 43.55 & & & & & \\
\hline 83 & 15.10 & -18.55 & -11.72 & 41.81 & -25.31 & 37.37 & & & \\
\hline 84 & 15.70 & -20.52 & -11.53 & 43.04 & -25.15 & 38.56 & & & \\
\hline 85 & 16.74 & -21.12 & -11.12 & 44.10 & -24.89 & 39.47 & & & \\
\hline 86 & 17.00 & -20.61 & -11.07 & 44.05 & & & & & \\
\hline 87 & 16.00 & -19.30 & -10.91 & 43.28 & & & & & \\
\hline 88 & 17.30 & -17.93 & -11.15 & 43.38 & & & & & \\
\hline 89 & 15.50 & -21.26 & -11.56 & 43.22 & -25.52 & 38.40 & -10.84 & 43.94 & -0.28 \\
\hline 90 & 15.70 & -20.02 & -11.55 & 42.81 & & & & & \\
\hline 91 & 15.40 & -19.91 & -11.16 & 43.04 & -25.16 & 38.19 & -10.22 & 43.98 & -0.31 \\
\hline 92 & 15.50 & -19.48 & -11.14 & 42.93 & -25.15 & 38.06 & & & \\
\hline 93 & 15.94 & -20.80 & -10.82 & 43.95 & -25.51 & 38.41 & & & \\
\hline 94 & 15.00 & -20.16 & -11.36 & 42.79 & & & -10.83 & 43.31 & -0.54 \\
\hline 95 & 15.30 & -20.88 & -11.41 & 43.15 & -25.32 & 38.38 & & & \\
\hline 96 & 16.09 & -19.25 & -11.71 & 42.50 & & & & & \\
\hline 97 & 15.29 & -19.65 & -11.61 & 42.44 & -24.56 & 38.64 & -9.68 & 44.37 & -0.18 \\
\hline 98 & 13.80 & -20.62 & -11.43 & 42.41 & -24.91 & 38.08 & -10.17 & 43.68 & -0.19 \\
\hline 99 & 15.40 & -19.91 & -11.44 & 42.76 & & & & & \\
\hline 100 & 15.60 & -21.02 & -11.57 & 43.16 & -23.03 & 40.84 & & & \\
\hline 101 & 15.70 & -19.21 & -11.37 & 42.67 & & & & & \\
\hline 102 & 17.00 & -20.95 & -11.45 & 43.81 & -23.96 & 40.44 & & & \\
\hline 103 & 16.50 & -19.90 & -10.83 & 43.81 & -23.11 & 40.68 & & & \\
\hline 104 & 14.30 & -20.36 & -11.65 & 42.30 & -25.12 & 37.96 & & & \\
\hline 105 & 14.49 & -19.73 & -11.60 & 42.17 & -24.90 & 38.02 & & & \\
\hline 106 & 14.66 & -20.63 & -11.64 & 42.55 & -24.52 & 38.82 & -10.49 & 43.70 & -0.42 \\
\hline 107 & 13.30 & -21.00 & -11.45 & 42.70 & -24.86 & 38.09 & -10.37 & 43.43 & -0.41 \\
\hline 108 & 14.54 & -19.07 & -11.25 & 42.27 & -25.48 & 37.19 & -10.65 & 42.87 & -0.56 \\
\hline 109 & 17.00 & -21.02 & -11.06 & 44.23 & -23.05 & 41.38 & & & \\
\hline 110 & 14.90 & -21.80 & -11.29 & 43.47 & -24.47 & 39.43 & & & \\
\hline
\end{tabular}

$\mathrm{m}_{\mathrm{B}}, \mathrm{M}_{\mathrm{B}}$ in mag.

$\mathrm{f}_{\mathrm{X}}, \mathrm{f}_{\mathrm{FIR}}$ in erg s${ }^{-1} \mathrm{~cm}^{-2} ; \mathrm{f}_{R}$ in erg s $\mathrm{s}^{-1} \mathrm{~cm}^{-2} \mathrm{~Hz}^{-1}$

$\mathrm{L}_{\mathrm{X}}, v \mathrm{~L}_{\mathrm{R}}, \mathrm{L}_{\mathrm{FIR}}$ in erg $\mathrm{s}^{-1}$ 


\begin{tabular}{|c|c|c|c|c|c|c|c|c|c|}
\hline Nr. & $\mathrm{m}_{\mathrm{B}}$ & $\mathrm{M}_{\mathrm{B}}$ & $\log f_{X}$ & $\log L_{X}$ & $\log \mathrm{f}_{R}$ & $\log v L_{R}$ & $\log f_{\mathrm{FIR}}$ & $\log \mathrm{L}_{\mathrm{FIR}}$ & $\mathrm{FIR}_{\mathrm{col}}$ \\
\hline 111 & 14.20 & -21.13 & -11.63 & 42.93 & -25.03 & 38.33 & -10.31 & 43.90 & -0.46 \\
\hline 112 & 13.00 & -21.33 & -11.75 & 42.41 & & & & & \\
\hline 113 & 14.90 & -21.56 & -11.62 & 43.04 & -25.48 & 38.33 & & & \\
\hline 114 & 14.60 & -20.79 & -11.34 & 43.25 & -23.54 & 40.38 & & & \\
\hline 115 & 15.02 & -20.06 & -11.64 & 42.47 & & & & & \\
\hline 116 & 13.70 & -20.59 & -10.81 & 42.98 & -23.31 & 40.17 & & & \\
\hline 117 & 14.60 & -20.46 & -11.44 & 43.02 & & & & & \\
\hline 118 & 15.60 & -21.43 & -10.74 & 44.15 & -25.32 & 38.72 & & & \\
\hline 119 & 14.50 & -20.47 & -11.73 & 42.34 & -25.49 & 37.72 & & & \\
\hline 120 & 15.20 & -20.32 & -11.43 & 42.86 & & & & & \\
\hline 121 & 13.88 & -21.36 & -11.72 & 42.45 & -25.04 & 38.28 & & & \\
\hline 122 & 14.18 & -20.71 & -11.63 & 42.30 & -24.77 & 38.41 & -10.53 & 43.50 & -0.68 \\
\hline 123 & 14.42 & -20.36 & -11.69 & 42.40 & & & & & \\
\hline 124 & 13.82 & -21.92 & -11.57 & 42.80 & -25.31 & 38.21 & & & \\
\hline 125 & 13.90 & -20.68 & -11.55 & 42.36 & & & & & \\
\hline 126 & 14.60 & -20.74 & -11.74 & 42.83 & & & & & \\
\hline 127 & 14.20 & -23.20 & -11.27 & 43.77 & -24.50 & 39.68 & & & \\
\hline 128 & 17.60 & -19.49 & -12.01 & 42.90 & -24.72 & 39.34 & & & \\
\hline 129 & 14.60 & -21.00 & -11.37 & 43.30 & & & & & \\
\hline 130 & 14.20 & -22.78 & -11.13 & 44.10 & & & -10.32 & 44.55 & -0.28 \\
\hline 131 & 13.30 & -21.28 & -11.50 & 42.77 & -24.44 & 38.62 & -10.04 & 43.87 & -0.56 \\
\hline 132 & 14.15 & -19.68 & -11.42 & 42.19 & & & & & \\
\hline 133 & 15.50 & -18.38 & -11.74 & 41.89 & & & & & \\
\hline 134 & 15.40 & -20.99 & -11.54 & 43.10 & -24.79 & 38.99 & & & \\
\hline 135 & 15.70 & -21.42 & -11.20 & 43.73 & -23.97 & 40.10 & & & \\
\hline 136 & 15.30 & -20.60 & -11.57 & 43.23 & & & & & \\
\hline 137 & 14.60 & -20.99 & -11.37 & 43.30 & & & & & \\
\hline 138 & 15.99 & -20.88 & -10.98 & 43.85 & & & & & \\
\hline 139 & 15.64 & -20.04 & -11.51 & 42.84 & & & & & \\
\hline 140 & 14.23 & -22.31 & -11.07 & 43.62 & -22.86 & 40.97 & & & \\
\hline 141 & 15.90 & -22.04 & -11.32 & 43.94 & & & & & \\
\hline 142 & 12.33 & -22.09 & -11.12 & 42.73 & & & & & \\
\hline 143 & 15.00 & -21.75 & -11.39 & 43.39 & -23.23 & 41.24 & & & \\
\hline 144 & 16.20 & -18.98 & -11.49 & 42.66 & & & & & \\
\hline 145 & 15.30 & -21.51 & -11.35 & 43.45 & & & & & \\
\hline 146 & 15.60 & -20.78 & -11.56 & 43.06 & -25.03 & 38.74 & & & \\
\hline 147 & 14.40 & -22.16 & -11.54 & 43.16 & & & -10.61 & 44.09 & -0.37 \\
\hline 148 & 13.14 & -21.23 & -11.59 & 42.23 & & & & & \\
\hline 149 & 14.20 & -21.36 & -11.62 & 42.68 & & & & & \\
\hline 150 & 15.40 & -20.75 & -11.59 & 42.95 & & & & & \\
\hline 151 & 14.20 & -22.47 & -11.27 & 43.48 & & & & & \\
\hline 152 & 15.11 & -20.58 & -11.73 & 42.62 & & & -10.47 & 43.89 & -0.31 \\
\hline 153 & 15.60 & -21.30 & -11.75 & 43.45 & & & & & \\
\hline 154 & 18.10 & -19.13 & -12.01 & 42.96 & -24.92 & 39.20 & & & \\
\hline
\end{tabular}

$\mathrm{m}_{\mathrm{B}}, \mathrm{M}_{\mathrm{B}}$ in mag.

$\mathrm{f}_{\mathrm{X}}, \mathrm{f}_{\mathrm{FIR}}$ in erg s${ }^{-1} \mathrm{~cm}^{-2} ; \mathrm{f}_{R}$ in erg s $\mathrm{s}^{-1} \mathrm{~cm}^{-2} \mathrm{~Hz}^{-1}$

$\mathrm{L}_{\mathrm{X}}, v \mathrm{~L}_{\mathrm{R}}, \mathrm{L}_{\mathrm{FIR}}$ in erg $\mathrm{s}^{-1}$ 
Tabelle 8.2: Ergebnisse der optischen Spektroskopie des Samples gemäß der in Kapitel 3 beschrieben Messungen. Neben den Halbwertsbreiten FWHM und Flüssen $f_{\lambda}$ der breiten $\mathrm{H} \alpha$ - und H $\beta$-Komponenten sowie dem Mittelwert der Halbwertsbreiten (MW) sind das Balmerdekrement BD, die Rotverschiebung z und Distanz der Galaxie sowie der Galaxien-Typ aufgelistet. Anders als alle übrigen hier gelisteten Meßwerte können die $H \alpha$ - und $H \beta$-Flüsse Ungenauigkeiten durch nicht photometrische Beobachtungsbedingungen aufweisen. Die in dieser Arbeit verwendeten Flussverhältnisse sind unabhängig von solchen Ungenauigkeiten. Die Tabellennummerierung ist wie in den Tabellen 2.3 und 3.1 eingeführt.

\begin{tabular}{|c|c|c|c|c|c|c|c|c|c|}
\hline Nr. & $\begin{array}{c}\text { FMHM } \\
\text { H } \alpha\end{array}$ & $\begin{array}{c}\text { FWHM } \\
\text { H } \beta\end{array}$ & $\begin{array}{c}\text { FWHM } \\
\text { MW }\end{array}$ & $\begin{array}{l}\mathrm{f}_{\lambda} \\
\mathrm{H} \alpha\end{array}$ & $\begin{array}{l}\mathrm{f}_{\lambda} \\
\mathrm{H} \beta\end{array}$ & $\begin{array}{l}\text { BD } \\
\text { kor. }\end{array}$ & z & Distanz & Тyр \\
\hline 1 & 4898 & 5740 & 5319 & 1294.9 & 222.36 & 5.82 & 0.0370 & 144 & VBLG \\
\hline 2 & 13539 & 17468 & 15504 & 35.27 & 12.33 & 2.86 & 0.0590 & 226 & VBLG \\
\hline 3 & 4964 & 8392 & 6678 & 2697.6 & 586.09 & 4.60 & 0.2970 & 975 & VBLG \\
\hline 4 & 8088 & 11010 & 9549 & 2837.1 & 641.83 & 4.42 & 0.0680 & 259 & VBLG \\
\hline 5 & 6499 & 5998 & 6249 & 282.86 & 38.37 & 7.37 & 0.3060 & 999 & VBLG \\
\hline 6 & 10384 & & 10384 & 310.47 & & & 0.0860 & 323 & VBLG \\
\hline 7 & 5854 & 6820 & 6337 & 282.75 & 84.92 & 3.33 & 0.2640 & 884 & VBLG \\
\hline 8 & 11889 & 14305 & 13097 & 1445.7 & 308.00 & 4.69 & 0.0590 & 226 & VBLG \\
\hline 9 & 11221 & 12862 & 12042 & 1216.6 & 265.01 & 4.59 & 0.0570 & 219 & VBLG \\
\hline 10 & 7760 & & 7760 & 961.70 & 150.00 & 6.41 & 0.0570 & 219 & VBLG \\
\hline 11 & 10843 & 10174 & 10509 & 594.40 & 172.50 & 3.45 & 0.3230 & 1044 & VBLG \\
\hline 12 & 9830 & 15332 & 12581 & 283.82 & 96.48 & 2.94 & 0.1090 & 403 & VBLG \\
\hline 13 & & 12884 & 12884 & & 186.15 & & 0.4620 & 1383 & VBLG \\
\hline 14 & 9436 & 3791 & 6614 & 842.40 & 210.47 & 4.00 & 0.2980 & 977 & VBLG \\
\hline 15 & 12202 & 14830 & 13516 & 7993.8 & 3037.3 & 2.63 & 0.0440 & 170 & VBLG \\
\hline 16 & 16369 & 13423 & 14896 & 669.27 & 140.72 & 4.76 & 0.0250 & 98 & VBLG \\
\hline 17 & 6745 & 4777 & 5761 & 1771.4 & 716.10 & 2.80 & 0.0368 & 143 & VBLG \\
\hline 18 & 13350 & 12547 & 12949 & 567.00 & 190.00 & 2.98 & 0.0520 & 200 & VBLG \\
\hline 19 & 9358 & 13129 & 11244 & 1559.0 & 584.00 & 2.67 & 0.0670 & 255 & VBLG \\
\hline 20 & 7953 & 6128 & 7041 & 197.86 & 45.23 & 4.37 & 0.4750 & 1412 & VBLG \\
\hline 21 & 7840 & 8960 & 8400 & 1033.0 & 284.00 & 3.64 & 0.1290 & 471 & VBLG \\
\hline 22 & 9462 & 12248 & 10855 & 1589.6 & 356.71 & 4.46 & 0.0480 & 185 & VBLG \\
\hline 23 & 6255 & 3572 & 4914 & 758.00 & 244.86 & 3.10 & 0.0490 & 189 & VBLG \\
\hline 24 & 6369 & 11376 & 8873 & 3040.9 & 915.20 & 3.32 & 0.0090 & 36 & VBLG \\
\hline 25 & 5775 & 6161 & 5968 & 627.60 & 222.44 & 2.82 & 0.0890 & 334 & VBLG \\
\hline 26 & 4436 & 5529 & 4983 & 375.54 & 143.94 & 2.61 & 0.1420 & 514 & VBLG \\
\hline 27 & 4206 & & 4206 & 97.57 & & & 0.1180 & 434 & VBLG \\
\hline 28 & 4738 & 7496 & 6117 & 570.69 & 188.99 & 3.02 & 0.0890 & 334 & VBLG \\
\hline 29 & 8737 & 10667 & 9702 & 109.38 & 51.73 & 2.80 & 0.2900 & 956 & VBLG \\
\hline 30 & & 12235 & 12235 & & 91.71 & & 0.4570 & 1371 & VBLG \\
\hline 31 & & 4620 & 4620 & & 11.27 & & 0.4250 & 1297 & VBLG \\
\hline 32 & & 7205 & 7205 & & 48.40 & & 0.6690 & 1806 & VBLG \\
\hline 33 & & 3051 & 3051 & & 34.52 & & 0.4080 & 1257 & VBLG \\
\hline
\end{tabular}

FWHM in $\mathrm{km} \mathrm{s}^{-1}$

$\mathrm{f}_{\lambda}$ in $10^{-15} \mathrm{erg} \mathrm{s}^{-1} \mathrm{~cm}^{-2} \AA^{-1}$

Distanz in Mpc 


\begin{tabular}{|c|c|c|c|c|c|c|c|c|c|}
\hline Nr. & $\begin{array}{c}\text { FMHM } \\
\mathrm{H} \alpha\end{array}$ & $\begin{array}{c}\text { FWHM } \\
\mathrm{H} \beta\end{array}$ & $\begin{array}{c}\text { FWHM } \\
\text { MW }\end{array}$ & $\begin{array}{l}\mathrm{f}_{\lambda} \\
\mathrm{H} \alpha\end{array}$ & $\begin{array}{l}\mathrm{f}_{\lambda} \\
\mathrm{H} \beta\end{array}$ & $\begin{array}{l}\text { BD } \\
\text { kor. }\end{array}$ & $\mathrm{z}$ & Distanz & Тур \\
\hline 34 & 11712 & 15886 & 13799 & 73.11 & 26.22 & 2.79 & 0.3220 & 1041 & VBLG \\
\hline 35 & & 4399 & 4399 & & 55.66 & & 0.5740 & 1622 & VBLG \\
\hline 36 & 12899 & 6491 & 9695 & 1297.9 & 106.76 & 12.16 & 0.5010 & 1469 & VBLG \\
\hline 37 & 7930 & 9196 & 8563 & 674.62 & 154.05 & 4.38 & 0.2000 & 697 & VBLG \\
\hline 38 & 9148 & 5354 & 7251 & 314.00 & 80.42 & 3.90 & 0.2130 & 736 & VBLG \\
\hline 39 & 14274 & 15012 & 14643 & 345.00 & 104.78 & 3.29 & 0.1790 & 632 & VBLG \\
\hline 40 & 4637 & 8062 & 6350 & 34.51 & 5.98 & 5.77 & 0.0720 & 273 & VBLG \\
\hline 41 & 14490 & 10782 & 12636 & 201.31 & 39.13 & 5.14 & 0.3660 & 1154 & VBLG \\
\hline 42 & 5937 & 2825 & 4381 & 502.60 & 81.11 & 6.20 & 0.1310 & 477 & VBLG \\
\hline 43 & 2209 & & 2209 & 1031.7 & & & 0.0250 & 98 & VBLG \\
\hline 44 & 12440 & 6199 & 9320 & 125.10 & 45.39 & 2.76 & 0.1750 & 619 & VBLG \\
\hline 45 & & & & & & & & & BL Lac \\
\hline 46 & & & & & & & & & BL Lac \\
\hline 47 & & & & & & & & & BL Lac \\
\hline 48 & & & & & & & & & BL Lac \\
\hline 49 & & & 2791 & & & & 0.6785 & 1824 & QSO \\
\hline 50 & 1974 & 2617 & 2296 & 182.44 & 86.25 & 2.80 & 0.0305 & 119 & Sy1 \\
\hline 51 & 5617 & 7185 & 6401 & 24.03 & 13.94 & 2.80 & 0.0473 & 183 & Sy1 \\
\hline 52 & 2194 & 2028 & 2111 & 18.49 & 3.98 & 4.65 & 0.0388 & 151 & Sy1 \\
\hline 53 & 8250 & & 8250 & & & & 0.0451 & 174 & Sy1 \\
\hline 54 & 3450 & & 3450 & & & & 0.0269 & 106 & Sy1 \\
\hline 55 & 6983 & 6771 & 6877 & 423.54 & 148.04 & 2.86 & 0.0848 & 319 & Sy1 \\
\hline 56 & 2000 & & 2000 & & & & 0.0257 & 101 & Sy1 \\
\hline 57 & 2750 & & 2750 & & & & 0.0609 & 233 & Sy1 \\
\hline 58 & 3400 & & 3400 & & & & 0.0405 & 157 & Sy1 \\
\hline 59 & 3000 & & 3000 & & & & 0.0235 & 92 & Sy1 \\
\hline 60 & 3747 & 6029 & 4888 & 152.87 & 53.47 & 2.86 & 0.0241 & 94 & Sy1 \\
\hline 61 & 16000 & & 16000 & & & & 0.0590 & 226 & Sy1 \\
\hline 62 & 4100 & & 4100 & & & & 0.0248 & 97 & Sy1 \\
\hline 63 & 3620 & 4443 & 4032 & 439.94 & 142.05 & 3.10 & 0.0225 & 88 & Sy1 \\
\hline 64 & 4450 & & 4450 & & & & 0.0137 & 54 & Sy1 \\
\hline 65 & 4000 & & 4000 & & & & 0.0396 & 154 & Sy1 \\
\hline 66 & 5900 & & 5900 & & & & 0.0330 & 129 & Sy1 \\
\hline 67 & 3660 & 3662 & 3661 & 11.53 & 6.87 & 2.80 & 0.0739 & 280 & Sy1 \\
\hline 68 & 2100 & & 2100 & & & & 0.0263 & 103 & Sy1 \\
\hline 69 & 4200 & & 4200 & & & & 0.0325 & 127 & Sy1 \\
\hline 70 & 3317 & 3497 & 3407 & 112.07 & 38.37 & 2.92 & 0.0192 & 76 & Sy1 \\
\hline 71 & 2707 & 3558 & 3133 & 31.28 & 6.30 & 4.96 & 0.0703 & 267 & Sy1 \\
\hline 72 & 2151 & 2281 & 2216 & 91.54 & 32.94 & 2.78 & 0.0379 & 147 & Sy1 \\
\hline 73 & 4500 & & 4500 & & & & 0.0159 & 63 & Sy1 \\
\hline 74 & 5400 & & 5400 & & & & 0.0358 & 139 & Sy1 \\
\hline 75 & 2850 & & 2850 & & & & 0.0284 & 111 & Sy1 \\
\hline 76 & 3700 & & 3700 & & & & 0.0760 & 287 & Sy1 \\
\hline
\end{tabular}

FWHM in $\mathrm{km} \mathrm{s}^{-1}$

$\mathrm{f}_{\lambda}$ in $10^{-15} \mathrm{erg} \mathrm{s}^{-1} \mathrm{~cm}^{-2} \AA^{-1}$

Distanz in Mpc 


\begin{tabular}{|c|c|c|c|c|c|c|c|c|c|}
\hline Nr. & $\begin{array}{l}\text { FMHM } \\
\text { Ho }\end{array}$ & $\begin{array}{c}\text { FWHM } \\
\mathrm{H} \beta\end{array}$ & $\begin{array}{c}\text { FWHM } \\
\text { MW }\end{array}$ & $\begin{array}{l}\mathrm{f}_{\lambda} \\
\mathrm{H} \alpha\end{array}$ & $\mathrm{f}_{\lambda}$ & $\begin{array}{l}\text { BD } \\
\text { kor. }\end{array}$ & $\mathrm{z}$ & Distanz & Typ \\
\hline 77 & & 6000 & 6000 & & 8.67 & & 0.1362 & 495 & Sy 1 \\
\hline 78 & 3928 & 4766 & 4347 & 117.80 & 81.08 & 2.80 & 0.0406 & 158 & Sy1 \\
\hline 79 & 2950 & & 2950 & & & & 0.0286 & 112 & Sy 1 \\
\hline 80 & 5500 & & 5500 & & & & 0.0334 & 130 & Sy 1 \\
\hline 81 & 2500 & & 2500 & & & & 0.1288 & 470 & Sy 1 \\
\hline 82 & 1950 & & 1950 & & & & 0.0413 & 160 & Sy1 \\
\hline 83 & 2048 & 1427 & 1738 & 61.56 & 11.45 & 5.37 & 0.0134 & 53 & NLS1 \\
\hline 84 & 1411 & & 1411 & 106.30 & 37.44 & 2.84 & 0.0435 & 168 & NLS1 \\
\hline 85 & 1480 & 1240 & 1360 & 20.74 & 4.67 & 4.44 & 0.0913 & 342 & NLS1 \\
\hline 86 & 1176 & 1330 & 1253 & 38.64 & 14.52 & 2.66 & 0.0815 & 307 & NLS1 \\
\hline 87 & 2159 & 1884 & 2022 & 61.38 & 16.34 & 3.76 & 0.0285 & 112 & NLS1 \\
\hline 88 & 2100 & & 2100 & & & & 0.0276 & 108 & NLS1 \\
\hline 89 & 3597 & 5676 & 4637 & 44.42 & 18.75 & 2.80 & 0.0554 & 213 & Sy1.5 \\
\hline 90 & 1652 & 1803 & 1728 & 11.09 & 2.73 & 4.06 & 0.0345 & 134 & Sy 1.5 \\
\hline 91 & 6462 & 8702 & 7582 & 105.93 & 44.73 & 2.80 & 0.0287 & 112 & Sy1.5 \\
\hline 92 & 5216 & & 5216 & 663.74 & & & 0.0246 & 97 & Sy1.5 \\
\hline 93 & 4712 & 5703 & 5208 & 168.95 & 48.30 & 3.50 & 0.0549 & 211 & Sy1.5 \\
\hline 94 & 7454 & 9289 & 8372 & 151.51 & 50.94 & 2.97 & 0.0268 & 105 & Sy1.5 \\
\hline 95 & 2756 & 2774 & 2765 & 47.23 & 19.45 & 2.80 & 0.0426 & 165 & Sy1.5 \\
\hline 96 & 2404 & 5113 & 3759 & 28.70 & 4.66 & 6.16 & 0.0291 & 114 & Sy1.5 \\
\hline 97 & 4309 & & 4309 & 69.67 & & & 0.0242 & 95 & Sy1.8 \\
\hline 98 & 5789 & 9916 & 7853 & 55.90 & 21.04 & 2.66 & 0.0190 & 75 & Sy 1.8 \\
\hline 99 & 4087 & & 4087 & 19.39 & & & 0.0287 & 112 & Sy1.9 \\
\hline 100 & 3642 & & 3642 & 13.28 & & & 0.0522 & 201 & Sy 1.9 \\
\hline 101 & 6787 & & 6787 & 12.57 & & & 0.0239 & 94 & Sy 1.9 \\
\hline 102 & 8162 & & 8162 & 5.22 & & & 0.0953 & 356 & Sy 1.9 \\
\hline 103 & 3700 & & 3700 & 14.42 & & & 0.0471 & 182 & Sy 1.9 \\
\hline 104 & 4022 & & 4022 & 89.56 & & & 0.0213 & 84 & Sy 1.9 \\
\hline 105 & 5655 & & 5655 & 65.11 & & & 0.0174 & 69 & Sy 1.9 \\
\hline 106 & & & & & & & 0.0284 & 111 & Sy2 \\
\hline 107 & & & & & & & 0.0181 & 71 & Sy2 \\
\hline 108 & & & & & & & 0.0132 & 52 & Sy2 \\
\hline 109 & & & & & & & 0.0982 & 366 & Sy2 \\
\hline 110 & & & & & & & 0.0539 & 207 & Sy2 \\
\hline 111 & & & & & & & 0.0289 & 113 & Sy2 \\
\hline 112 & & & & & & & 0.0183 & 72 & LLAGN \\
\hline 113 & & & & & & & 0.0484 & 187 & LLAGN \\
\hline 114 & & & & & & & 0.0298 & 116 & LLAGN \\
\hline 115 & & & & & & & 0.0258 & 101 & LLAGN \\
\hline 116 & & & & & & & 0.0179 & 71 & LLAGN \\
\hline 117 & & & & & & & 0.0256 & 100 & LLAGN \\
\hline 118 & & & & & & & 0.0628 & 240 & LLAGN \\
\hline 119 & & & & & & & 0.0245 & 96 & LLAGN \\
\hline
\end{tabular}

FWHM in $\mathrm{km} \mathrm{s}^{-1}$

$\mathrm{f}_{\lambda}$ in $10^{-15} \mathrm{erg} \mathrm{s}^{-1} \mathrm{~cm}^{-2} \AA^{-1}$

Distanz in Mpc 


\begin{tabular}{|c|c|c|c|c|c|c|c|c|c|}
\hline Nr. & $\begin{array}{c}\text { FMHM } \\
\mathrm{H} \alpha\end{array}$ & $\begin{array}{c}\text { FWHM } \\
\mathrm{H} \beta\end{array}$ & $\begin{array}{c}\text { FWHM } \\
\text { MW }\end{array}$ & $\begin{array}{l}\mathrm{f}_{\lambda} \\
\mathrm{H} \alpha\end{array}$ & $\begin{array}{l}\mathrm{f}_{\lambda} \\
\mathrm{H} \beta\end{array}$ & $\begin{array}{l}\text { BD } \\
\text { kor. }\end{array}$ & $\mathrm{z}$ & Distanz & Typ \\
\hline 120 & & & & & & & 0.0316 & 123 & LLAGN \\
\hline 121 & & & & & & & 0.0277 & 108 & LLAGN \\
\hline 122 & & & & & & & 0.0236 & 93 & LLAGN \\
\hline 123 & & & & & & & 0.0225 & 88 & LLAGN \\
\hline 124 & & & & & & & 0.0348 & 136 & LLAGN \\
\hline 125 & & & & & & & 0.0205 & 81 & LLAGN \\
\hline 126 & & & & & & & 0.0290 & 114 & LLAGN \\
\hline 127 & & & & & & & 0.0742 & 281 & LLAGN \\
\hline 128 & & & & & & & 0.0646 & 246 & LLAGN \\
\hline 129 & & & & & & & 0.0328 & 128 & LINER \\
\hline 130 & & & & & & & 0.0614 & 235 & LINER \\
\hline 131 & & & & & & & 0.0205 & 81 & LINER \\
\hline 132 & & & & & & & 0.0145 & 57 & HII \\
\hline 133 & & & & & & & 0.0149 & 59 & HII \\
\hline 134 & & & & & & & 0.0469 & 181 & HII \\
\hline 135 & & & & & & & 0.0655 & 250 & inaktiv \\
\hline 136 & & & & & & & 0.0376 & 146 & inaktiv \\
\hline 137 & & & & & & & 0.0326 & 127 & inaktiv \\
\hline 138 & & & & & & & 0.0583 & 223 & inaktiv \\
\hline 139 & & & & & & & 0.0339 & 132 & inaktiv \\
\hline 140 & & & & & & & 0.0502 & 193 & inaktiv \\
\hline 141 & & & & & & & 0.0949 & 354 & inaktiv \\
\hline 142 & & & & & & & 0.0191 & 75 & inaktiv \\
\hline 143 & & & & & & & 0.0553 & 212 & inaktiv \\
\hline 144 & & & & & & & 0.0270 & 106 & inaktiv \\
\hline 145 & & & & & & & 0.0567 & 217 & inaktiv \\
\hline 146 & & & & & & & 0.0466 & 180 & inaktiv \\
\hline 147 & & & & & & & 0.0506 & 195 & inaktiv \\
\hline 148 & & & & & & & 0.0186 & 73 & inaktiv \\
\hline 149 & & & & & & & 0.0321 & 125 & inaktiv \\
\hline 150 & & & & & & & 0.0420 & 163 & inaktiv \\
\hline 151 & & & & & & & 0.0534 & 205 & inaktiv \\
\hline 152 & & & & & & & 0.0341 & 133 & inaktiv \\
\hline 153 & & & & & & & 0.0590 & 226 & inaktiv \\
\hline 154 & & & & & & & 0.0688 & 262 & inaktiv \\
\hline
\end{tabular}

FWHM in $\mathrm{km} \mathrm{s}^{-1}$

$\mathrm{f}_{\lambda}$ in $10^{-15} \mathrm{erg} \mathrm{s}^{-1} \mathrm{~cm}^{-2} \AA^{-1}$

Distanz in Mpc 
Tabelle 8.3: Liste der Galaxien, bei denen breite Balmerlinien durch zwei Komponenten angepasst wurden. Es ist für $\mathrm{H} \alpha$ und $\mathrm{H} \beta$ jeweils die Halbwertsbreite FWHM der breiten (b) und der sehr breiten (v) Komponenten sowie der relative Flussanteil der sehr breiten Komponente $\mathrm{f}_{v} / \mathrm{f}_{\text {tot }}$ aufgelistet. Ferner ist die flussgewichtet gemittelte Halbwertsbreite $\left(\mathrm{FWHM}_{m} \mathrm{MW}\right.$ nach Gleichung 6.2 und der Mittelwert der relativen Flussanteile der sehr breiten H $\alpha$ - und H $\beta$ Komponenten aufgeführt (s. Abschnitt 6.1).

\begin{tabular}{|c|c|c|c|c|c|c|c|c|}
\hline Nr. & $\begin{array}{c}\text { FMHM } \\
\text { H } \alpha \text { b }\end{array}$ & $\begin{array}{c}\text { FWHM } \\
\text { H } \alpha \text { v }\end{array}$ & $\begin{array}{c}\mathrm{f}_{v} / \mathrm{f}_{t o t} \\
\mathrm{H} \alpha\end{array}$ & $\begin{array}{c}\text { FMHM } \\
\text { H } \beta \text { b }\end{array}$ & $\begin{array}{c}\text { FWHM } \\
\text { H } \beta \text { v }\end{array}$ & $\begin{array}{c}\mathrm{f}_{v} / \mathrm{f}_{t o t} \\
\mathrm{H} \beta\end{array}$ & $\begin{array}{c}\mathrm{FWHM}_{m} \\
\mathrm{MW}\end{array}$ & $\begin{array}{l}\mathrm{f}_{v} / \mathrm{f}_{t o t} \\
\mathrm{MW}\end{array}$ \\
\hline 3 & & & & 2686 & 8392 & 0.75 & 5957 & 0.75 \\
\hline 6 & 2285 & 10384 & 0.84 & & & & 9062 & 0.84 \\
\hline 7 & 5854 & 13407 & 0.26 & & & & 7308 & 0.26 \\
\hline 10 & 3043 & 7760 & 0.63 & & & & 5995 & 0.63 \\
\hline 11 & 3054 & 10843 & 0.65 & 2932 & 10174 & 0.73 & 8140 & 0.69 \\
\hline 14 & 2971 & 9436 & 0.35 & & & & 4519 & 0.35 \\
\hline 17 & 2464 & 6744 & 0.69 & 4777 & 10345 & 0.41 & 6249 & 0.55 \\
\hline 18 & 4326 & 13341 & 0.38 & 4723 & 12538 & 0.57 & 8457 & 0.47 \\
\hline 19 & 3595 & 9358 & 0.48 & 4465 & 13129 & 0.54 & 7771 & 0.51 \\
\hline 20 & 1498 & 7953 & 0.75 & 1448 & 6128 & 0.74 & 5610 & 0.74 \\
\hline 22 & 2611 & 9462 & 0.80 & 3582 & 12248 & 0.80 & 9293 & 0.80 \\
\hline 23 & 2183 & 6255 & 0.55 & & & & 3991 & 0.55 \\
\hline 24 & 2410 & 6369 & 0.71 & 4164 & 11376 & 0.66 & 7079 & 0.69 \\
\hline 25 & 2174 & 5775 & 0.76 & & & & 5535 & 0.76 \\
\hline 28 & 1642 & 4738 & 0.58 & 2239 & 7496 & 0.52 & 4196 & 0.55 \\
\hline 29 & 1940 & 8737 & 0.64 & 1665 & 10667 & 0.78 & 7481 & 0.71 \\
\hline 30 & & & & 3433 & 12235 & 0.73 & 9826 & 0.73 \\
\hline 33 & & & & 3051 & 13095 & 0.75 & 10617 & 0.75 \\
\hline 35 & & & & 4399 & 12424 & 0.68 & 9845 & 0.68 \\
\hline 38 & 1371 & 9148 & 0.80 & & & & 6488 & 0.80 \\
\hline 39 & 4223 & 14274 & 0.49 & 4425 & 15012 & 0.78 & 10932 & 0.64 \\
\hline 41 & 6898 & 14490 & 0.72 & & & & 11568 & 0.72 \\
\hline 42 & 2090 & 5934 & 0.40 & & & & 3231 & 0.40 \\
\hline 55 & 2738 & 6983 & 0.80 & & & & 6443 & 0.80 \\
\hline 67 & 1467 & 3660 & 0.53 & & & & 3147 & 0.53 \\
\hline 71 & 2707 & 7183 & 0.48 & & & & 4203 & 0.48 \\
\hline 94 & 2280 & 7454 & 0.62 & 2214 & 9289 & 0.80 & 6707 & 0.71 \\
\hline 101 & 1685 & 6788 & 0.70 & & & & 5242 & 0.70 \\
\hline
\end{tabular}

FWHM in $\mathrm{km} \mathrm{s}^{-1}$ 
Tabelle 8.4: Säulendichte neutralen Wasserstoffs $\mathrm{N}_{H}$ nach Dickey \& Lockman (1990) und Röntgenzählrate CR für die Galaxien des Samples. Für einige VBLG liegen keine Röntgendaten vor (s. Abschnitt 4.4.3).

\begin{tabular}{rlcc}
\hline \hline Nr. & Name & $\mathrm{N}_{H}$ & $\mathrm{CR}$ \\
\hline 1 & 1E04124-080 & 2.20 & 0.051 \\
2 & 1E0450.3 & 1.19 & \\
3 & 1E1821+643 & 0.17 & 1.071 \\
4 & 1E2251-178 & 0.71 & 1.037 \\
5 & 3C109.0 & 1.40 & 0.112 \\
6 & 3C227 & 4.02 & 0.017 \\
7 & 3C323.1 & 0.42 & 0.311 \\
8 & 3C382 & 0.50 & 2.000 \\
9 & 3C390.3 & 0.21 & 0.472 \\
10 & 3C445 & 8.86 & 0.017 \\
11 & 4 C11.72 & 1.57 & \\
12 & 4C29.06 & 0.55 & 0.202 \\
13 & 4 C31.30 & 0.51 & 0.114 \\
14 & 4 C31.63 & 0.61 & 0.242 \\
15 & $4 \mathrm{U} 0241+61$ & 0.28 & 0.411 \\
16 & Arp102B & 0.44 & 0.056 \\
17 & ESO 141-55 & & \\
18 & Mkn1152 & 0.98 & 0.945 \\
19 & Mkn304 & 1.13 & 0.014 \\
20 & Mkn380 & 0.13 & \\
21 & Mkn876 & 0.24 & 0.864 \\
22 & Mkn926 & 1.75 & 1.720 \\
23 & Mkn975 & 1.95 & \\
24 & NGC3516 & 0.20 & 0.140 \\
25 & PG0007+106 & 1.61 & 0.479 \\
26 & PG0026+12 & 1.85 & 0.469 \\
27 & PG0906+48 & 0.13 & 0.355 \\
28 & PG1411+442 & 0.33 & 0.033 \\
29 & PG1700+518 & 0.38 & \\
30 & PKS0130+242 & 0.64 & \\
31 & PKS0133+207 & 0.75 & 0.169 \\
32 & PKS0159-117 & 1.68 & 0.176 \\
33 & PKS0214+108 & 1.18 & 0.251 \\
34 & PKS0231+022 & 10.9 & 0.161 \\
35 & PKS0405-12 & 0.98 & 0.418 \\
36 & PKS2128-123 & 1.07 & 0.219 \\
37 & PKS2135-147 & 0.86 & 0.463 \\
38 & PKS2141+175 & 0.65 & 0.065 \\
39 & PKS2349-01 & 10.5 & 0.531 \\
40 & S10721 & 0.62 & 0.069 \\
\hline \hline
\end{tabular}

$\mathrm{N}_{H}$ in $10^{20} \mathrm{~cm}^{-2}$

$\mathrm{CR}$ in cts./s 


\begin{tabular}{llcc}
\hline \hline Nr. & Name & $\mathrm{N}_{H}$ & $\mathrm{CR}$ \\
\hline 41 & TON202 & 0.39 & 0.033 \\
42 & TON256 & 0.46 & 0.427 \\
43 & UGC12163 & 0.93 & 3.836 \\
44 & V396Her & 0.49 & 0.133 \\
45 & 1RXSJ201731.2-411452 & & \\
46 & PHL 1389 & 0.17 & 0.043 \\
47 & PMN J0630-2406 & 0.97 & 0.084 \\
48 & RX J011232.8-320140 & 0.21 & 0.033 \\
49 & RX J023454.8-293425 & 0.17 & 0.006 \\
50 & AM 2354-304 E & 0.14 & 0.057 \\
51 & CGCG 015-026 & 0.18 & 0.068 \\
52 & CGCG 049-106 & 0.34 & 0.107 \\
53 & CGCG 433-025 & 0.44 & 0.179 \\
54 & ESO 080- G 005 & 0.25 & 0.091 \\
55 & ESO 104-IG041A & 0.53 & 0.241 \\
56 & ESO 113- G 010 & 0.30 & 0.182 \\
57 & ESO 15- IG 011 & 0.70 & 0.274 \\
58 & ESO 209- G 012 & 2.68 & 0.163 \\
59 & ESO 244- G 017 & 0.24 & 0.305 \\
60 & ESO 328-36 S & 0.62 & 0.162 \\
61 & ESO 416- G 002 & 0.17 & 0.356 \\
62 & ESO 490- IG 026 & 1.09 & 0.273 \\
63 & ESO 511-30 & 0.49 & 1.221 \\
64 & ESO 548- G 081 & 0.28 & 0.258 \\
65 & ESO 552- G 039 & 0.21 & 0.106 \\
66 & ESO 602- G 031 & 0.22 & 0.422 \\
67 & Fairall 969 & 0.16 & 0.145 \\
68 & HCG 4a & 0.16 & 0.330 \\
69 & IC 5287 & 0.42 & 0.091 \\
70 & IRAS F20315-3047 & 0.52 & 0.167 \\
71 & KUG 0128+328 & 0.48 & 0.104 \\
72 & KUG 1618+410 & 0.10 & 0.510 \\
73 & MCG -01-13-025 & 0.41 & 0.281 \\
74 & MCG -02-12-050 & 0.60 & 0.066 \\
75 & MCG -02-14-009 & 0.91 & 0.307 \\
76 & MCG -02-59-006 & 0.27 & 0.177 \\
77 & MCG 1-59-85 X884 & 0.53 & 0.092 \\
78 & MCG 3-47-2 & 1.52 & 0.129 \\
79 & NGC 1218 & 0.73 & 0.175 \\
80 & NGC 427 & 0.21 & 0.153 \\
81 & PMN J0623-6436 & 0.56 & 0.417 \\
82 & VIII Zw 36 & 0.26 & 0.244 \\
83 & CGCG 374-029 & 0.81 & 0.055 \\
84 & CGCG 493-004 & 1.43 & 0.074 \\
85 & ESO 407-17 & & \\
\hline & & & \\
\hline & in & & \\
\hline
\end{tabular}

$\mathrm{N}_{H}$ in $10^{20} \mathrm{~cm}^{-2}$

$\mathrm{CR}$ in cts./s 


\begin{tabular}{rlcc}
\hline \hline Nr. & Name & $\mathrm{N}_{H}$ & CR \\
\hline 86 & IIZW 177 & 0.54 & 0.247 \\
87 & KUG 1618+402 & 0.08 & 0.356 \\
88 & NGC 7158 & 0.39 & 0.285 \\
89 & CGCG 163-074 & 0.11 & 0.079 \\
90 & CGCG 196-064 & 0.13 & 0.081 \\
91 & CGCG 425-034 & 0.69 & 0.203 \\
92 & CGCG 493-002 & 1.31 & 0.185 \\
93 & ESO 381-7 & 0.55 & 0.446 \\
94 & MCG 6-38-5 & 0.27 & 0.125 \\
95 & MRK 863 & 0.36 & 0.115 \\
96 & NPM1G-16.0443 & 0.76 & 0.057 \\
97 & CGCG 022-021 & 0.93 & 0.070 \\
98 & MCG -01-30-041 & 0.23 & 0.108 \\
99 & CGCG 023-021 & 0.62 & 0.105 \\
100 & CGCG 21-63 & 0.46 & 0.078 \\
101 & CGCG 399-005 & 1.45 & 0.123 \\
102 & PKS 0018-19 & 0.20 & 0.104 \\
103 & PKS 2331-24 & 0.17 & 0.437 \\
104 & UGC 12040 & 1.23 & 0.058 \\
105 & UGC 12282 & 1.22 & 0.065 \\
106 & ESO 409- 3 & 0.15 & 0.067 \\
107 & MCG -01-05-031 & 0.28 & 0.175 \\
108 & MCG -01-33-054 & 0.26 & 0.164 \\
109 & PKS 1928-34 & 0.76 & 0.254 \\
110 & PMN J0100-1517 & 0.17 & 0.151 \\
111 & UGC 3134 & 0.47 & 0.083 \\
112 & AM 0426-625 & 0.24 & 0.097 \\
113 & CGCG 375-033 & 0.50 & 0.071 \\
114 & ESO 254- G 017 & 0.51 & 0.213 \\
115 & ESO 399- 15 & 0.72 & 0.066 \\
116 & ESO 45- 11 & 0.81 & 0.449 \\
117 & IC 1867 & 1.18 & 0.083 \\
118 & MCG 6-37-23 & 0.21 & 0.520 \\
119 & NGC 5959 & 0.75 & 0.054 \\
120 & NGC 6159 & 0.10 & 0.108 \\
121 & NGC 6370 & 0.32 & 0.054 \\
122 & NGC 70 & 0.55 & 0.006 \\
123 & NGC 71 & 0.55 & 0.006 \\
124 & NGC 7749 & 0.14 & 0.079 \\
125 & UGC 11950 & 1.29 & 0.072 \\
126 & UGC 12492 & 0.42 & 0.069 \\
127 & UGC 32 & 0.53 & 0.148 \\
128 & VIIZW 608 & 0.18 & 0.028 \\
129 & ESO 122- IG 016 & 0.97 & 0.106 \\
130 & ESO 15- IG 011 b & 0.70 & 0.274 \\
\hline \hline & & & \\
\hline & I0 c-2 & & \\
\hline
\end{tabular}

$\mathrm{N}_{H}$ in $10^{20} \mathrm{~cm}^{-2}$

$\mathrm{CR}$ in cts./s 


\begin{tabular}{clcc}
\hline \hline Nr. & Name & $\mathrm{N}_{H}$ & CR \\
\hline 131 & NGC 1217 & 0.21 & 0.193 \\
132 & ESO 267-13 & 0.91 & 0.110 \\
133 & MCG 5-34-53 & 0.14 & 0.052 \\
134 & MCG 7-37-18 comp. & & \\
135 & CGCG 043-056 & 0.19 & 0.186 \\
136 & ESO 120- G 023 & 0.49 & 0.093 \\
137 & ESO 122- IG 016 b & 0.97 & 0.106 \\
138 & ESO 184- 68 & 0.39 & 0.309 \\
139 & ESO 290- 3 NE & 0.17 & 0.090 \\
140 & ESO 350- 15 & 0.17 & 0.249 \\
141 & ESO 600- 14 & 0.31 & 0.141 \\
142 & IC 4991 & & \\
143 & MCG -07-47-032 & 0.20 & 0.120 \\
144 & MCG 10-26-15 & 0.35 & 0.091 \\
145 & MCG 13-12-22 & 0.40 & 0.126 \\
146 & MCG 5-41-10 & 0.33 & 0.077 \\
147 & MCG 7-37-18 & 0.33 & 0.082 \\
148 & NGC 57 & 0.40 & 0.072 \\
149 & NGC 6160 & 0.10 & 0.069 \\
150 & NGC 6414 & 0.38 & 0.072 \\
151 & SGC 2005.3-5431 & 0.54 & 0.157 \\
152 & UGC 12804 & 0.44 & 0.051 \\
153 & UGC 716 & 0.41 & 0.069 \\
154 & VIIZW 608 comp. & 0.18 & 0.028 \\
\hline \hline
\end{tabular}

$\mathrm{N}_{H}$ in $10^{20} \mathrm{~cm}^{-2}$

$\mathrm{CR}$ in cts./s 

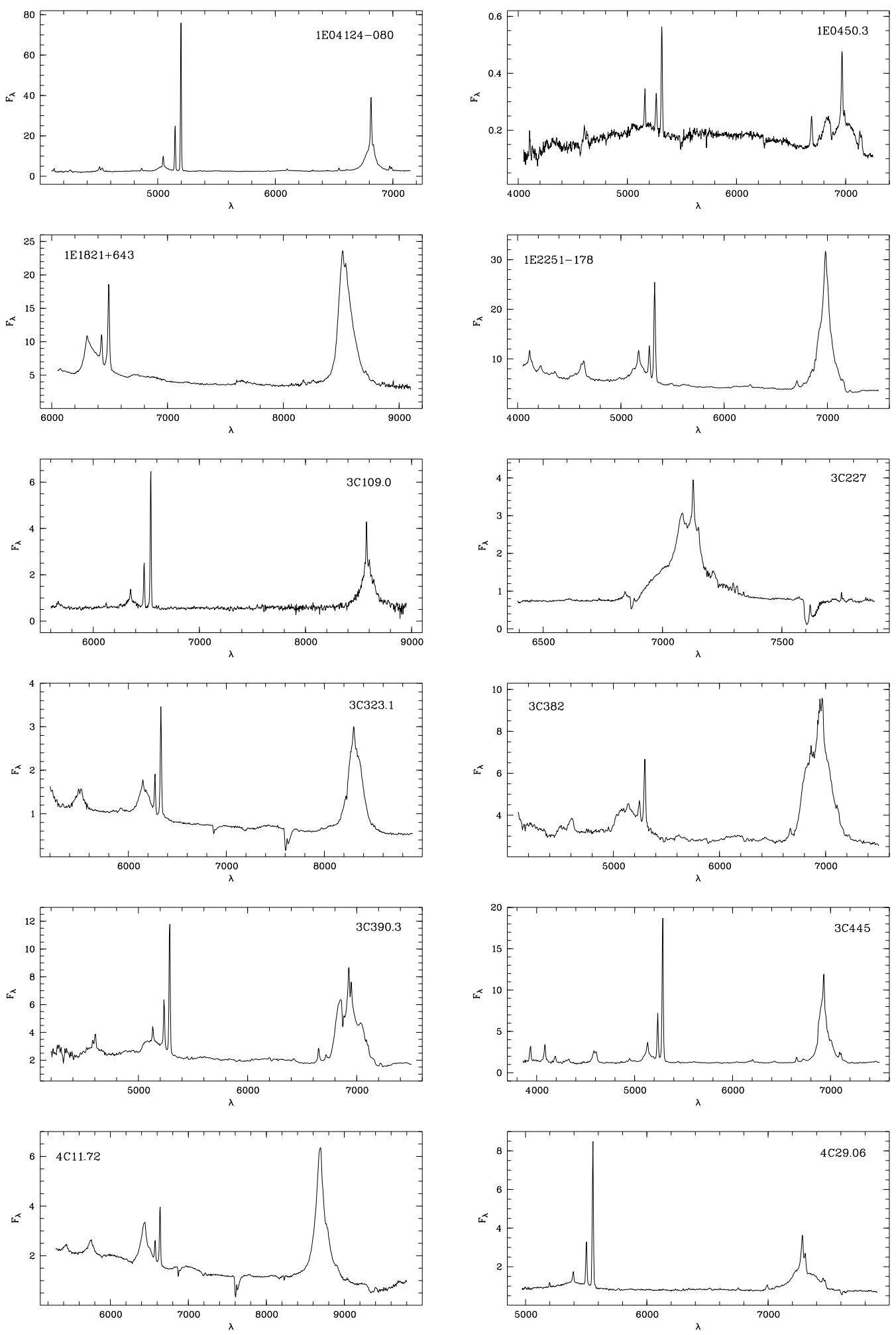

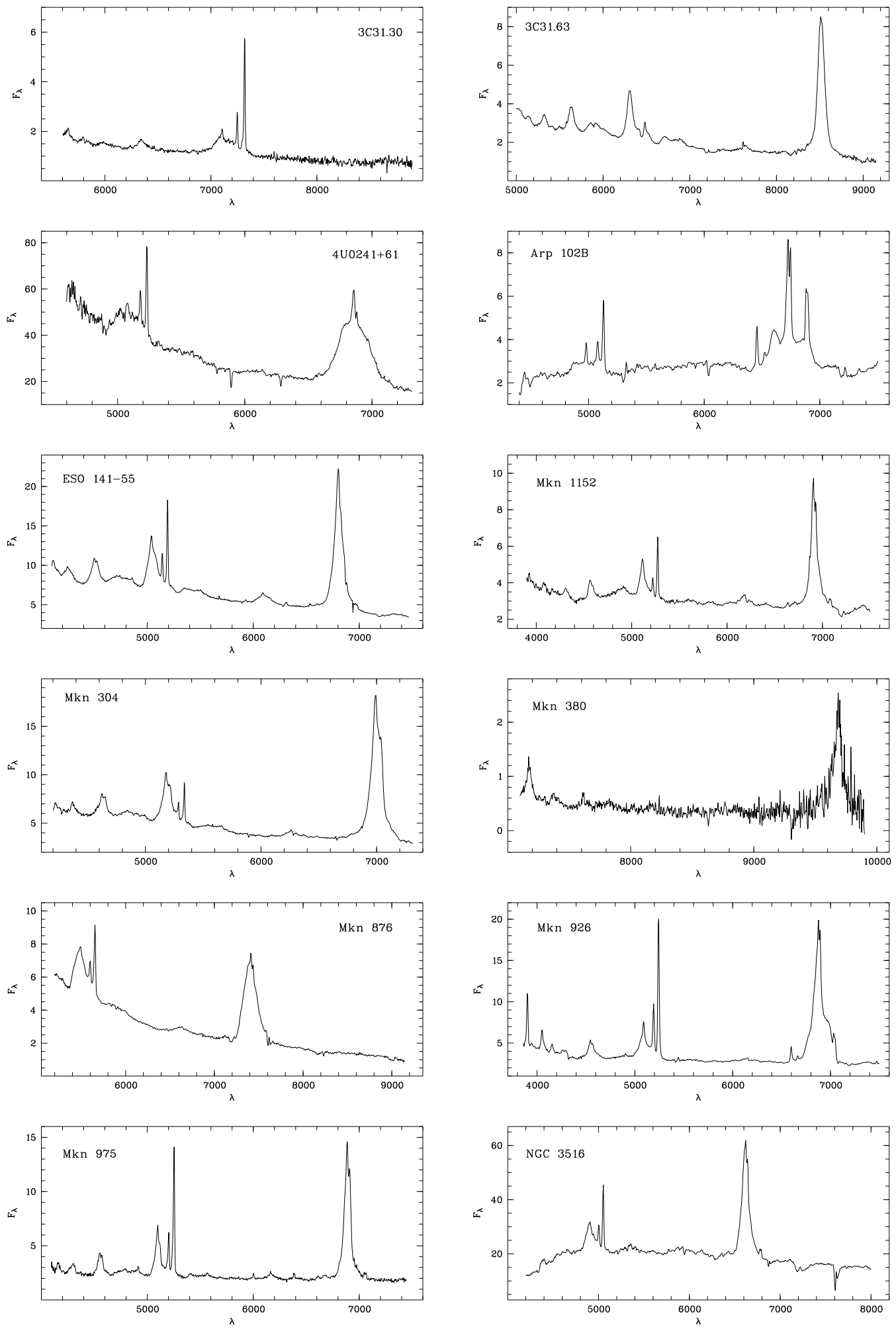

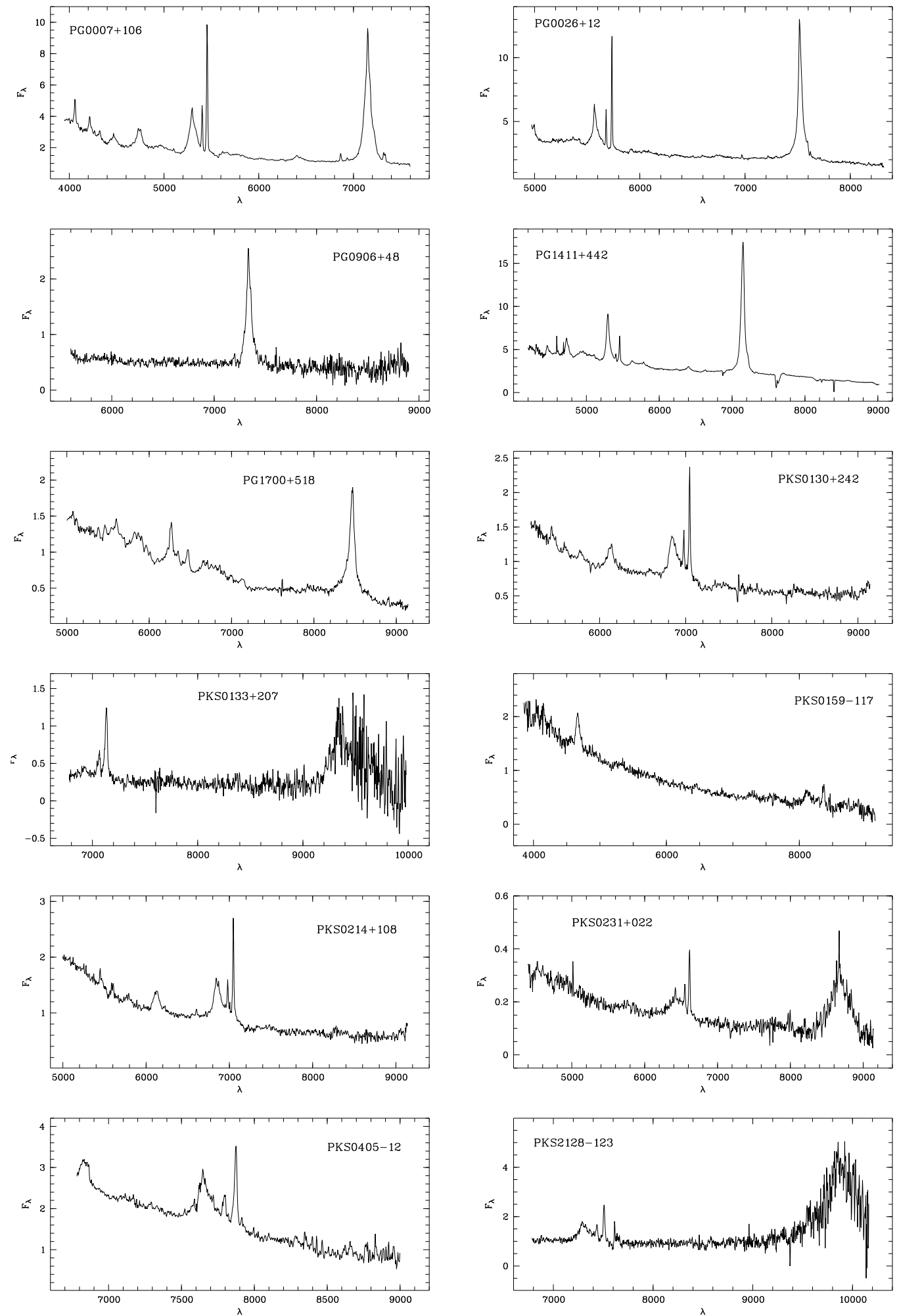

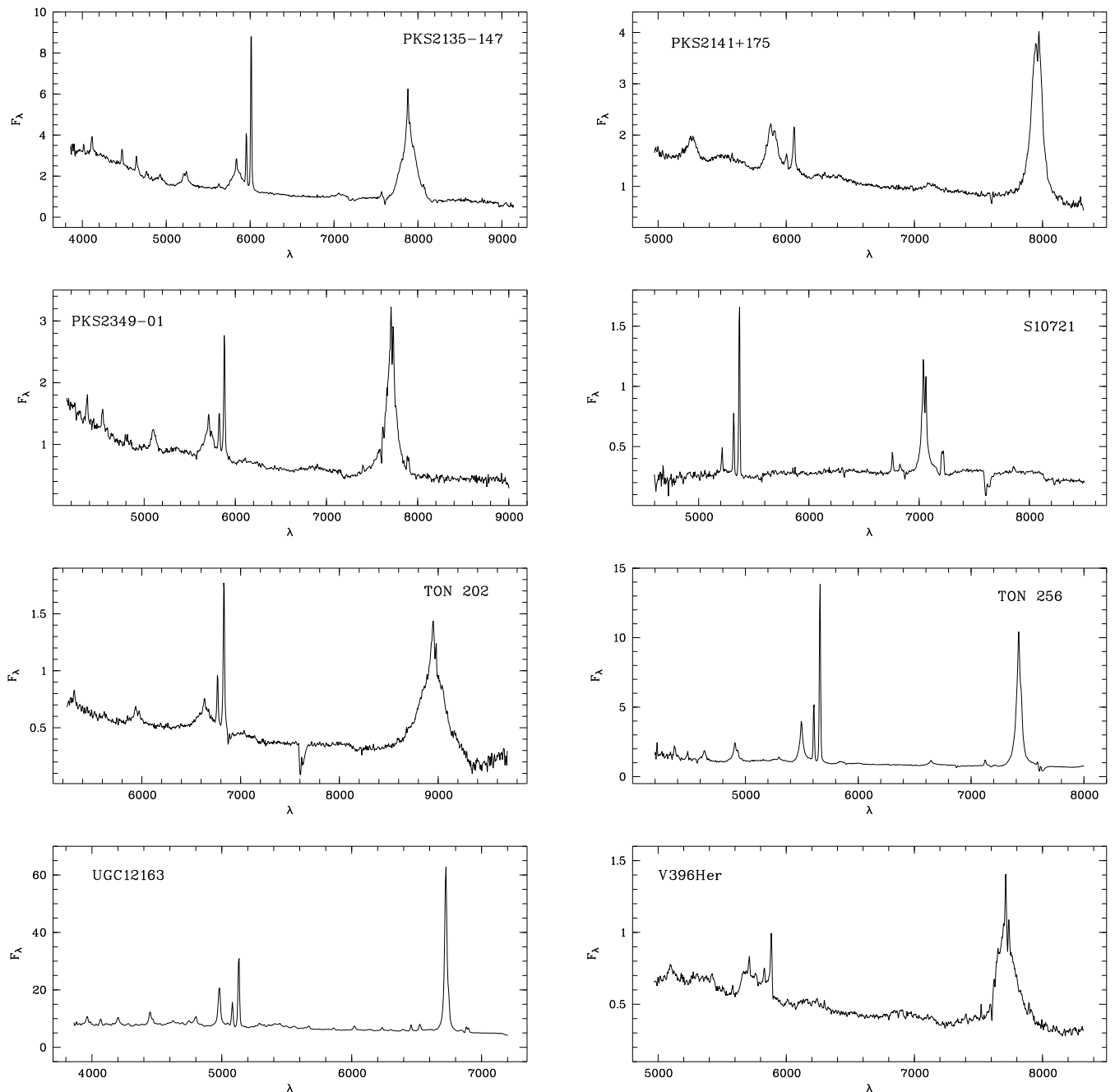

Abbildung 8.1: Optische Spektren der VBLG. Die Beobachtungsdaten dieser Spektren sind in Tabelle 2.3 aufgelistet.
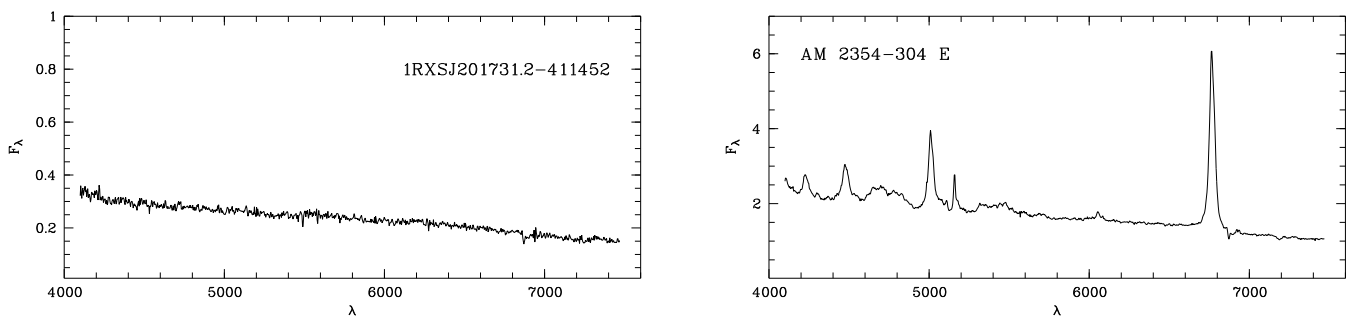

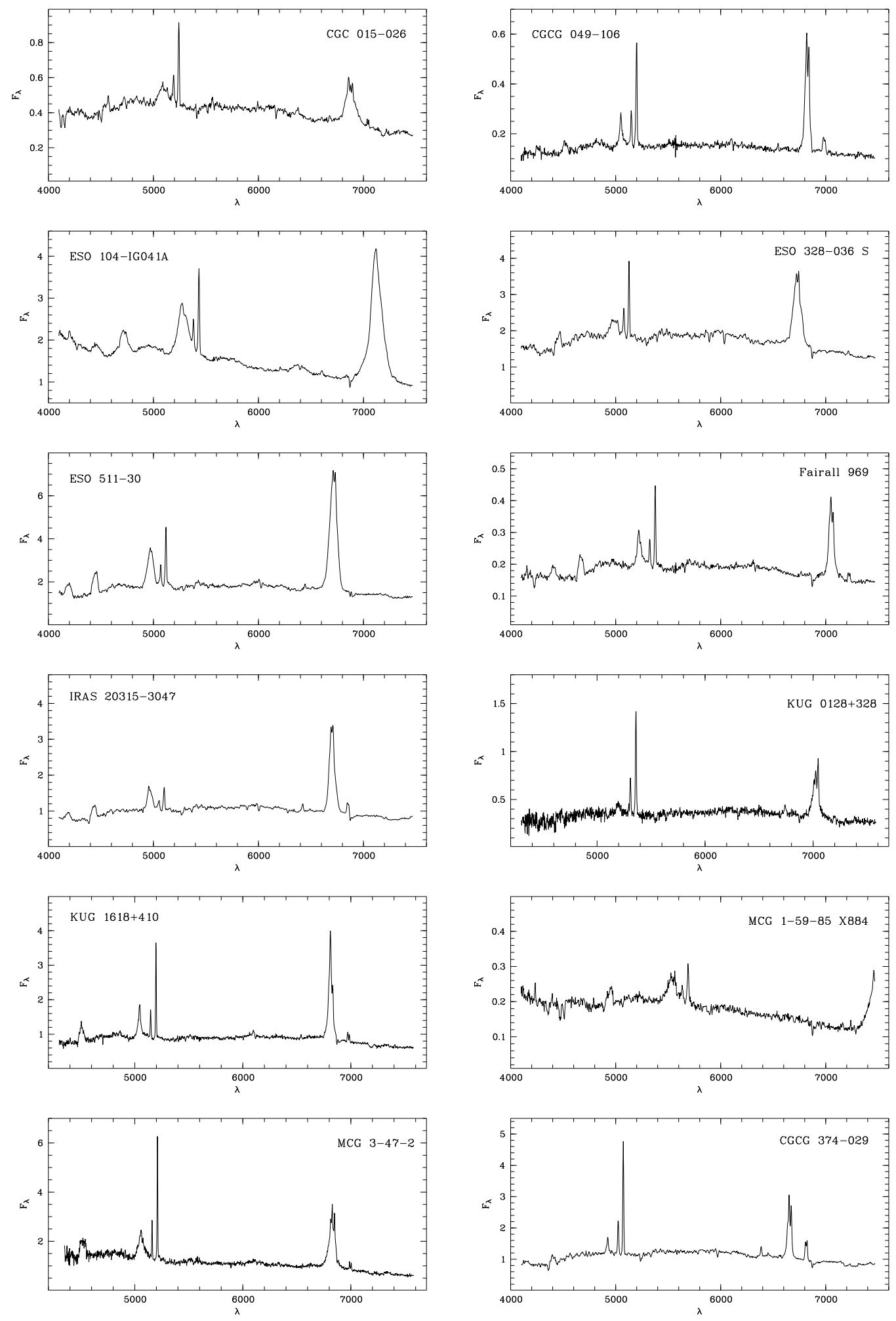

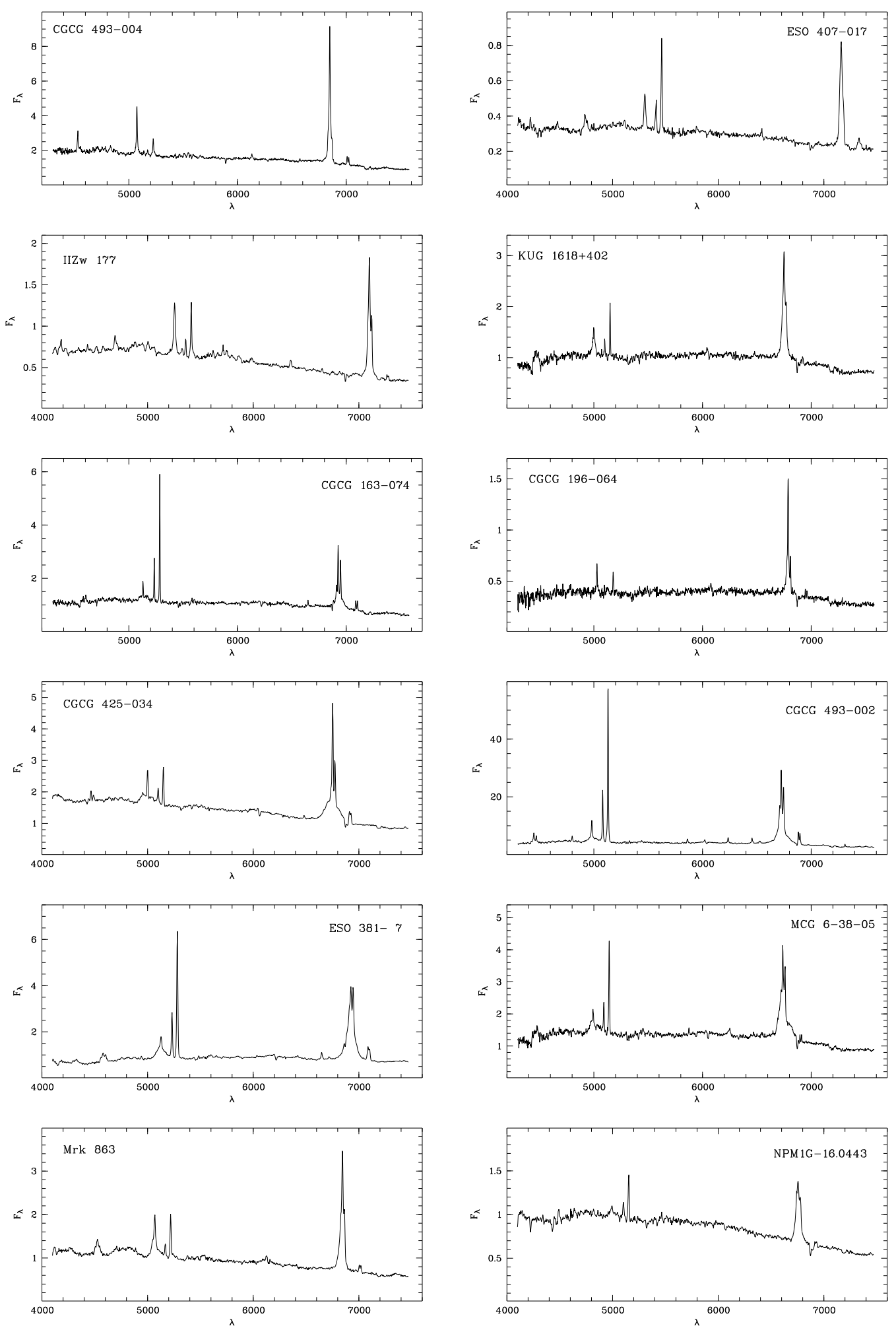

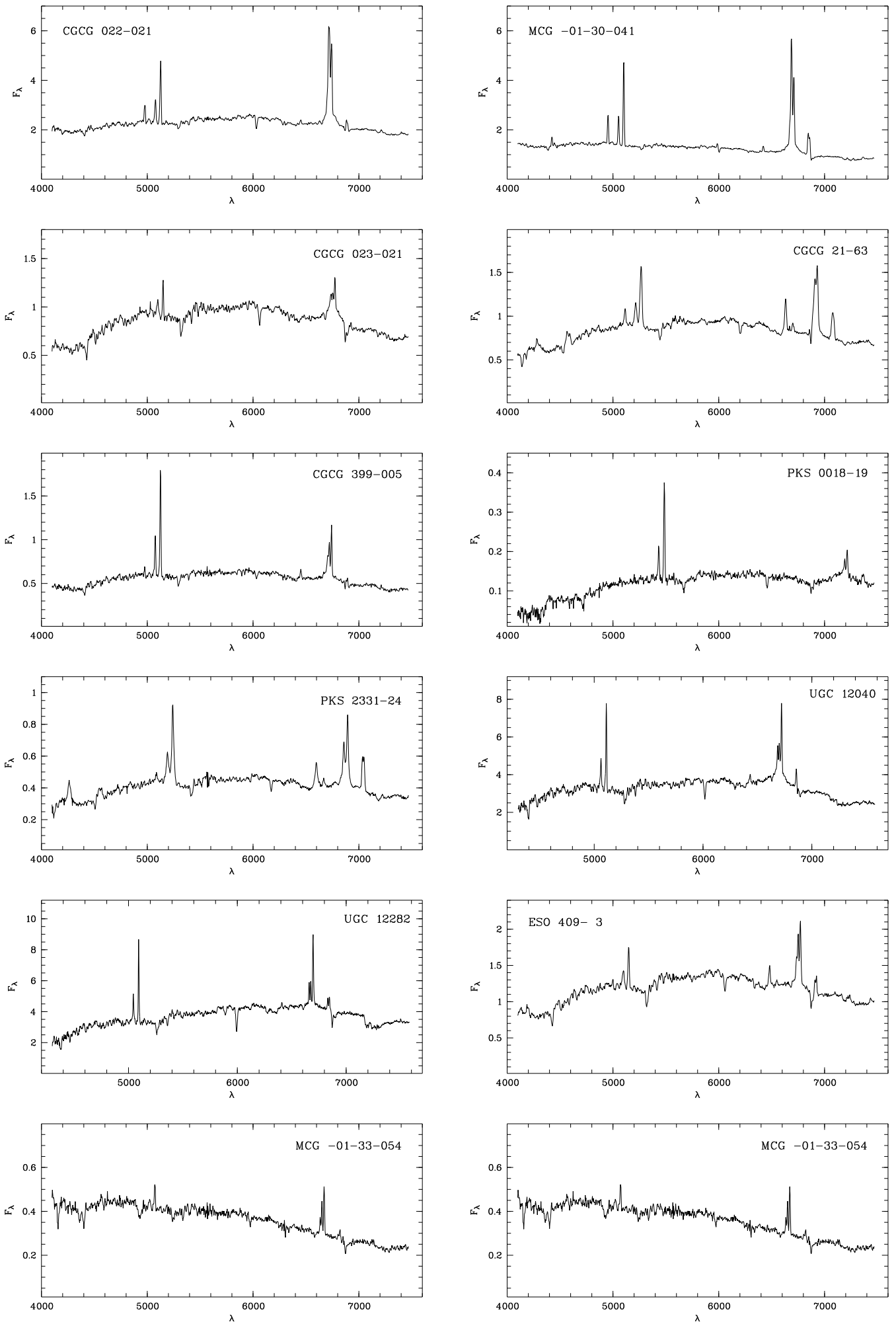

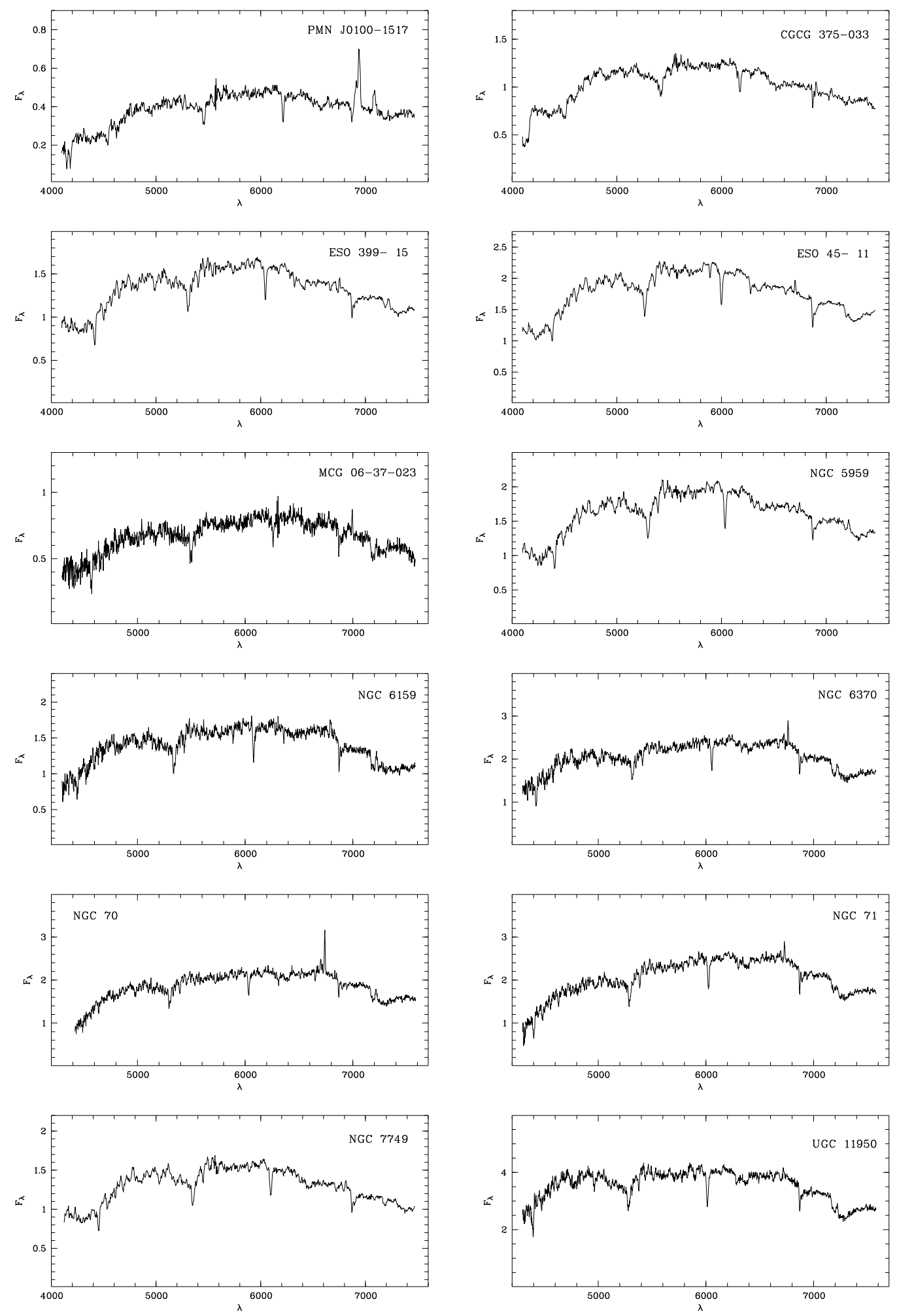

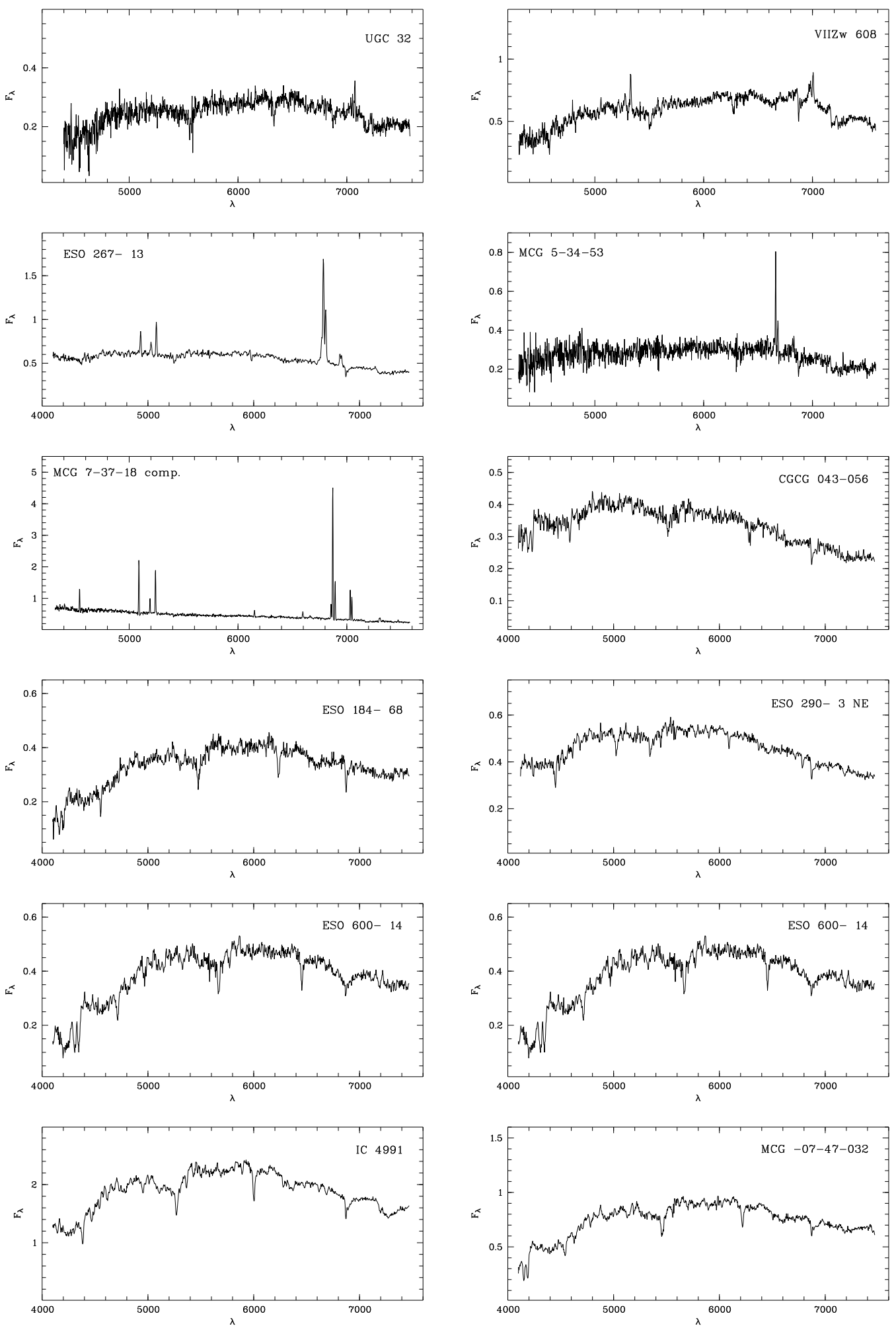

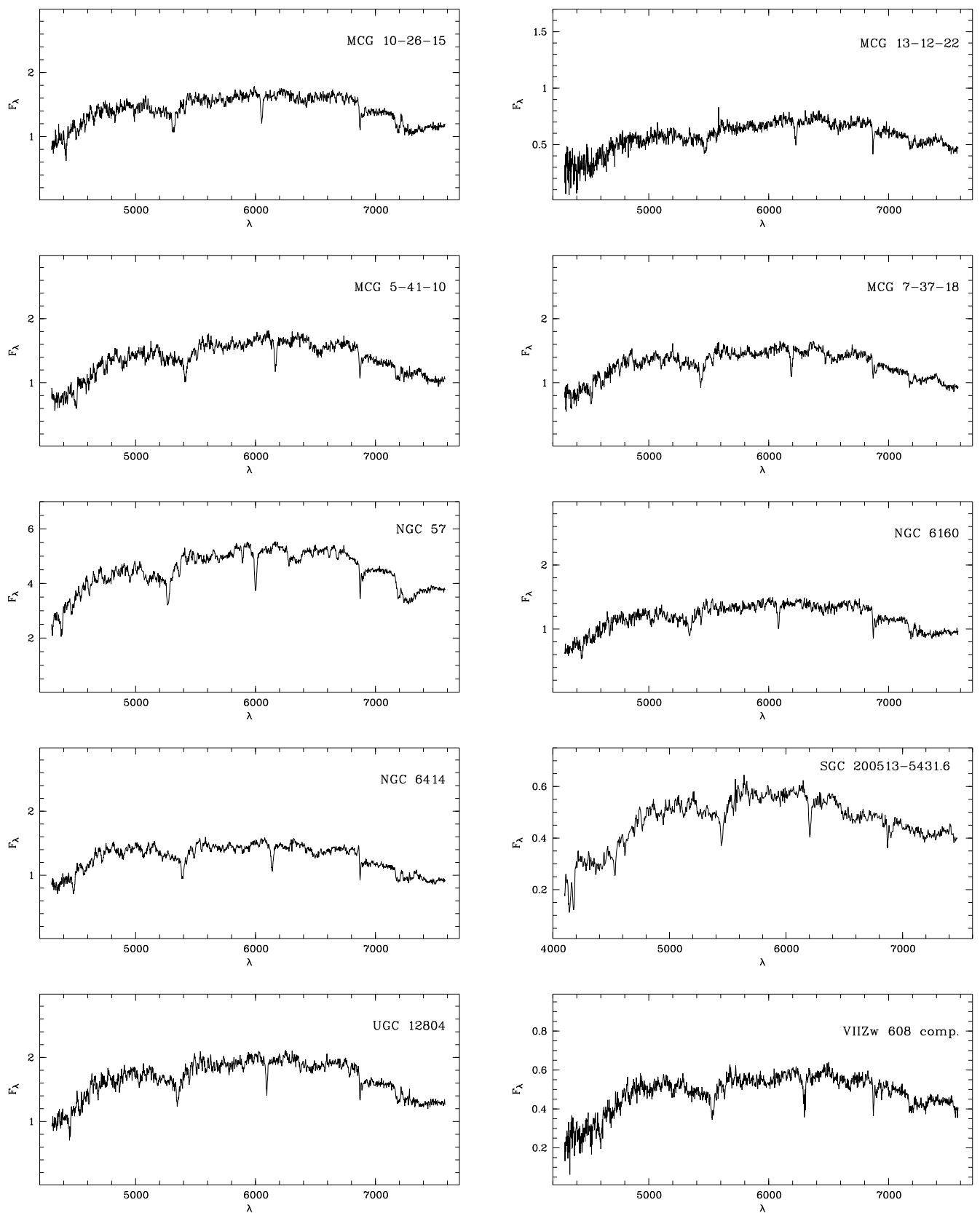

Abbildung 8.2: Optische Spektren der XGAL aus den Beobachtungskampagnen Juli und August 1997; die Spektren der Kampagne November 1996 sind in Pietsch, Bischoff, et al. (1998) abgebildet. Die Beobachtungsdaten der Spektren sind in Tabelle 3.1 aufgelistet. 


\section{Abbildungsverzeichnis}

1.1 Vereinheitlichungs-Schema . . . . . . . . . . . . 8

1.2 Staubscheibe und Jet in $\mathrm{HH}-30 \ldots \ldots$. . . . . . . . . . . . . 12

1.3 Staubtorus im Zentrum von NGC $4261 \ldots$. . . . . . . . . . . . . . 13

2.1 Optisches Spektrum von Arp 102B . . . . . . . . . . . . . . . . . 19

3.1 Linienprofilanalyse am Beispiel von PKS 2349-01 . . . . . . . . . . . 33

3.2 Klassifikation nach Baldwin . . . . . . . . . . . . . 36

3.3 Typische Spektren verschiedener Galaxientypen . . . . . . . . . . . . . 38

3.4 Häufigkeitsverteilung der Galaxien-Typen . . . . . . . . . . . . . . 48

3.5 Absorptionslinienspektrum der Galaxie MCG +07-37-018 . . . . . . . 49

3.6 Verteilung der Rotverschiebungen der Quellen . . . . . . . . . . . . . 51

3.7 Räumliche Verteilung der XGAL . . . . . . . . . . . . . . . . 53

4.1 Datensatz der optische Absoluthelligkeiten . . . . . . . . . . . . . 59

4.2 Verteilung der optischen Absoluthelligkeiten . . . . . . . . . . . . 60

4.3 Optische Absoluthelligkeit als Funktion der Rotverschiebung . . . . . . 61

4.4 Der Röntgendatensatz . . . . . . . . . . . . . . . . . . . . . . . . . . . 64

4.5 Verteilung der Röntgenleuchtkräfte . . . . . . . . . . . . . . . . . 65

4.6 Röntgenleuchtkraft als Funktion der Rotverschiebung . . . . . . . . . . 66

4.7 Der Radiodatensatz . . . . . . . . . . . . . . . . . . . . 71

4.8 Verteilung der Radioleuchtkräfte . . . . . . . . . . . . . . 72

4.9 Radioleuchtkraft als Funktion der Rotverschiebung . . . . . . . . . . . 74

4.10 Der FIR-Datensatz ～. . . . . . . . . . . . . . . 76

4.11 Verteilung der FIR-Leuchtkräfte . . . . . . . . . . . . . . 77 
4.12 FIR-Leuchtkraft als Funktion der Rotverschiebung . . . . . . . . . . 78

4.13 FIR-Leuchtkraft und FIR-Farbe ～. . . . . . . . . . . . . . 80

4.14 Leuchtkraft als Funktion der Rotverschiebung . . . . . . . . . . . . 81

4.15 Fluss als Funktion der Rotverschiebung . . . . . . . . . . . . 83

5.1 Röntgen- und optische Helligkeiten _ . . . . . . . . . . . . . . 87

5.2 Das Flussverhältnis $\mathrm{f}_{\mathrm{X} / \mathrm{B}} \ldots \ldots \ldots \ldots \ldots$

5.3 Das Flussverhältnis $f_{X / B}$ als Funktion des Aktivitätstyps $\ldots . . . . .90$

5.4 Die Ferninfrarot-Radio-Korrelation . . . . . . . . . . . . . . . . 92

5.5 Das Flussverhältnis $\mathrm{f}_{\mathrm{FIR} / \mathrm{R}} \ldots \ldots \ldots \ldots$

5.6 Das Flussverhältnis $\mathrm{f}_{\mathrm{FIR} / \mathrm{R}}$ als Funktion der FIR-Farbe . . . . . . . . . . 95

5.7 Röntgen- und FIR-Helligkeit . . . . . . . . . . . . . . . . 96

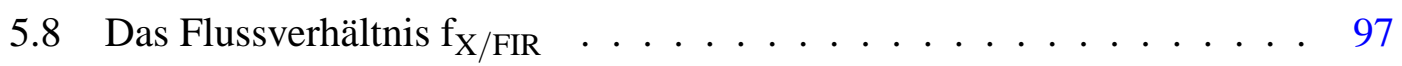

5.9 Das Flussverhältnis $f_{X / F I R}$ als Funktion des Aktivitätstyps . . . . . . . . 99

5.10 FIR- und optische Helligkeiten . . . . . . . . . . . . . . . 100

5.11 Das Flussverhältnis $\mathrm{f}_{\mathrm{FIR} / \mathrm{B}}$ als Funktion der FIR-Leuchtkraft ... 101

5.12 Das Flussverhältnis $\mathrm{f}_{\mathrm{FIR} / \mathrm{B}}$ als Funktion der FIR-Farbe . . . . . . . . 102

5.13 Radio- und optische Helligkeit . . . . . . . . . . . . . . . . . 103

$5.14 \mathrm{f}_{\mathrm{R} / \mathrm{B}}$ zur Unterscheidung von radiolauten/radioleisen Quellen . . . . . 104

5.15 Häufigkeiten von radiolauten und radioleisen Quellen . . . . . . . . . 105

5.16 Mittleres Flussverhältnis $f_{R / B}$ der verschiedenen AGN-Typen . . . . . 107

5.17 Radio- und Röntgenhelligkeit . . . . . . . . . . . . . . . . 108

5.18 Das Flussverhältnis $f_{X / R}$. . . . . . . . . . . . . . . . 109

5.19 Die Flussverhältnisse $\mathrm{f}_{\mathrm{X} / \mathrm{R}}$ und $\mathrm{f}_{\mathrm{R} / \mathrm{B}} \ldots \ldots \ldots \ldots$

5.20 Flussverhältnisse als Funktion der Rotverschiebung . . . . . . . . . . 112

6.1 Emissionslinienbreiten $\mathrm{H} \alpha$ und $\mathrm{H} \beta$. . . . . . . . . . . . . . . 120

6.2 Emissionslinienbreiten . . . . . . . . . . . . . . . 121

6.3 Emissionslinienbreiten im Vergleich zu $\mathrm{M}_{\mathrm{B}}$. . . . . . . . . . . . . 122

6.4 Anteil der AGN-Typen mit breiten Linienkomponenten . . . . . . . . 123

6.5 Verteilung der Linienbreiten . . . . . . . . . . . . . . . 124

6.6 Linienbreiten als Funktion der Rotverschiebung . . . . . . . . . . . 126 
6.7 Leuchtkräfte als Funktion der Linienbreite . . . . . . . . . . . . . . . 129

$6.8 \mathrm{f}_{\mathrm{R} / \mathrm{B}}, \mathrm{f}_{\mathrm{X} / \mathrm{R}}$ und $\mathrm{f}_{\mathrm{FIR} / \mathrm{R}}$ als Funktion der Linienbreite $\ldots \ldots . . . . .131$

6.9 Flussverhältnisse $f_{X / F I R}$ und $f_{\text {FIR/B }}$ als Funktion der Linienbreite . . . . 132

6.10 Flussverhältnis $f_{X / B}$ als Funktion der Linienbreite . . . . . . . . . 133

6.11 Balmerdekrement als Funktion der Linienbreite . . . . . . . . . . . 134

6.12 Balmerdekrement als Funktion der Leuchtkräfte . . . . . . . . . . . . 135

6.13 Balmerdekrement als Funktion der Flussverhältnisse . . . . . . . . 137

7.1 Modell des Zentralbereichs von Mrk $110 \ldots 155$

8.1 Optische Spektren der VBLG . . . . . . . . . . . . . . . . 176

8.2 Optische Spektren der XGAL . . . . . . . . . . . . . . . . . 182 


\section{Tabellenverzeichnis}

1.1 Taxonomisches Schema Aktiver Galaxien . . . . . . . . . . . . 6

2.1 Liste der Beobachtungskampagnen . . . . . . . . . . . . . . . . 23

2.2 Liste der verwendeten CCD-Detektoren . . . . . . . . . . . . . . 24

2.3 Liste der VBLG . . . . . . . . . . . . . . . . . . . . 26

3.1 Liste und Identifikationen der röntgenselektierten Galaxien XGAL . . 40

3.2 Diagnostische Linienverhältnisse . . . . . . . . . . . . . . . . . . . 44

3.3 Häufigkeiten der verschiedenen AGN-Typen . . . . . . . . . . . . . . . 47

3.4 Statistik der Rotverschiebungen . . . . . . . . . . . . . . 52

4.1 Statistik der absoluten optischen Helligkeiten . . . . . . . . . . . . . . 59

4.2 Statistik der Röntgenleuchtkräfte . . . . . . . . . . . . . . . . . 65

4.3 Radiodurchmusterungen . . . . . . . . . . . . . . . . . . 67

4.4 Die PMN Radiodurchmusterung . . . . . . . . . . . . . . . . . 68

4.5 Quellen der Radiodaten . . . . . . . . . . . . . . . . . . . . . . . 70

4.6 Statistik der Radioleuchtkräfte . . . . . . . . . . . . . . . . . . . 73

4.7 Statistik der FIR-Leuchtkräfte . . . . . . . . . . . . . . . . . . 77

5.1 Statistik des Flussverhältnis $\mathrm{f}_{\mathrm{X} / \mathrm{B}} \ldots \ldots \ldots$

5.2 Statistik des Flussverhältnis $\mathrm{f}_{\mathrm{FIR} / \mathrm{R}} \ldots \ldots$. . . . . . . . . . . 94

5.3 Statistik des Flussverhältnis $\mathrm{f}_{\mathrm{X} / \mathrm{FIR}} \ldots \ldots$. . . . . . . . . . 98

5.4 Lineare Regression und Korrelationsanalyse von $\mathrm{f}_{\mathrm{FIR} / \mathrm{B}}$ und $\mathrm{L}_{\mathrm{FIR}}$. . . . 101

5.5 Häufigkeiten von radioleisen und radiolauten Quellen . . . . . . . . . 106

5.6 Korrelationen der Flussverhältnisse mit der Rotverschiebung . . . . . . 111 
5.7 Korrelationsanalyse der Flüsse und der Leuchtkräfte . . . . . . . . . . . 114

5.8 Korrelationsanalyse der Flussverhältnisse mit den Leuchtkräften . . . 115

6.1 Statistik der Balmer-Linienbreiten . . . . . . . . . . . . . . 125

6.2 Korrelation der Linienbreite mit der Rotverschiebung . . . . . . . . . . 126

6.3 Statistik der Leuchtkräfte . . . . . . . . . . . . . . . . . . . . . . . . 139

6.4 Korrelationen mit der Linienbreite . . . . . . . . . . . . . . . . . . 140

6.5 Statistik der Flussverhältnisse . . . . . . . . . . . . . . . . . . . . 141

6.6 Statistik des Balmerdekrements . . . . . . . . . . . . . . . . . . . . . 142

6.7 Korrelationen des Balmerdekrements . . . . . . . . . . . . . . . . . . . 142

7.1 Häufigkeiten der verschiedenen XGAL-Typen . . . . . . . . . . . . . . 144

8.1 Multifrequenzdaten . . . . . . . . . . . . . . . 160

8.2 Ergebnisse der optischen Spektroskopie . . . . . . . . . . . . . . 164

8.3 Halbwertsbreiten aus Zweikomponenten-Messungen . . . . . . . . . . 168

8.4 Neutralgasdichte und Röntgenzählrate . . . . . . . . . . . . . . . 169 


\section{Literaturverzeichnis}

Abazajian, K., et al. 2003, AJ, 126, 2081

Alef, W., Wu, S.Y., Preuss, E., Kellerman, K.I., \& Qiu, Y.H. 1996, A\&A, 308, 376

Allen, C.W., 1976, Astrophysical Quantities, 3rd Ed.

Antonucci, R. 1982, Nature, 299, 605

Antonucci, R. 1993, ARA\&A, 31, 473

Awaki, H., Terashima, Y., Hayashida, K., \& Sakano, M. 2001, PASJ, 53, 647

Baldwin, J.A., Phillips, M.M., Terlevich, R, 1981, PASP 93, 5

Barth, A.J., Filippenko, A.V., \& Moran, E.C. 1999, ApJ, 515, 61

Barth, A.J., Filippenko, A.V., \& Moran, E.C. 1999, ApJ, 525, 673

Barth, A.J. 2002, Low-Luminosity AGNs and Unification. In: Maiolino, R., Marconi, A., \& Nagar, N. (eds.) Issues in Unification of Active Galactic Nuclei, ASP Conference Proceedings, Vol. 258, p. 147

Barthel, P.D. 1989, ApJ, 336, 606

Becker, R.H., White, R.L., \& Edwards, A.L. 1991, ApJS, 75

Becker, R.H., White, R.L., \& Helfand, D.J. 1995, ApJ, 450, 559

Beichman, C., Neugebauer, G., Habing, H.J., Clegg, P.E., and Chester, T.J. (eds.), 1988, IRAS Catalogs and Atlases Explanatory Supplement, NASA RP-1190 (Washington, DC: GPO), vol 1

Beuing, J., Döbereiner, S., Böhringer, H., \& Bender, R. 1999, MNRAS, 302, 209

Biermann, P. 1976, A\&A, 53, 295 
Birkinshaw, M., \& Davies, R.L. 1985, ApJ, 291, 32

Bischoff, K., 1993, Langzeitvariabilitätsanalyse morphologisch pekuliärer Seyfert-1 Galaxien, Diplomarbeit, Universität Göttingen

Bischoff, K., \& Kollatschny, W. 1994, AGAb, 10, 235

Bischoff, K., Kollatschny, W., Pietsch, W. 1995, AGAb, 11, 218

Bischoff, K., Kollatschny, W., Pietsch, W. 1996, HRI Observations of Selected Seyfert Galaxies. In: Zimmermann, H.U., Trümper, J.E., \& Yorke H. (eds.), Röntgenstrahlung from the Universe, p. 423

Bischoff, K., Kollatschny, W., Dietrich M. 1997, Long-Term Variability of AGN. In: Peterson, B.M., Cheng, F.-Z., \& Wilson A.S. (eds.), Emission Lines in Active Galaxies: New Methods and Techniques, p. 171

Bischoff, K., Kollatschny, W., Pietsch, W., 1998, AGM, 14, 138

Bischoff, K., Kollatschny, W., 1999, A\&A, 345, 49

Bischoff, K., Pietsch, W., Boller, T., Döbereiner, S., Kollatschny, W., Zimmermann, H.U., 1999, Properties of new X-ray selected AGN. In: Aschenbach B., Freyberg M.J. (eds.) Highlights in X-ray Astronomy, MPE Report 272, p. 226

Bischoff, K., 2003, Properties of Optical and X-ray Selected AGNs - Probing the Unified Model of AGNs. In: Komossa, S., et al. (eds.) Proc. of the China-Germany workshop on the Multiwavelength View on Active Galactic Nuclei, Lijiang 2002, in press

Blandford, R.D., \& Rees, M. 1974, MNRAS, 169, 395

Blandford, R.D., \& Rees, M. 1978, In: Wolfe, A.M. (ed.), Pittsburgh Conference on BL Lac Objects, p. 328

Blandford, R.D., \& Payne, D.G. 1982, MNRAS, 199, 883

Blandford, R.D., \& Begelman, M.C. 1999, MNRAS, 302, L1

Boller, T., Bertoldi, F., 1996, X-Ray Loud LINERs-Starburst vs. AGN Activity. In: Eracleous, M., Koratkar, A., Leitherer, C., Ho, L. (eds.), The Physics of Liners in view of recent observations. ASP Conference Series 103, p.159

Boller, T., 1999, Ap\&SS, 266, 49

Boulanger, F., Beichman, C., Desert, F.X., Helou, G., Perault, M., Ryter, C., 1988, ApJ 332,328 
Brinkmann, W., Siebert, J., Reich, W., et al., 1995, A\&AS 109, 147

Brotherton, M.S. 1996, ApJS, 102, 1

Burrows, C.J., Stapelfeldt, K., Watson, A.M., et al. 1996, ApJ 473, 437

Burstein, D., \& Heiles, C. 1982, AJ, 87, 1165

Burstein, D., \& Heiles, C. 1984, ApJS, 54, 33

Chambers, K..C., Miley, G.K., \& van Breugel, W. 1987, Nature, 329, 605

Chen, K., Halpern, J.P., Filippenko, A.V., 1989, ApJ, 339, 742

Chiang, J., \& Murray, N. 1996, ApJ, 466, 704

Cohen, M.H., Ogle, P.M., Tran, H.D., et al. 1999, AJ, 118, 1963

Collin-Souffrin, S., Dumont, A.M., 1989, A\&A 213, 39

Collin, S. 2001, Accretion and Emission Processes in AGN. In: Aretxaga, I., Kunth, D., \& Mújica (eds.) Advanced Lectures on the Starburst-AGN Connection, Proceedings of a conference held in Tonantzintla, Puebla, Mexico, World Scientific, Singapore, 2001, p.167

Condon, J.J., Cotton, W. D., Greisen, E. W., et al. 1998, AJ 115, 1693

Cox, P., Krügel, E., Mezger, P.G., 1986, A\&A 155, 380

de Jong, T., Clegg, P.E., Soifer, B.T., et al., 1984, ApJ 278, L67

de Jong, T., Klein, U., Wielebinski, R., Wunderlich, E., 1985, A\&A 147, L6

Dennett-Thorpe, J., Barthel, P.D., van Bemmel, I.M., 2000, A\&A, 364, 501

Dickey, J.M., Lockman, F.J., 1990, ARA\&A 28, 215

DierckxxP. 1982, Computer Graphics and Image Processing, vol. 20

Dietrich, M., Bischoff, K., \& Kollatschny, W., 1993, AGAb, 9, 184

Dietrich, M., Peterson, B. M., Albrecht, P., et al. 1998, ApJS, 115, 185

D’Odorico, S., Gross, W.M., \& Dopita M.A. 1982, MNRAS, 198, 1059

Dopita, M., 1997, PASA 14, 230

Dopita, M., 2000, ApSS 272, 79 
Draine, B.T., Lee, H.M., 1984, ApJ 285, 89

Emmering, R.T., Blandford, R.D., \& Shlosman, I. 1993, ApJ, 385, 460

Eracleous M., Halpern J.P. 1993, ApJS, 90, 1

Eracleous, M., Halpern, J., 2003, ApJ 590, in press

Erkens, U., Wagner, S.J., Alloin, D., et al. 1995, A\&A, 296, 90

ESO Users Manual, 1983, Garching

Fabbiano, G., Kim, G.-W., \& Trinchieri, G. 1992, ApJS 80, 531

Falcke, H., Sherwood, W., Patnaik, A.R., 1996, ApJ 473, L13

Fanaroff, B.L., \& Riley, J.M. 1974, MNRAS, 167, 31

Ferrarese, L., Ford, H.C., \& Jaffe, W. 1996, ApJ, 470, 444

Filippenko. A.V., 1996, The Physics of LINERs in View of Recent Observations. In: Eracleous, M., Koratkar, A., Leitherer, C., Ho, L. (eds.), ASP Conf. Ser. Vol. 103, 17

Filippenko, A.V. 2003, LINERs and their Physical Mechanisms. In: Collin, S., Combes, F., \& Shlosman, I. (eds.) Active Galactic Nuclei: from Central Engine to Host Galaxy, ASP Conference Series, Vol. 290, p. 369

Gaskell, C.M. 1996, ApJ, 464, 107

Ghisellini, G., Padovani, P., Celotti, A., \& Maraschi, L. 1993, ApJ, 407, 65

Gonzalez, A.H. \& Faber, S.M. 1997, ApJ, 485, 80

Gregory, P.C., \& Condon, J.J. 1991, ApJS 75, 1011

Griffith, M.R., \& Wright, A.E. 1993, AJ 105, 1666

Griffith, M.R., Wright, A.E., Burke, B.F., Ekers R.D. 1994, ApJS 90, 179

Griffith, M.R., Wright, A.E., Burke, B.F., Ekers R.D. 1995, ApJS 97, 347

Gondhalekar, P.M., Rouillon-Folley, C., \& Kellet B.J. 1997, MNRAS, 288, 260

Goodrich, R.W., 1989, ApJ 342, 224

Halpern, J.P., 1990, ApJ, 365, L51 
Hardie, R.H. 1962, Photoelectric Reductions. In: Hiltner, W.A. (ed.) Stars and Stellar Systems, Vol. II: Astronomical Techniques, The University Press of Chicago, Chicago, p. 178

Hasinger, G., Trümper, J., Schmidt, M., 1991, A\&A, 246, L2

Heckman, T.M., 1980, A\&A, 87, 152

Heisler, C.A., Lumsden, S.L., \& Bailey, J.A. 1997, Nature, 385, 700

Heisler, C.A., 1999, Investigating the Nuclei of $60 \mu \mathrm{m}$ Peaker Galaxies. In: Gaskell, C.M., et al. (eds.), Structure and Kinematics of Quasar Broad Line Regions, ASP Conference Series, Vol. 175, Lincoln Proc., p. 405

Helou, G., Soifer, B.T., Rowan-Robinson, M., 1985, ApJ, 298, L7

Helou, G., Khan, I.R., Malek, L., Boehmer, L., 1988, ApJS 68, 151

Ho, L.C., Filippenko, A.V. \& Sargent, W.L.W. 1997a, ApJ, 487, 568

Ho, L., Filippenko, A., Sargent, W., Peng, C., 1997b, ApJS 112, 391

Ho, L., Filippenko, A., Sargent, W., Peng, C., 1997c, ApJS 112, 315

Ho, L.C., Peng, C.Y., 2001, ApJ 555, 650

Ho, L.C. 2002, Nonstandard Central Engines in Nearby Galaxies. In: Maiolino, R., Marconi, A., \& Nagar, N. (eds.) Issues in Unification of Active Galactic Nuclei, ASP Conference Proceedings, Vol. 258

Ho, L.C. 2003, The Central Engine of Low-Luminosity AGN. In: Collin, S., Combes, F., \& Shlosman, I. (eds.) Active Galactic Nuclei: from Central Engine to Host Galaxy, ASP Conference Series, Vol. 290, p. 379

Horne, K. 1986, PASP, 98, 609

Hough, D.H., \& Readhead, A.C.S. 1989, AJ, 98, 1208

IRAS Catalog of Point Sources, 1986, Joint IRAS Science Working Group

Iyengar, K.V.K, Rengarajan, T.N., Verma, R.P., 1986, The relationsship between Blue and FIR luminosities of spiral galaxies. In: Israel F.P. (ed.) Light on Dark Matter, Reidel Publishing Company

Jaffe, W., Ford, H., Ferrarese, L., van den Bosch, F., \& O’Connell, R.W. 1996, ApJ, 460, 214 
Jones, D.L., Wehrle, A.E., Meier, D.L., \& Piner, B.G. 2000, ApJ, 534, 165

Kartje, J.F., Königl, A., Hwang, C.-Y., Bowyer, S. 1997, ApJ, 474, 630

Keel, W.C., 1993, AJ 106, 1771

Kellermann, K.I., Sramek, R., Schmidt, M., Shaffer, D.B., Green, R., 1989, AJ, 98, 1195

Kembhavi, A., Feigelson, E.D., Singh, K.P., 1986, MNRAS 220, 51

Khachikian, E.E., Weedman, D.W., 1971, Afz 7

Kotilainen, J.K., Falomo, R., \& Scarpa, R. 1998, A\&A, 336, 479

Komossa, S., Böhringer, H., \& Huchra, J.P., 1999, A\&A, 349, 88

Komossa, S., Breitschwerdt, D., Böhringer, H., \& Meerschweinchen, J. 2000, Ap\&SS, 272,295

Klaas, U., 1988, Dissertation, Ruprecht-Karls-Universität Heidelberg

Klöckner, H.-R., Baan, W.A., \& Garrett, M.A. 2003, Nature, 421, 821

Kollatschny, W., \& Bischoff, K. 1999, Profile variations in AGN spectra. In: Gaskell, C.M., Brandt, W.N., Dietrich, M., Dultzin-Hacyan, D., \& Eracleous, M. (eds.) Structure and Kinematics of Quasar Broad Line Regions, ASP Conf. Series 175, p. 61

Kollatschny, W., Bischoff, K., \& Dietrich, M., 2000, A\&A, 361, 901

Kollatschny, W., Bischoff, K., Robinson, E.L., Welsh, W.F., \& Hill, G.J. 2001, A\&A 379,125

Kollatschny, W., \& Bischoff, K., 2002, A\&A, 386, L19

Kollatschny, W. 2003, A\&A, 412, L61

Königl, A., \& Kartje, J.F. 1994, ApJ, 434, 446

Kuhr, H., Witzel, A., Pauliny-Toth, I.I.K., \& Nauber, U. 1981, A\&AS, 45, 376

Laor, A., 2000, ApJ 543, L111

Laor, A., Fiore, F., Wilkes, B.J., \& McDowell, J.C. 1997, ApJ, 477, 93

Lehnert, M.D., Heckman, T.M., 1995, ApJS 97, 89

Maccacaro, T., Gioia, I.M., Wolter, A., Zamorani, G., Stocke, J.T. 1988, ApJ, 326, 680 
Malkan, M.A., Gorijan, V., \& Tam, R. 1998, ApJS, 117, 25

Malmquist, K.G. 1920, Medd. Lund Astron. Obs., Ser. II, 22, 1

Malmquist, K.G. 1924, Medd. Lund Astron. Obs., Ser. II, 32, 64

Markarian, B. E. 1967, Afz, 3, 55

Marsh, T.R. 1988, MN, 231, 1117

Matsushita, K., Ohashi, T., \& Makishima, K. 2000, PASJ, 52, 685

Mazzarella, J.M. \& Balzano, V.A. 1986, ApJS, 62, 751

Matthews, T.A., Morgan, W.W., \& Schmidt, M. 1964, ApJ, 140, 35

Malizia, A., Bassani, L., Stephen, J.B., Malaguti, G., \& Palumbo, G.G.C. 1997, ApJS, 113,311

Mc Carthy, P.J., van Breugel, W., Spinrad, H., \& Djogovski, S., 1987, ApJ, 321, 29

McLure, R.J., Kukula, M.J., Dunlop, J.S., et al., 1999, MNRAS 308, 377

McLure, R.J., Dunlop, J.S., 2001, MNRAS 321, 515

McLure, R.J., Dunlop, J.S., 2001, The radio loudness dichotomy: environment or blackhole mass? In: Márquez, I., et al. (eds.), QSO hosts and their environments, Kluwer Academic Publishers, p.27

Meisenheimer, K., Haas, M., Müller, S.A.H., Chini, R., Klaas, U., \& Lemke, D. 2001, A\&A, 372719

Miller, J.S., \& Antonucci, R.R.J., 1983, ApJ, 271, L7

Miller, J.S., Peterson, B.M., 1990, ApJ 361, 98

Miller, P., Rawlings, S., Saunders, R., 1993, MNRAS 263, 425

Murray, N., \& Chiang, J. 1997, ApJ, 474, 91

Murray, N., \& Chiang, J. 1998, ApJ, 494, 125

Neugebauer, G., Habing H.J., van Duinen R., et al. 1984, ApJ, 278, L1

Nicklas S., 1997, A\&A 322, 29: survey 2.8cm

Oke, J.B. 1990, AJ 99, 1621 
Orr, M.J.L., \& Browne, I.W.A. 1982, MNRAS, 200, 1067

Oshlack, A.Y.K.N., Webster, R.L., \& Whiting, M.T. 2002, ApJ, 576, 810

Osmer, P.S. The Evolution of Quasars. In: Ho, L.C. (ed.) Coevolution of Black Holes and Galaxies, Carnegie Observatories Astrophysics Series, Vol. 1, Cambridge Univ. Press

Osterbrock, D.E., 1977, ApJ 215, 733

Osterbrock, D.E., 1989, Astrophysics of Gaseous Nebulae and Active Galactic Nuclei (University Science Books)

Osterbrock, D.E., Pogge R.W., 1985, ApJ 297, 166

O’Sullivan, E., Duncan, A.F., \& Ponman, T.J. 2001, MNRAS, 328, 461

Paturel, G., Fouque, P., Bottinelli, L., Gouguenheim, L., 1989, A\&AS, 80, 299

Percival, W.J., Miller, L., McLure, R.J., Dunlop, J.S., MNRAS 322, 843

Perez, E., Robinson, A., \& de la Fuente, L. 1992a, MNRAS 255, 502

Perez, E., Robinson, A., \& de la Fuente, L., 1992b, MNRAS 256, 103

Perley, R.A., Dreher, J.W., \& Cowan, J.J. 1984, ApJ, 285, L35

Peterson, B.M., 1988, PASP, 100, 18

Peterson, B.M., 1997, An Indroduction to Active Galactic Nuclei, Cambridge University Press, Cambridge

Peterson, B.M., Wandel, A., 1999, AAS 194, 4912

Peterson, B.M., Wandel, A., 2000, ApJ 540, 13

Pietsch, W., Bischoff, K., Boller, T., Döbereiner, S., Kollatschny, W., Zimmermann, H.U., 1998, A\&A, 333, 48

Quataert, E., di Matteo, T., Narayan, R., \& Ho, L.C. 1999, ApJ, 525, 89

Quataert, E. 2001, Low Radiative-Efficiency Accretion Flows. In: Peterson, B.M., Pogge, R.W., \& Polidan, R.S. (eds.) Probing the Physics of Active Galactic Nuclei, ASP Conference Proceedings, Vol. 224., p.71

Reich, W., Fürst, E., Reich, P., Kothes, R., Brinkmann, W., Siebert, J., 2000, A\&A 363, 141 
Rokaki, E., Boisson, C., Collin-Souffrin, S. 1992, A\&A 253, 57

ROSAT Consortium, 1999, ROSAT NEWS No. 69

Rowan-Robinson, M., 1987, Infrared properties of IRAS galaxies. In: Thuan, T.X, et al. (eds.), Starbursts and galaxy evolution, Editions Frontieres 1987, p. 235-255.

Sambruna, R.M., Eracleous, M., \& Mushotzky, R.F. 1999, ApJ, 526, 60

Sandage, A. 1973, ApJ, 180, 687

Savage, B.D., \& Mathis, J.S. 1979, ARA\&A, 17, 73

Seyfert, C.K. 1943, AJ, 97, 28

Shields, G.A. 1999, PASP, 111, 661

Shields, G.A., Rix, H.-W., McIntosh, D.H., et al. 2001, Variability in LINERs. In: Peterson, B.M., Pogge, R.W., \& Polidan, R.S. (eds.) Probing the Physics of Active Galactic Nuclei, ASP Conference Proceedings, Vol. 224., p.327

Smith, D.A, \& Done, C. 1996, MNRAS, 280, 355

Soifer, B.T., Neugebauer, G., Houck, J.R., 1987, ARA\&A, 25, 187

Stocke, J.T., Morris, S.L., Gioia, I.M., et al. 1991, ApJS 76, 813

Stoughton, D., Lupton, R.H., Bernardi M., et al. AJ, 123, 485

Strateva, I.V., Strauss, M.A., Hao, L., 2002, Double-Peaked Optical Emission Lines and the Geometry of Accretion in Active Galactic Nuclei, In: Ho, L. (ed.), Carnegie Observatories Astrophysics Series, Vol. 1: Coevolution of Black Holes and Galaxies, Pasadena

Strateva, I.V., Strauss, M.A., Hao, L., Schlegel, D.J., Hall, P.B., et al., 2003, AJ, 126, 1720

Sudou, H., Iguchi, S., Murata, Y., \& Taniguchi, Y. 2003, Science, 300, 1263

Teerikorpi, P. 1997, ARA\&A, 35, 101

Terashima, Y., Iyomoto, N., Ho, L.C., \& Ptak, A.F. 2002 ,ApJS, 139 ,1

Thean A., Pedlar A., Kukula M.J., et al. 2001, MN 325, 737

Tran, H.D. 2001, ApJ, 554, 19 
Tran, H.D. 2003, ApJ, 583, 632

Trümper, J. 1983, AdSpR, 2, 241

Turner, T.J., \& Pounds, K.A. 1989, MNRAS, 240, 833

Turnshek, D.A., Bohlin, R.L., Williamson, R.L., et al. 1990, AJ, 99, 1243

Unsöld, A., Baschek, B., 1988, Der neue Kosmos, Springer Verlag Berlin

Urry, C.M., \& Padovani, P. 1995, PASP, 107, 803

Urry, C.M., Falomo, R., \& Scarpa, R. 1999, ApJ, 512, 88

van Bemmel, I., Barthel, P., 2001, A\&A, 379, L21

van den Broek, A.C., van Driel, W., de Jong, T., et al. 1991, A\&AS 91, 61

van Driel, W., de Jong, T., 1990, A\&A 227, 6

van Driel, W., van den Broek, A.C., de Jong T., 1991, A\&AS 90, 55

Veilleux, S., Zheng W., 1991, ApJ, 377, 89

Veilleux S., 2001, Spectroscopic Diagnostics for IR-Selected AGNs. In: Green, R.F., Khachikian, E.Y., Sanders, D.B. (eds.) AGN Surveys, Proceedings of IAU Colloquium 184, ASP

Véron-Cetty, M.-P., \& Véron, P. 2001, A catalogue of quasars and active nuclei (10th edition), Observatoire de Haute Provence

Véron-Cetty, M.-P., \& Véron, P. 2003, A\&A, in press

Véron-Cetty, M.-P., \& Véron, P. 2000, A\&ARv, 10, 81

Vestergaard, M. 2002, ApJ, 571, 733

Voges, W. 1993, AdSpR, 13, 391

Voges, W., Gruber, R., Haberl, F., Kuerster, M., Pietsch, W., Zimmermann, U., 1994, ROSAT NEWS No. 32

Voges, W., Aschenbach, B., Boller, T., et al. 1996, IAU Circ. 6420

Voges, W., Aschenbach, B., Boller, T, et al. 1999, A \& A 349, 389

Vollmer, B., Beckert, T., \& Duschl, W.J. 2004, A\&A in press 
Walter, R., Fink, H.H., 1993, A\&A 274, 105

Welsh, W.F., \& Horne K. 1991, ApJ 379, 586

White, R.L., Becker, R.H., Helfand, D.J., Gregg, M.D. 1997, ApJ, 475, 479

Wills, B.J., \& Browne, I.W.A. 1986, ApJ, 302, 56

Wills, B.J., \& Brotherton, M.S. 1995, ApJ, 448, L81

Wills, B.J. 1999, Introduction to Unified Schemes. In: Ferland, G., \& Baldwin, J. (eds.) Quasars and Cosmology, ASP Conference Series 162, p. 101

Wolstencroft, R.D., Savage, A., Clowes R.G., et al. 1986, MNRAS, 223, 279

Worral, D.M., Giommi, P., Tananbaum, H., Zamorani, G., ApJ, 313, 596

Worthey, G., 1994, ApJS 95, 107

Wright, A.E., Griffith, M.R., Burke, B.F., Ekers, R.D. 1994, ApJS, 91, 111

Wright, A.E., et al. 1996, ApJS 103, 145

Yi, I., Boughn, S.P., 1998, ApJ 499, 198

York, D.G., et al. 2000, AJ, 120, 1579

Young, P., \& Schneider, D.P. 1980, ApJ 238, 955

Zetzl, M., 2004, Diplomarbeit, Universität Göttingen

Zimmermann, H.-U., Boller, T., Döbereiner, S., Pietsch, W. 2001, A\&A, 378, 30 


\section{Danksagung}

Ich danke Herrn Prof. Dr. W. Kollatschny für die Betreuung dieser Arbeit, seine stete Hilfsbereitschaft sowie für die angenehme und motivierende Arbeitsatmosphäre.

Herrn Prof. Dr. S. Dreizler danke ich für die Übernahme des Korreferats.

Ich danke Herrn Prof. Dr. K. J. Fricke für die Ermöglichung dieser Arbeit an der Universitäts-Sternwarte.

Herrn Dr. W. Pietsch danke ich für die sehr angenehme und konstruktive Zusammenarbeit.

Ich danke Herrn Dr. M. Dietrich, Herrn P. Albrecht und Herrn Dr. D. Grupe dafür, dass sie mir Daten zur Verfügung gestellt haben.

Mein besonderer Dank gilt Herrn Dr. P. Papaderos und Herrn Dr. A. Goerdt für stete Hilfe und freundschaftliche Unterstützung.

Bei den Mitgliedern der Arbeitsgruppe bedanke ich mich für das angenehme Arbeitsklima.

Herrn A. Lehmann danke ich für die Korrektur des Manuskripts und den Beistand auf Tagungen in China.

Meinen Eltern danke ich für die Unterstützung meines gesamten Studiums.

Ich danke Frau Dr. M. Haller für die Korrektur des Manuskripts und für vieles andere.

Das dieser Veröffentlichung zugrundeliegende Projekt wurde mit Mitteln der Deutschen Agentur für Raumfahrtangelegenheiten (DARA) GmbH unter dem Förderkennzeichen 50 OR 94089 sowie der Deutschen Forschungsgemeinschaft (DFG) unter dem Förderkennzeichen Ko 857/24 gefördert. Die Verantwortlichkeit für den Inhalt dieser Veröffentlichung liegt beim Autor. 



\section{Lebenslauf}

Name: $\quad$ Karsten Bischoff

Geboren: $\quad$ 17.02.1966 in Hannover

Eltern: $\quad$ Gunda und Dr. Hermann Bischoff

Staatsangehörigkeit: deutsch

1972 - $1976 \quad$ Grundschule Windheim zu Döhren

1976 - $1985 \quad$ Städtisches Gymnasium Petershagen

21.05.1985 Abitur, Note ,sehr gut“

01.07.1985 - 30.09.1986 Grundwehrdienst

01.10.1986 - 05.02.1993 Studium der Physik, Georg-August-Universität Göttingen

27.10.1988 Diplomvorprüfung im Fachbereich Physik, Note „sehr gut“

1991 - 1992 Diplomarbeit zum Thema „Langzeitvariabilitätsanalyse morphologisch pekuliärer Seyfert-1 Galaxien“"

05.02.1993 Diplomhauptprüfung im Fachbereich Physik, Note ,sehr gut“

01.04.1993 - 28.02.1994 Wissenschaftliche Hilfskraft mit abgeschlossener Hochschulausbildung an der Georg-August-Universität Göttingen

01.03.1994 - 31.12.2003 Wissenschaftlicher Mitarbeiter an der Georg-August-Universität Göttingen

1998 - $2003 \quad$ Dissertation zum Thema „Multifrequenzanalyse eines Samples röntgen- und optisch selektierter Aktiver Galaktischer Kerne“ 


